O
O
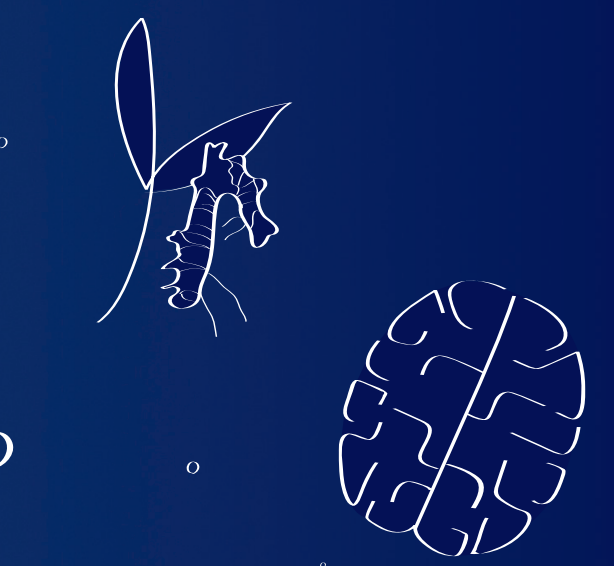

$\mathrm{O}$
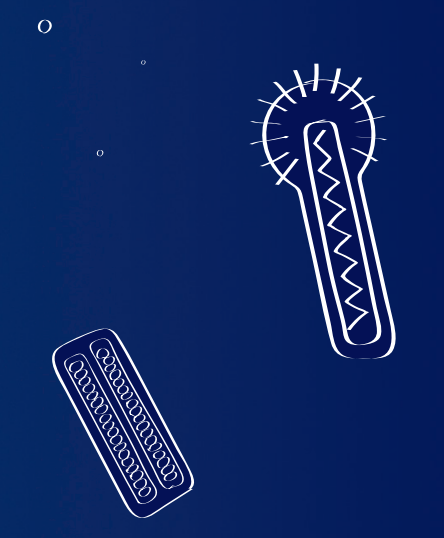

o

o

O

O

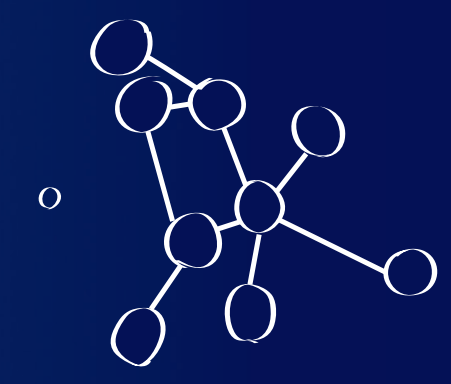

O

O

O

$\precsim$
$\frac{1}{1}$
$\frac{1}{9}$

o

O
Baculovirus-induced insect behaviour: from genes to brains

\section{Invitation}

You are cordially invited to attend the public defense of my PhD thesis entitled:

Baculovirus-induced insect behaviour: from genes to brains

On Friday, $31^{\text {th }}$ August at 16:00 p.m. in the Aula of

Wageningen University, Generaal Foulkesweg 1, Wageningen

Yue Han

hanyue0731@gmail.com

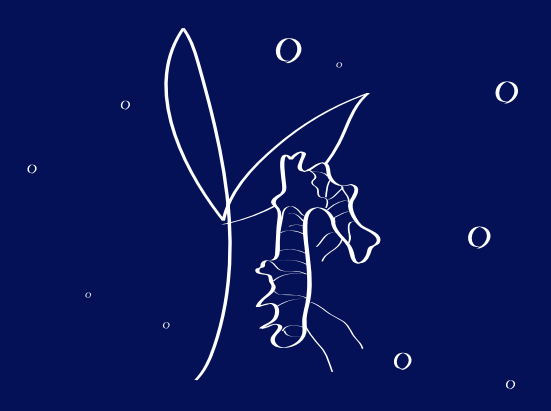

Paranymphs Yuxi Deng

Yuxi Deng

yuxi.deng@wur.n

corien.voorburg@wur.nl 
Baculovirus-induced insect behaviour:

from genes to brains

Yue Han 


\section{Thesis committee}

\section{Promotor}

Prof. Dr M.M. van Oers

Professor of Virology

Wageningen University \& Research

\section{Co-promotor}

Dr V.I.D. Ros

Assistant professor, Laboratory of Virology

Wageningen University \& Research

\section{Other members}

Prof. Dr L.E.M. Vet, Wageningen University \& Research

Prof. Dr J.A. Jehle, Julius Kühn Institute, Darmstadt, Germany

Prof. Dr A.T. Groot, University of Amsterdam

Dr R.P. van Rij, Radboud University Medical Center, Nijmegen

This research was conducted under the auspices of the Graduate School for Production Ecology and Resource Conservation. 


\title{
Baculovirus-induced insect behaviour: from genes to brains
}

\author{
Yue Han
}

\section{Thesis}

submitted in fulfilment of the requirements for the degree of doctor at Wageningen University by the authority of the Rector Magnificus

Prof. Dr A.P.J. Mol, in the presence of the

Thesis Committee appointed by the Academic Board to be defended in public on Friday August 31, 2018 at 4 p.m. in the Aula. 
Yue Han

Baculovirus-induced insect behaviour: from genes to brains, 190 pages.

$\mathrm{PhD}$ thesis, Wageningen University, Wageningen, the Netherlands (2018)

With references, with summary in English

ISBN: 978-94-6343-467-6

DOI: https://doi.org/10.18174/454937 


\section{Table of Contents}

Chapter 1 General introduction 1

$\begin{array}{lll}\text { Chapter } 2 \quad \text { Virus induced behavioural changes in insects } & 15\end{array}$

Chapter $3 \quad$ Parasitic manipulation of host behaviour: baculovirus SeMNPV 39

EGT facilitates tree-top disease in Spodoptera exigua larvae by extending the time to death

Chapter 4 Timely trigger of caterpillar zombie behaviour: temporal

requirments for light in baculovirus-induced tree-top disease

Chapter $5 \quad$ Baculovirus PTP2 functions as a pro-apoptotic protein

Chapter $6 \quad$ Substrate identification of baculovirus protein tyrosine phosphatase

Chapter 7 Baculovirus invasion of the lepidopteran central nervous system

Chapter $8 \quad$ General discussion

References

Summary

List of Publication

About the Author

Acknowledgements

Ceritficate 



\section{Chapter}

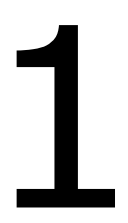

General introduction and thesis outline 


\section{Parasites and the extended phenotype}

The word 'parasite' is originally derived from the Greek term "parasitos", which was used to describe persons tasting food at the tables of noble families in order to check whether the food was poisoned or safe (Etymonline.com, 2017). Therefore, these "parasitos" were nourished without working and lived at the expenses of others. Later the use of this term was changed to describe organisms that live on the expense of others, and as such obtain their food from other animals or plants that are commonly called the host of the parasite (Long and Staskawicz, 1993; Mehlhorn, 2015). During billions of years of evolution, parasites and hosts have developed complex mutual relationships and it is known that parasites affect many aspects of their hosts, including metabolism, physiology, development, morphology, and behaviour (Tully and Nolan, 2002; van Houte et al., 2013). As compulsory intracellular parasites, viruses are also known to affect many aspects of their hosts (Mehlhorn, 2015; Poulin, 2000; van Houte et al., 2013).

In the famous book "The Selfish Gene" published in 1976, Richard Dawkins presented his "gene-centred view of evolution" theory. According to his theory, the basic unit of evolution is the individual gene instead of the individual organism or groups of similar organisms. Therefore, a gene is expected to maximize the number of copies of its own and to pass them on to the next generation (Dawkins, 1976). In the book "The Extended Phenotype" published in 1982, Richard Dawkins further expanded his evolutionary theory and introduced the concept "the extended phenotype". He proposed that the phenotype should not be limited to biological processes (such as protein synthesis, tissue growth), but should also include all the effects that a gene has on its environment. There are three forms of extended phenotypes: the first is the ability of animals to modify their environment using architectural constructions, such as dam-making behaviour of beavers. The second form is the ability to manipulate other organisms, also referred to as "parasitic manipulation". In this form, the observed host phenotype is a consequence of the expression of the parasite's genes. Famous examples include the suicidal behaviour of crickets infested by hairworms (Thomas et al., 2002) and the zombie behaviour of carpenter ants infected by fungi (Hughes et al., 2011). In this thesis, I will look into the behavioural and physiological phenotypes of caterpillars infected by baculoviruses (as further detailed below) and I will study how the expression of viral genes is affecting the observed host phenotypic behaviour. The third form of an extended phenotype includes the action at a distance of a parasite on its host, such as the behavioural manipulation by egg-laying female cuckoos, impelling other birds to feed the cuckoo chicks (Dawkins, 1982). 


\section{Baculoviruses}

The family Baculoviridae is a group of arthropod-specific viruses with double stranded, circular genomes ranging from 80 to $180 \mathrm{kbp}$ (van Oers and Vlak, 2007). Baculoviruses have been reported to infect more than 700 insect species belonging to the orders Lepidoptera, Diptera and Hymenoptera (Slack and Arif, 2007). Baculovirus genomes contain roughly 90 to 180 open reading frames (ORFs) (van Oers and Vlak, 2007). Two different types of virions, which are morphologically different but genetically identical, are produced during infection. Budded viruses (BVs) are responsible for systemic infection within the host and occlusion derived viruses (ODVs) are responsible for primary infection of the host's epithelial midgut cells. BVs consist of a single, enveloped nucleocapsid and obtain their envelope from the plasma membrane of infected cells. In contrast, ODVs may contain single or multiple nucleocapsids within a single envelope and ODVs obtain their envelope from the inner nuclear membrane of the infected cells. ODVs are embedded in a paracrystalline proteinaceous matrix, forming an occlusion body (OB). OBs are extremely stable and protect virions from harsh conditions (Slack and Arif, 2007). Based on the morphology of OBs, baculoviruses are named nucleopolyhedroviruses (NPVs) or granuloviruses (GVs) (Rohrmann, 2013b). NPVs and GVs differ in the protein that forms the paracrystalline matrix: polyhedrin for NPVs and granulin for GVs. According to the number of nucleocapsids within each ODV, NPVs can be further divided into multiple nucleopolyhedroviruses (MNPV) and single nucleopolyhedroviruses (SNPV). Taxonomically, baculoviruses are divided into four genera: Alphabaculovirus (lepidopteranspecific NPVs), Betabaculovirus (lepidopteran-specific GVs), Gammabaculovirus (hymenop teran-specific NPVs) and Deltabaculovirus (dipteran-specific NPVs) (Jehle et al., 2006). Based on phylogenetic analyses, the Alphabaculovirus genus is further divided into Group I and Group II NPVs (Zanotto et al., 1993). Group I NPVs use the GP64 protein as BV envelope fusion protein, whereas Group II NPVs and beta- and deltabaculoviruses use the F protein as the BV envelope fusion protein (Rohrmann, 2013b; Slack and Arif, 2007). Gammabaculoviruses do not have the BV phenotype and as a consequence are limited to the gut (Lucarotti et al., 2012).

The baculovirus infection cycle starts with ingestion of virus-contaminated food by insect hosts. OBs migrate together with food into the insect's midgut (Figure 1, Step A). Under the alkaline conditions ( $\mathrm{pH} 9-11)$ in the insect midgut, the OBs dissolve thereby releasing the occluded ODVs (Figure 1, Step A). Subsequently, ODVs travel through the peritrophic membrane, a network of proteins and chitin that protects the midgut epithelium (Hegedus et al., 2009). ODVs then bind and fuse with the microvilli of the midgut epithelium cells (more in particular the 
columnar cells) and start the first round of infection (Figure 1, step B) (Rohrmann, 2013a). After the first round of virus replication in the nucleus of the infected midgut cells BVs are produced (Figure 1, step B and C). These BVs then infect adjacent tissues or are transported to other tissues via the hemolymph and trachea, causing a systemic infection (Figure 1, step B and C) (Rohrmann, 2013a). Consequently, more BVs are produced from newly infected cells. In the late stage of infection, ODVs are produced in the nucleus of infected cells and packed into OBs (Figure 1, step C). After the insect has succumbed to the infection, viral enzymes such as chitinase and cathepsin (Rohrmann, 2013a) liquefy the cadaver, releasing OBs into the environment (Figure 1, Step D). OBs are produced in large numbers, and virus yields up to $10^{8}$ OBs per caterpillar have been reported for alphabaculoviruses (Rohrmann, 2013a).

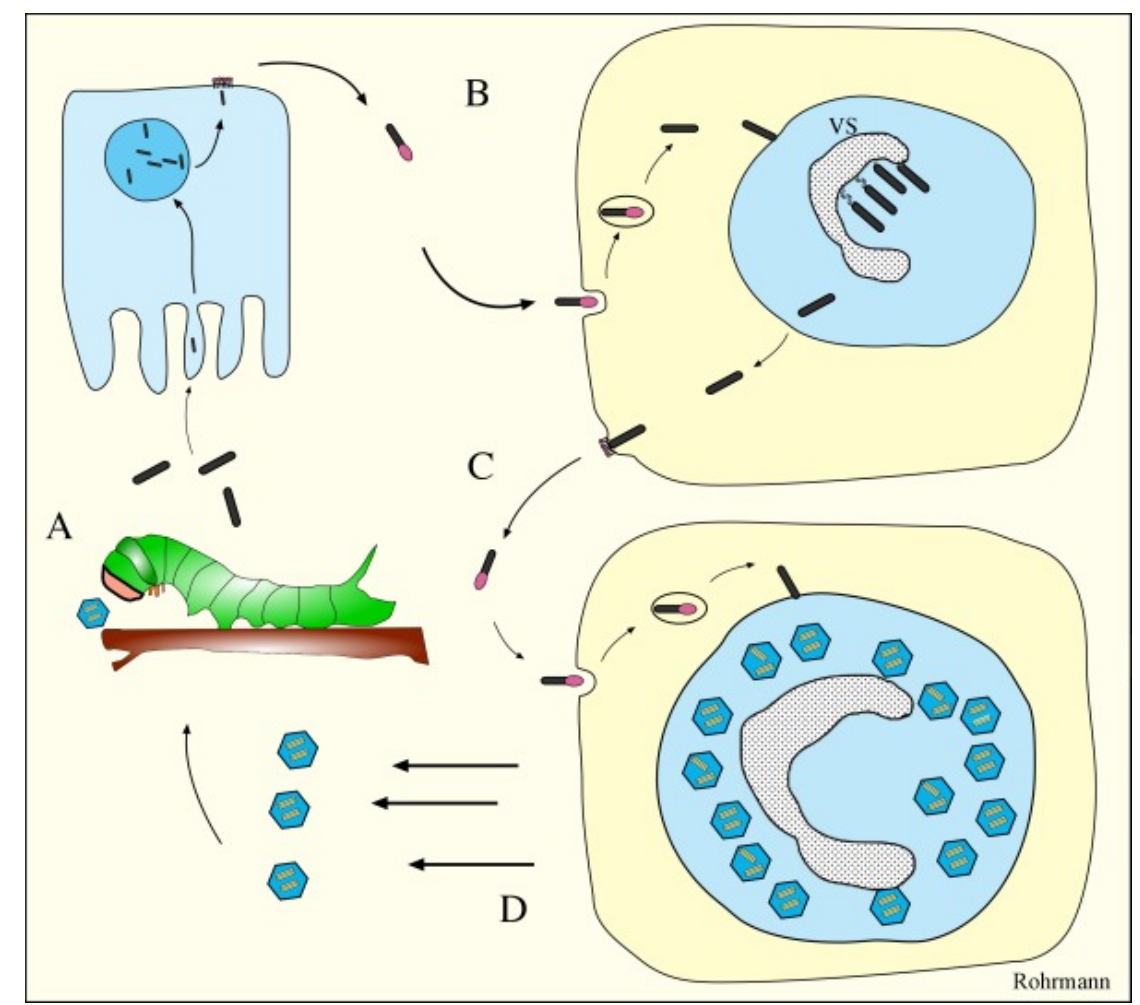

Figure 1. Baculovirus infection cycle (Rohrmann, 2013a) (see main text for explanation; figure used with permission from George Rohrmann).

Since several decades, baculoviruses have been used as biocontrol agents to control forest and agricultural pests, due to their efficacy, high specificity and safety for non-target organisms (Lacey et al., 2015; Moscardi, 1999). For example, Helicoverpa armigera nucleo polyhedrovirus (HearNPV) has been widely used in China to control the cotton bollworm 
H. armigera (Sun et al., 2006). Moreover, baculoviruses are widely used as vectors to produce recombinant proteins in insect cells, e.g. to produce subunit vaccines or virus-like particles [for recent reviews see Felberbaum (2015) and Liu et al. (2013)]. Recombinant baculoviruses are also being used to produce Adeno-associated virus (AAV) to be used as gene therapy vectors (Clément and Grieger, 2016; Urabe et al., 2002). Though baculoviruses cannot replicate in mammalian cells, they can enter the nucleus of many different mammalian cell types. Therefore, baculoviruses are also being used as vectors for gene delivery into mammalian cells (Condreay and Kost, 2007).

In the research presented in this thesis, viruses belonging to two different baculovirus species are used: Autographa californica multiple nucleopolyhedrovirus (AcMNPV) and Spodoptera exigua multiple nucleopolyhedrovirus (SeMNPV), both belonging to the genus Alphabaculovirus. AcMNPV is a generalist baculovirus that can infect more than 95 insect species from at least 15 families in the order Lepidoptera (Cory, 2003). On the contrary, SeMNPV is a specialist virus that only infects Spodoptera exigua (monospecific). SeMNPV can kill its host in a very short time with a low infective dosage [the $50 \%$ lethal time (LT 50 ; the time point at which $50 \%$ of a population dies for a given viral dose) is 3 to 3.5 days post infection in third instars infected with a $90-95 \%$ lethal concentration]. SeMNPV is widely used as a bio-pesticide to control S. exigua in the field and in greenhouses (Cai et al., 2010).

\section{The insect host - Spodoptera exigua}

Spodoptera exigua is used as host species in the experiments described throughout this thesis. Spodoptera exigua (Hübner) belongs to the family Noctuidea, order Lepidoptera. It is commonly known under the names beet armyworm, the small mottled willow moth, and the asparagus fern caterpillar. While S. exigua originates from Asia, it is now spread over tropical and subtropical areas throughout the world (Bianchi et al., 2000). This species does not tolerate cold winters, explaining the restriction to tropical and subtropical regions. However, it widely occurs in greenhouses all over the world. The insect was accidently introduced into the Netherlands in 1976 from Florida, USA (Smits and Vlak, 1988). Spodoptera exigua caterpillars are polyphagous and are a serious pest for ornamental and vegetable crops. The caterpillars feed on a variety of cultivated plants including cotton, tomato, celery, lettuce, cabbage and ornamental crops like chrysanthemum and gerbera (Cai et al., 2010), causing severe production losses each year. Spodoptera exigua caterpillars present army-like behaviour (hence, the name beet army worm): they will stay in one field patch until all the food supplies are consumed. 
After depletion of food, all the caterpillars move together like an army to seek for a new food source.

Under laboratory conditions, the generation time of $S$. exigua is ca. 20 days at $27^{\circ} \mathrm{C}$ (Elvira et al., 2010; Golikhajeh et al., 2017). Individuals go through five larval stages (named L1 to L5), a pupal stage and an adult stage. Adult female moths normally lay a cluster of 50-150 eggs on the back side of leaves in the field. At $27^{\circ} \mathrm{C}$ eggs hatch after 2-3 days and the developmental time for each of the larval stages is 2, 1.5, 1.5, 2, and 3-4 days, respectively (Azidah and SofianAzirun, 2006). First and second instars feed gregariously, while third, fourth and fifth instars feed solitarily (Azidah and Sofian-Azirun, 2006). Pupation happens in the soil. The actually developmental time of S. exigua in the field depends on many factors, including temperature, population density, food source and food abundance (Mehrkhou et al., 2012).

\section{Baculovirus-induced behavioural changes}

Baculoviruses affect their host in multiple ways, inducing behavioural manipulation and modulating host immune system (Clem, 2001, 2005; Kamita et al., 2005; Katsuma et al., 2012; van Houte et al., 2012). These changes potentially enhance virus transmission. The earliest known description of baculovirus-induced alteration of host behaviour dates back to 1891, when Hofmann described how diseased larvae of the nun moth Lymantria monacha climbed up and died in tree canopies (Hofmann, 1891). Later, this pre-death climbing behaviour was named "Wipfelkranheit" or "tree-top disease". It was only until several decades later that scientists found out that these larvae had died due to a baculovirus infection. Apart from treetop disease, baculoviruses also induce hyperactivity [also known as hypermobility or enhanced locomotor activity (ELA)] in their caterpillar hosts: infected caterpillars were found to move over larger distances than healthy individuals (Kamita et al., 2005; Katsuma et al., 2012; van Houte et al., 2012). Previous studies on baculovirus-induced behavioural changes led to the identification of two viral genes, which are involved in the observed behavioural manipulations. The ptp gene from the baculoviruses AcMNPV and Bombyx mori (Bm) NPV was reported to be involved in inducing hyperactivity in S. exigua and B. mori larvae, respectively (Kamita et al., 2005; Katsuma et al., 2012; van Houte et al., 2012). The egt gene from the baculovirus Lymantria dispar (Ld) MNPV was found to be responsible for tree-top disease in L. dispar larvae (Hoover et al., 2011), although a general role for egt in inducing tree-top disease is disputed (Ros et al., 2015). A detailed review of virus-induced changes in insect host behaviour, including baculovirus-induced behavioural changes in caterpillars, is presented in Chapter 2. 


\section{Modulation of the host immune system by baculoviruses}

Parasites often enhance their survival rate and transmission efficiency by modulating the host immune system (Gandon et al., 2009; Maizels and McSorley, 2016; Suderman et al., 2008). Some parasites actively induce apoptosis in specific host cell types to suppress the host immune system. Apoptosis is a process of programmed cell death that occurs in multicellular organisms and plays a crucial role in development and in removing damaged cells from the body. Features of apoptotic cells include cell shrinkage, DNA fragmentation, chromatin condensation, membrane blebbing and formation of apoptotic bodies (Elmore, 2007). There are two main pathways that can trigger apoptosis: the extrinsic or death receptor pathway and the intrinsic or mitochondrial pathway (Igney and Krammer, 2002). Caspases are the main molecules that induce apoptosis. They function as proteases that cut specifically at an aspartic acid residue of a target protein (Elmore, 2007). Both the extrinsic pathway and the intrinsic pathway lead to the same execution pathway that starts with the activation of caspase- 3 (the main effector caspase). Caspase-3 activates an endonuclease that degrades nuclear chromosomal DNA and causes chromatin condensation. Besides, caspase-3 induces cytoskeletal changes and cell lysis (Elmore, 2007). All these changes eventually lead to the formation of apoptotic bodies.

It is generally well appreciated that host-induced apoptosis is a common strategy of the host to eliminate virus-infected cells (Clem, 2005). By inducing apoptosis virus replication is terminated, thus reducing progeny virus production (Clem, 2005). In contrast, some viruses induce apoptosis in certain host cells to suppress the host immune system and/or enhance virus dissemination (Chitnis et al., 2011; Ni et al., 2017; Suderman et al., 2008). For example, the polydnavirus Microplitis demolitor bracovirus (MdBV) induced apoptosis in specific hemocytes of Spodoptera frugiperda caterpillars and this activity contributed to the immunosuppression of the host (Suderman et al., 2008). Baculoviruses contain pro-apoptotic genes, for example, the immediate early gene 1 (iel) from the baculovirus AcMNPV. Previous studies have shown that the expression of the iel gene from AcMNPV leads to the formation of apoptotic bodies in virus-infected Sf21 cells by initiating virus DNA replication events that subsequently trigger cell death (Schultz et al., 2009). Apart from pro-apoptotic genes (like iel), baculoviruses also contain anti-apoptotic genes, to suppress apoptosis. AcMNPV carries the anti-apoptotic gene $p 35$, while other baculoviruses contain either $p 35$ homologues or inhibitor of apoptosis (iap) genes (Clem, 2001). 


\section{The CNS in host behavioural manipulation}

While some details are known on how parasites modulate the host immune system, hardly anything is known about the mechanisms underlying parasitic behavioural manipulation. Parasites may achieve host behavioural manipulation via invading the host's central nervous system (CNS), the endocrine system, and/or the immunomodulatory system (Lafferty and Shaw, 2013). Neuromodulators are messenger molecules released from neurons in the CNS and are known to control a wide range of host behavioural traits. Several studies have shown that parasite-induced behavioural changes are accompanied by altered levels of neuromodulators (Adamo, 2002; Libersat et al., 2009; Ohkawara and Aonuma, 2016; Perrot-Minnot and Cezilly, 2013). For example, Manduca sexta parasitized by the parasitoid Cotesia congregata exhibited declined feeding and locomotion behaviour and this was accompanied by a sharp increase in octopamine content in the larval CNS (Adamo, 2002). Amphipods of the species Gammarus pulex showed a much stronger phototactic behaviour when infected with acanthocephalan parasites. In this example, the behavioural change was linked to an increase in the serotonin levels in the amphipod brains (Tain et al., 2006).

Baculoviruses may also alter host behaviour by affecting or invading the host CNS, and BmNPV and HearNPV have been detected in the brain of infected hosts (Herz et al., 2003; Katsuma et al., 2012; Torquato et al., 2006). The CNS of insect consists of a brain and a ventral nerve cord (Figure 2). The brain has two lobes and the ventral nerve cord consists of the subesophageal ganglion (SEG), three thoracic ganglia and seven abdominal ganglia. The brain and SEG are in the head region of the caterpillar and are connected by the paired circumesophageal connective (CirC). The brain and SEG form the higher-order control centre and regulate many different biological processes in the larvae (Ando and Kuwasawa, 2004; Cantera et al., 1995; Reichert and Boyan, 1997). However, in-depth knowledge on the larval lepidopteran brain is needed to further understand how baculoviruses might manipulate the insect brain. 


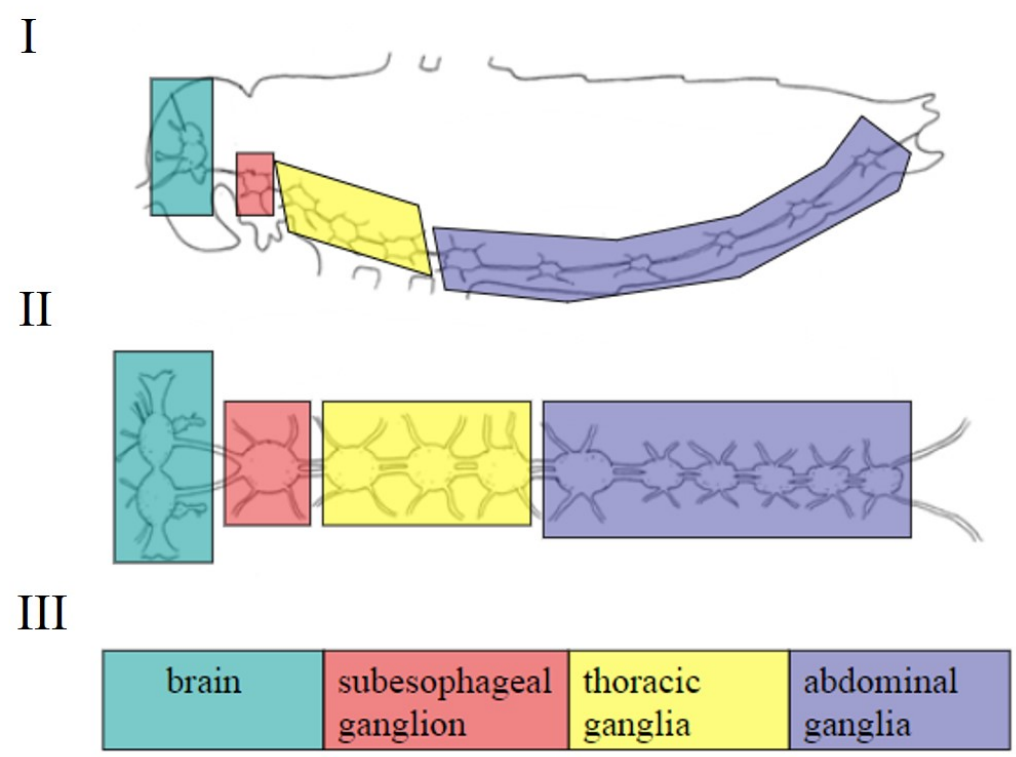

Figure 2. Schematic representation of the insect central nervous system (CNS). Panel I: the position of the CNS in the insect body (from left to right: head to tail). Panel II: the insect CNS consisting of the brain with two lobes, the subesophageal ganglion, three thoracic ganglia and seven abdominal ganglia. Panel III indicates the colour scheme used to indicate the different domains of the CNS (Picture from Clinton Nelson; http://slideplayer.com/slide/9069429/).

\section{Application of -omics to reveal mechanisms underlying parasitic manipulation}

To elucidate the molecular mechanism underlying manipulation of host behaviour by baculoviruses, previous studies on baculovirus-induced behavioural changes followed a candidate gene approach (Fitzpatrick et al., 2005; Hoover et al., 2011; Kamita et al., 2005; Katsuma et al., 2012; van Houte et al., 2012). However, this approach provides a relatively narrow view of the mechanisms behind behavioural manipulation. To discover novel genes for which a relation with regulation of behaviour was not known or expected before, methods that are able to monitor the expression of genes under various circumstances or the abundance of the encoded proteins need to be applied. The development of high-throughput methods like transcriptomics, proteomics and metabolomics enable such analyses, having the potential to generate global, unbiased and quantitative data. Such analyses benefit strongly from the availability of the genome sequence of the organism under study and in that aspect it is worthwhile mentioning here that the full genome of S. exigua has not been sequenced yet, only the genome of a closely related species [i.e. S. frugiperda (Gouin et al., 2017)] is known. 
Using a transcriptomic (or RNA sequencing; RNAseq) approach all RNA molecules produced in a tissue can be studied, including mRNAs, rRNAs, tRNAs, and other non-coding RNAs (Vijay et al., 2013). Studying the changes in the transcriptome as a response to different environmental conditions is currently one of the most accessible ways to reveal the molecular basis of phenotypic changes, including parasitic manipulation (Aubin-Horth and Renn, 2009). De Bekker et al. (2015) used an RNAseq approach to study the biting behaviour of carpenter ants induced by the fungus Ophiocordyceps unilateralis s.l. In that study, the transcriptomes of the carpenter ant during biting and after biting were compared. The results indicated that the fungus may regulate immune responses and neuronal stress in the host to induce the biting behaviour (de Bekker et al., 2015). In a follow-up study, the transcriptome of the parasite Ophiocordyceps kimflemingiae was determined under normal light-dark cycles and under continuous dark conditions. The results revealed that the fungal parasite might use biological clock genes to induce phase-specific activity in its host (de Bekker et al., 2017). Though transcriptomic approaches have been widely used in other studies, only a few studies have used transcriptomics to study parasitic manipulation.

With a proteomic approach, all proteins present in a tissue are studied simultaneously (Diz et al., 2012). Given the importance of proteins in signal transduction pathways, this method is of vital importance in studying parasitic manipulation. Pioneer studies on behavioural manipulation using proteomics were performed by Biron et al. (2005) to understand the water seeking behaviour of crickets parasitized by hairworms (Biron et al., 2005; Biron et al., 2006). These studies provided the first pieces of evidence that parasites induce behavioural changes via direct or indirect biochemical alterations and revealed that molecules secreted by the parasite could affect the development of the central nervous system (CNS) of the host. The molecules secreted by the hairworms were homologous to the Wnt family of proteins, known to play a role in signal transduction (Biron et al., 2006). To my knowledge, this is so far the only study investigating the molecular mechanisms of parasitic manipulation by using a proteomic approach.

\section{Outline of the thesis}

As described above, baculoviruses induce several behavioural and immunomodulation physiological changes in their host which eventually might enhance virus transmission. This thesis focuses on the possible mechanisms underlying these virus-induced modifications. 
In Chapter 2 the known examples of insect behavioural changes following virus infection are reviewed. Special attention is given to baculovirus-induced behavioural changes, with a focus on the role of several baculoviral genes in this process. Additionally, the ecological and evolutionary consequences of the virus-induced insect behavioural changes are discussed.

As outlined above, baculoviruses induce pre-death climbing behaviour (tree-top disease) in infected hosts. This process may involve viral and host genes, but environmental conditions may also affect the outcomes of behavioural manipulation. Hoover et al. (2011) showed that the egt gene from baculovirus LdMNPV was involved in inducing tree-top disease in L. dispar. Our previous work showed that SeMNPV induces light-dependent pre-death climbing behaviour in S. exigua (van Houte et al., 2014). The role of the egt gene in this process is investigated in Chapter 3. Besides host and viral genes, environmental conditions also affect the outcomes of behavioural manipulation. Since light plays a key role in triggering tree-top disease (van Houte et al., 2014), the importance of timing of light in this process was further analysed in Chapter 4.

Baculoviruses may modulate the immune system of their host and this may affect virus transmission. A protein tyrosine phosphatase (ptph2) gene from Microplitis demolitor bracovirus induces apoptosis in host immune system to enhance virus production. Chapter 5 aims to investigate the pro-apoptotic effect of SeMNPV protein tyrosine phosphatase PTP2 on cultured cells and on the immune system of $S$. exigua larvae and discusses how this might affect virus transmission.

In addition to tree-top disease, baculoviruses may also trigger hyperactivity in infected hosts. Parasites may achieve behavioural manipulation via invading or affecting the CNS of infected hosts. In many cases the expression of neurotransmitters is also altered. Therefore, it is hypothesized that AcMNPV induces hyperactivity by invading the $S$. exigua CNS and by changing the levels of neurotransmitters. In Chapter 6, immunocytochemistry is used to study the anatomy of the serotonin neurons in the third instar larval brain and SEG of $S$. exigua. A 3D model of the larvae brain is presented and it was studied whether AcMNPV infects the $S$. exigua larval brain and SEG.

The $p t p$ gene from AcMNPV as well as BmNPV is involved in the induction of hypermactivity in S. exigua and B. mori larvae, respectively (Kamita et al., 2005; Katsuma et al., 2012; van Houte et al., 2012). Moreover, it has been shown that the phosphatase activity of PTP is needed for the induction of hyperactivity by AcMNPV (van Houte et al., 2012). Viral and host proteins that interact with AcMNPV PTP are investigated in Chapter 7. This is achieved by purifying 
PTP from AcMNPV- infected S. exigua larvae and by subsequently identifying co-purified proteins using a proteomic analysis.

Finally, in Chapter 8 the results of the different chapters are discussed and placed in a broader perspective on parasitic manipulation. Furthermore, an outlook and some directions for future research are given. 


\section{Chapter}

\section{Virus-induced behavioural}

changes in insects

This chapter has been published as

Han Y, van Oers MM, van Houte S, Ros VID, 2015. Virus-induced behavioural changes in insects, in: Mehlhorn, H. (Ed.), Host Manipulations by Parasites and Viruses. Springer International Publishing, Cham, pp. 149-174. 


\begin{abstract}
Increasing evidence shows that host behaviour often changes following infection by a variety of parasites, including viruses. The altered behaviour is either induced by the parasites to enhance parasite survival and transmission, or is a response of the host to avoid spread of infection in the host population. Given the high prevalence of viruses among insects, in a virushost interaction or in a virus-vector relationship, viruses might have a huge impact on insect behaviour. This review first describes known examples of changes in insect behaviour upon virus infection. Although scarce, any known information on the underlying mechanism is also included. Special attention is given to baculoviruses and the hyperactivity and tree-top disease that they induce in their caterpillar hosts, so far the best studied systems in this research field. Subsequently, we discuss the virus-induced changes in insect behaviour from an ecological and evolutionary point of view.
\end{abstract}




\section{Introduction}

Insects are thought to be the most successful class among all known living organisms due to their high diversity and wide distribution: more than a million insect species have been described, which represent more than half of all known animal species, and insects can be found in almost all environments (Chapman 2006; Cranston 2010). Throughout their life cycle, insects encounter a variety of parasites, ranging from the smallest and simplest viruses to larger and more complex organisms like nematodes and parasitoids (van Houte et al. 2013; Cézilly et al. 2010). Insects are affected in many different ways upon infestation by parasites and this includes changes in development, metabolism, morphology, physiology and, most intriguingly, behaviour. The most well-known examples of host behavioural changes include the suicidal water-seeking behaviour of the cricket Nemobius sylvestris infested with the hairworm Paragordius tricuspidatus (Thomas et al. 2002) and the climbing behaviour of the carpenter ant Camponotus leonardi infected with the fungus Ophiocordyceps unilateralis (Hughes et al. 2011). Though many parasites have been reported to induce behavioural changes in insect hosts (Lefèvre et al. 2009; van Houte et al. 2013), the documentation on virus-induced changes in insect behaviour is scarce. Given the high prevalence of viruses among insects, either in a pathogenic interaction or in a virus-vector relationship (Miller and Ball 1998), viruses might have a huge impact on insect behaviour. In this review, we give an overview of the documented changes of insect behaviour upon infection by viruses and reveal what is known about the underlying molecular mechanisms.

Host behavioural changes upon infection by a parasite can be adaptive to the parasite or to the host. Many cases that clearly show enhancement of parasite transmission have been treated as compelling examples of the concept of the "extended phenotype" (Thomas et al. 2005; van Houte et al. 2013). The concept was proposed by Dawkins in 1982, and is defined as "genes from one organism (the parasite) having phenotypic effects on another organism (the host)" (Dawkins 1982). However, the behavioural changes are not always advantageous for the parasites, but can also be adaptive to the host to reduce the pathogenic effects of the infection to the individual or the population, or just be pathological side effects (Thomas et al. 2005; van Houte et al. 2013).

The first part of this review deals with the known examples of insect behavioural changes following infection by viruses that use insects as hosts or vectors, and, if known, the underlying mechanisms of such behavioural changes are explained. An overview of these virus-induced 
behavioural changes is given in Table 10.1. Special attention is given to baculovirus-induced behavioural changes in caterpillars. Because extensive information is available on baculovirus genomics and virus mutants can be easily made, several viral genes behind baculovirus-induced behavioural changes have successfully been identified. In the second part of this review we discuss the ecological and evolutionary consequences of virus-induced insect behavioural changes.

\section{Leptopilina boulardi Filamentous Virus (LbFV) Induces Superparasitism in the} Parasitoid Wasp L. boulardi

Leptopilina boulardi is a solitary parasitoid (order Hymenoptera) that usually lays a single egg per Drosophila host larva (order Diptera) and rejects already parasitized larvae, since only one wasp larva can successfully develop within the body of a Drosophila larva. Upon superparasitism (when a wasp parasitizes a Drosophila larva that has already been parasitized by another wasp), within-host larval competition occurs and only one of the wasp larvae survives. Usually, egg-laying wasp females avoid superparasitism, although it might occur under specific circumstances when fly hosts are rare (van Alphen and Visser 1990). L. boulardi filamentous virus (LbFV, an unclassified double-stranded (ds) DNA virus) infects $L$. boulardi wasps and increases the tendency of $L$. boulardi females to accept already parasitized fly hosts, which allows horizontal transmission of the virus from infected to uninfected L. boulardi larvae within the superparasitized Drosophila host (Patot et al. 2009; Varaldi et al. 2003), Theoretical models predict that such superparasitism behaviour is beneficial to the virus (Gandon et al. 2006), since the virus can invade new wasp lineages. It is hypothesized that the virus is injected together with the wasp egg into the Drosophila host during oviposition and that it infects the emerging parasitoid during its larval development (during which the parasitoid consumes the virus-infected Drosophila hemocoel). If a single parasitoid egg is present, vertical transmission occurs, because the parasitoid larva picks up the virus that was injected by its mother into the hemocoel of the Drosophila host. If two or more wasp eggs are present in one Drosophila larva, horizontal transmission may occur (when the progeny from an uninfected wasp present in the Drosophila larva picks up the virus delivered during subsequent superparasitsm by a virus-infected wasp female). The efficiency of horizontal transfer depends on the time between successive ovipositions (Varaldi et al.2009), which determines the difference in age and hence competition ability of the already present and incoming larvae. $L$. 
boulardi females pierce host larvae with their ovipositor to detect chemical cues associated with previous infestations. Although the exact mechanism behind the LbFV-induced superparasitism is unknown, it is hypothesized that LbFV affects chemoreceptor neurons in the ovipositors used to detect chemical cues associated with previous infestations, either through cell lysis or modulation of gene expression (Varaldi et al. 2009).

The influence of LbFV on other behavioural traits of the parasitoid wasps has also been investigated (Varaldi et al. 2006, 2009). The virus did not affect the circadian rhythms of the wasps, nor the ability of infected males to locate females through detection of sex pheromones, the ability of females to locate odours of fly larvae, the search patterns of foraging females, or the ability of females to discriminate between good and poor quality fly larvae. On the other hand, viral infection lead to a strong reduction ( $45 \%$ ) of locomotor activity of infected wasp females (while no effect was detected in males), possibly because of resource-allocation to wasp traits that benefit virus transmission, like egg-load, which was increased by $11 \%$ for infected females (Varaldi et al. 2005). This increase in egg-load might compensate for the higher risk of an infected female being egg-limited (due to superparasitism; single wasp females have been found to lay more than 9 eggs in a single Drosophila host). The increase in egg-load might be an adaptive response of the parasitoid to the presence of the virus, or a component of the virusinduced manipulation (Gandon et al. 2006). LbFV also affects the immune response of the Drosophila larvae against the parasitoids (Martinez et al. 2012). Virus-infected parasitoids are less frequently encapsulated (the main defense mechanism against parasitoids) than uninfected ones. This is not just a consequence of increased rates of superparasitism, but is also found in monoparasitized larvae.

\section{Iridovirus Infection Changes Sexual Behaviour in Crickets and Beetles}

Activation of the immune system has been shown to reduce sexual activity in a variety of animals, including invertebrates (Adamo 2008; Lawniczak et al. 2007). In general, induction of immune responses in the field cricket Gryllus campestris, the ground cricket Allonemobius socius and the sagebrush cricket Cyphoderris strepitans (order Orthoptera) reduces the calling frequency of males, thereby limiting their chances to mate with females (Fedorka and Mousseau 2007; Jacot et al. 2004; Leman et al. 2009). Reduced mating rates and other sickness behaviour that signal danger to healthy individuals are beneficial for the host population in which a sick animal lives, but would negatively affect sexually transmitted parasites that rely 
on mating for their dispersal (Adamo 2014). It is therefore evident that sexually transmitted viruses should have developed strategies to counteract host immune responses that lead to reduced sexual activity in order to safeguard their transmission.

An example is seen in an iridovirus isolated from crickets (CrIV, a strain of invertebrate iridescent virus 6 (IIV-6), family Iridoviridae, genus Iridovirus) (Kleespies et al. 1999; Jakob et al. 2002), which is sexually transmitted between Gryllus texensis crickets. Induction of immune responses normally leads to sickness behaviour, including a reduction in male calling rates, but this was not the case upon IIV-6/CrIV infection (Adamo et al. 2014). CrIV infection did not induce other sickness behaviours either, such as illness-related anorexia (Adamo et al. 2010). When CrIV-infected crickets were immune-challenged with heat-inactivated bacteria, they took longer to start courtship singing, in contrast to uninfected crickets that showed a shorter latency to court upon immune-challenge. A likely explanation for these observations is that CrIV infects the major components of the insect's immune system. The virus infects hemocytes and massive virus replication in the fat body has been reported (Kleespies et al. 1999). As a consequence of fat body infection, the level of immune-related host proteins strongly declined (Adamo 2014; Adamo et al. 2014). The hypothesis is that due to the reduced level of circulating immunomodulators the communication between the immune system and nervous system is hampered, preventing the induction of sickness behaviour. Since the immune system itself is attacked by CrIV, the crickets can no longer respond to bacterial stimuli either.

In contrast, Phyllophaga vandinei beetles (order Coleoptera) infected with the IIV-6 strain Chilo iridescent virus (IIV-6/CIV) showed strongly reduced mating behaviour (Jenkins et al. 2011). Pathologically not much is known of this virus/host combination, but it may be that infection is not efficient enough to completely overtake the fat body in this putatively nonnatural virus/host combination. On the other hand the CIV strain is known to be transmitted efficiently via contaminated food (Nalçacioğlu et al. 2009) and, therefore, it may not be advantageous for virus transmission to keep host mating levels high.

\section{Helicoverpa zea Nudivirus 2 Alters the Mating Behaviour of the Corn Earworm} Moth H. zea

Helicoverpa zea nudivirus 2 [HzNV-2, formerly known as Hz-2 $\mathrm{V}$ and as gonad-specific virus (GSV); family Nudiviridae] manipulates the physiology and behaviour of its host, the corn earworm moth $H$. zea (order Lepidoptera), in such a way that viral transmission is enhanced 
(Burand 2009; Burand et al. 2005). HzNV-2 replicates exclusively in the reproductive tissues of both male and female moths and this leads to malformation of these tissues and sterility of the moths (Raina and Adams 1995; Burand et al. 2005). In addition, female moths can be fertile, asymptomatic carriers that can transmit the virus vertically to their offspring (Hamm et al. 1996).

H. zea females may mate several times, but generally mate once during a given night (Burand 2009; Raina et al. 1994). This mating pattern is regulated by the sex pheromone level of the adult female. Pheromone production peaks during the second and third night after adult emergence and triggers female mating and calling behaviour, which attracts males. After mating, pheromone levels decline, and transfer of male-derived anti-calling factors that are part of the seminal fluid result in the loss of female sexual receptivity (Burand et al. 2005). Virus-infected females, however, continue calling after contact with males. This is a consequence of the presence of a 'virus plug' covering the reproductive opening of the female moths, preventing the transfer of anti-calling factors. Moreover, it was shown that infected females attracted twice as many males as uninfected females, which was a result of increased pheromone levels. However, males could not effectively mate with infected females due to presence of the virus plug. Nevertheless, during the short contacts (mating attempts) males could be contaminated with the virus, which they could transfer to other (uninfected) females during subsequent matings (Burand et al. 2004).

While uninfected males were more attracted to infected females than to uninfected females, HzNV-2 infected males had no preference for infected over uninfected females, and in fact responded less quickly to female calls. Most mating attempts of infected males were unsuccessful, although sexual contacts did occur, long enough for the virus to be transmitted. Infected males lacked accessory glands and most likely do not produce anti-calling factors. Therefore, females continued calling after mating attempts with infected males. Subsequent mating of these females with healthy males resulted in infected offspring (either asymptomatic or sterile progeny) being produced (Burand and Tan 2006).

\section{Hygienic Measures of Honeybees Against Virus Infections}

Kulinčević and co-authors reported in 1969 that honeybees (order Hymenoptera) that were infected with an as yet unidentified virus, causing the bees to become black and hairless, were attacked by conspecifics and were occasionally stung to death (Kulinčević et al. 1969). It is not 
exactly clear which virus is responsible, but this phenomenon reflects the ability of social insects to recognise infected nest mates. Social insects, such as honeybees (Apis mellifera) live together in dense populations with a high frequency of physical contact and a high degree of genetic homogeny. To limit the risks of disease transmission, social insects display various forms of antiseptic behaviour, reviewed by Wilson-Rich et al. (2009). Worker bees may limit damage by early removal of diseased brood from the colony, a phenomenon described as hygienic behaviour. Hygienic behaviour may occur in addition to undertaking (the removal of dead adults from the hive) and grooming (Wilson-Rich et al. 2009).

Bee virus infections often occur together with brood parasitism by Varroa destructor mites (Ball 1989). These mites parasitize bee nymphs, increase virus susceptibility of bees (Yang and Cox-Foster 2005) and serve as vectors for virus transmission, e.g. Gisder et al. (2009). The combination of Varroa mites and bee viruses poses a severe threat to bee colonies. A theoretical study showed that beehives that can reduce Varroa infestation by grooming and hygienic behaviour could in theory limit virus prevalence (Sumpter and Martin 2004). In practice, worker bees are able to recognise Varroa mite infestation and remove infested brood. Brood infested by mites with high virus titres were more efficiently removed, as was shown for deformed wing virus (DWV, family Iflaviridae, genus Iflavirus) (Schöning et al. 2012).

Bee colonies seem to vary considerably in the ability to recognise Varroa infested brood (Harris 2007). Changes in cuticular hydrocarbon profiles in bee pupae and emerging bee adults may be responsible for the recognition of Varroa-infested brood and parasitized emerging adults (Salvy et al. 2001). Changes in hydrocarbon composition were also reported for DWVinfected adult bees and these bees had a much higher chance of being bitten and removed from the hive than non-infected nest mates (Baracchi et al. 2012). Cuticular hydrocarbon profile changes were also found after immune stimulation with bacteria (Richard et al. 2008, 2012). The altered hydrocarbon composition seems to be a general immune response that may signal healthy workers to avoid or actively remove infected nest mates, allowing counter action against disease at a population level.

\section{Bee Viruses and Changes in Honeybee Neuronal Physiology}

Viruses that infect honeybees may affect sensory perception and learning ability. Infection with DWV alters the responsiveness to sensory information, leading to proboscis extension at lower sucrose concentrations than seen for non-infected bees (Iqbal and Mueller 2007). In addition, 
associative learning and memory formation were disturbed in DWV-infected bees. When an olfactory signal was given before a rewarding sucrose stimulus, infected animals did not learn to respond to the olfactory signal (Iqbal and Mueller 2007). This could be caused by replication of DWV in the brains, especially in regions associated with vision and olfaction (Shah et al. 2009). Brain infection may also have led to the aggressive bees observed in Japan that were infected with a genetic variant of DWV, Kakugo virus (Fujiyuki et al. 2004, 2005), but DWV itself has not been associated with aggressiveness (Rortais et al. 2006). Honeybees infected with Israeli acute paralysis virus (IAPV, family Dicistroviridae), also showed a lower sucrose threshold (Li et al. 2013). IAPV significantly reduced the ability of pollen foragers to return to the hive, in line with the fact that IAPV diseased bees are often found outside the hives. These changes in behaviour were accompanied by high IAPV titers in the heads, leading to the hypothesis that IAPV infection interfered with brain functions responsible for learning and navigation ( $\mathrm{Li}$ et al. 2013). A possible advantage for the bee population is that this virusinduced mal-orientation may protect against further virus-spread within the hive.

\section{The Parasitoid Dinocampus coccinellae Uses an Iflavirus to Change the Behaviour of the Ladybeetle Coleomegilla macaluta}

A unique example of parasitic manipulation is seen for Dinocampus coccinellae (order Hymenoptera), a parasitic wasp that lays its eggs in the ladybeetle Coleomegilla maculate(order Coleoptera) and uses a virus as a 'biological weapon' to manipulate the behaviour of the ladybeetle (Dheilly et al. 2015). The wasp larvae develop within the body of the ladybeetle and after circa 20 days a single prepupa egresses and spins a cocoon between the ladybeetle's legs. The ladybeetle serves as a bodyguard to protect the parasitoid cocoon from predation by covering the cocoon with its body (Dheilly et al. 2015; Maure et al. 2013). The behaviour of the ladybeetle is altered during this process; it is partially paralysed and displays tremors, suggesting a neurological disorder. A new virus was discovered, D. coccinellae paralysis virus (DcPV) (family Iflaviridae), which replicates mainly in D. coccinellae larvae, and is highly abundant in the oviduct cells of adult wasps. Before egression, the parasitoid larva transmits DcPV to the ladybeetle. The virus then replicates in the cerebral ganglia of the ladybeetles and induces a severe neuropathy, resulting in paralysis and tremors. Even so, an antiviral immune response is induced in the ladybeetle, which eventually leads to elimination of the virus, and recovery of normal behaviour. A range of parasitoids induce bodyguarding behaviour in their 
insect hosts (Maure et al. 2013; van Houte et al. 2013), and while the mechanisms have rarely been explored, other parasitoids may use viruses to induce bodyguarding behaviour.

\section{Baculovirus-Induced Behavioural Changes in Caterpillars}

The earliest known description of behavioural alterations in diseased insects dates from 1891, likely representing the oldest written account of behavioural manipulation by parasites in history. In this work Hofmann illustrates how larvae of the nun moth Lymantria monacha (order Lepidoptera) climb up and die in tree canopies. These larvae succumbed to an unknown agent, which made them crawl out of their common diurnal hiding place to die on exposed parts of the trees. This phenomenon was named Wipfelkrankheit, more commonly known as tree-top disease (Hofmann 1891). It was discovered several decades later that these larvae had died due to an infection with a baculovirus, causing behavioural alterations in the infected caterpillars and liquefying them to efficiently spread progeny viruses into the environment. Subsequent studies reported higher dispersal rates and aberrant climbing behaviour of infected caterpillars compared with healthy ones, leading to death at elevated positions (Smirnoff 1965; Evans and Allaway 1983). The first quantitative studies on baculovirus-induced changes of host behaviour were performed using Mamestra brassicae larvae infected with the baculovirus $M$. brassicae multiple nucleopolyhedrovirus (MbMNPV) (Vasconcelos et al. 1996; Goulson 1997). Infected larvae were found to move 3-5 times further within a given time than uninfected ones, and the majority of larvae died on the apical, more exposed part of plant leaves.

The behavioural repertoire induced by baculoviruses (family Baculoviridae) in their caterpillar hosts (order Lepidoptera) includes both tree-top disease (pre-death climbing behaviour) and hyperactivity (wandering over larger areas). For a long time, nothing was known about the proximate mechanisms that might govern these behavioural changes. In 2005, Kamita and coauthors identified the first 'behavioural' gene in a baculovirus by demonstrating the involvement of the protein tyrosine phosphatase (ptp) gene from Bombyx mori (Bm) NPV in hyperactive behaviour in larvae of the silkworm B. mori. Similarly, van Houte et al. (2012) showed that the ptp gene of Autographa californica (Ac) MNPV induced hyperactive behaviour in its host Spodoptera exigua. It was hypothesized that the viral ptp gene plays a conserved role in the induction of hyperactivity in a subset of baculoviruses (a monophyletic clade in the genus Alphabaculovirus, named group I) (van Houte et al. 2012). 
A study on Lymantria dispar (Ld) MNPV showed that the viral ecdysteroid-UDP-glucosyl transferase (egt) gene is required for death at elevated positions (tree-top disease) of gypsy moth (L. dispar) larvae (Hoover et al. 2011). However, the role of egt as a 'gene for an extended phenotype' (Hoover et al. 2011) seems not generally applicable: in tree-top disease induced by the baculovirus AcMNPV in both $S$. exigua and Trichoplusia ni caterpillars the viral egt gene does not play a role (Ros et al. 2015). When the egt gene was deleted from the viral genome, infected larvae died at the same height as wild type-infected larvae. Earlier studies on egt had shown that the encoded enzyme suppresses larval moulting, leading to an extended time to death. In certain caterpillar species, moulting is preceded by up- or downward movement of larvae; therefore, in some virus-host combinations, egt might have an effect on tree-top disease through an effect on larval moulting (Ros et al. 2015). In AcMNPV-infected S. exigua and $T$. $n i$ the egt gene did affect moulting-related climbing behaviour. It did however not affect treetop disease, which, in these virus-host combinations, occurred at a later stage of the infection than the moulting-related climbing. The apparent effect of egt on tree-top disease is dependent on the specific virus-host interaction and likely is influenced by factors such as the viral dose, time of infection (e.g. compared to the time of moulting), time of death and the intrinsic behaviour of the host. Since the role of egt in tree-top disease is not universal (Ros et al. 2015), other viral genes are likely to be involved.

While AcMNPV infection induces tree-top disease in the two lepidopteran hosts $T . n i$ and $S$. exigua, the phenotype appears to be host-dependent (Ros et al. 2015). In T. ni infected as 3rd instars, virus infection always caused the larvae to climb up and die at elevated positions, regardless of the larval stage at death. For $S$. exigua however, only larvae that had moulted during the infection (from 3rd to 4th instar) climbed up and died at elevated positions. Those that did not undergo moulting moved downwards and died at low positions. Additional experiments showed that this moulting-dependent climbing behaviour was also seen for $2 \mathrm{nd}$ instar larvae, and hence may be independent of the stage of the larvae at infection. When 2nd instar larvae were infected, only larvae that moulted during the infection (from 2nd to 3rd instar) died at elevated positions $(80.0 \mathrm{~mm} \pm 9.5)$, while those that did not moult (died as 2nd instar) moved downwards and died at low positions $(0.0 \mathrm{~mm} \pm 0.0)$ (Figure 10.1). These moultingdependent differences in pre-death climbing behaviour might be related to the normal (moulting-related) climbing behaviour that these two species display in the absence of virus infection. Downward movement was also observed for larvae of the winter moth Operopthera brumata infected with $O$. brumata NPV (OpbuNPV) (Raymond et al. 2005). Infected larvae 
descended from the foliage to the lower tree stems to die there, which might contribute to virus persistence and transmission. OpbuNPV occlusion bodies persist better on stems than on foliage and virus transmission differs in this system, since $O$. brumata is a forest dwelling species.

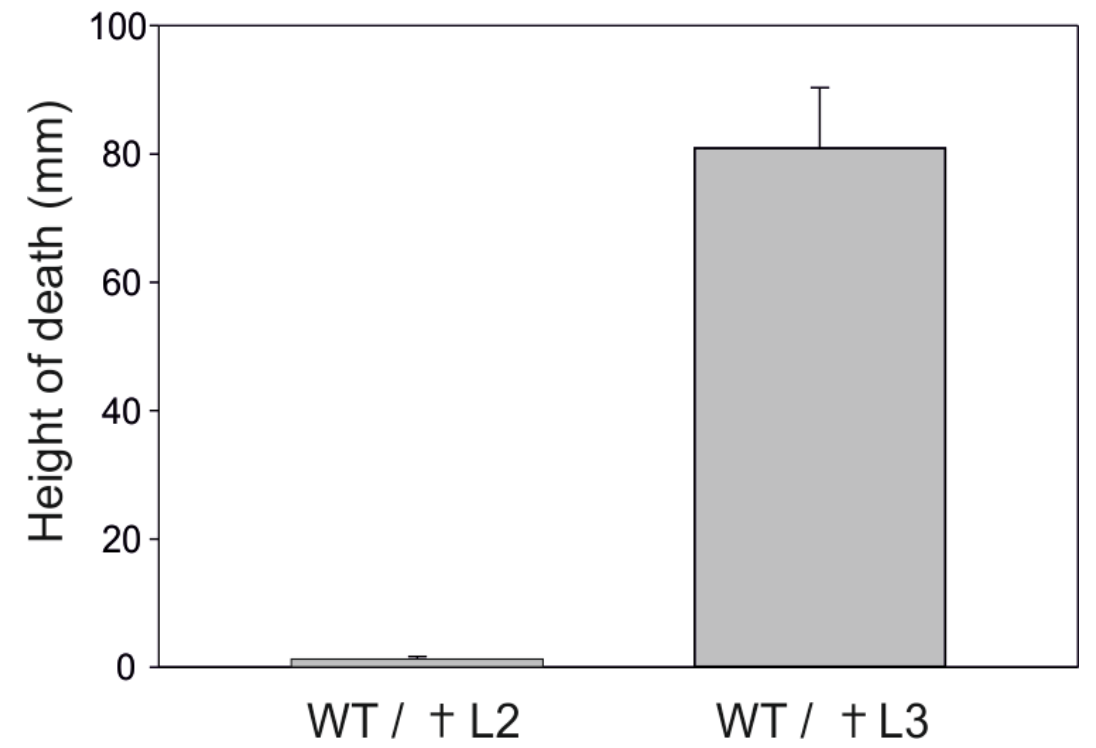

Figure 1. Average height at death (mm) of wild type (WT) AcMNPV-infected larvae (infected as second instars) that died as either second ( $\dagger \mathrm{L} 2 ; \mathrm{n}=6)$ or third ( $\dagger \mathrm{L} 3 ; \mathrm{n}=10)$ instars. Error bars indicate standard error of the mean. [Methods: S. exigua late 1st instar larvae were starved overnight. The next morning, newly moulted 2 nd instar larvae were infected by droplet feeding using a viral dose of $10^{\wedge} 7 \mathrm{OBs} / \mathrm{ml}$ of WT AcMNPV as described previously (van Houte et al. 2012). Larvae were placed individually in glass jars (van Houte et al. 2014a) to allow climbing and their final position of death was monitored when all larvae were dead (see Figure 10.2b for experimental set-up)].

Another important factor involved in the induction of tree-top disease is light (van Houte et al. 2014b). The specialist baculovirus $S$. exigua (Se) MNPV induced tree-top disease in S. exigua larvae, and prior to death, larvae were strongly attracted to light. When light was presented from above, infected larvae died at elevated positions, however, when light was presented from below, or no light was present at all, larvae died at low positions. Since climbing of uninfected larvae was light-independent (pre-moult climbing behaviour occurred also under completely dark conditions), the light-dependent climbing seen in tree-top disease was specifically evoked by virus infection (van Houte et al. 2014b). Possibly, baculoviruses hijack existing host pathways, that are for instance required for moulting-related climbing behaviour (through egt), or pathways related to light perception or phototaxis. However, baculoviruses do not use light as a uniform mechanism to trigger tree-top disease in caterpillars. Tree-top disease induced by LdMNPV in L. dispar larvae (K. Hoover, personal communication) or by AcMNPV 
in $T . n i$ larvae (V.I.D. Ros, unpublished data) was light-independent, since it was also observed in dark conditions. This indicates that baculoviruses may use different mechanisms to trigger tree-top disease.

While hyperactivity and tree-top disease are both induced by baculoviruses in caterpillar hosts, and can both be induced in a single infection, they seem to be caused by two independent manipulative mechanisms (van Houte et al. 2014a). The AcMNPV ptp gene was shown to induce hyperactivity, but not tree-top disease in its S. exigua host (van Houte et al. 2014a). Another indication that tree-top disease and hyperactivity are governed by different mechanisms is that these two phenotypes are triggered at different time points: hyperactivity is induced at 3 days post infection (around $70 \mathrm{~h}$ post infection) (van Houte et al. 2012), and the climbing behaviour leading to tree-top disease starts from 75 hpi onwards (Ros et al. 2015).

In conclusion, baculovirus-induced manipulation of caterpillar behaviour seems the result of a complex interplay between virus and host. Different behavioural displays are induced (hyperactivity and tree-top disease), by different causative mechanisms (see above). The expression of the different phenotypes is dependent on the virus-host system studied. Although the role of the viral $p t p$ in hyperactivity seems to be conserved within group I NPVs, this gene is absent in group II NPVs and in granuloviruses (genus Betabaculovirus). Viruses in these latter two groups have also been shown to induce hyperactive behaviour in lepidopteran hosts [e.g. MbMNPV in M. brassicae larvae, (Goulson 1997)], which implies that different viral genes might be involved in inducing hyperactivity. Which virus and/or host genes, proteins or pathways act downstream of the viral ptp and egt gene need to be determined. A genome-wide approach (transcriptomics/proteomics) can aid in selecting candidate genes or proteins involved in behavioural manipulation (van Houte et al. 2013).

\section{Dicistroviruses Affect Aphid Behaviour}

Aphids are hosts to a wide variety of microorganisms, including viruses, bacteria and fungi. All these microorganisms potentially have a major impact on aphid ecology (Ban et al. 2008). Two viruses have been reported to change the behaviour of aphids. Aphid lethal paralysis virus (ALPV, family Dicistroviridae) induced an unusual movement syndrome in its aphid host Rhopalosiphum padi (order Hemiptera): infected aphids moved away from their food source and became uncoordinated (Williamson et al. 1988). Whether this behavioural change is beneficial to the virus or to the host is not known. Rhopalosiphum padi virus (RhPV, 
family Dicistroviridae) is closely related to ALPV and was found to change the aggregation behaviour of aphids. Normally, R. padi aphids aggregate and are attracted to the odour of conspecific aphids. However, when $R$. padi aphids were infected with RhPV, they were not attracted to the odour of uninfected aphids any longer. Moreover, both infected and uninfected aphids were not attracted to infected aphids (Ban et al. 2008). The perception of repellent chemicals also changed after virus infection: infected aphids did not respond to methyl salicylate, an indicator of host suitability, and became more sensitive to the aphid alarm pheromone (Ban et al. 2008). It might be that these behavioural changes are beneficial to the host population, aimed at reducing the risk of infection for other aphids (usually of the same clone) sharing the host plant, and/or are beneficial to the virus since the virus is spread over a larger area, where aphids of other clones are more likely to be encountered.

\section{Plant Viruses Change the Behaviour of Aphid, Thrips and Whitefly Vectors}

Insects may function as vectors for plant viruses. Since the hosts for plant viruses are sessile, the potential for behavioural manipulation is restricted to the vectors, although viruses may affect the volatile profiles secreted by plants (Medina-Ortega et al. 2009). Several plant viruses have been shown to increase their transmission by affecting the feeding behaviour of the insect vector, changing food choices and altering the probing period and frequency (Ingwell et al. 2012; Fereres et al. 1990; Hu et al. 2013). The type of transmission (persistent versus nonpersistent) appears to have a direct correlation with the feeding behaviour observed for plant virus vectors (see review by Mauck et al. 2012). Non-viruliferous vectors show a strong attraction to infected plants, both for plants infected with persistently and non-persistently transmitted viruses. Persistently transmitted plant viruses such as potato leaf roll virus (PLRV, Luteoviridae) induce longer feeding times in aphid vectors needed to guarantee sufficient uptake of the virus from the phloem. For non-persistently transmitted viruses such as Potato virus Y (PVY, Potyviridae) aphids generally leave more quickly from infected plants than from healthy plants and the aphids can immediately transmit the virus after probing (Mauck et al. 2012). Ingwell et al. (2012) showed that $R$. padi aphids carrying barley yellow dwarf virus (BYDV, family Luteoviridae) preferred uninfected wheat plants, which promoted viral transmission. On the other hand, aphids that did not carry BYDV preferred BYDVinfected wheat plants, which promoted the acquisition of this virus. The switch of the aphids' preference from infected plants to uninfected plants after acquiring BYDV maximized the 
pathogen transmission potential (Ingwell et al. 2012). A similar observation was made for potato leaf roll virus (PLRV, family Luteoviridae) and its aphid vector Myzus persicae (Rajabaskar et al. 2014). M. persicae aphids that did not carry PLRV preferred infected potato plants, while M. persicaecarrying the virus preferred healthy plants. This switch in host plant preference seems to enhance virus acquisition and transmission. Both the BYDVand PLRV-induced changes in feeding preference can be a direct result of virus-vector interactions, but may also be affected by the exposure of the vector to infected plants (Rajabaskar et al. 2014).

Tomato spotted wilt virus (TSWV), a member of the only plant-infecting genus (Tospovirus) in the family Bunyaviridae, alters the feeding behaviour of its thrips vector Frankliniella occidentalis (order Thysanoptera) (Stafford et al. 2011). Male thrips infected with TSWV showed an up to threefold increase in feeding frequency compared with uninfected thrips. Infected male thrips made more non-ingestion probes (probes in which they salivate, but leave cells largely intact), which cause less damage to the plant cells. Functional plant leaf cells are very important for successful TSWV infection, and due to this altered feeding behaviour, infected males transmit TSWV in a more efficient manner than females (van de Wetering et al. 1998; Stafford et al. 2011).

Tomato yellow leaf curl virus (TYLCV, family Geminiviridae) was found to change the feeding behaviour of its whitefly vector Bemisia tabaci (order Hemiptera). Female whiteflies carrying TYLCV remained motionless for a longer time and moved slower after their first contact with the host plant (Moreno-Delafuente et al. 2013). In addition, probing and feeding behaviour changed. Plants are inoculated with virus during salivation in phloem sieve elements and this happens before phloem sap ingestion. B. tabaci carrying TYLCV fed more from phloem sieve elements and made more frequent phloem contacts. The duration of the salivation phase in the phloem sieve elements was also longer. All these behavioural changes are thought to enhance virus transmission.

\section{Arboviruses Change Mosquito and Midge Behaviour}

Many human and veterinary viruses use arthropods, such as mosquitoes, midges (both Diptera) or ticks (class Arachnida) as vectors. Arthropod-borne viruses (arboviruses) belong to various virus families and several have been found to change vector behaviour to increase virus transmission rates (van Houte et al. 2013; Lefèvre and Thomas 2008). These changes include 
alterations in feeding behaviour (probing time and/or frequency), locomotion and flight activity, and in mating behaviour.

Arboviruses classified in the family Bunyaviridae cause disease in their vertebrate host, but can also replicate in their insect vector. Many viruses from this family have been reported to change their vector's behaviour. Grimstad et al. (1980) first reported that the mosquito Aedes triseriatus, when infected orally with La Crosse virus (LACV), probed more on mice, with reduced rates of blood engorgement; a similar behavioural change was found by Jackson et al. (2012). LACV-infected Ae. triseriatus mosquitoes took a significantly smaller blood meal than uninfected mosquitoes, and the refeeding rate of LACV-infected Ae. triseriatus mosquitoes was twice as high as that of uninfected mosquitoes. The decreased blood meal size combined with increased refeeding potentially intensifies the number of contacts between virus-carrying insects and vulnerable animal hosts, thus enhancing horizontal transmission of LACV. The changes in blood feeding behaviour were accompanied by altered serotonin levels in the mosquito brain (Jackson et al. 2012). LACV is not the only animal-infecting virus from the family Bunyaviridae that can change vector feeding behaviour. Rift Valley fever virusinfected Culex pipiens mosquitoes also showed decreased feeding (Turell et al. 1985).

Many other arboviruses have been reported to alter vector feeding behaviour. Vesicular stomatitis virus (VSV, family Rhabdoviridae) infection significantly reduced the number of female Culicoides sonorensis midges that took a blood meal at 2 days post infection (d.p.i.), when the virus titer was at its highest (Bennett et al. 2008). Whether this behavioural change is beneficial for virus transmission has yet to be determined. Aedes aegypti mosquitoes infected with Dengue 3 virus (family Flaviviridae) required a significantly longer time to feed on a host to complete the blood meal than uninfected mosquitoes, mainly due to extended periods of probing, which is expected to enhance dengue virus transmission (Platt et al. 1997). In contrast, Putnam and Scott (1995) reported that Dengue 2 virus infection did not impair the feeding efficiency of Ae. aegypti, although the absence of an effect might be due to the infection method used (intrathoracic inoculation), the time of measurement, or the mosquito and virus strains used (maintained in the laboratory for a long time). Sindbis virus (SINV, family Togaviridae) infected Ae. aegypti females required more time for blood engorgement than uninfected ones. Meanwhile, infected mosquitoes also spent more time on blood meal localization (Qualls et al. 2012). A longer feeding period per meal potentially increases the virus transmission rate, but it could also impose a greater risk of death of the vector if detected by the blood donor, which may finally reduce virus transmission efficiency. 
Besides feeding behaviour, arboviruses manipulate other behavioural traits of their hosts, such as mating behaviour or locomotion/flight behaviour. Gabitzsch et al. (2006) described that the percentage of females inseminated by males was substantially higher for Ae. triseriatus females orally infected with LACV than for uninfected females. Similar results were found for $A e$. triseriatus females that had been infected with LACV by transovarial (vertical) transmission (Reese et al. 2009). Additionally, it was found that LACV-infected mosquitoes mated earlier in their life than uninfected ones. More interestingly, the virus titer was not correlated with increased insemination, but an earlier insemination of LACV-infected female mosquitoes might increase the number and percentage of virus progeny in the next mosquito generation, thus leading to enhanced virus transmission (Reese et al. 2009). The higher mating efficiencies might be achieved via an effect of LACV on sex pheromone expression in Ae. triseriatus (Reese et al. 2009).

Aedes aegypti mosquitoes infected with Dengue 2 virus showed up to a $50 \%$ increase in their locomotor activity compared with uninfected females (Lima-Camara et al. 2011). Whether this increase in locomotor activity leads to higher virus transmission needs further investigation. The authors speculate that modulation of the circadian clock is involved in inducing this alteration (Lima-Camara et al. 2011). In other cases a reduction in flight activity was seen, which might be a pathological consequence of the infection and is in fact less favourable for virus transmission. Female Culex tarsalis mosquitoes, for instance, showed a reduced flight activity when infected with Western equine encephalomyelitis virus (family Togaviridae) and fewer spontaneous flights were recorded (Lee et al. 2000). The idea that this virus has pathological consequences for the mosquito is reflected by a reduction in longevity, which was shown to be dependent on the viral load.

\section{Ecological and Evolutionary Aspects of Behavioural Manipulation}

How behavioural manipulation by viruses occurs and evolves is a central question, not only for virologists, but also for evolutionary biologists. Many evolutionary scenarios have been proposed that might underlie behavioural manipulation by parasites, including manipulation sensu stricto (selection of parasite genes that directly affect host behaviour), exploitation of host compensatory responses (selection of parasite genes on their pathological effects), mafia-like manipulation (selection of parasite genes on host collaborative behaviour) and manipulation by parasites with complex life cycles (selection of other parasite traits) 
(Lefèvre et al. 2009; Cézilly et al. 2010). However, experimental data supporting these theories are lacking and hard to obtain.

Viruses within the same family can target and modify the same host behavioural trait, e.g. locomotion or feeding behaviour, indicating that the ability to manipulate that trait might be conserved among this viral family or part thereof. For example, LACV, RVF and TSWV all belong to the family Bunyaviridae and all three can alter the feeding behaviour of their vector, suggesting that altered vector feeding behaviour might be a conserved trait among bunyaviruses (Jackson et al. 2012; Turell et al. 1985; Stafford et al. 2011). The baculoviruses BmNPV and AcMNPV both induce hyperactivity (Kamita et al. 2005; van Houte et al. 2012) and several baculoviruses including LdMNPV, AcMNPV, SeMNPV, MbNPV have been reported to induce tree-top disease in their lepidopteran hosts (Hoover et al. 2011; Ros et al. 2015; van Houte et al. 2014b; Goulson 1997). These data demonstrate that the ability of inducing hyperactivity and tree-top disease in lepidopteran hosts is, at least to some degree, conserved among baculoviruses.

Though the ability to modify the same behavioural trait might be conserved, different viruses may use different mechanisms to achieve this. For example, although both BmNPV and AcMNPV can induce hyperactivity in their hosts, the exact role of the encoded PTP protein might be different depending on the virus-host interaction. In BmNPV-infected B. mori larvae, the phosphatase activity of the PTP protein is not needed for the induction of hyperactivity (Katsuma et al. 2012). As deletion of the BmNPV ptp gene was shown to affect budded virus (BV) production in many different tissues including the larval brain, it was hypothesized that BmNPV PTP may exert its behavioural function as a structural component of the virus particles rather than as an enzyme. In contrast, in AcMNPV-infected $S$. exigua larvae the PTP phosphatase activity is required for induction of hyperactivity, since mutating the catalytic site of the encoded protein blocked induction of hyperactivity in S. exigua larvae, providing strong evidence that a host or viral protein is targeted by the enzymatic activity of the viral PTP to cause this behavioural change (van Houte et al. 2012). Another example of varying mechanisms can be seen in baculovirus-induced tree-top disease: both LdMNPV and AcMNPV can induce tree-top disease in their host; the egt gene from LdMNPV is involved in this behavioural change, while the egt gene from AcMNPV is not (Hoover et al. 2011; Ros et al. 2015).

Alternatively, unrelated viruses might use the same proximate mechanism to induce a similar host behavioural change, representing a case of convergent evolution (Ponton et al. 2006; van Houte et al. 2013). For example, both LbFV and HzNV-2 are present in the female host 
reproduction tract (of wasps and moths, respectively) and enhanced their transmission via affecting the reproduction behaviour of their host (Burand 2009; Varaldi et al. 2012).

Behavioural changes following infection by viruses can be adaptive to the host or to the parasite, or simply be pathological side effects of the infection (Thomas et al. 2005; van Houte et al. 2013). However, in many examples it is hard to exactly identify costs and benefits for both the parasite and the host. For the behavioural changes to be adaptive to the parasites, two features are critical: strong manipulation of the host and high prevalence within the host population (Lafferty and Kruis 2012). Some baculoviruses have a high prevalence within their host and a strong ability to induce behaviour changes (tree-top disease and hyperactivity), at least in laboratory settings. However, whether manipulation leads to enhanced viral transmission in the field needs further support from experimental field data. Greater dispersal of infected larvae can be interpreted as spreading virus over larger areas, thus increasing viral transmission, but can also be interpreted as removing infected individuals from uninfected conspecifics, which would be adaptive to the host. Death at elevated positions combined with liquefaction of host cadavers may spread the virus over larger areas and on lower foliage. However, liquefaction at elevated positions can also decrease virus transmission, because baculoviruses will be inactivated more quickly by increased exposure to UV light. Therefore, death at lower positions might lead to a higher infection rate in the next generation (viral progeny are better protected from UV light at lower positions), though the within-generation transmission might be reduced (due to limited spread of the virus) (Hamblin and Tanaka 2013). Downward movement was seen for OpbuNPV-infected O. brumata (Raymond et al. 2005; see above), where occlusion bodies were better protected from light on stems than on foliage.

An interesting question is to what extent laboratory studies can be used to study ecological scenarios. Behaviour is a complex phenotype, which not only relies on parameters of host and virus (such as genetic properties, physiological condition, virus dose and virulence), but may also depend on many environmental factors such as light, temperature, quality and availability of resources, and the presence of other parasites (de Bekker et al. 2014). Therefore, the environmental context should be taken into account when studying behavioural manipulation. Taking the baculovirus-induced behavioural changes as an example, it is known that behavioural changes like hyperactivity and tree-top disease are observed in nature (Goulson 1997; Hofmann 1891), but little is known to what extent environmental factors play a role in determining these phenotypes. However, it is known that the host plant species can affect insect susceptibility to baculoviruses and therefore, indirectly can affect insect host 
behaviour (Cory and Hoover 2006). To be able to study the genetic basis of baculovirusinduced behavioural changes in the laboratory, many environmental factors are usually standardized (see Figure 10.2 for a schematic view of a laboratory set-up used to measure hyperactivity and tree-top disease in lepidopteran larvae infected with baculoviruses).

Hyperactivity

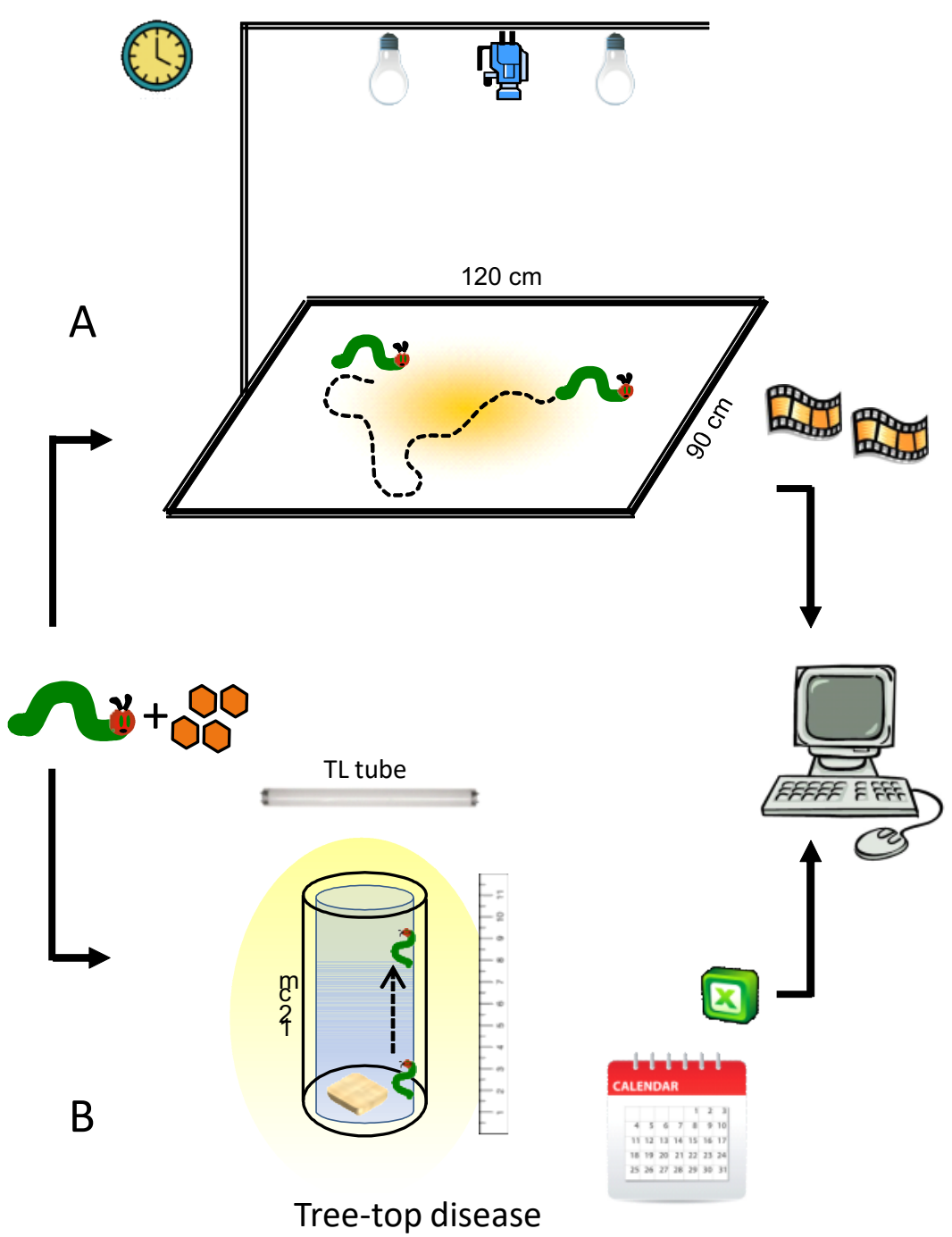

Figure 2. Laboratory settings used to study the genetic basis of baculovirus-induced behavioural changes in moth larvae (van Houte et al. 2014b; Ros et al. 2015; van Houte et al. 2012, 2014a). Larvae that were either mock infected or infected with wild type or mutant baculoviruses were used to study hyperactivity (a) or tree-top disease (b). (a) The system used for studying hyperactivity, consisting of a horizontal surface (arena) of $120 \times 90 \mathrm{~cm}$ equipped with a digital video camera positioned at $200 \mathrm{~cm}$ above the surface. The camera is flanked by two photography studio lights of $40 \mathrm{~W}$ each. The temperature in the arena was $24.5^{\circ} \mathrm{C} \pm 0.7$. To measure larval activity, individual larvae were placed in 
the arena and video-tracked for $10 \mathrm{~min}$. The total distance moved within this timeframe was calculated using EthoVision tracking software (Noldus Information Technology, The Netherlands). (b) The system used for studying tree-top disease consisting of sterile glass jars $(120 \mathrm{~mm}$ tall $\times 71 \mathrm{~mm}$ wide $)$ lined with mesh wire to facilitate climbing. A block of artificial diet (approximately $3.5 \mathrm{~cm}^{3}$ ) was placed on the bottom of each jar, and jars were closed with a metal lid containing small holes to allow ventilation. When light was applied only from above, jar walls were protected from light using aluminium foil and jars were covered with a piece of transparent plastic wrap containing three holes for ventilation. Jars were incubated at $27{ }^{\circ} \mathrm{C}$ with $50 \%$ relative humidity, and a $14 \mathrm{~L}: 10 \mathrm{D}$ photoperiod. Vertical position of the larvae was monitored twice per day, starting from 1 day post-infection until all larvae were dead or had pupated.

However, in nature, environmental conditions are constantly changing. An example is seen for LbFV infecting L. boulardi wasps. The distribution of LbFV, able to induce superparasitism in L. boulardi wasps is dependent on geographical location. The virus is highly prevalent in central populations of the $L$. boulardi distribution range, intermediately prevalent in marginal populations, and almost absent in newly established populations of $L$. boulardi (Patot et al. 2010). Though LbFV has a strong ability to induce superparasitism, few cases of superparasitism have been reported in newly established populations. However, in the central L. boulardi populations, superparasitism occurs more frequently, which might lead to high prevalence of the virus. It is predicted that superparasitism can complement imperfect vertical transmission of the virus (when the transmission rate is low) to maintain the virus at high frequencies (Gandon et al. 2006). Varaldi et al. (2012) even hypothesized that the superparasitism manipulation seen in the LbFV/L. boulardi system might be a common strategy among all parasitoid-associated viruses that infect the genital tract to maintain the virus in the host population. Without a certain level of horizontal transmission it is likely that the virus will die out eventually (assuming that vertical transmission never reaches $100 \%$ ).

Host manipulation by parasites can affect the ecological characteristics of host species by changing host population size, host mating system, the ecological community structures or food web structures. These effects can be substantial when the infected host displays a completely new behaviour or occupies a new niche. For example, the hairworm Gordionus chinensis induces water seeking behaviour in crickets (Tachycines spp.). Since this manipulation can be highly prevalent, the cricket cadavers can provide up to $60 \%$ of the energetic needs for the Kirikuchi charr (Salvelinus leucomaenis japonicas), a trout living in a local river in Japan (Sato et al. 2011). Even so, lepidopteran larvae infected with a baculovirus climb to the top parts of 
the canopy, which makes them visible for birds and other predators. This potentially will spread the virus over long distances. Dispersal of viral occlusion bodies by birds has been shown for Panolis flammea (Pf) MNPV-infected larvae of the pine beauty moth (Entwistle et al. 1993). If increased visibility to predators is a common phenomenon among baculovirus-infected caterpillar hosts, the behavioural change of "tree-top disease" may lead to changes in the food web structure.

Many insect hosts and vectors are of great medical, agricultural and environmental importance. Though the study of virus-induced behavioural manipulation is still in its infancy, this phenomenon has great potential in applied science, especially in the areas of pest management and vector control. As described above, altered behavioural traits, which can vary from slightly modified existing behavioural traits to completely new developed traits (van Houte et al. 2013), may have considerable impact on the insect population and on ecosystem dynamics. Understanding how viruses change insect behaviour may lead to broadly applicable strategies to modulate insect behaviour, facilitating regulation of insect population sizes and limiting virus transmission by vectors. This may be beneficial for agriculture, as well as human and veterinary health.

\section{Acknowledgements}

The authors thank Kelli Hoover for critically reading the manuscript, for useful suggestions and for sharing unpublished data. René van der Vlugt is acknowledged for careful reading of several sections prior to submission. Vera I.D. Ros was financed by a VENI-grant from the Netherlands Organisation for Scientific Research (project 863.11.017) 
1 Table 1: Overview of viruses and the described changes in insect host or vector behaviour

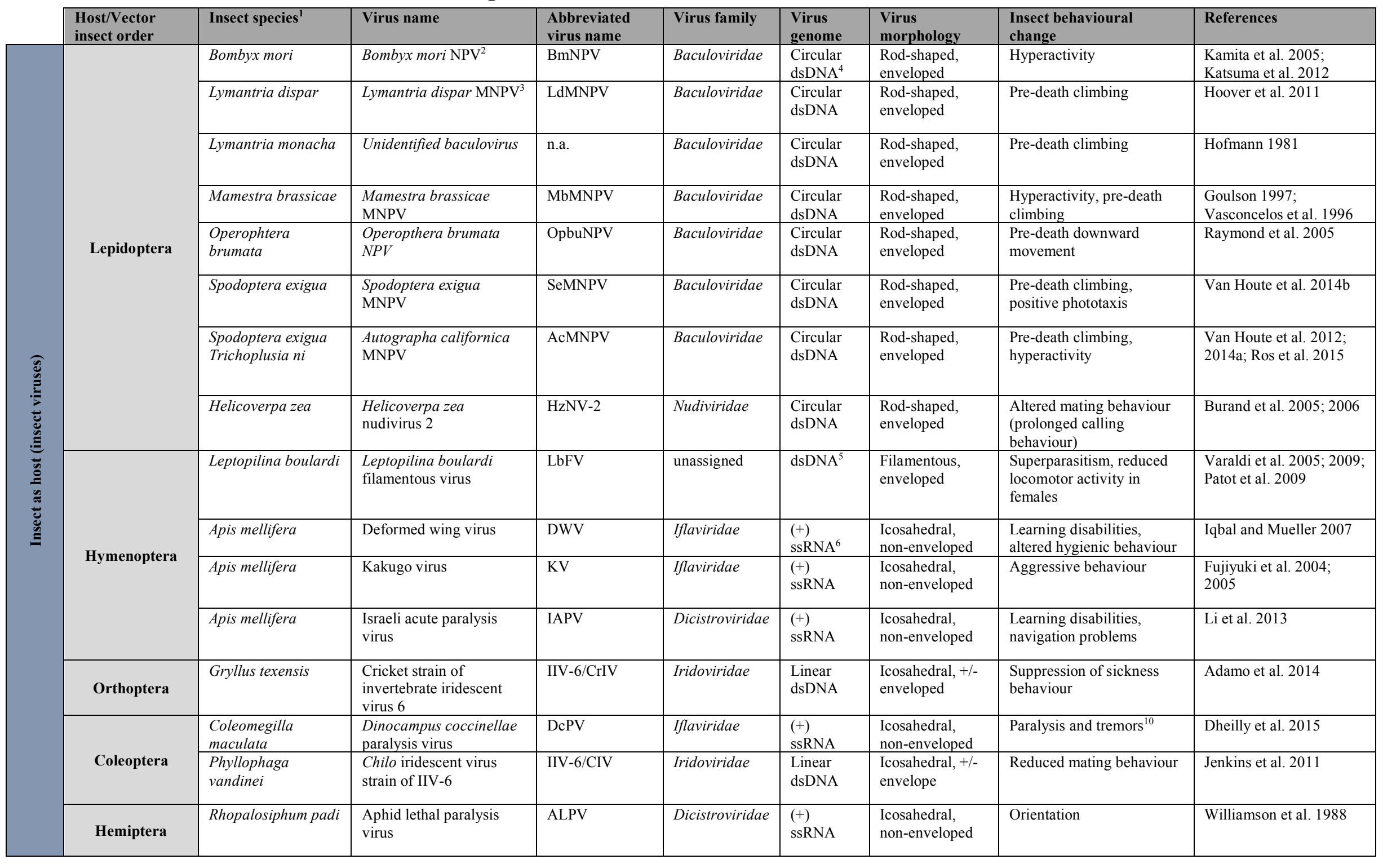




\begin{tabular}{|c|c|c|c|c|c|c|c|c|c|}
\hline & & Rhopalosiphum padi & $\begin{array}{l}\text { Rhopalosiphum padi } \\
\text { virus }\end{array}$ & $\mathrm{RhPV}$ & Dicistroviridae & $\begin{array}{l}(+) \\
\text { ssRNA }\end{array}$ & $\begin{array}{l}\text { Icosahedral, } \\
\text { non-enveloped }\end{array}$ & $\begin{array}{l}\text { Changes in aggregation } \\
\text { behaviour }\end{array}$ & Ban et al. 2008 \\
\hline \multirow{4}{*}{ 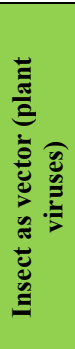 } & & Rhopalosiphum padi & Barley yellow dwarf virus & BYDV & Luteoviridae & $\begin{array}{l}(+) \\
\text { ssRNA }\end{array}$ & $\begin{array}{l}\text { Icosahedral, } \\
\text { non-enveloped }\end{array}$ & $\begin{array}{l}\text { Changes in plant } \\
\text { preference }\end{array}$ & Ingwell et al. 2012 \\
\hline & & Myzus persicae & Potato leaf roll virus & PLRV & Luteoviridae & $\begin{array}{l}+(+) \\
\text { ssRNA }\end{array}$ & $\begin{array}{l}\text { Icosahedral, } \\
\text { non-enveloped }\end{array}$ & $\begin{array}{l}\text { Changes in plant } \\
\text { preference }\end{array}$ & Rajabaskar et al. 2014 \\
\hline & & Bemisia tabaci & $\begin{array}{l}\text { Tomato yellow leaf curl } \\
\text { virus }\end{array}$ & TYLCV & Geminiviridae & $\begin{array}{l}\text { circular } \\
\text { ssDNA }^{7}\end{array}$ & $\begin{array}{l}\text { Icosahedral, } \\
\text { non-enveloped }\end{array}$ & $\begin{array}{l}\text { Change host feeding } \\
\text { behaviour }\end{array}$ & $\begin{array}{l}\text { Moreno-Delafuente et al. } \\
2013\end{array}$ \\
\hline & Thysanoptera & $\begin{array}{l}\text { Franklinella } \\
\text { occidentalis }\end{array}$ & Tomato spotted wilt virus & TSWV & Bunyaviridae & $\begin{array}{l}(+/-) \\
\text { ssRNA }^{8}\end{array}$ & $\begin{array}{l}\text { Spherical, } \\
\text { enveloped }\end{array}$ & Increased feeding rate & Stafford et al. 2011 \\
\hline \multirow{5}{*}{ 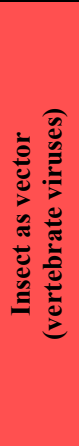 } & \multirow{5}{*}{ Diptera } & Aedes aegypti & Dengue virus & DENV & Flaviviridae & $\begin{array}{l}(+) \\
\text { ssRNA }\end{array}$ & $\begin{array}{l}\text { Spherical, } \\
\text { enveloped }\end{array}$ & $\begin{array}{l}\text { Prolonged feeding times, } \\
\text { increased locomotor } \\
\text { activity }\end{array}$ & $\begin{array}{l}\text { Platt et al. 1997; Lima- } \\
\text { Camara et al. } 2011\end{array}$ \\
\hline & & Aedes triseriatus & La Crosse virus & LACV & Bunyaviridae & $\begin{array}{l}+/-) \\
\text { ssRNA }\end{array}$ & $\begin{array}{l}\text { Spherical, } \\
\text { enveloped }\end{array}$ & $\begin{array}{l}\text { Increased re-feeding rate, } \\
\text { mating at earlier age }\end{array}$ & $\begin{array}{l}\text { Grimstad et al. 1980; } \\
\text { Jackson et al. 2012; } \\
\text { Reese et al. } 2009\end{array}$ \\
\hline & & Culex pipiens & Rift Valley fever virus & RVFV & Bunyaviridae & $\begin{array}{l}(+/-) \\
\text { ssRNA }\end{array}$ & $\begin{array}{l}\text { Spherical, } \\
\text { enveloped }\end{array}$ & Decreased feeding success & Turell et al. 1985 \\
\hline & & $\begin{array}{l}\text { Culicoides } \\
\text { sonorensis }\end{array}$ & Vesicular stomatitis virus & VSV & Rhabdoviridae & $\begin{array}{l}(-) \\
\text { ssRNA }\end{array}$ & $\begin{array}{l}\text { Bullet-shaped, } \\
\text { enveloped }\end{array}$ & Reduced feeding rates & Bennett et al. 2008 \\
\hline & & Culex tarsalis & $\begin{array}{l}\text { Western equine } \\
\text { encephalomyelitis virus }\end{array}$ & WEEV & Togaviridae & $\begin{array}{l}(+) \\
\text { ssRNA }\end{array}$ & $\begin{array}{l}\text { Spherical, } \\
\text { enveloped }\end{array}$ & Decreased flight activity & Lee et al. 2000 \\
\hline
\end{tabular}

1. Host/vector insect species for which behavioural changes have been described

2. Nucleopolyhedrovirus (NPV)

3. Multiple (M) NPV

4. Double-stranded DNA (ds DNA)

5. Positive single-stranded RNA: (+) ssRNA

6. Virus unassigned to family, but data indicate dsDNA virus (Patot et al. 2009)

7. Single-stranded DNA: ssDNA

8. Ambisense single-stranded RNA: (+/-) ssRNA

9. Negative single-stranded RNA: (-) ssRNA

10. $\mathrm{DcPV}$ is a virus of the parasitoid $D$. coccinellae, that uses DcPV as a 'biological weapon' to manipulate the behaviour of the ladybeetle C. maculate (see text) 


\section{Chapter}

Parasitic manipulation of host behaviour: Baculovirus SeMNPV EGT facilitates tree-top disease in Spodoptera exigua larvae by extending the time to death

\section{This chapter has been published as}

Han Y, van Houte S, Drees GF, van Oers MM and Ros VID, 2015. Parasitic manipulation of host behavior: baculovirus SeMNPV EGT facilitates tree-top disease in Spodoptera exigua larvae by extending the time to death. Insects 6, 716-731. 


\begin{abstract}
Many parasites enhance their dispersal and transmission by manipulating host behaviour. One intriguing example concerns baculoviruses that induce hyperactivity and tree-top disease (i.e., climbing to elevated positions prior to death) in their caterpillar hosts. Little is known about the underlying mechanisms of such parasite-induced behavioural changes. Here, we studied the role of the ecdysteroid UDP-glucosyltransferase (egt) gene of Spodoptera exigua multiple nucleopolyhedrovirus (SeMNPV) in tree-top disease in S. exigua larvae. Larvae infected with a mutant virus lacking the egt gene exhibited a shorter time to death and died before the induction of tree-top disease. Moreover, deletion of either the open reading frame or the ATG start codon of the egt gene prevented tree-top disease, indicating that the EGT protein is involved in this process. We hypothesize that SeMNPV EGT facilitates tree-top disease in $S$. exigua larvae by prolonging the larval time to death. Additionally, we discuss the role of egt in baculovirus-induced tree-top disease.
\end{abstract}




\section{Introduction}

A wide range of parasites are able to modify the behaviour of their hosts upon infection. Intriguing examples include the suicidal behaviour of the cricket Nemobius sylvestris when parasitized by the hairworm Paragordius tricuspidatus (Thomas et al., 2002) and the long-time hypokinetic stage of the cockroach Periplaneta americana when stung by the parasitoid jewel wasp Ampulex compressa (Kaiser and Libersat, 2015). Many parasite-induced behavioural changes are thought to enhance parasite reproduction, transmission and/or survival (Biron and Loxdale, 2013; Hughes, 2013; van Houte et al., 2013). Baculoviruses are large, double-stranded DNA viruses that induce behavioural changes in caterpillars. Infected host caterpillars show hyperactivity, which may spread viral progeny over larger areas (Goulson, 1997; Kamita et al., 2005; van Houte et al., 2012). Additionally, infected larvae present atypical climbing behaviour prior to death, leading to their migration to the top of plants. This pre-death climbing behaviour is described as "Wipfelkrankheit" or "tree-top disease" (Goulson, 1997; Hoover et al., 2011; Ros et al., 2015). The resulting increased visibility of infected insects to predators may enhance long-distance virus dispersal (Vasconcelos et al., 1996) and the final liquefaction of larval cadavers at elevated positions promotes virus dissemination over the foliage (Goulson, 1997).

Unravelling the underlying molecular mechanisms that drive baculovirus-induced host behavioural changes is the main focus of several recent studies. A significant study by Kamita et al. (2005) showed that the protein tyrosine phosphatase (ptp) gene from the baculovirus Bombyx mori nucleopolyhedrovirus (BmNPV) is involved in inducing hyperactivity in $B$. mori silkworms. Similarly, the Autographa californica multiple nucleopolyhedrovirus (AcMNPV) $p t p$ gene is required for induction of hyperactivity in Spodoptera exigua caterpillars (van Houte et al., 2012). Because both BmNPV and AcMNPV belong to the same taxon of baculoviruses (the Group I NPVs in the genus Alphabaculovirus), it is hypothesized that ptp-induced hyperactivity is a conserved strategy among Group I NPVs to enhance virus transmission (Hoover et al., 2011). Another study by Hoover et al. (2011) showed that the ecdysteroid uridine 5'-diphosphate (UDP)-glucosyltransferase (egt) gene from Lymantria dispar MNPV (LdMNPV) is required for triggering tree-top disease in gypsy moth larvae. However, it seems that the role of the egt gene in inducing tree-top disease varies between virus-host combinations. Unlike the egt gene from LdMNPV, the egt gene from AcMNPV is not needed for inducing tree-top disease in S. exigua and Trichoplusia ni larvae: when the egt gene was deleted from the AcMNPV genome, infected larvae still died at elevated positions (Ros et al., 2015). In addition, the egt gene seems not to be involved in the induction of hyperactivity: hyperactivity is still 
triggered in egt-negative BmNPV-infected B. mori larvae (Katsuma and Shimada, 2015). On the other hand, the AcMNPV ptp gene is not involved in inducing tree-top disease in S. exigua larvae (van Houte et al., 2014a). Therefore, AcMNPV-induced hyperactivity andtree-top disease are mediated by different mechanisms. Whether the baculovirus $p t p 2$ gene (van Houte et al., 2012), which is distantly related to the AcMNPV ptp gene, has a role in behavioural manipulation is not known.

During baculovirus infection, the encoded EGT enzyme inactivates the insect moulting hormone 20-hydroxyecdysone (20E) via the addition of a UDP-sugar group (either UDPglucose or UDP-galactose) (O'Reilly, 1995; O'Reilly and Miller, 1989). Due to the inactivation of the moulting hormone 20E, larval moulting is inhibited and infected larvae continue to eat. Finally, this may lead to a higher yield of viral progeny (O'Reilly and Miller, 1991). For several virus-host combinations, EGT has also been reported to extend the time to death of infected larvae. For example, 4 th and 5th instars of Spodoptera frugiperda infected with wild-type (WT) AcMNPV survived longer than larvae infected with a mutant AcMNPV lacking the egt gene (O'Reilly and Miller, 1991). However, many other host and virus factors also have an effect from the egt gene on host time to death (Cory et al., 2004; Ros et al., 2015). In another study involving $S$. exigua and T. ni larvae (3rd instars) the AcMNPV egt gene did not prolong the time to death, since larvae infected with WT AcMNPV or with an egt-deletion mutant did not differ significantly in the time to death (Bianchi et al., 2000; Ros et al., 2015).

The beet armyworm $S$. exigua is a polyphagous pest insect distributed worldwide. S. exigua MNPV (SeMNPV) is a specialist baculovirus that is highly infectious to its only host, S. exigua (Smits et al., 1987). A recent study by van Houte et al. (2011) showed that SeMNPV induced tree-top disease in S. exigua larvae. The induced tree-top disease was light-dependent as was shown in an experimental set-up: In glass jars containing mesh wire, infected larvae moved towards light prior to death. Larvae died at elevated positions (top parts in the jar) when light was applied from above, while larvae died at low positions (bottom of the jar) when light was applied from below. The vertical position of uninfected larvae was not light-dependent, since movement patterns of uninfected larvae in light and dark conditions were similar (Hoover et al., 2011).

In the current study, the role of the egt gene in SeMNPV-induced tree-top disease was analyzed. Virus-infected larvae were placed individually in the jars and the vertical positions of these larvae were monitored twice a day until they died. We show that WT-infected S. exigua larvae 
climbed up prior to death and died at elevated positions, while larvae infected with an SeMNPV mutant lacking the egt gene did not climb up prior to death. Moreover, we show that this difference can be explained by an earlier death of larvae infected with the egt-negative virus. These larvae succumb to the virus infection prior to the onset of climbing to elevated positions in WT-infected larvae. Therefore we hypothesize that in this virus-host system, EGT facilitates tree-top disease by prolonging the time to death.

\section{Experimental Section}

\section{Insect larvae}

Spodoptera exigua larvae were reared on artificial diet at $27^{\circ} \mathrm{C}$ with $50 \%$ relative humidity as described before (Smits et al., 1986), using a $14 \mathrm{~h}$ light/10 h dark photoperiod (7:00 lights on, 21:00 lights off).

\section{SeMNPV virus strains and generation of recombinant bacmids}

In this study, two SeMNPV WT viruses were used: a naturally occurring strain (SeMNPV G25, referred to as G25 WT) (Murillo et al., 2006) and viruses produced from the SeMNPV US1 strain-derived bacmid (see below; referred to as SeBac10 WT) (Pijlman et al., 2002). The SeBac10 WT bacmid was used to construct two SeMNPV mutants with deletions in the egt gene (Figure 1). The first mutant had a deletion of the major part of the egt open reading frame (ORF), ranging from nucleotide 40 to 1572 ( $\Delta$ egt-ORF), while in the second mutant only the start codon of the egt ORF was deleted ( $\Delta$ egt-ATG). Mutants were created following the protocol described by Ros et al. (2015), using primers 1 to 4 for the homologous recombination (Supplementary Table S1). The followed procedure first led to the replacement of the egt ORF or start codon by a chloramphenicol resistance gene (cat) flanked by modified loxP sites. Subsequently, the cat gene was removed by Cre-recombinase, leaving an inserted segment of 162 bp containing the recombined loxP site (Ros et al., 2015; Suzuki et al., 2005) (Figure 1). The deletion of the cat gene from both constructs was checked by PCR using primers 5 and 6 (for $\Delta e g t$-ORF) or 7 and 8 (for $\Delta e g t$-ATG) (Supplementary Table S1). 
G25 WT

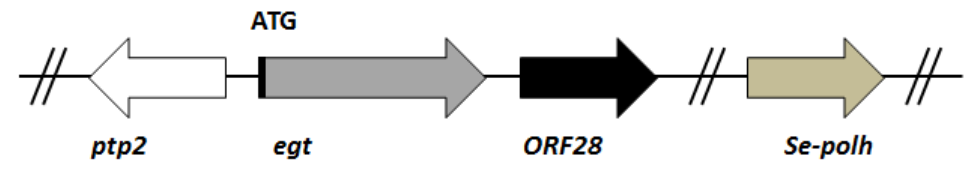

SeBac10 WT

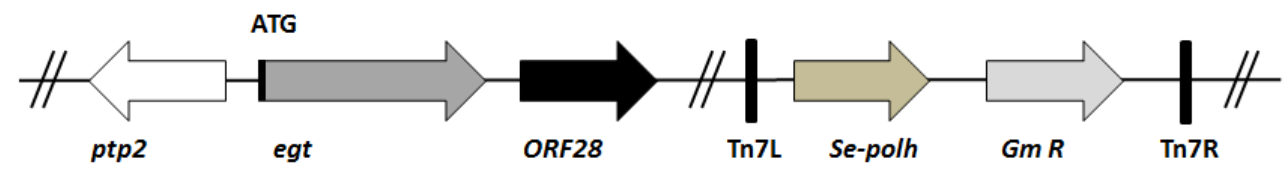

$\Delta e g t-O R F$

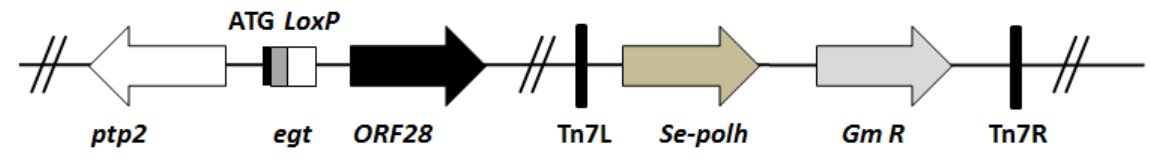

$\Delta e g t-A T G$

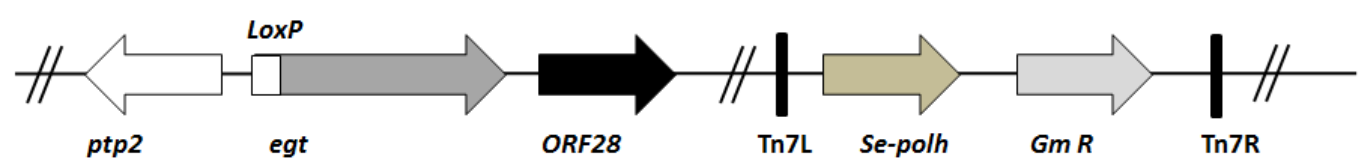

egt-repair

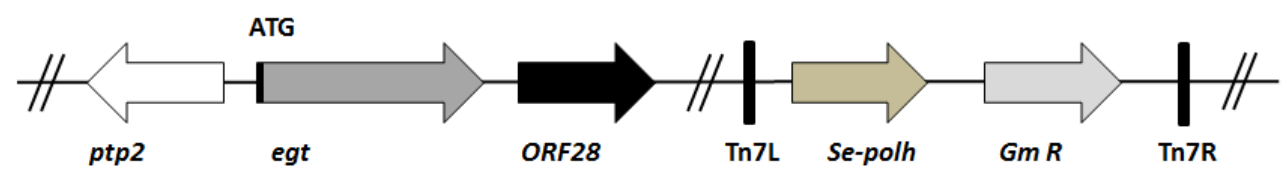

Figure 1. Overview of the recombinant bacmids used in this study. Two SeMNPV WT (G25 WT and SeBac10 WT) viruses were used: G25 WT is a naturally occurring strain and SeBac10 WT is a bacmid derived from the SeMNPV US1 strain. Using the SeBac10 WT bacmid, two SeMNPV mutants ( $(\Delta e g t-\mathrm{ORF}$ and $\Delta e g t$-ATG $)$ were made: in $\Delta e g t$-ORF, a major part of the egt ORF was replaced by a fragment containing a mutant loxP site (used to create the deletion); in $\Delta$ egt-ATG, only the start codon was replaced by the fragment with the loxP site. An egt-repair bacmid was also constructed. For all bacmid-derived viruses (which lack the original polyhedrin gene), the Se-polh gene and a gentamicin resistance gene ( $G m R)$ were inserted into the genomes between the left and right insertion sites, indicated as Tn7L and Tn7R, present in the bacmid. Positions of neighbouring genes (ptp2: protein tyrosine phosphatase 2 and ORF28) of egt are indicated.

After deletion of the target segments, the resulting bacmids were purified and used to transform E. coli $\mathrm{DH} 10 \Delta \mathrm{Tn} 7$ cells carrying the transposition helper plasmid pMON7124 (Luckow et al., 1993) (derived from E. coli DH10Bac $\Delta \operatorname{Tn} 7$ (Airenne et al., 2003) by removing the original bacmid). To enable oral infection of $S$. exigua larvae, the SeMNPV polyhedrin (polh) promoter and ORF were re-introduced into the SeBac10 WT (which lacks the polh gene), $\Delta$ egt-ORF and $\Delta e g t$-ATG genomes using the Bac-to-Bac transposition protocol (Luckow et al., 1993). To this aim, a modified pFastBacDual vector ( $\mathrm{pFBD}-$ Sepolh- $\Delta \mathrm{p} 10)$ was used as the donor vector. To generate this vector, the pFastBacDual vector (Invitrogen, Bleiswijk, the Netherlands) was first modified as described before by inserting the AcMNPV polh ORF downstream of the AcMNPV polh promoter and removing the $p 10$ promoter (Peng et al., 2010). Subsequently, the AcMNPV polh promoter and ORF were replaced by the SeMNPV polh promoter and ORF (corresponding 
to nucleotides 135474 to 776 of the SeMNPV genome (IJkel et al., 1999)) using the XhoI and Pst I restriction sites in the multiple cloning site.

To ensure that a possible phenotype of the $\Delta e g t$ viruses was not due to any other genome mutations, a repair virus (egt-repair) was constructed, using the $\Delta e g t$-ORF bacmid as a backbone (Figure 1). For the construction, a fragment corresponding to nucleotide 26091 to 28544 of the SeMNPV genome and covering the entire egt ORF was amplified with Phusion polymerase (Finnzymes, Fisher Scientific, Landsmeer, the Netherlands) using primers 9 and 10 (Supplementary Table S1). Purified PCR products $(100 \mathrm{ng} / \mu \mathrm{L})$ were mixed with $\Delta$ egt-ORF bacmid DNA $(500 \mathrm{ng} / \mu \mathrm{L})$ and Lipofectin transfection reagent (Invitrogen) in a 1:1:1 volume ratio and incubated at room temperature for $15 \mathrm{~min}$. The mixture was used to inject 4 th instars of $S$. exigua to facilitate generation of the egt gene repair virus via homologous recombination. To this end, each larva was injected with $10 \mu \mathrm{L}$ of the mixture using a Humapen Luxura insulin injection pen (Lilly, Houten, the Netherlands) and placed in a 12-well plate with a piece of artificial diet. The larvae were incubated at $27^{\circ} \mathrm{C}$ until the larvae had liquefied. Viral occlusion bodies (OBs) were purified from the cadavers and viral DNA was extracted as described before (Simón et al., 2008). Extracted viral DNA was used to transform E. coli cells and primers 5 and 6 (Supplementary Table S1) were used to screen the E. coli colonies for the presence of the correct repair bacmid via PCR.

\section{Generation, amplification and purification of viruses}

Bacmid DNA was isolated from E. coli DH10 $\operatorname{Tn} 7$ cells carrying the various bacmids (SeBac10 WT, $\Delta e g t$-ORF, $\Delta$ egt-ATG or egt-repair) using the PureLinK HiPure Plasmid Midiprep Kit (Invitrogen). Bacmid DNA (500 ng/ $\mu \mathrm{L}$ ) and Lipofectin transfection reagent (Invitrogen) were mixed in a 2:1 volume ratio. The mixture was incubated at room temperature for $15 \mathrm{~min}$ and used to inject 4th instars as described above. After injection, larvae were placed in a 12 -well plate with a piece of artificial diet and incubated at $27^{\circ} \mathrm{C}$ until the larvae liquefied. Liquefied larvae were ground and mixed with $10 \%$ sucrose solution containing $0.4 \%(\mathrm{w} / \mathrm{v})$ Patent Blue V Sodium salt colouring dye (Sigma-Aldrich, Zwijndrecht, the Netherlands). To amplify the obtained viruses, the sucrose-virus suspension was used to orally infect 3rd and 4th instars of S. exigua using droplet feeding as described before [6]. After liquefaction, larvae were ground in water and filtered through a double layer of cheese cloth. The suspension was centrifuged at $500 \times \mathrm{g}$ for $5 \mathrm{~min}$ to remove larval debris, after which the supernatant was centrifuged at $4000 \times g$ for $30 \mathrm{~min}$ to pellet the viral occlusion bodies (OBs). Finally, the OBs 
were resuspended in water and stored at $4{ }^{\circ} \mathrm{C}$. The concentration of OBs was counted using a Bürker-Türk haemocytometer (Marienfeld, Lauda-Königshofen, Germany).

\section{Infectivity assays}

Infectivity assays were performed to determine the infectivity for each virus, as described in Ros et al (2015). Late 2 nd instars of S. exigua were starved overnight for $16 \mathrm{~h}$ and allowed to moult during starvation. Newly moulted 3rd instars were selected and infected using droplet feeding as described in van Houte et al. (2012). Five viruses (G25 WT, SeBac10 WT, segtORF, $\Delta$ egt-ATG, egt-repair) were used to infect larvae and five different concentrations (6-fold dilutions: $1.0 \times 10^{3}, 6.0 \times 10^{3}, 3.6 \times 10^{4}, 2.0 \times 10^{5}$ and $1.3 \times 10^{6} \mathrm{OBs} / \mathrm{mL}$ ) were used for each virus (the dilutions were freshly prepared from a virus stock for each virus on the day of infection). For each virus concentration, 24-36 larvae were infected. Mock-infected larvae, which were droplet fed with a virus-free sucrose solution, were used as controls. Starting from two days post infection (dpi), larvae were scored for mortality until all larvae had died or had pupated. The assays were performed three times as three independent replicates. Data were analyzed using a logistic regression analysis in the program R v3.0.0 [10]. Treatment was used as a fixed effect and the model followed a binomial distribution. In addition, the $50 \%$ lethal concentration $\left(\mathrm{LC}_{50}\right)$ values were obtained using a logistic regression analysis in the program PoloPlus v1.0 (LeOra Software, 2002) (Robertson et al., 2007).

\section{Mortality assays}

Mortality assays were performed to determine the effect of each virus (G25 WT, SeBac10 WT, $\Delta e g t$-ORF, $\Delta e g t$-ATG, egt-repair) on the time to death of the infected larvae. 3rd instars of $S$. exigua were infected with a viral concentration of $10^{6} \mathrm{OBs} / \mathrm{mL}$ (known to kill at least $90 \%$ of infected larvae, the same concentration as used in the behavioural assays below), using droplet feeding as described above (van Houte et al., 2012). Mock-infected larvae were used as controls. Infected larvae were checked twice per day until they had died or had pupated (mock). For each virus, 36 larvae were infected and assays were performed three times as three independent replicates. The effects of the factors (treatment and experiment) on time to death were analyzed using Cox's proportional hazards model in the program R, as described in Ros et al. (2015). Since almost all larvae died as 3rd instars (or while moulting from 3rd to 4th instar), larval stage was not included as a factor in the model. In addition, mean time-to-death (MTD) values were calculated in $\mathrm{R}$. 


\section{Behavioural assays to measure tree-top disease}

Two behavioural assays, each with a different set of viruses, were done and each assay was performed twice as two independent replicates. In the first assay, larvae infected with the viruses G25 WT, SeBac10 WT, $\Delta$ egt-ORF or egt-repair were compared to investigate whether the egt gene plays a role in tree-top disease in S. exigua larvae. In the second assay, larvae infected with the viruses SeBac10 WT, $\Delta e g t$-ORF or $\Delta$ egt-ATG were compared to determine whether the EGT protein is required for tree-top disease. For all assays, newly moulted 3rd instars of S. exigua were infected with either a virus or a mock solution (containing no virus), using droplet feeding as described before (van Houte et al., 2012). For all viruses, a concentration of $10^{6} \mathrm{OBs} / \mathrm{mL}$ was used, a concentration known to kill at least $90 \%$ of infected larvae. For each assay, 30-40 larvae were infected (10 for the mock). Infected larvae were placed individually in glass jars (120 mm high and $71 \mathrm{~mm}$ in diameter) which were covered with a piece of transparent plastic Saran wrap (Supplementary Figure S4). Sterile mesh wire was placed in the jars to facilitate climbing and a piece of artificial food was placed at the bottom of the jars. Jar walls were protected from light using aluminum foil. Light was provided from above using three luminescent tube lamps of 18 Watts each placed at a $30 \mathrm{~cm}$ distance above the jars. Jars were placed at $27{ }^{\circ} \mathrm{C}$ with $50 \%$ relative humidity and a 14:10 LD photoperiod (7:00 lights on, 21:00 lights off). The vertical position of the larvae (highest point of the larval body) in the jars was monitored twice a day (mainly at 8:00-10:00 and 20:0022:00, for the exact time points see graphs) from one dpi until all larvae had died or pupated. Larvae that did not die due to viral infection were excluded from the data analyses.

The position at death was analyzed using a linear regression model $(\mathrm{lm})$ in the program R. Treatment (G25 WT, SeBac10 WT, $\Delta$ egt-ORF, $\Delta$ egt-AGT and egt-repair) and experiment (two replicates) were used as explanatory factors and it was determined whether these factors affected the positions at death. Since almost all larvae died as 3rd instars (or while moulting from 3rd to 4th instar), larval stage was not included as a factor.

\section{$R N A$ extraction and $R T-P C R$}

Newly moulted 3rd instars $S$. exigua were infected by droplet feeding with a viral concentration of $10^{6} \mathrm{OBs} / \mathrm{mL}$ (G25 WT, SeBac10 WT, $\Delta$ egt-ORF, $\Delta$ egt-ATG and egt-repair virus) as described before (van Houte et al., 2012). Mock-infected larvae were used as controls. At two dpi single larvae were homogenized in $250 \mu \mathrm{L}$ Trizol reagent (Invitrogen) and total RNA was isolated following the manufacturer's instructions. The RNA pellet was dissolved in $50 \mu \mathrm{L}$ 
water and heated for $10 \mathrm{~min}$ at $55^{\circ} \mathrm{C}$. Any contaminating DNA was removed with the DNAfree kit (Applied Biosystems, Life Technologies, Bleiswijk, the Netherlands). cDNA was produced using SuperScript III Reverse Transcriptase (Invitrogen) following the company's protocol. RT-PCR was performed, using primer pairs to amplify (A) a 468 bp sequence within the SeMNPV egt gene to check for the deletion of part of the egt ORF (primers 15 and 16, Supplementary Table S1), (B) a 899 bp sequence containing the egt start codon to check for the correct deletion of the start codon (forward primer annealed 6 to 27 bp upstream of the egt start codon, reverse primer annealed within the egt ORF) (primers 9 and 16, Supplementary Table S1), (C) a 492 bp sequence within the SeMNPV iel ORF to check for a successful virus infection (primers 13 and 14, Supplementary Table S1), and (D) a 486 bp sequence within the S. exigua host translation initiation factor eIF5A ORF (Van Oers et al., 1999) to check for successful RNA extraction and cDNA production for all larvae (primers 11 and 12, Supplementary Table S1). For each sample, a control sample was run in which the RT step was omitted (non-RT) to check for any DNA contamination. In addition, for each PCR a negative control with only water as template was processed.

\section{Results}

\section{Viral infectivity of the generated mutants}

To study whether deletion of the viral egt gene or of the egt start codon from the SeMNPV genome affected viral infectivity, we performed a logistic regression on the mortality data obtained after infection of $S$. exigua 3rd instars with G25 WT, SeBac10 WT, $\Delta$ egt-ORF, $\Delta$ egtATG or egt-repair viruses, for each replicate separately. For each virus, an odds ratio (relative potency) was determined: the ratio of infectivity of the respective virus compared to the G25 WT virus. In the first replicate, the infectivity of the WT virus differed from the infectivity of the mutant viruses ( $\Delta e g t$-ORF, $\Delta e g t$-ATG) and the egt-repair virus (judged by a lack of overlap of $95 \%$ confidence interval of the odds ratio and $p<0.001$; Supplementary Table S2), with the mutant and repair viruses being more virulent than the WT virus (Supplementary Table S2). However, this difference was absent in the other two replicates (odds ratios were not significantly different; Supplementary Table S2). In the second replicate, the repair was slightly more virulent than the G25 WT virus (Supplementary Table S2), but this effect was absent in the other two replicates. The $\mathrm{LC}_{50}$ value for each of the viruses is given in Supplementary Table 
S3. We chose to use a high virus concentration (killing at least $90 \%$ of all larvae) for infections in the behavioural assays, which came down to a concentration of $10^{6} \mathrm{OBs} / \mathrm{mL}$ for each virus.

\section{The SeMNPV egt gene extends the time to death of S. exigua larvae}

We investigated whether removal of the viral egt gene or of the egt start codon affected the time to death for 3rd instars of S. exigua, using a survival analysis. The model used (Cox's proportional hazards model) determines a mortality rate (hazard rate, rate at which larvae died) for the different values of the factors (treatment and experiment). The mortality rate was significantly affected by the treatment. The mortality rate for larvae infected with the mutant viruses was $2.04(\Delta e g t-\mathrm{ORF}, \mathrm{z}=5.00, p<0.001)$ and $1.40(\Delta e g t-\mathrm{ATG}, \mathrm{z}=2.41 ; p=0.016)$ times higher than for G25 WT-infected larvae. Similar ratios were found when compared to SeBac10 WT (1.90 for $\Delta e g t-\mathrm{ORF}, \mathrm{z}=4.52, p<0.001$ and 1.31 for $\Delta e g t-\mathrm{ATG}, \mathrm{z}=1.95, p=$ 0.052 ) or to egt-repair (2.37 for $\Delta e g t$-ORF, $\mathrm{z}=5.97, p<0.001$ and 1.63 for $\Delta e g t$-ATG, $\mathrm{z}=3.46$, $p<0.001)$. This is reflected in higher mean time to death (MTD) values for the G25 WT (73.84 h), SeBac10 WT (73.51 h) and egt-repair (76.48 h) viruses compared with the $\Delta$ egt-ORF (64.56 h) and $\Delta$ egt-ATG $(68.95 \mathrm{~h})$ viruses (Supplementary Table S3). There was no significant difference between the three replicates.

\section{Deletion of the egt gene prevents SeMNPV-induced tree-top disease}

To investigate the role of the SeMNPV egt gene in SeMNPV-induced tree-top disease, a behavioural assay was performed using 3rd instars of $S$. exigua infected with G25 WT, SeBac10 WT, $\Delta$ egt-ORF or egt-repair viruses. Both SeMNPV WT strains (G25 WT and SeBac10 WT) induced tree-top disease in S. exigua larvae. Infected larvae stayed at low positions until $52 \mathrm{~h}$ post infection (hpi) and from this time point on, larvae climbed up and finally died at elevated positions (Figure 2A,B and Supplementary Figure S2A,2B). When the egt ORF was deleted, infected larvae died at low positions (Figure 2C and Supplementary Figure S2C, black lines) $(\Delta$ egt-ORF $(n=52)$ versus G25 WT $(n=54)$ and SeBac10 WT $(n=56) ; T$-test $=3.859$ and 4.815 , respectively; d.f. $=216 ; p<0.001$ for both comparisons). The $\Delta e g t$-ORF mutant-infected larvae died earlier (from $43 \mathrm{hpi}$ ) than both WT-infected larvae (from $67 \mathrm{hpi}$ ), and in fact almost all were dead before the time point (52 hpi) at which WT-infected larvae started their upwards movement. Reintroducing the egt gene into the $\Delta e g t$-ORF genome (egt-repair) restored the WT phenotype, with egt-repair-infected larvae dying at similar positions (egt-repair $(n=59)$ versus G25 WT and SeBac10 WT; $T$-test $=-0.168$ and 0.764 , respectively; d.f. $=216$; $p=0.853$ and 0.446 , respectively) and at a similar time point as the WT-infected larvae (from 
67 hpi onwards) (Figure 2D and Supplementary Figure S2D, grey lines). Mock-infected larvae stayed at low positions throughout the experiment (Figure 2E and Supplementary Figure S2E). No significant differences were found between the two replicate experiments $(T$-test $=-0.012$; d.f. $=216 ; p=0.990)$.

\section{The EGT protein but not the egt transcript is involved in this process}

Deletion of the egt ORF may only affect EGT protein synthesis, but it may additionally affect neighbouring gene functioning. Moreover, a precursor miRNA encoding sequence has been predicted within the egt ORF from position nt 28046 to 28136 (database: Vir-Mir) (Li et al., 2007). A viral miRNA could play a role in the pathology of the virus or may regulate host physiology. We therefore created a mutant virus lacking only the egt start codon, but still containing the rest of the ORF, including the predicted precursor miRNA sequence. This allowed us to investigate whether the complete ORF deletion is needed for the observed differences in larval behaviour or whether this is also achieved when only the start codon is absent. In the $\Delta$ egt-ATG mutant, the ATG start codon was replaced by an insert of $162 \mathrm{bp}$ including the mutant $\operatorname{lox} P$ site (see Materials and Methods). This insert still allowed the egt transcript to be formed (as confirmed in Figure 4B), while translation of the EGT protein was inhibited. The next in-frame ATG codon is $223 \mathrm{bp}$ downstream of the deleted ATG. In the unlikely event that the next in-frame ATG would be used, 75 amino acids (aa) from the Nterminal end of the EGT protein would be removed (the egt gene is 524 aa in size, removal of 75 aa would be a considerable truncation of the functional protein). If the behavioural changes are observed for both mutants ( $\Delta e g t$-ORF and $\Delta e g t$-ATG), it is likely that the encoded EGT protein is involved. If changes are only observed for larvae infected with the $\Delta e g t$-ORF mutant, but not for larvae infected with the $\Delta$ egt-ATG mutant, there might be a role for the transcript, the encoded miRNA or even for side-effects on neighbouring genes.

Larvae infected with SeBac10 WT, $\Delta e g t$-ORF and $\Delta$ egt-ATG were compared. SeBac10 WTinfected larvae stayed at low positions until 56 hpi (Figure 3A and Supplementary Figure S3A). They displayed climbing behaviour prior to death and finally died at elevated positions. Larvae infected with $\Delta e g t$-ORF or $\Delta e g t$-ATG virus (Figure 3B,3C and Supplementary Figure S3B,S3C, black lines) did not climb up prior to death and died at low positions (SeBac10 WT $(n=66)$ versus $\Delta$ egt-ORF $(n=53)$ and $\Delta$ egt-ATG $(n=57) ; T$-test $=-7.109$ and -7.020 , respectively; d.f. $=172 ; p<0.001$ for both comparisons). These mutant-infected larvae died earlier (from 32 hpi onwards) than WT-infected larvae (from 56 hpi onwards). Because $\Delta e g t$-ORF and $\Delta e g t$ - 
ATG virus-infected larvae behaved similarly, we conclude that the EGT protein is required for tree-top disease.
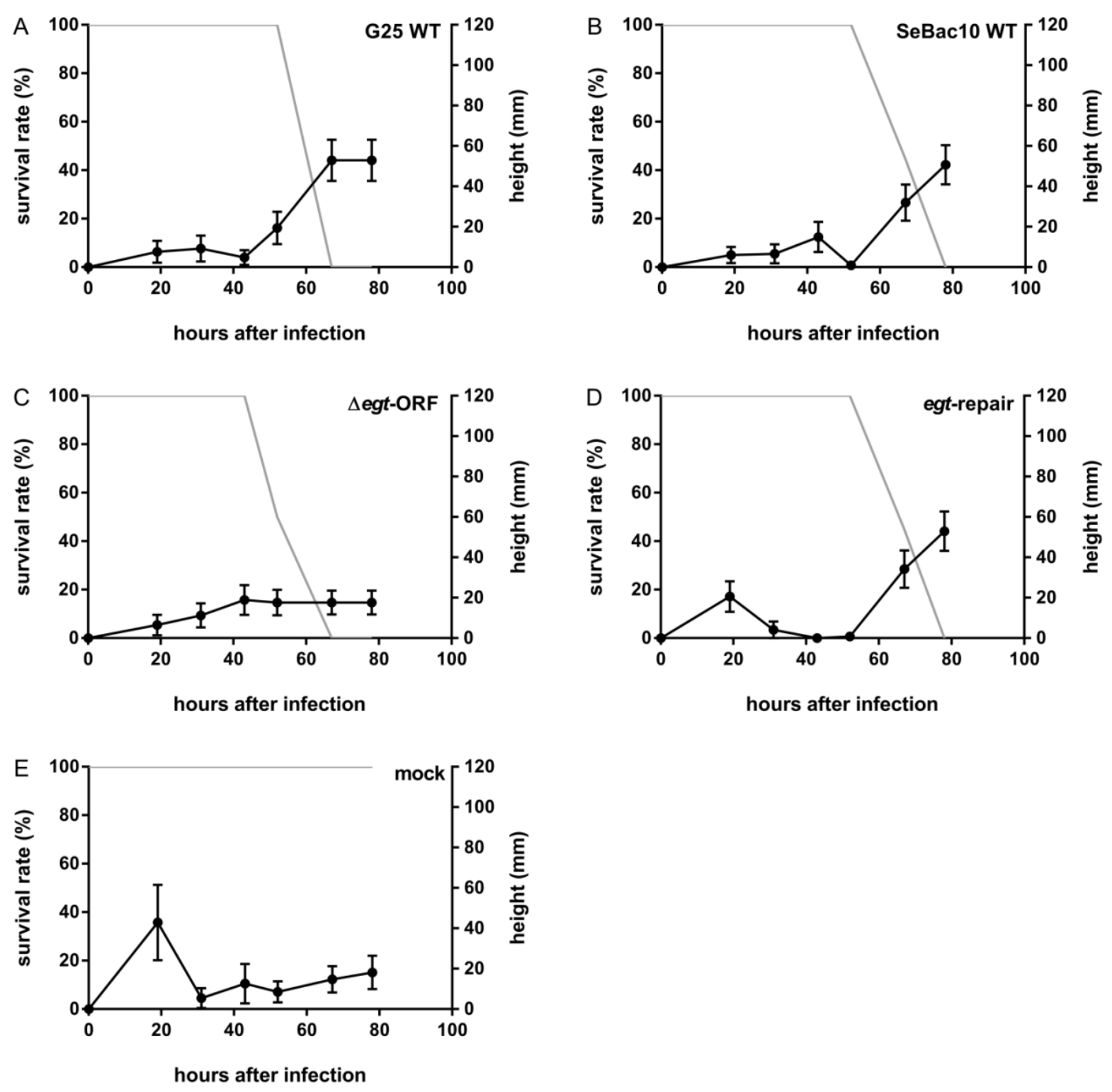

Figure 2. The effect of the deletion of the egt ORF on SeMNPV-induced tree-top disease in S. exigua larvae. Percentage surviving larvae (grey line) and height ( $\mathrm{mm}$ ) of larvae or cadavers (black line) were recorded at different time points after infection for 3rd instar S. exigua larvae infected with G25 WT (A, $n=25)$, SeBac10 WT (B, $n=27), \Delta e g t$-ORF $(\mathbf{C}, n=24)$, egt-repair $(\mathbf{D}, n=29)$ or mock $(\mathbf{E}, n=10)$. Error bars represent the standard error of the mean (SEM). 

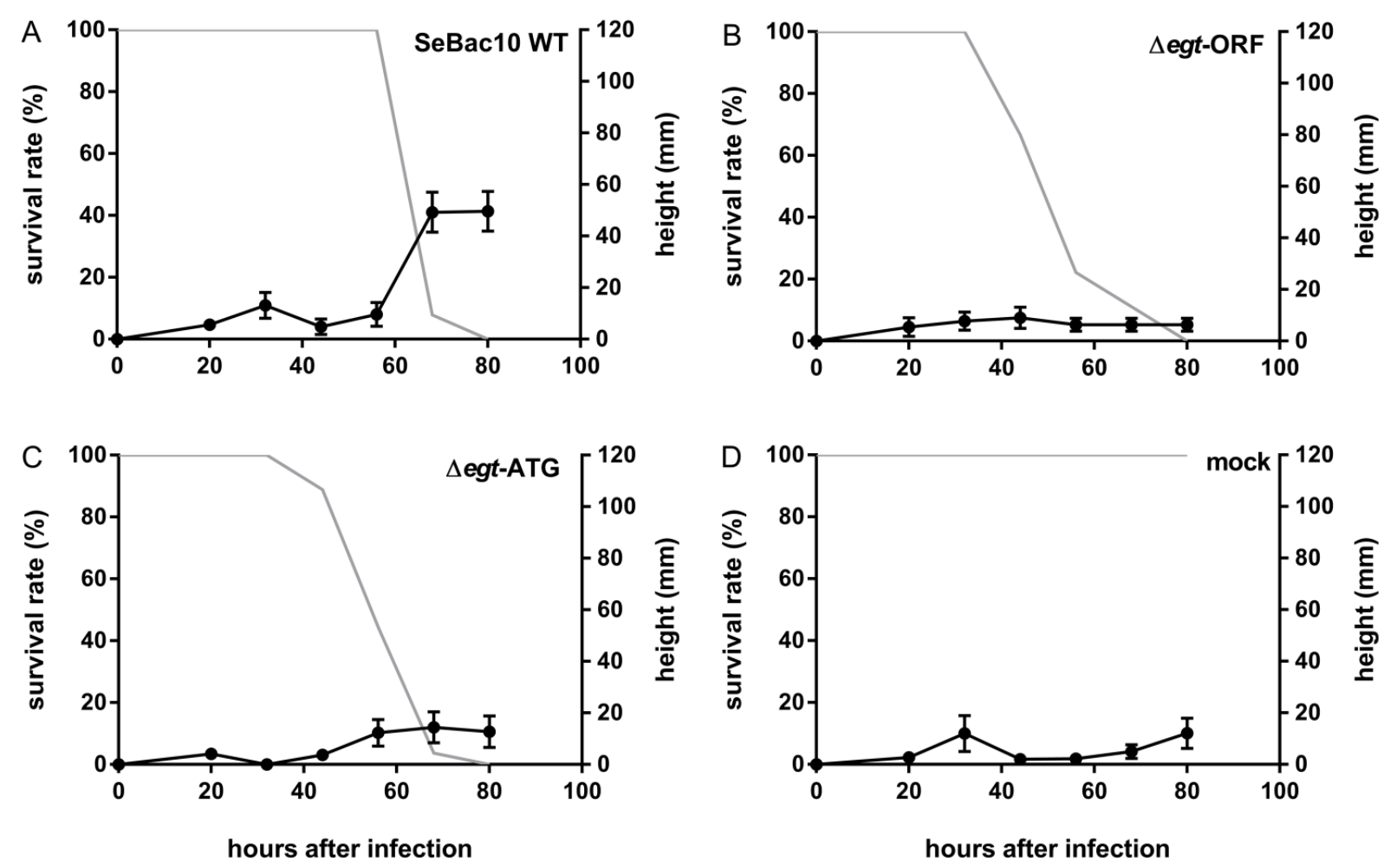

Figure 3. The effect of the deletion of the egt start codon on SeMNPV-induced tree-top disease in $S$. exigua larvae. Percentage surviving larvae (grey line) and height ( $\mathrm{mm}$ ) of larvae or cadavers (black line) were recorded at different time points after infection for 3rd instar $S$. exigua larvae infected with SeBac10 WT (A, $n=38), \Delta e g t$-ORF $(\mathbf{B}, n=27), \Delta e g t$-ATG $(\mathbf{C}, n=27)$ or mock $(\mathbf{D}, n=10)$. Error bars represent the standard error of the mean (SEM).

To confirm the expression of egt in WT- (G25 WT, SeBac10 WT), repair- (egt-repair) and $\Delta e g t$-ATG-infected larvae, and to confirm the absence of egt expression in $\Delta e g t$-ORF-infected larvae, an RT-PCR amplification was performed on total RNA extracted from mock- and virusinfected single whole larvae at two dpi (Figures 1 and 4). As expected, egt-specific mRNA was detected using primers annealing within the egt ORF (primers 15 and 16, Supplementary Table $\mathrm{S} 1$ ) in larvae infected with G25 WT, SeBac10 WT, $\Delta$ egt-ATG and egt repair viruses (Figure 4A; lanes 2-3 and 5-6), but was absent in mock- and $\Delta e g t$-ORF-infected larvae (Figure 4A, lanes 1 and 4). With primers annealing to the 5' upstream region of the egt ORF and to the $3^{\prime}$ end of the egt ORF (primers 9 and 16, Supplementary Table S1) it was shown that the ATG start codon of the egt gene was replaced by an insert of $162 \mathrm{bp}$ including the mutant loxP site in the $\Delta$ egt-ATG mutant, resulting in a slightly larger product (Figure 4B, lane 5) than for the WT and repair constructs (lanes 2-3 and 6). As expected, no product was seen for mock- and $\Delta$ egt-ORF-infected larvae (Figure 4B, lanes 1 and 4). An RT-PCR on the SeMNPV iel transcript was used as a control for virus infection and showed that the iel gene was indeed 
expressed in all virus-infected individuals, but not in the mock-infected larvae (Figure 4C). The host S. exigua eIF5A gene was expressed in all mock- and virus-infected larvae (Figure 4D), confirming successful RNA isolation and cDNA production.

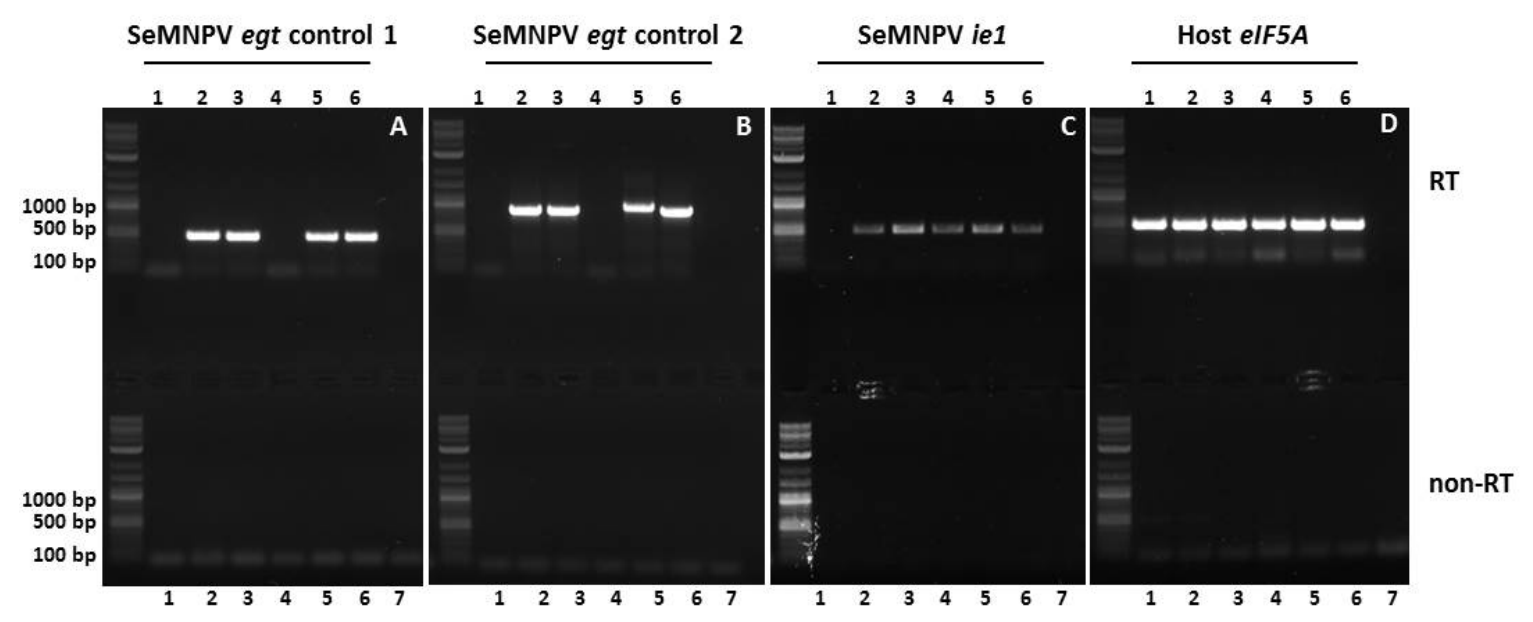

Figure 4. RT-PCR analysis of Spodoptera exigua larvae infected with WT, mutant and repair viruses. Larvae were mock-infected (1) or infected with G25 WT (2), SeBac10 WT (3), $\Delta$ egt-ORF (4), $\Delta$ egtATG (5) or egt-repair (6) viruses and processed for RT-PCR analysis at two dpi. For each PCR a water control (7) was included. The presence or absence of SeMNPV egt gene transcripts was checked by RTPCR (upper panels), using two different primer pairs, one pair annealing within the egt ORF (A) and the second pair annealing to the 5' upstream region of egt and within the egt ORF (B). Expression of the SeMNPV iel gene (C) was used as infection control and expression of the S. exigua eIF5A gene (D) was used as cDNA quality control. For each RT sample, a duplo sample without RT step (non-RT) was performed in parallel (lower panels). The 2-Log DNA Ladder (0.1-10.0 kb, New England BioLabs Inc., Leiden, the Netherlands) was used in the agarose gel to estimate PCR fragment sizes.

\section{Discussion}

Manipulation of host behaviour is a common strategy among parasites to enhance their survival and/or transmission (Biron and Loxdale, 2013; Hughes, 2013; van Houte et al., 2012). The underlying mechanisms of various examples of parasitic manipulation are now starting to be unraveled. It has been reported for more than a century that baculoviruses can induce tree-top disease in their lepidopteran hosts (Goulson, 1997; van Houte et al., 2013), but it was only in 2011 that the egt gene from LdMNPV was reported to be involved in this process in L. dispar 
larvae (Hoover et al., 2011). A recent study showed that the specialist baculovirus SeMNPV induces light-dependent tree-top disease in S. exigua larvae (van Houte et al., 2014b). The role of the SeMNPV egt gene in this process was, however, still unknown. In this study, we showed that the SeMNPV egt gene is required for the induction of tree-top disease in 3rd instars of $S$. exigua. Furthermore, we observed in our studies that the absence of tree-top disease in larvae infected with the egt-mutant viruses is a consequence of the fact that these larvae died earlier than WT-infected larvae, prior to the onset of the pre-death climbing behaviour.

Our study reveals that in SeMNPV-infected S. exigua larvae, EGT facilitates tree-top disease via prolonging the larval time to death. SeMNPV EGT extended the time to death of infected larvae, and in that extended life time the WT-infected caterpillars moved upwards, while the larvae infected with egt-mutants had already succumbed to virus infection. Previously, we showed that SeMNPV-induced tree-top disease is light-dependent and suggested that the virus might induce climbing by making use of host pathways involved in phototaxis and/or light perception (van Houte et al., 2014b). Whether SeMNPV EGT plays a further role in the induction of the light-dependent tree-top disease in S. exigua larvae remains undetermined. EGT has been reported to extend the host's life span in several virus-host systems, although in some systems no effect, or even a reduced time to death has been found (Cory et al., 2004; Katsuma and Shimada, 2015; Ros et al., 2015). Apparently, a variety of factors, including the virus-host interaction studied, the timing of infection, the developmental stage of the larvae and the viral concentration used, may have effects on the influence of the egt gene on host time to death (Cory et al., 2004; Katsuma and Shimada, 2015; Ros et al., 2015).

Strikingly, in AcMNPV-infected S. exigua larvae, tree-top disease appeared to be moultingdependent: only larvae that moulted during the course of infection climbed up and died at elevated positions (Ros et al., 2015). Those larvae that did not undergo moulting died at low positions. This observation appears to be specifically induced by AcMNPV in S. exigua larvae, since it was not observed in the current study with SeMNPV-infected S. exigua larvae and also not with AcMNPV in T.ni larvae (Ros et al., 2015). Here, most larvae died as 3rd instar or while moulting from 3 rd to 4 th instar and died at elevated positions.

We performed an additional experiment including a mutant SeMNPV virus lacking only the egt start codon ( $\Delta e g t$-ATG). This experiment showed, combined with the observation that both the egt ORF and the egt start codon mutant reduced the time to death and prevented tree-top disease in infected larvae, that the EGT protein is most likely responsible for extending the time to 
death and allowing pre-death climbing behaviour. It has been shown that behavioural changes in insects can be influenced by miRNA pathways or miRNA levels (Bartel, 2004) and it has been reported that miRNAs might be involved in regulating behaviour in Drosophila (Kadener et al., 2009). Similarly, viruses might affect host behaviour using viral miRNAs. A precursor miRNA encoding sequence has been predicted within the egt ORF [32]. However, our data indicate that the egt transcript is not involved in the altered phenotype. The RT-PCR results demonstrated that the egt transcript was still formed and the region for which a miRNA was predicted was still intact in this mutant. Whether the miRNA encoded in the egt gene is produced and whether it has a regulating role in the infection process must still be investigated.

The egt gene is present in almost all lepidopteran baculoviruses except in one clade of granuloviruses (Ros et al., 2015). Several studies have now investigated the role of egt in baculovirus-induced tree-top disease. Although the effects of egt on the occurrence of tree-top disease have been observed (Hoover et al. (2011) and this study), it is absent in other systems (Ros et al. (2015)) and its exact role remains unclear. Possibly, egt exerts its effect on tree-top disease by affecting the time to death and/or by inhibiting moulting-related climbing behaviour (2015). In the current study, egt extends the time to death, and egt-mutant-infected larvae do not reach the time point at which in WT-infected larvae tree-top disease is observed. Apart from affecting the time to death of larvae, egt is also known to suppress host moulting (O'Reilly, 1995; O'Reilly and Miller, 1989). Larval moulting is often accompanied by changes in behaviour, and by affecting moulting, egt might also change moulting-related behaviour. An example was seen in AcMNPV-infected 3rd instars of T. ni and S. exigua, where egt did affect moulting-related climbing behaviour but not tree-top disease, which occurred much later than the moulting-related climbing (Ros et al., 2015). If the death of larvae occurred around the time that larvae moult, or are about to moult, egt might, by having an inhibitory effect on moulting, also affect moulting-related behaviour, which can give the appearance that egt induces tree-top disease. This might be the case in LdMNPV-infected L. dispar larvae (Hoover et al., 2011; Ros et al., 2015). Lymantria dispar larvae feed in the tree canopy and move downwards to moult. In LdMNPV WT-infected larvae moulting was suppressed and larvae died up in the tree, while larvae infected with the LdMNPV $\Delta e g t$ virus moved downwards to moult and died at low positions. In the case of LdMNPV, the apparent effect of egt on tree-top disease might be a consequence of its inhibitory effect on moulting and on moulting-related behaviour. 


\section{Conclusions}

In this study we examined the role of the egt gene in SeMNPV-induced tree-top disease. We found that larvae infected with a mutant virus lacking the egt gene presented a shorter time to death and died before the onset of pre-death climbing behaviour. Moreover, deletion of either the open reading frame or the ATG start codon of the egt gene prevented tree-top disease, indicating that the EGT protein is required for the occurrence of tree-top disease. We observed that EGT facilitates the pre-death climbing behaviour by prolonging the larval time to death. Most likely, additional viral genes are needed to induce tree-top disease.

\section{Acknowledgments}

We thank Berndjan Eenink for his assistance with generating the $\Delta$ egt-ATG recombinant bacmid and Lisa Pennemann for her help with generating and amplifying the $\Delta$ egt-ATG recombinant SeMNPV. Els Roode's help in rearing the larvae was highly appreciated. We thank Amaya Serrano for providing the G25 WT isolate and Lia Hemerik for assistance with the statistical analyses. Amaya Serrano and Gorben Pijlman are acknowledged for useful discussions throughout the experiments. SvH and VIDR were supported by the Program Strategic Alliances of the Royal Dutch Academy of Sciences (project 08-PSA-BD-01) and VIDR is supported by a VENI grant of the Netherlands Organization for Scientific Research (project 863.11.017). 


\section{Supplementary Materials}

Table S1. Primers used in this study.

\begin{tabular}{|c|c|c|c|}
\hline Number & Primer Name & Sequence $\left(5^{\prime}-3^{\prime}\right)$ & Function \\
\hline 1 & $\Delta e g t$-ORF-Fw & $\begin{array}{c}\text { CATCGGTCACCATGAACGGTTGCGCTGTCCTAATTTTATTTTTTGCACT } \\
\text { GGCTCGGATCCACTAGTAACGG }\end{array}$ & $\begin{array}{l}\text { Primer with } 50 \text { bp overhang (italics) to amplify a product for } \\
\text { homologous recombination to remove part of the egt ORF }\end{array}$ \\
\hline 2 & $\Delta e g t-\mathrm{ORF}-\mathrm{Rv}$ & $\begin{array}{l}\text { ATTTAGGGTTAAATTACATGGTTCATACATACACACACATCCTGTTTTTT } \\
\text { CCTCTAGATGCATGCTCGAG }\end{array}$ & $\begin{array}{l}\text { Primer with } 50 \text { bp overhang (italics) to amplify a product for } \\
\text { homologous recombination to remove part of the egt ORF }\end{array}$ \\
\hline 3 & $\Delta e g t-\mathrm{ATG}-\mathrm{Fw}$ & $\begin{array}{c}\text { TTTAGGTCACCCATTTACTGTATCGAATCATCGGTCACCGCTCGGATC } \\
\text { CACTAGTAACG }\end{array}$ & $\begin{array}{l}\text { Primer with } 50 \mathrm{bp} \text { overhang (italics) to amplify a product for } \\
\text { homologous recombination to remove the egt start codon }\end{array}$ \\
\hline 4 & $\Delta e g t-A T G-R v$ & $\begin{array}{c}\text { GCCGAACCCGTGGTCAGTGCAAAAAATAAAATTAGGACAGCGCAACC } \\
\text { GTTCCTCTAGATGCATGCTCG }\end{array}$ & $\begin{array}{l}\text { Primer with } 50 \text { bp overhang (italics) to amplify a product for } \\
\text { homologous recombination to remove the egt start codon }\end{array}$ \\
\hline 5 & $\Delta e g t$-ORF-check Fw & ATGTGTGCTCTTCGTCAGATG & To check the $\Delta$ egt-ORF deletion mutant \\
\hline 6 & $\Delta e g t$-ORF-check Rv & TATTGCCTACGCGCGC & To check the $\Delta$ egt-ORF deletion mutant \\
\hline 7 & $\Delta e g t$-ATG-check Fw & GGCTAAACCGATGTTGTAGTG & To check the $\Delta$ egt-ATG deletion mutant \\
\hline 8 & $\Delta e g t$-ATG-check Rv & CCCGGTACCTCACACTAAATTAATTCTCAGTAATTGAC & To check the $\Delta$ egt-ATG deletion mutant \\
\hline 9 & egt-repair-Fw & ATTTACTGTATCGAATCATCGG & $\begin{array}{l}\text { To amplify a product for homologous recombination to generate } \\
\text { the egt-repair virus, for RT-PCR on SeMNPV egt and to check } \\
\text { egt-ATG deletion }\end{array}$ \\
\hline 10 & egt-repair-Rv & GGGTTAAATTACATGGTTCATACA & $\begin{array}{l}\text { To amplify a product for homologous recombination to } \\
\text { generate the egt-repair virus }\end{array}$ \\
\hline 11 & Se-eIF $5 A-\mathrm{Fw}$ & GCCATGGCTGACATCGAGGATAC & RT-PCR on S. exigua eIF5A \\
\hline 12 & Se-eIF $5 A$-Rv & GCGGTACCGGTTTATTTGTCGAGAGC & RT-PCR on S. exigua eIF5A \\
\hline 13 & Se-iel Fw & GACAAGAATGACGATGATATCGG & RT-PCR on SeMNPV ie1 \\
\hline 14 & Se-iel Rv & GGACAATTGCTTTTCCGAAAAC & RT-PCR on SeMNPV iel \\
\hline 15 & Se-egt-ORF-Fw & CAAGAGGTTGATTGACGAACAA & RT-PCR on SeMNPV egt and to check egt-ORF deletion \\
\hline 16 & Se-egt-ORF-Rv & AGCGATTTGGGATGTTTGTC & $\begin{array}{l}\text { RT-PCR on SeMNPV egt and to check egt-ORF and } \\
\text { egt-ATG deletion }\end{array}$ \\
\hline
\end{tabular}


Table S2. Outcome of the logistic regression analysis of the infectivity assays. Per replicate, an odds ratio (relative potency) was determined for each virus: the ratio of the infectivity of the respective virus relative to the infectivity of the G25 WT virus. The upper and lower limits of the $95 \%$ confidence interval are also given, as well as the $P$ value.

\begin{tabular}{|c|c|c|c|c|c|}
\hline \multirow[t]{2}{*}{ Replicate } & \multirow[t]{2}{*}{ Viruses } & \multirow[t]{2}{*}{ Odds Ratio } & \multicolumn{2}{|c|}{ 95\% Confidence Interval } & \multirow[t]{2}{*}{ P value } \\
\hline & & & Low & High & \\
\hline \multirow[t]{5}{*}{1} & G25 WT & 1.000 & - & - & - \\
\hline & SeBac10 WT & 1.634 & 0.988 & 2.715 & 0.057 \\
\hline & $\Delta$ egt-ORF & 2.654 & 1.601 & 4.440 & $<0.001 * * *$ \\
\hline & $\Delta e g t-\mathrm{ATG}$ & 5.989 & 3.542 & 10.292 & $<0.001 * * *$ \\
\hline & egt-repair & 3.845 & 2.300 & 6.509 & $<0.001 * * *$ \\
\hline \multirow[t]{5}{*}{2} & G25 WT & 1.000 & - & - & - \\
\hline & SeBac10 WT & 1.291 & 0.774 & 2.158 & 0.329 \\
\hline & $\Delta$ egt-ORF & 0.680 & 0.407 & 1.132 & 0.139 \\
\hline & $\Delta e g t-\mathrm{ATG}$ & 1.135 & 0.679 & 1.902 & 0.629 \\
\hline & egt-repair & 1.759 & 1.046 & 2.974 & $0.034 *$ \\
\hline \multirow[t]{5}{*}{3} & G25 WT & 1.000 & - & - & - \\
\hline & SeBac10 WT & 1.330 & 0.786 & 2.256 & 0.288 \\
\hline & $\Delta$ egt-ORF & 0.597 & 0.349 & 1.016 & 0.058 \\
\hline & $\Delta e g t-\mathrm{ATG}$ & 1.607 & 0.946 & 2.745 & 0.080 \\
\hline & egt-repair & 0.899 & 0.531 & 1.521 & 0.691 \\
\hline
\end{tabular}

Table S3. Dose-mortality response ( $\log \mathrm{LC}_{50}$ ) and mean time to death (MTD) of 3rd instar S. exigua larvae infected with G25 WT, SeBac10 WT, $\Delta$ egt-ORF, $\Delta$ egt-ATG or egt-repair virus. MTD was determined for a virus concentration of $10^{6} \mathrm{OBs} / \mathrm{mL}$ (approximately $90 \%-95 \%$ mortality).

\begin{tabular}{|c|c|c|c|c|c|c|}
\hline \multirow[t]{2}{*}{ Virus } & \multirow[t]{2}{*}{$\begin{array}{c}\log \mathrm{LC}_{50} \\
(\mathrm{OBs} / \mathrm{mL})\end{array}$} & \multicolumn{2}{|c|}{$\begin{array}{c}\text { Log } 95 \% \text { Confidence } \\
\text { Interval }(\mathrm{OBs} / \mathrm{mL})\end{array}$} & \multirow[t]{2}{*}{$\begin{array}{c}\text { MTD } \\
\text { (h) }\end{array}$} & \multicolumn{2}{|c|}{$\begin{array}{l}\text { 95\% Confidence } \\
\text { Interval (h) }\end{array}$} \\
\hline & & low & high & & low & high \\
\hline G25 WT & 4.70 & 4.44 & 4.97 & 73.84 & 71.87 & 75.81 \\
\hline SeBac10 WT & 4.42 & 4.16 & 4.67 & 73.51 & 71.75 & 75.28 \\
\hline$\Delta e g t-\mathrm{ORF}$ & 4.64 & 4.40 & 4.88 & 64.56 & 62.43 & 66.68 \\
\hline$\Delta e g t-\mathrm{ATG}$ & 4.08 & 3.84 & 4.30 & 68.95 & 66.74 & 71.16 \\
\hline egt-repair & 4.26 & 3.98 & 4.53 & 76.48 & 74.66 & 78.29 \\
\hline
\end{tabular}




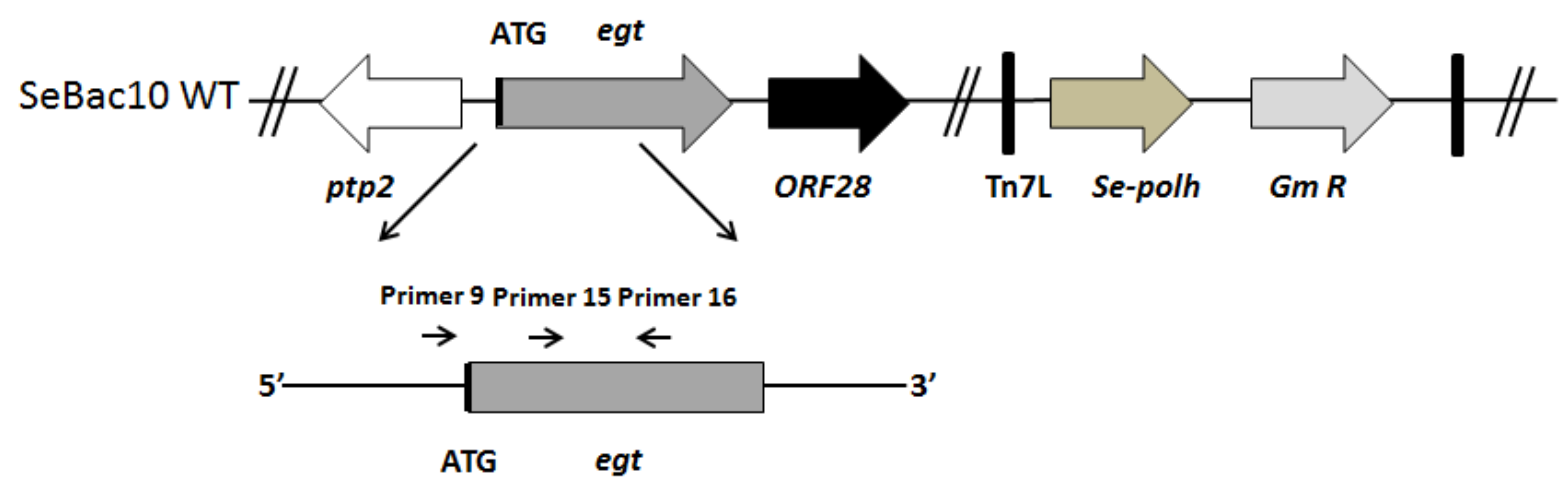

Figure S1. Position of the primers (horizontal arrows) that were used to check the deletion of the egt ORF or ATG start codon. Primers 15 and 16 anneal within the egt ORF and were used to check the deletion of the egt ORF. Primer 9 (anneals 27 bp upstream of the egt start codon) and primer 16 (anneals within the egt $\mathrm{ORF}$ ) were used to check the deletion of the egt start codon.
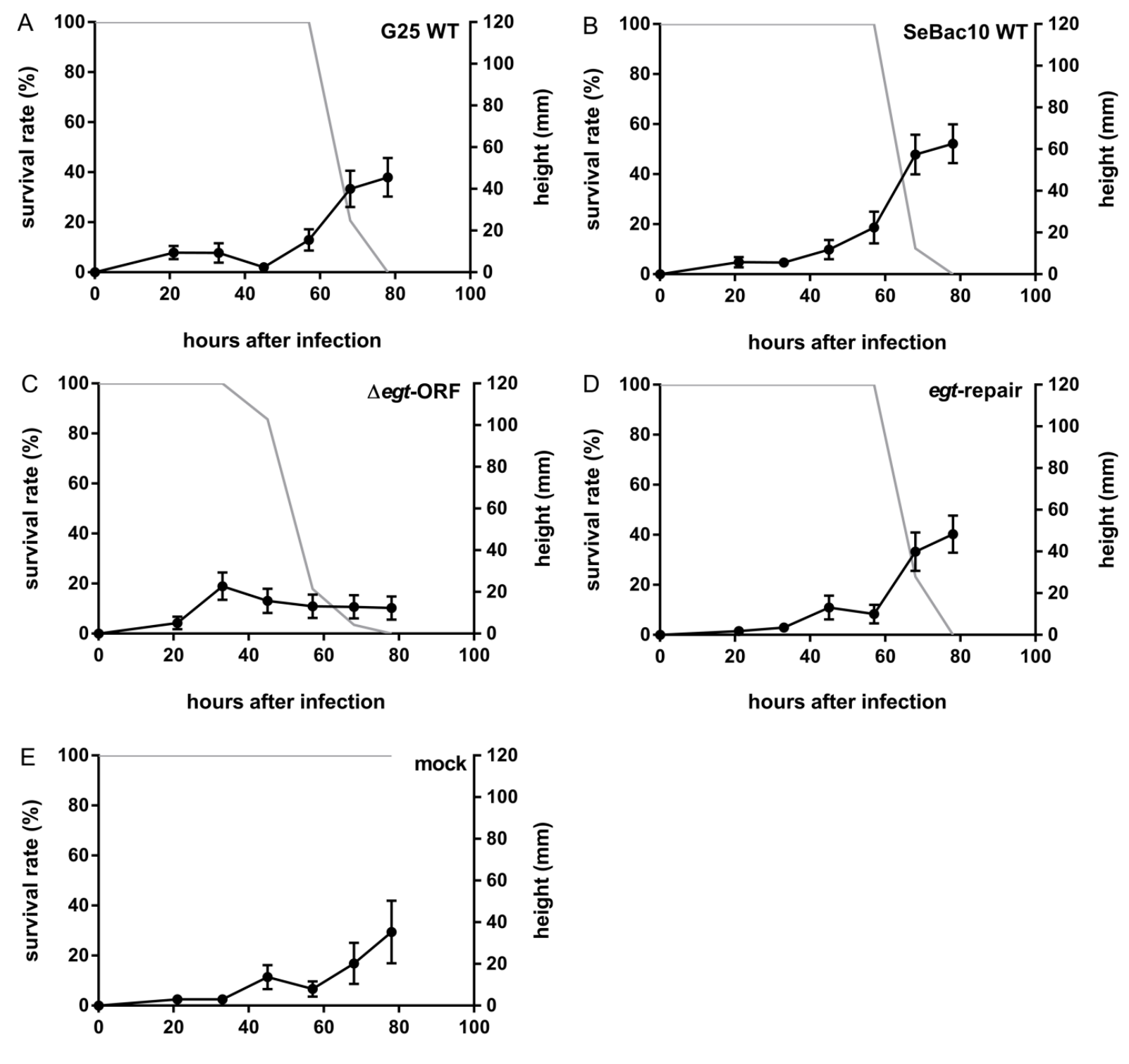

hours after infection 
Figure S2. Repetition of the experiment presented in Figure 2. The effect of the deletion of the egt ORF on SeMNPV-induced tree-top disease in S. exigua larvae. Percentage surviving larvae (grey line) and height $(\mathrm{mm})$ of larvae or cadavers (black line) were recorded at different time points after infection for 3rd instar S. exigua larvae infected with G25 WT (A, $n=29)$, SeBac10 WT (B, $n=29)$, segt-ORF (C, $n=28)$, egt-repair ( $\mathrm{D}, n=30)$ or mock $(\mathrm{E}, n=10)$. Error bars represent the standard error of the mean (SEM).
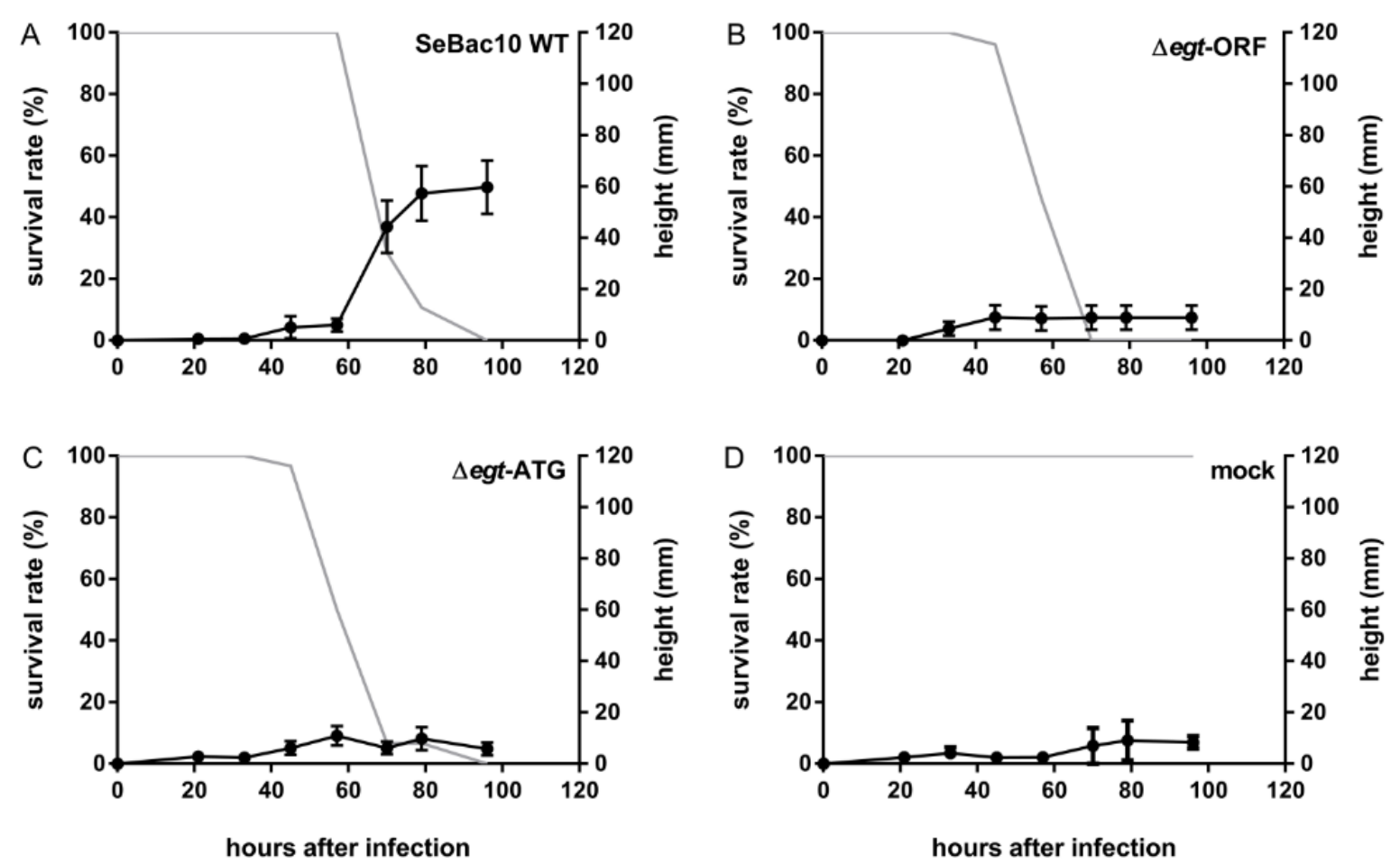

Figure S3. Repetition of the experiment presented in Figure 3. The effect of the deletion of the egt start codon on SeMNPV-induced tree-top disease in S. exigua larvae. Percentage surviving larvae (grey line) and height $(\mathrm{mm})$ of larvae or cadavers (black line) were recorded at different time points after infection for 3rd instar S. exigua larvae infected with SeBac10 WT (A, $n=28), \Delta e g t$-ORF (B, $n=26), \Delta e g t$-ATG $(\mathbf{C}, n=30)$ or mock $(\mathbf{D}, n=10)$. Error bars represent the standard error of the mean (SEM). 


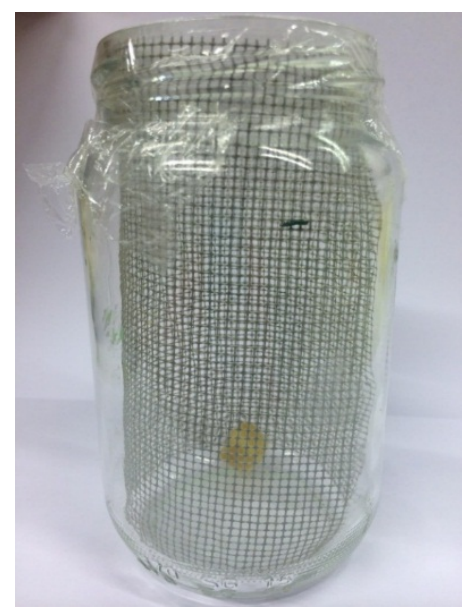

Figure S4. Experimental set-up for measuring pre-death climbing behaviour. Larvae were placed individually in glass jars (120 mm high and $71 \mathrm{~mm}$ in diameter). Sterile mesh wire was placed in the jars to facilitate climbing and a piece of artificial food was placed at the bottom of the jar. 


\section{Chapter}

4

Timely trigger of caterpillar zombie behaviour: temporal requirements for light in baculovirus-induced tree-top disease

\section{This chapter has been published as}

Han Y, van Houte S, van Oers M and Ros VID, 2017. Timely trigger of caterpillar zombie behaviour: Temporal requirements for light in baculovirus-induced tree-top disease. Parasitology 45, 822-827. 


\begin{abstract}
Host behavioural manipulation is a common strategy used by parasites to enhance their survival and/or transmission. Baculoviruses induce hyperactivity and tree-top disease (pre-death climbing behaviour) in their caterpillar hosts. However, little is known about the underlying mechanisms of this behavioural manipulation. Previous study showed that the baculovirus Spodoptera exigua multiple nucleopolyhedrovirus (SeMNPV) induced tree-top disease at 3 days post infection in third instar S. exigua larvae and that light plays a key role in triggering this behaviour. Here we investigated the temporal requirements for the presence of light to trigger this behaviour and found that light from above was needed between 43-50 hours post infection to induce tree-top disease. Infected larvae that were not exposed to light from above in this period finally died at low positions. Exposure to light prior to this period did not affect the final positions where larvae died. Overall we conclude that light in a particular time frame is needed to trigger SeMNPV-induced tree-top disease in S. exigua larvae.
\end{abstract}




\section{Introduction}

The complex interplay between host and invading parasites may result in a wide range of changes both in the host and in the parasite (Lefèvre et al. 2009; Libersat et al. 2009; Hughes 2013; van Houte et al. 2013). Some of these changes are adaptive to the host, e.g. when these changes prevent further parasite dissemination to relatives (Bos et al. 2012). However, some of the alterations appear adaptive to the parasite, thereby enhancing parasite transmission (Lefèvre et al. 2009; van Houte et al. 2013). Parasites, including viruses, may alter host physiology or morphology, but may also manipulate host behaviour (Lefèvre et al. 2009; van Houte et al. 2013). Parasitic manipulation of host behaviour can range from temporal changes of existing behavioural traits to the induction of completely new traits. Cases of behavioural manipulation include changes in host locomotion, reproductive behaviour, feeding and phototactic behaviour (Lefèvre et al. 2009; van Houte et al. 2013).

Baculoviruses are arthropod-specific viruses, mainly infecting lepidopteran larvae (Williams et al. 2016). These viruses are known to induce two behavioural changes in their caterpillar hosts: hyperactivity and tree-top disease (Kamita et al. 2005; Hoover et al. 2011; Katsuma et al. 2012; van Houte et al. 2012; Han et al. 2015; Ros et al. 2015). After infection, caterpillars become hyperactive and prior to death, they climb to the upper parts of plants, where they die. Because baculoviruses are able to liquefy their hosts, death at elevated positions potentially aids the virus to be spread over a larger area of plant foliage, thus increasing virus transmission to subsequent generations of caterpillars (Goulson, 1997; Hoover et al. 2011; Han et al. 2015). Moreover, the exposed caterpillar cadavers are more visible to birds, which feed on these caterpillars and can transport the viruses over large distances (Goulson, 1997).

Though baculovirus-induced behavioural changes were first reported in the late $19^{\text {th }}$ century, it is only during the last decade that the underlying mechanisms have started to be unravelled. Hoover et al. (2011) showed that the ecdysteroid uridine 5'-diphosphate UDPglucosyltransferase (egt) gene of Lymantria dispar MNPV (LdMNPV) is involved in tree-top disease in L. dispar larvae. However, the egt gene of AcMNPV is not involved in inducing treetop disease in S. exigua and Trichoplusia ni larvae: larvae infected with a mutant AcMNPV lacking the egt gene still died at elevated positions (Ros et al. 2015). In these latter two host species, moulting-related climbing (climbing prior to moulting) was affected by egt, but not tree-top disease (climbing prior to death) (Ros et al. 2015). In a different virus-host combination, concerning the baculovirus S. exigua MNPV (SeMNPV) and its single host $S$. exigua, it was 
found that the egt gene might be involved in tree-top disease indirectly, through prolonging the lifespan of infected larvae (Han et al. 2015). Wildtype (WT) SeMNPV-infected third instars climbed to and died at elevated positions between 57 and 67 hours post infection (hpi). Though the larvae infected with a mutant virus (lacking the egt gene) died at lower positions, meanwhile they also had a shorter lifespan (most died before $57 \mathrm{hpi}$ ). Consequently, mutant virus-infected larvae did not reach the time point at which climbing behaviour was observed in WT-infected larvae. Therefore, it is concluded that SeMNPV egt facilitates tree-top disease in S. exigua larvae by extending the larval lifespan (Han et al. 2015). The aforementioned studies showed that the effect of egt on tree-top disease is not a conserved trait among all baculoviruses, and that egt might influence larval time to death or moulting-related climbing behaviour, and therewith it can in some cases (indirectly) affect the outcome of tree-top disease.

Recently, it was found that light plays a key role in the induction of tree-top disease (van Houte et al. 2014; van Houte et al. 2015). Prior to death, S. exigua larvae infected with WT SeMNPV became positively phototactic and showed a strong tendency to move towards light. Infected larvae died at elevated positions when light was given from above, however, larvae died at low positions when the light was provided from below, or when larvae were continuously kept in the dark after infection. Uninfected larvae did not show phototactic behaviour, since larvae kept either in dark or in light conditions behaved similarly (van Houte et al. 2014).

To better understand the role of light in baculovirus-induced behavioural changes, we investigated the importance of the timing of light exposure in the induction of positive phototaxis in SeMNPV-infected S. exigua larvae. In this paper, we show that exposure of WT virus-infected larvae to light between 43 and 50 hpi is important for the induction of lightdependent tree-top disease. In contrast, exposure to light prior to or after this period does not affect the vertical position of the larvae at death.

\section{Material and Methods}

\section{Insect larvae and virus}

Spodoptera exigua larvae were fed on artificial diet and kept at $27^{\circ} \mathrm{C}$ with $50 \%$ relative humidity as described before (Smits et al. 1986) using a 14 L : $10 \mathrm{D}$ photoperiod (7:00 lights on, 21:00 lights off). SeMNPV G25, a naturally occurring WT SeMNPV strain (Murillo et al. 2006), was used in this study. Viral occlusion bodies (OBs) were amplified by infecting 
S. exigua fourth instars and OBs were purified from dead larvae and counted using a BürkerTürk haemocytometer as described before (van Houte et al. 2012).

\section{Behavioural assays}

\section{$\underline{\text { Experimental design }}$}

Three different behavioural assays were performed (see below) and each behavioural assay was executed twice as two independent replicates. For all three assays, newly moulted third instars of S. exigua were infected with WT SeMNPV, using droplet feeding as described before (Han et al. 2015). A viral titre of $10^{6} \mathrm{OBs} / \mathrm{ml}$ was used for infection, which is known to kill at least $90 \%$ of infected larvae. For each treatment, thirty larvae were infected by droplet feeding. As controls, ten mock-infected larvae, droplet fed with a virus-free solution, were used per assay. These mock-infected larvae were included to check for possible contaminations - in all assays mock-infected larvae developed normally and none of these larvae died due to a virus infection. Droplet-fed larvae were placed individually in glass jars (120 mm high and $71 \mathrm{~mm}$ in diameter). Jars contained a cube of artificial diet (approx. $3.5 \mathrm{~cm}^{3}$ ) at the bottom and were lined with sterile mesh wire to facilitate larval climbing. Jars were covered with transparent plastic Saran wrap containing three small holes for ventilation. Jars were incubated at $27^{\circ} \mathrm{C}$ with $50 \%$ relative humidity. The vertical position where the infected larvae died was recorded at five days post infection. Larvae that did not die following virus infection (survived despite being droplet fed with virus or died of other causes) were excluded from analyses (14 out of 840 infected larvae from three behavioural assays).

\section{Behavioural assay 1: light from above, from light to dark conditions}

To determine the time point at which light was needed to trigger positive phototaxis, groups of thirty larvae were switched from normal day/night intervals to dark conditions at different time points post infection. In this assay, if light was used, it was applied from above using three luminescent tubes (18 W each), which were placed $30 \mathrm{~cm}$ above the jars containing the larvae. The side and bottom of the jars were covered with aluminium foil and the jars were placed in a black box to block light from other directions than from above. Five different experimental treatments were used: larvae of group 1 (Gr 1, Figure 1A) were kept in the dark (0 L : $24 \mathrm{D})$ throughout the experiment; larvae of group 2, 3, and 4 (Gr 2, 3, 4 in Figure 1A) were first exposed to the normal 14 L : $10 \mathrm{D}$ photoperiod regime until 43, 50, and 57 hpi, respectively, after which they were switched to completely darkness (0 L : $24 \mathrm{D})$; larvae of the control group 
were kept under normal light/dark conditions (14 L : 10 D) throughout the experiment, using light from above $\left(\mathrm{C}_{\mathrm{a}}\right.$, Figure $\left.1 \mathrm{~A}\right)$.

Behavioural assay 2: light from below, from light to dark conditions

To determine whether the direction of light was important during the time period determined in assay 1, the experiment was repeated with light applied only from below. To this end, three luminescent tubes (18 W each) were placed $30 \mathrm{~cm}$ below the jars containing infected larvae. The side of jars were covered with aluminium foil. A black box was placed over the jars to block light from other directions. Six different experimental conditions were used: larvae of Gr 1-4 (Figure 1A) were applied with the same L : D photoperiods as described in behavioural assay 1, only the light was applied from below instead of above; larvae of two control groups were kept under normal light/dark conditions (14 L : $10 \mathrm{D})$ throughout the experiment using light from above $\left(C_{a}\right.$, Figure 1A) or from below $\left(C_{b}\right.$, Figure $\left.1 A\right)$.

Behavioural assay 3: light from above, from dark to light conditions

In the third behavioural assay we aimed to determine whether light at the beginning of the infection is needed for SeMNPV-induced tree-top disease. Larvae were first kept under complete dark conditions, after which they were switched to a normal light/dark regime. In this assay light was applied from above as described in behavioural assay 1. Three different experimental conditions were used: larvae of Gr 1 (Figure 2A) were kept in the dark (0 L : 24

D) throughout the experiment; larvae of Gr 2 (Figure 2A) were first kept in the dark (0 L : 24

D) until $43 \mathrm{hpi}$, after this point they were switched to normal light/dark conditions (14 L : 10 D); larvae of $\mathrm{C}_{\mathrm{a}}$ (Figure 2A) were kept under normal light/dark conditions (14 L : $10 \mathrm{D}$ ) throughout the experiment.

\section{Data analysis}

The linear regression model ( $\mathrm{lm}$ ) analysis in the program R v3.0.0. (R Core Team, 2013) was used to analyse the position of the larvae at death (Ros et al. 2015). Treatment (different light/dark regime) and experiment (two replicates) were used as explanatory factors and it was determined whether these factors affected the vertical positions of the larvae at death. Since most larvae died as third instar (or during moulting from third to fourth instar), larval stage was excluded as a factor. 


\section{Results}

\section{Light between 43 and 50 hpi triggers SeMNPV-induced tree-top disease}

To investigate during which time period after infection light was needed for SeMNPV-induced tree-top disease, we performed a behavioural assay using virus-infected larvae exposed to different light : dark ( $\mathrm{L}: \mathrm{D})$ regimes (using light from above). Results showed that light between 43 (7:00 hrs at day 2 post infection) and 50 hpi (14:00 hrs at day 2 post infection) was essential to trigger tree-top disease and light after 50 hpi was not needed for tree-top disease. Larvae kept under complete dark conditions from the start of the experiment ( $\mathrm{Gr} 1$ in Figure 1B), or following the 33 hpi point (21:00 hrs at day 1 post infection) (Gr 2 in Figure 1B) died at low positions. However, larvae kept under a 14 L : $10 \mathrm{D}$ photoperiod until 50 hpi (so with light from 43-50 hpi) and then switched to darkness, died at high positions (Gr 3 in Figure 1B) (Gr 1 (n $=58)$ and $\operatorname{Gr} 2(n=59)$ versus $\operatorname{Gr} 3(n=59) ; T$-test $=3.174$ and 4.013, respectively; d.f. $=288$; $p<0.01$ and $p<0.001$, respectively). Moving larvae to complete dark conditions at a later time point (57 hpi, Gr 4) or keeping them under normal L : D conditions $\left(\mathrm{C}_{\mathrm{a}}\right)$ throughout the experiment did not affect the larval position at death: larvae of these treatments also died at high positions (Figure 1B) ( Gr $3(n=59)$ versus $\mathrm{Gr} 4(n=58)$ and $\mathrm{Gr} \mathrm{C}_{\mathrm{a}}(n=60) ; T$-test $=0.738$ and 1.276, respectively; d.f. $=288 ; p=0.461$ and 0.203 , respectively). There was no significant difference between the two replicates $(T$-test $=1.039$; d.f. $=288 ; p=0.300)$. We conclude that light between 43 and 50 hpi was important to trigger SeMNPV-induced tree-top disease and light after 50 hpi did not have a measurable influence on the outcome of tree-top disease.

\section{The direction of light is important for tree-top disease}

To determine whether the direction of light was important during the time period determined in assay 1, the behavioural assay was repeated with light applied from below. Two control groups, kept under a normal light: dark regime (14 L : 10 D), were included, one using light from above $\left(\mathrm{C}_{\mathrm{a}}\right)$ and one using light from below $\left(\mathrm{C}_{\mathrm{b}}\right)$. Larvae of control group $\mathrm{C}_{\mathrm{a}}$ died at high positions (Figure 1C) as expected, indicating that infected larvae still reacted to light in this experiment. Larvae of all other treatments died at low positions ( $\mathrm{Gr} 1$ to 4 and $\mathrm{C}_{\mathrm{b}}$ in Figure $1 \mathrm{C}$ ), also when receiving light from below during the period determined in assay 1 as being crucial for the induction of tree-top disease when light was applied from above (all differences when making comparisons between $\mathrm{Gr} 1-4$ and $\mathrm{C}_{\mathrm{b}}$ are non-significant $(T$-test $<1.7$ and $p>0.08$ for all comparisons; d.f. $=348$, Table $\mathrm{S} 1)$; $\mathrm{Gr} \mathrm{C}_{\mathrm{a}}$ is significantly different from $\mathrm{Gr} 1-4$ and $\mathrm{C}_{\mathrm{b}}(T-$ test $>5.9$ and $p<0.001$ for all comparisons; d.f. $=348$, Table S1). This finding indicates that 
the direction of light during this period (43 to $50 \mathrm{hpi}$ ), is crucial and tree-top disease is only observed if light is applied from above during 43 to $50 \mathrm{hpi}$. The two replicates of this experiment were not significantly different from each other $(T$-test $=0.927$; d.f. $=348 ; p=0.355)$.
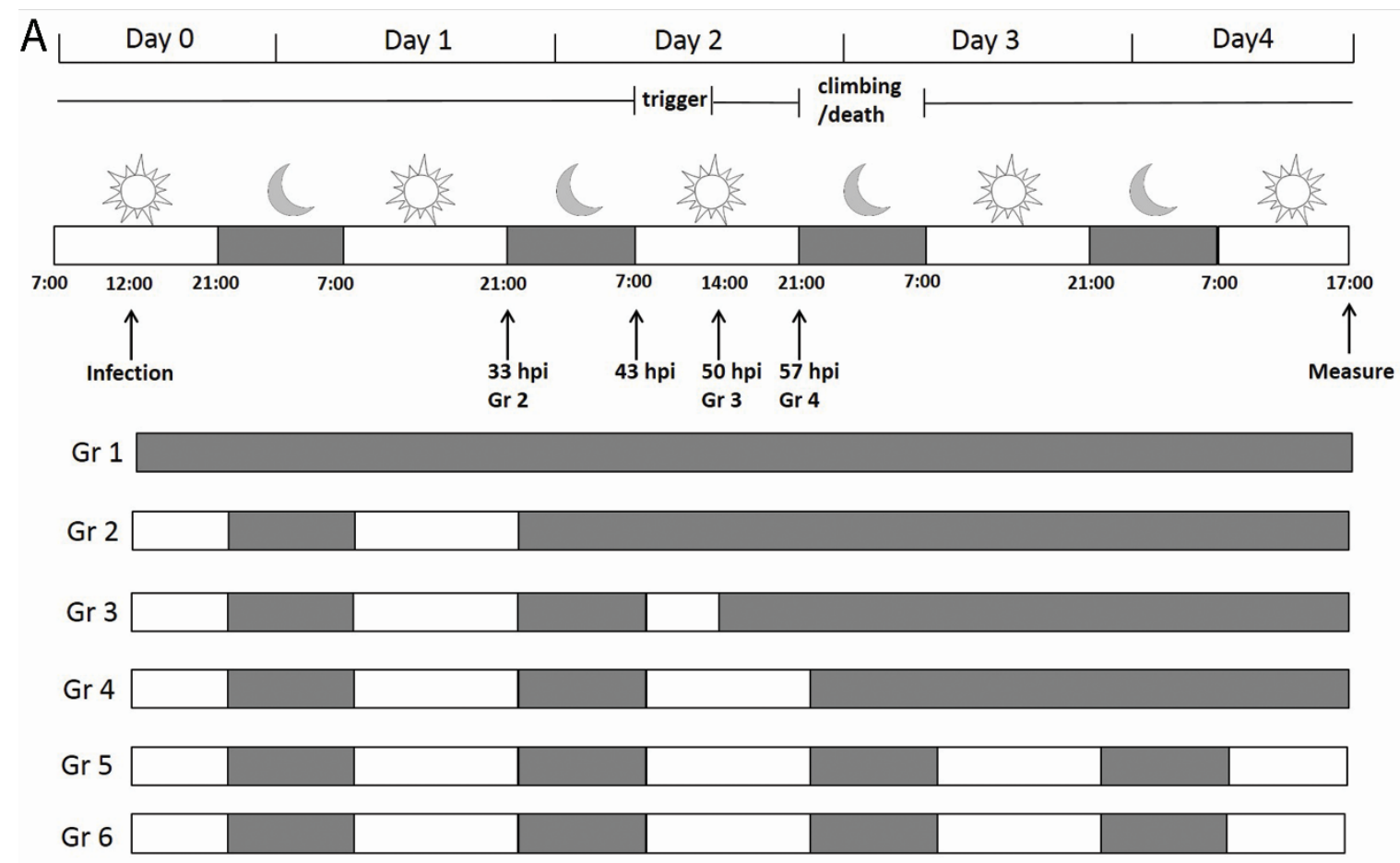

B

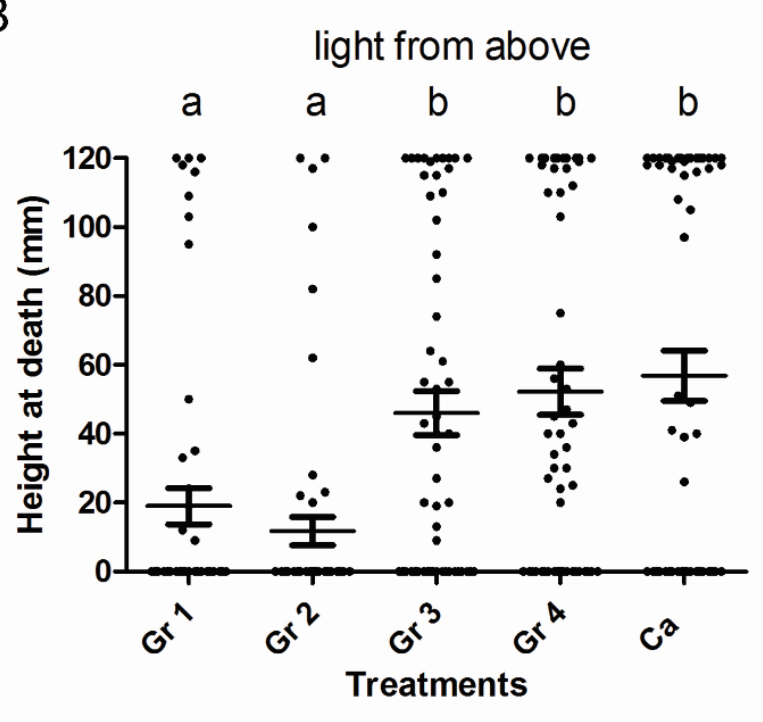

C

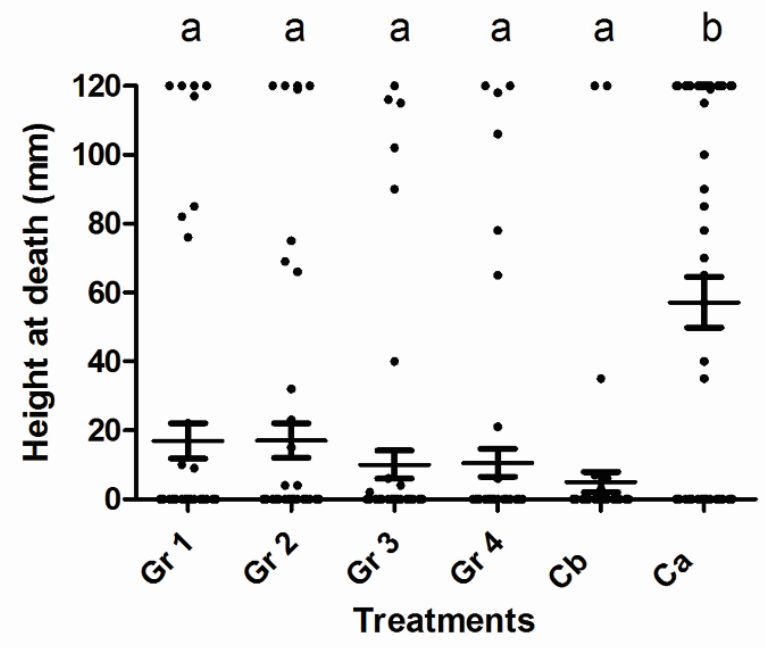

Figure 1. Light between 43 and $50 \mathrm{hpi}$ is needed to trigger SeMNPV-induced phototaxis in Spodoptera exigua larvae. (A) Scheme of the experimental set-up with grey representing a dark interval and white representing a light interval. Vertical arrows indicate the time points at which the infection was done, the different treatment groups (Gr 2-4) were moved to continuous dark conditions and the measurement of the final vertical position of larvae was done. For each treatment $\left(\mathrm{Gr} 1-4, \mathrm{C}_{\mathrm{a}}, \mathrm{C}_{\mathrm{b}}\right)$, the dark - light scheme is indicated. $\mathrm{C}_{\mathrm{a}}$ represents a control with light from above that was included in both behavioural assays. $\mathrm{C}_{\mathrm{b}}$ represents a control with light from below that was included only in behavioural 
assay 2. The period during which the phototaxis was triggered ('trigger') and the period during which the larvae climbed to elevated positions and subsequently died ('climbing/death') are indicated. (B) Height at death of larvae of behavioural assay 1 (light provided from above; Gr 1 ( $\mathrm{n}=58)$, Gr 2 ( $\mathrm{n}=59$ ), Gr 3 ( $\left.n=59), \operatorname{Gr} 4(\mathrm{n}=58), \mathrm{C}_{\mathrm{a}}(\mathrm{n}=60)\right)$. (C) Height at death of larvae of behavioural assay 2 (light provided from below; Gr $1(n=59)$, Gr $2(n=60)$, Gr $3(n=59)$, Gr $\left.4(n=60), C_{b}(n=58), C_{a}(n=59)\right)$. Data points represent the height at death $(\mathrm{mm})$ of individual larvae. Horizontal lines show the mean value of height at death and whiskers the standard error of the mean. Treatment groups marked with a different letter (a or b) are significantly different $(\mathrm{P}>0.05)$.

\section{Light between 0 and 43 hpi is not needed for tree-top disease}

We also studied whether additional light exposure between 0 and 43 hpi was needed to trigger tree-top disease. Therefore, infected larvae were first kept in darkness until 43 hpi, after which light was applied from above following a $14 \mathrm{~L}: 10 \mathrm{D}$ period (Gr 2, Figure 2). Data showed that infected larvae exposed to these conditions (Gr 2, Figure 2B) died at high positions compared to larvae kept in completely dark conditions throughout the experiment (Gr 1, Figure 2B; dying at low positions; Gr 2 ( $\mathrm{n}=59)$ versus $\mathrm{Gr} 1(\mathrm{n}=60)$; T-test $=-2.587$; d.f. $=175 ; p<0.01)$. Moreover, infected larvae exposed to above mentioned conditions ( $\mathrm{Gr} 2$, Figure 2B) died at similar positions compared to larvae kept under a $14 \mathrm{~L}: 10 \mathrm{D}$ light regime throughout the experiment ( $\mathrm{Gr} \mathrm{C}_{\mathrm{a}}$, Figure 2B and 2C; dying at elevated positions; Gr $2(\mathrm{n}=59)$ versus $\mathrm{C}_{\mathrm{a}}(\mathrm{n}=60)$; $T$-test $=1.685 ;$ d.f. $=175 ; p=0.973)$. The two replicates were not significantly different $(T$-test $=0.084$; d.f. $=175 ; p=0.933)$. The experimental data obtained in the third behavioural assay further suggest that a light stimulus from above is needed during the period from 43 to 50 hpi to successfully induce tree-top disease, and that light prior to this period does not have a measurable influence on the outcome of tree-top disease. 

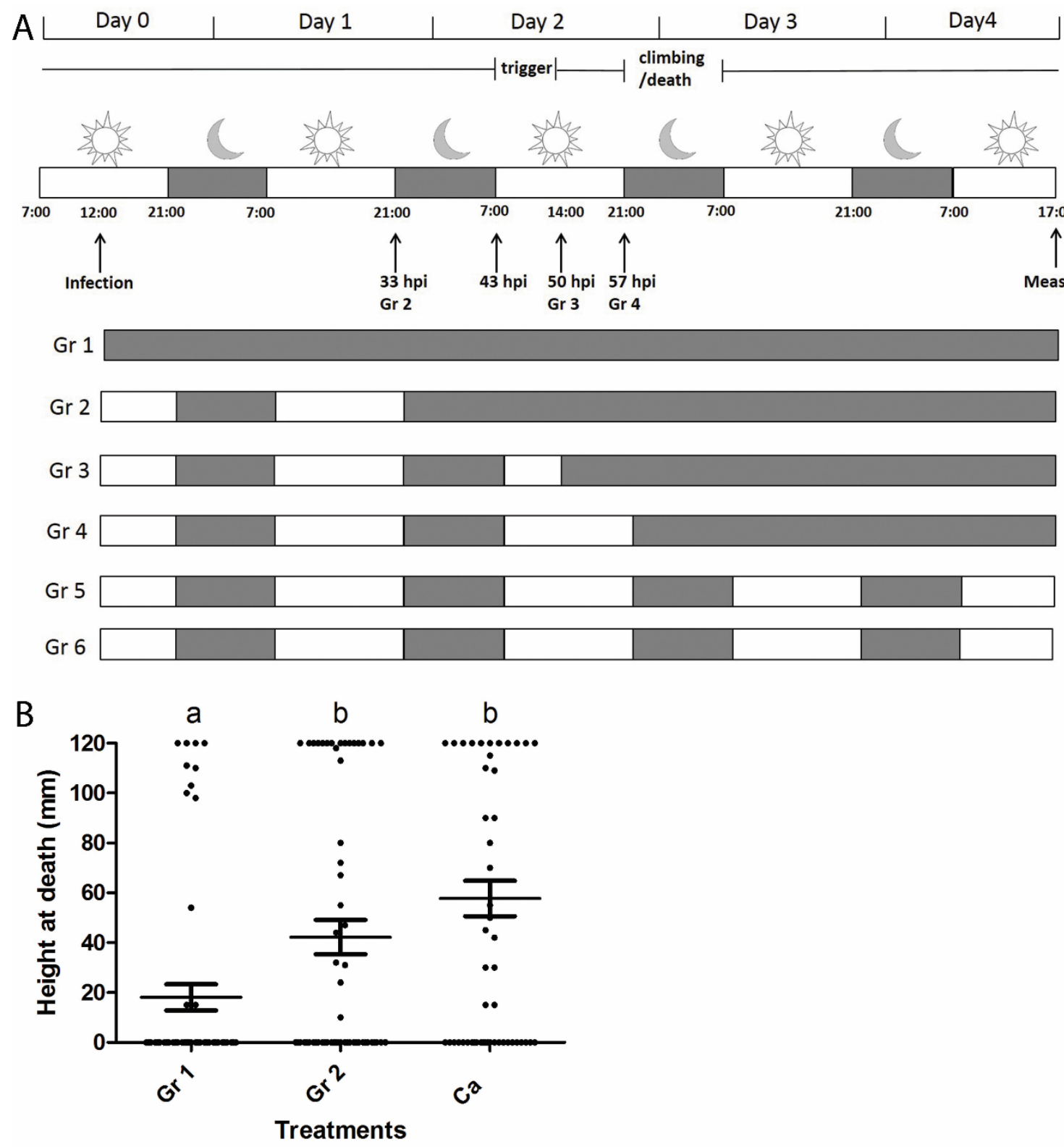

Figure 2. Light exposure between 0 and 43 hours post infection does not affect tree-top disease.

(A) Scheme of the experimental set-up with grey representing a dark interval and white representing a light interval. Vertical arrows indicate the time points at which the infection was done, treatment group Gr 2 was moved from continuous dark conditions to a normal L : D rhythm and the measurement of the final vertical position of larvae was done. For each treatment ( $\mathrm{Gr} 1, \mathrm{Gr} 2, \mathrm{C}_{\mathrm{a}}$ ) the dark - light scheme is indicated. $\mathrm{C}_{\mathrm{a}}$ represents a control with light from above. The period during which the phototaxis was triggered ('trigger') and the period during which the larvae climbed to elevated positions and subsequently died ('climbing/death') are indicated. (B) Height at death of larvae of behavioural assay 3 [light provided from above; Gr $1(n=60), \operatorname{Gr} 2(n=59), C_{a}(n=60)$ ]. Data points represent the height at death $(\mathrm{mm})$ of individual larvae. Horizontal lines show the mean value of height at death and whiskers the standard error of the mean. Treatment groups marked with a different letter ( $a$ or b) are significantly different $(\mathrm{P}>0.05)$. 


\section{Discussion}

Baculovirus-induced behavioural changes have important ecological and evolutionary consequences for both the host and the pathogen. Exciting progress has been made to reveal the underlying mechanisms. Previously, it has been shown that light applied from above is needed for the induction of tree-top disease by the baculovirus SeMNPV in S. exigua larvae (van Houte et al. 2014; van Houte et al. 2015). Here, we further investigated the role of light in this process and found that for SeMNPV-infected third instars light from above is needed between 43 and 50 hpi to trigger tree-top disease. The positive phototactic behaviour displayed prior to death was triggered in this specific time span.

Strikingly, light from above was needed to induce tree-top disease between 43 and 50 hpi, but not during the period when the actual climbing took place. When light was provided from above between 43 and $50 \mathrm{hpi}$, WT-infected larvae climbed to and died at elevated positions between 57 and $67 \mathrm{hpi}$, even though light was absent during the period in which they climbed. Apparently, positive phototaxis was already triggered between 43 and $50 \mathrm{hpi}$, so prior to the actual climbing. Molecular pathways that lead to positive phototaxis might be activated in the infected larvae during this period. Once the pathways for positive phototaxis are activated, infected larvae do not need light anymore to climb to elevated positions. When light was provided from below in the same time span (43 to $50 \mathrm{hpi}$ ) larvae stayed at the bottom until death (behavioural assay 2). In both experiments the infected larvae moved towards the direction where the light came from in the induction period (between 43 and $50 \mathrm{hpi}$ ), though during climbing (57 to $67 \mathrm{hpi}$ ) they were in the dark. An alternative explanation is that the larvae somehow 'remember' the direction of the light (present during the trigger period) when they are climbing (during the night when light is absent).

Previous studies showed that the egt gene from SeMNPV is involved in SeMNPV induced treetop disease in S. exigua indirectly: most WT-infected larvae climbed to and died at elevated positions between 57 and 67 hpi when light was provided above. The current experiments suggest that before the actual climbing, pathways for positive phototaxis have been activated. However, larvae infected with the SeMNPV virus lacking the egt gene have been shown to start dying from $43 \mathrm{hpi}$ and most of these larvae already died before 57 hpi before actual climbing started in WT-infected larvae. Due to this earlier death, larvae infected with the egt-minus virus did not reach the point of climbing, although the pathways for positive phototaxis might have been activated also in the egt-minus virus infected larvae (Han et al. 2015; Ros et al. 2015). 
Though phototaxis has been observed and studied in many insect species, the underlying mechanisms are still not completely understood. In general, insects sense light of certain wavelengths using their photoreceptors (Castrejon and Rojas, 2010; Yamaguchi and Heisenberg, 2011; Otsuna et al. 2014; Sun et al. 2014;). Neuron cells can sense the output from photoreceptors and deliver the signal to the insect's central nervous system (CNS). In the CNS, different pathways might be triggered that finally lead to the phototactic behaviour. However, the trigger appears to differ among different insect species. For example, lepidopteran larvae and moths show a strong preference for green and blue light (520 and $460 \mathrm{~nm}$ in wavelength) (Castrejon and Rojas, 2010; Sun et al. 2014), while the fruit fly Drosophila melanogaster prefers light with shorter wavelength, like ultraviolet (UV) light (400 nm in wavelength) (Fischbach, 1979; Otsuna et al. 2014). Honey bees (Apis mellifera) have three spectral types of photoreceptors, for UV, blue and green light, while D. melanogaster has five types of photoreceptors differing in spectral properties (Yamaguchi and Heisenberg, 2011). A few downstream genes have been identified to play a role in phototaxis. For example, the tim and per genes, which encode components of the circadian clock, are important for phototactic behaviour in D. melanogaster larvae (Keene and Sprecher, 2012). The neurotransmitter serotonin was found to play a role in phototactic behaviour in honey bees (Yamaguchi and Heisenberg, 2011).

Positive phototaxis is not only induced in baculovirus-infected caterpillars, but also in other parasite-host systems. Parasites may induce positive phototaxis by invading or affecting the CNS of their hosts. Crickets infected with Gordian worms present strong phototaxis shortly before the maturation of the Gordian worms. Moreover, the phototaxis is reversible: once the mature Gordian worms are released, the crickets are not attracted to light anymore (Ponton et al. 2011). A comparative proteomic study revealed that manipulated crickets exhibit higher expression levels of proteins involved in vision (CRAL_TRIO), CNS development, neurogenesis, circadian rhythm and neurotransmitter production (Biron et al. 2006). Positive phototaxis is also observed in amphipods infected with trematodes or acanthocephalans (both parasitic worms), which stimulate the amphipods (the intermediate host of the parasitic worms) to move closer to the water surface, where they can be consumed by predators (forming the subsequent hosts). In the gammarid Gammarus insensibilis infected with the trematode Microphallus papillorobustus expression levels of proteins that are involved in serotonin synthesis (aromatic-L-amino acid decarboxylase) and vision (CRAL_TRIO) are significantly higher than in non-infected G. insensibilis. It has been shown that in many invertebrates 
phototactic behaviour is related with serotonin synthesis alteration (Ponton et al. 2006). Likewise, in the gammarid Gammarus pulex infected with acanthocephalan parasites, serotonin levels are also changed and have been functionally linked to changed behaviour upon light perception (Tain et al. 2006). The freshwater amphipod Hyalella azteca infected with the acanthocephalan Corynosoma constrictum showed a significantly higher response to green light $(500-550 \mathrm{~nm})$ and red light $(600-700 \mathrm{~nm})$, but the response to blue light (400-450 nm) was not changed (Benesh et al. 2005). In Dolichoderus thoracicus ants infected with the fungus Ophiocordyceps pseudolloydii and in Succinea putris snails infected with the parasitic flatworm Leucochloridium paradoxum, the infected hosts display positive phototactic behaviour (Wesołowska and Wesołowski, 2014; Chung et al. 2017), however, the underlying mechanisms are still unclear. Though in the described examples the individual parasites are not phylogenetically related (representing worms, viruses or fungi), they may make use of similar proximate mechanisms to modify light perception or the response there to in their hosts. We hypothesize that SeMNPV hijacks host light perception pathways in the central nervous system (CNS) to induce tree-top disease in S. exigua and that timing of light perception plays a key role in this process. It remains to be elucidated which spectrum of the light is needed during this period. It is noticeable that light also plays a role in Bombyx mori nucleopolyhedrovirus (BmNPV)-induced hyperactivity in B. mori larvae. Light did not induce positive phototaxis in infected larvae, since both virus- and mock-infected larvae showed similar levels of phototaxis. However, light enhanced the amplitude of BmNPV-induced hyperactivity; when light was present, the induced hyperactivity was more than two fold higher than under dark conditions (Kamita et al. 2005).

Overall we conclude that light perception is required between 43 and 50 hpi to trigger SeMNPV-induced tree-top disease in third instar S. exigua larvae. Pathways leading to positive phototaxis might be activated during this period, which leads to movement in the direction of the earlier provided light at a later stage of the infection.

\section{Acknowledgements}

We thank Els Roode and Hanke Bloksma for their help in rearing the larvae. We thank Amaya Serrano for providing the SeMNPV G25 isolate and Jan van Lent for useful discussions throughout the experiments. 


\section{Chapter}

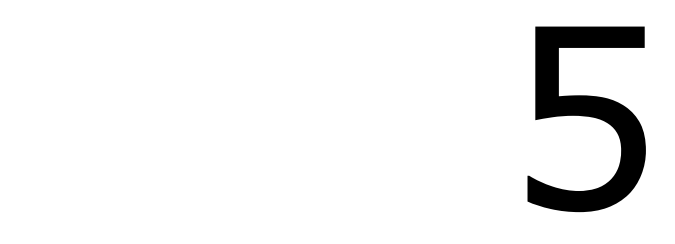

Baculovirus PTP2 functions as a pro-apoptotic protein

\section{This chapter has been published as}

Han Y, van Houte S, van Oers M, Ros VID, 2018. Baculovirus PTP2 functions as a proapoptotic protein. Viruses 10, 181. 


\begin{abstract}
The family Baculoviridae encompasses a large number of invertebrate viruses, mainly infecting caterpillars of the order Lepidoptera. The baculovirus Spodoptera exigua multiple nucleopolyhedrovirus (SeMNPV) induces physiological and behavioral changes in its host Spodoptera exigua, as well as immunological responses, which may affect virus transmission. Here we show that the SeMNPV-encoded protein tyrosine phosphatase 2 (PTP2) induces mild apoptosis in Spodoptera frugiperda (Sf) 21 cells upon transient expression. Transient expression of a catalytic-site mutant of ptp2 did not lead to apoptosis, indicating that the phosphatase activity of PTP2 is needed to induce apoptosis. We also found that the caspase level (indicator of apoptosis) was higher in cells transfected with the $p t p 2$ gene than in cells transfected with the catalytic mutant. Adding a caspase inhibitor reduced the level of ptp2induced apoptosis. Moreover, deletion of the ptp2 gene from the viral genome prevented the induction of apoptosis in S. exigua hemocytes. The virus titer and virulence indices (the viral infectivity and the time to death) were not affected by deletion of the $p t p 2$ gene. However, the viral occlusion body yield from S. exigua larvae infected with the mutant virus lacking the $p t p 2$ gene was much lower than the yield from larvae infected with the wild-type (WT) virus. We hypothesize that the observed pro-apoptotic effects of PTP2 are the result of PTP2-mediated immune suppression in larvae, which consequently leads to higher viral occlusion body yields.
\end{abstract}




\section{Introduction}

Apoptosis is an active process of programmed cell death that is involved in immunity, normal development and cell differentiation (Elmore, 2007). It is characterized by morphological changes, including cell blebbing and cell shrinkage, chromosomal DNA fragmentation, as well as energy-dependent biochemical changes (Elmore, 2007; Feng et al., 2007). Apoptosis is part of the insect's innate immune response against pathogens, including viruses, triggering premature cell death of infected host cells (Kvansakul, 2017). Viruses have developed a range of strategies to counteract host-induced apoptosis (Kvansakul, 2017), including the expression of anti-apoptotic genes. On the other hand, some viruses have been found to actively induce apoptosis in their host in order to suppress the host immune system and/or enhance virus dissemination (Chitnis et al., 2011; Suderman et al., 2008). For example, it was found that the polydnavirus Microplitis demolitor bracovirus (MdBV) induced apoptosis in immune cells of Spodoptera frugiperda caterpillars and that this activity contributed to the immunosuppression of the host (Suderman et al., 2008). Several viral genes and proteins have been found to induce pro-apoptotic effects in host-derived cell lines (Anasir et al., 2017; Chitnis et al., 2011; SchultzCherry et al., 2001; Schultz et al., 2009; Suderman et al., 2008). For example, Suderman et al. (Suderman et al., 2008) found that the protein tyrosine phosphatase-H2 (PTP-H2) from MdBV induced apoptosis in S. frugiperda 21 (Sf21) cells and showed that the phosphatase activity of PTP-H2 was needed for the apoptotic activity. The iridovirus serine/threonine kinase (ISTK) from Chilo iridescent virus (CIV) induced apoptosis in cell lines derived from the spruce budworm Choristoneura fumiferana and the boll weevil Anthonomus grandis (Chitnis et al., 2011).

Baculoviruses are arthropod-specific viruses with a circular, double-stranded DNA genome (Williams et al., 2017). Two types of baculovirus virions are present in a single infection cycle: occlusion-derived virions (ODVs) and budded virions (BVs). ODVs are embedded in occlusion bodies (OBs) and are responsible for starting the primary infection in epithelial midgut cells and for host-to-host transmission, while BVs are responsible for spreading viruses throughout the body via the hemolymph (Clem and Passarelli, 2013; Williams et al., 2017). Baculoviruses infect larval stages of insects, mainly of the order Lepidoptera. The baculovirus Spodoptera exigua multiple nucleopolyhedrovirus (SeMNPV) is highly infectious to its single host Spodoptera exigua, the beet armyworm, and is an important biocontrol agent of this species (Smits et al., 1986). Apoptosis is observed in host insects infected by baculoviruses (Feng et al., 2007; Wan et al., 2015). SeMNPV infection has been suggested to trigger apoptosis in the 
hemocytes of S. exigua larvae (Wan et al., 2015). In addition, in the hemolymph and fat bodies of $S$. exigua larvae apoptosis was observed following infection by Spodoptera litura nucleopolyhedrovirus (SpltNPV) (Wan et al., 2015). Baculoviruses contain pro-apoptotic genes, for example, the immediate early gene 1 (iel) from the baculovirus Autographa californica multiple nucleopolyhedrovirus (AcMNPV). Previous studies have shown that the iel gene from AcMNPV is involved in the formation of apoptotic bodies in virus-infected Sf 21 cells by initiating virus DNA replication events that subsequently trigger cell death (Prikhod'ko and Miller, 1996; Schultz and Friesen, 2009; Schultz et al., 2009). Apart from pro-apoptotic genes (like iel), baculoviruses also contain anti-apoptotic genes. AcMNPV carries the anti-apoptotic gene $p 35$, while other baculoviruses contain either $p 35$ homologues or inhibitor of apoptosis (iap) genes (Clem, 2001). SeMNPV possesses iap2 and iap3 homologues (IJkel et al., 1999). SeMNPV IAP3 was able to block chemical induction of apoptosis in insect cells and mammalian cells; however, the function of iap2 is not known yet (Wu et al., 2016; Zhu et al., 2008).

SeMNPV, like bracoviruses, carries a phosphatase gene, in this case called protein tyrosine phosphatase 2 (ptp2). Both SeMNPV PTP2 and MdBV PTP-H2 contain a His-Cys (HC) signature motif in their catalytic site, which is characteristic for proteins belonging to the PTP superfamily. Moreover, the PTP2 protein carries a C-terminal consensus sequence that is characteristic for mitogen-activated protein kinase (MAPK) phosphatases. Several MAPK phosphatases have been reported to regulate MAPK pathways and are important regulators of apoptosis (Patterson et al., 2009). Here, we tested whether SeMNPV PTP2 has a similar function to the MdBV PTP-H2 in inducing apoptosis in its host. First, we investigated the apoptotic effect of PTP2 on cultured cells and on S. exigua larval hemocytes. Then we compared the budded virus titer, the virulence indices (including the viral infectivity and the time to death) and the OB yield between insects infected with either the wild type (WT) or the mutant virus lacking the $p t p 2$ gene. The results show that PTP2 functions as a pro-apoptotic protein in cultured cells and in larval hemocytes. Moreover, PTP2 contributes to a higher OB yield in larvae. 


\section{Materials and Methods}

Insects, cell lines and virus

Spodoptera exigua larvae were reared on artificial diet as described before (Han et al., 2015). Sf21 cells (Sigma-Aldrich) were maintained as monolayers in Grace's medium (Invitrogen) supplied with $10 \%$ fetal bovine serum (FBS) (Invitrogen) and $0.1 \%$ gentamycin $(50 \mu \mathrm{g} / \mathrm{mL}$, Invitrogen). Se301 cells, originally derived from S. exigua (Hara et al., 1995), were maintained as monolayers in CCM3 serum free medium (Hyclone) supplied with 5\% FBS and 0.1\% gentamycin $(50 \mu \mathrm{g} / \mathrm{mL})$. The SeBac10 bacmid, derived from the SeMNPV US-1 strain (Pijlman et al., 2002), was used in this study.

\section{Assessment of apoptosis in Sf21 cells}

\section{$\underline{\text { Construction of plasmids for transient expression assays }}$}

The pIB-DEST expression vector (Invitrogen) was used for transient expression assays in Sf21 cells. The vector contains the constitutively expressed early OpIE2 promoter, derived from Orgyia pseudotsugata (Op) MNPV, to drive the expression of the gene of interest. Each gene to be expressed was cloned downstream of the egfp open reading frame (ORF), from which it was separated by the foot and mouth disease virus $2 \mathrm{~A}$ ribosome skipping element (FMDV2A). EGFP was used to monitor the transfection efficiency. FMDV2A is a seventeen amino acids long (NFDLLKLAGDVESNPGP) element that allows co-translational cleavage between the marker protein (EGFP) and the protein to be analyzed. In total five pIB-DEST constructs were made: (i) modified pIB-DEST vector (pIB-DESTmod) in which the chloramphenicol resistance (cat) gene and the $c c d B$ gene were replaced by a 888 bp long internal DNA segment from the AcMNPV gp64 ORF (nt509-1396) to have a negative control vector that could be amplified in the $E$. coli strain DH5 $\alpha$ (the $c c d B$ gene in the original pIB-DEST is lethal to E. coli); (ii) pIBEGFP encoding only EGFP; (iii) pIB-SePTP2 encoding EGFP and SeMNPV PTP2; (iv) pIBSePTP2mut encoding EGFP and SeMNPV PTP2 containing a mutation in its catalytic site (mutation of cysteine to serine at position 110 (C110S)); and (v) pIB-AcPTP encoding EGFP and the AcMNPV PTP protein (distantly related to PTP2) (van Houte et al., 2012), to allow comparison with a different viral protein tyrosine phosphatase. To construct these plasmids, the AcMNPV gp64 segment and the ptp ORF were amplified from the AcMNPV E2 bacmid (Luckow et al., 1993) using primer pairs 1 and 2, and 3 and 4, respectively (Table S1). The Septp2 ORF was amplified from the SeMNPV bacmid SeBac10 using primers 5 and 6 (Table $\mathrm{S} 1)$. To obtain the C110S catalytic mutant of Septp2 a $p t p 2^{C 110 S}$ mutation was created by a two- 
step PCR reaction using SeBac10 as template. First, primer 7 (Table S1), which introduced two point mutations at nucleotides 328 and 330 relative to the ATG start codon, was combined with primer 8 (Table S1), which annealed to the $3^{\prime}$ end of the ptp2 ORF, to create a $3^{\prime}$ segment with the required mutations. The resulting $198 \mathrm{bp}$ PCR product was used as reverse primer and combined with primer 9 (Table S1), annealing to the $5^{\prime}$ end of the ptp2 ORF, to create the full length $p t p 2^{C 110 S}$ insert, again with $\mathrm{SeBac} 10$ as a template. Then the $p t p 2^{C 110 S}$ insert was verified by sequence analysis after cloning into the pJET1.2 cloning vector (Fermentas). The resulting plasmid pJET-ptp $2^{C 110 S}$ was then used as template for PCR using the primers 5 and 6 to allow Gateway cloning (see below).

The forward primers were designed to introduce an attB1 site and a HindIII site, while the reverse primers introduced an attB2 site (Table S1) to enable Gateway ${ }^{\circledR}$ cloning (Invitrogen). All PCRs were performed with the proofreading Phusion polymerase (Finnzymes). The resulting PCR amplicons were first cloned into the pDONR207 donor plasmid (Invitrogen) and sequenced and subsequently cloned into the pIB-DEST plasmid. The EGFP-FMDV2A was obtained as HindIII fragment from the plasmid CHIKrep-pac2AEGFP as described before (Fros et al., 2010) and inserted into HindIII-linearized pIB-DEST plasmids containing the genes of interest.

\section{Transient expression assay}

In total, two transient expression assays were conducted and each assay was performed twice as two independent replicates. In the first assay the apoptotic effects of the above-mentioned pIB-DEST-derived plasmids on Sf21 cells was assessed. In total, seven treatments were included in this assay: (1) pIB-EGFP + Actinomycin D (ActD, Sigma-Aldrich), a chemical inducer of apoptosis, as a positive control; (2) pIB-SePTP2; (3) pIB-SePTP2mut; (4) pIBAcPTP; (5) pIB-EGFP; (6) pIB-DESTmod, to monitor whether the pIB-DEST vector itself induces apoptosis on Sf21 cells; and (7) mock transfected cells (no vector used). The second transient expression assay was conducted to assess whether apoptosis inhibitors were able to inhibit Septp2 induced apoptosis in Sf21 cells. In this case cells were transfected with pIBSePTP2, in the presence or absence of the pan-caspase inhibitor carbobenzoxy-valyl-alanylaspartyl-(O-methyl)-fluoromethylketone (Z-VAD-FMK, Promega).

For both transfection assays Sf21 cells were seeded at a confluency of $25-30 \%$ in 6-well plates. The cells were incubated overnight and two $h$ prior to transfection, the culture medium was replaced with Grace's medium without serum. Transfections were performed with $4 \mu \mathrm{g}$ of the 
pIB-DEST plasmids containing the genes of interest. Cellfectin II (Invitrogen) was used as the transfection reagent according to the manufacturers' protocol. ActD was added to the medium $40 \mathrm{~h}$ post transfection (hpt) at a final concentration of $0.25 \mu \mathrm{g} / \mathrm{mL}$. The caspase inhibitor ZVAD-FMK was supplied in the normal medium at a concentration of $20 \mu \mathrm{M}$ at $5 \mathrm{hpt}$. Cells were monitored daily using a Zeiss Axio Observer inverted fluorescence microscope to analyze EGFP expression and apoptotic body formation.

\section{Caspase assay}

A caspase assay (treatments) were performed using the experimental set-up for transient expression assay 1 described above; the assay was performed twice as two independent replicates. The transfection and apoptosis induction procedures were the same as described above, with the exception that all transfections were performed in 24-well plates, making each of the reagent volumes four times smaller. At $48 \mathrm{hpt}$ cells were homogeneously resuspended in culture medium. For each sample, $50 \mu \mathrm{L}$ of cell suspension was mixed with $50 \mu \mathrm{L}$ of caspaseglo 3/7 substrate (Promega) in white 96-well plates. All samples were prepared in duplo per replicate. The plate was incubated in the dark for one hour at room temperature. Luminescence was measured in a fluorometer (Optima; settings: 5 flashes with maximal gain and top optic measurement). Luminescence was measured as relative luminescent units (RLUs). Differences in RLUs between the treatments were tested using the software package SPSS, version 22.0 (Corp, 2013). Data were first tested for normality (Kolmogorov-Smirnov test) and homogeneity of group variance (Levene's test). Where possible, logarithmic transformations were performed to attain normality and homogeneity of variances. A one-way analysis of variance (ANOVA) was performed to determine whether there were significant differences in RLUs among the different treatments. When a significant difference was found, pairwise comparisons were performed using Tukey post hoc tests.

\section{Assessment of apoptosis in Spodoptera exigua hemolymph cells}

\section{Construction of recombinant virus}

An SeBac10-derived bacmid with a partial deletion of the ptp2 ORF ( $\Delta p t p 2)$ was constructed using the combined lambda RED and Cre-recombination methods described before (Ros et al., 2015). The mutant had a deletion of the major part (354 bp) of the ptp2 ORF (498 bp), ranging from nucleotide 48 to 401 . Briefly, PCR products of the chloramphenicol (cat) resistance gene flanked by modified loxP sites and with 50 bp overhangs homologous to the flanking regions of the region to be deleted were generated with Phusion polymerase using primer 10 and 11 
(Table S1). Subsequently, the major part of the ptp2 ORF was replaced by the cat gene flanked by modified $\operatorname{lox} P$ sites. The $c a t$ gene was then removed by Cre-recombinase, leaving an inserted segment of $162 \mathrm{bp}$ that contained the recombined loxP site. The deletion of the cat gene was checked by PCR using primers 12 and 13 (Table S1) that annealed to the $5^{\prime}$ and $3^{\prime}$ areas outside the deleted region. To enable oral infection of $S$. exigua larvae, the SeMNPV polyhedrin promoter and ORF were introduced into the $\Delta p t p 2 \mathrm{SeBac10}$, which, similar to the ancestral SeBac10, lacks the polyhedrin gene, as described before (Han et al., 2015).

Recombinant $\Delta p t p 2$ SeMNPV viral OBs were generated by injecting $\Delta p t p 2$ SeBac10 bacmid DNA together with transfection reagent into 4th instar $S$. exigua larvae as described before (Han et al., 2015). $\Delta p t p 2$ SeMNPV OBs were amplified in and purified from larvae using protocols as described before (Han et al., 2015). The concentration of OBs was determined using a Bürker-Türk hemocytometer (Marienfeld). SeBac10 derived WT virus (WT SeMNPV) (Han et al., 2015) was used as a control.

\section{Apoptosis in S. exigua larvae hemocytes}

Late 2 nd instars of $S$. exigua were starved overnight for $16 \mathrm{~h}$ and allowed to molt. The next morning, newly molted 3rd instars were infected with WT SeMNPV or $\Delta p t p 2$ SeMNPV using droplet feeding as described before (Ros et al., 2015). Viral concentrations of $10^{6} \mathrm{OBs} / \mathrm{mL}$, known to kill at least $90 \%$ of WT SeMNPV-infected larvae, were used for infection. A virusfree sucrose solution was used for mock infections. For each treatment, 48 larvae were infected and the experiment was performed twice as two independent replicates.

Apoptosis of hemocytes was measured by bleeding 3rd instar S. exigua larvae from a cut proleg $48 \mathrm{~h}$ post infection (hpi). Two microliter hemolymph was collected from each infected larva and the hemolymph samples from 10 larvae were pooled in $80 \mu \mathrm{L}$ PBS containing 1-phenyl-2thiourea (PTU, $0.1 \%$ final concentration) to prevent melanization. Next, $5 \mu \mathrm{L}$ of Annexin $\mathrm{V}$ EGFP (Annexin V-EGFP Apoptosis Detection Kit, BioVision) was added to stain any apoptotic cells, while Hoechst dye was added at a concentration of $10 \mu \mathrm{M}$ to stain the nuclei of all cells. The mixtures were incubated at RT for 10 minutes and fluorescence was studied with a Zeiss Axio Observer inverted microscope.

\section{$\underline{\text { RNA extraction and RT-PCR }}$}

To confirm the expression of ptp2 in WT SeMNPV-infected larvae, and to confirm the absence of $p t p 2$ expression in $\Delta p t p 2$ SeMNPV-infected larvae, an RT-PCR amplification was performed 
on total RNA extracted from infected single whole larvae at two days post infection (dpi). Infections of 3rd instar S. exigua larvae were performed using the same procedure and treatment as described above. At two dpi, a single larva was homogenized in $250 \mu \mathrm{L}$ Trizol reagent (Invitrogen). Total RNA purification and subsequent cDNA synthesis were performed as described before (Han et al., 2015). RT-PCR was performed using primer pairs to amplify (i) a 486 bp sequence within the ORF of the $S$. exigua host translation initiation factor eIF5A (van Oers et al., 1999) to verify that larval RNA was successfully extracted and that cDNA was synthesized (primer 14 and 15; Table S1); (ii) a 492 bp sequence within the coding sequence of the SeMNPV immediate early (iel) gene to confirm successful virus infection (primer 16 and 17, Table 1); (iii) a $365 \mathrm{bp}$ sequence encompassing the recombined loxP site to check for the correct deletion (the forward primer stretched from $27 \mathrm{bp}$ upstream of the $p t p 2$ start codon to the first $9 \mathrm{bp}$ of the $p t p 2 \mathrm{ORF}$, the reverse primer annealed from 109 to $129 \mathrm{bp}$ downstream of the $p t p 2$ stop codon) (primer 12 and 13; Table S1). For each sample, a non-RT control sample was included, in which water was added instead of reverse transcriptase in the RT step. In addition, for each PCR a negative control with only water and a positive control with purified WT SeBac10 as template were processed.

\section{Comparison of Virus Infectivity and $\mathrm{Ob}$ Yield}

\section{Virus titrations}

To confirm whether deletion of the $p t p 2$ gene affect BVs production in larval hemocytes, BVs titers in WT SeMNPV- and $\Delta p t p 2$ SeMNPV-infected larvae were compared at two dpi. Infections were performed using the same procedures and treatments as described above. The experiment was performed three times as three independent replicates and per replicate 70 larvae were infected for each treatment. Two microliter of hemolymph was collected at 48 hpi from each larva as described above. Samples from 50 larvae were pooled in a total volume of $4 \mathrm{~mL}$ PBS containing $0.1 \%$ PTU and filtered through a $0.45 \mu \mathrm{m}$ non-pyrogenic filter to remove hemocytes and potential microbial contaminants. The filtered sample was used for virus titration. The $50 \%$ tissue culture infectious dose $\left(\mathrm{TCID}_{50}\right)$ was determined using an endpoint dilution assay (EPDA) on Se301 cells (Goertz et al., 2016) and scored at 7 dpi for the presence of SeMNPV by looking at the cytopathic effect. The results were analyzed for significant differences in virus titers by a $t$ test in GraphPad Prism 5 using a 95\% confidence interval. 


\section{$\underline{\text { Infectivity assays }}$}

To see whether removal of the $p t p 2$ gene of SeMNPV affected viral infectivity, we performed infectivity assays to determine the infectivity of WT and $\Delta p t p 2$ SeMNPV in 3rd instar S. exigua larvae as described before (Han et al., 2015). Five different concentrations were included for each virus, in 6-fold serial dilutions: $1.3 \times 10^{6}, 2.0 \times 10^{5}, 3.6 \times 10^{4}, 6.0 \times 10^{3}$, and $1.0 \times 10^{3}$ $\mathrm{OBs} / \mathrm{mL}$. Infections were performed as described above. Mock-infected larvae fed with a virusfree solution were used as controls. Larvae were scored for mortality from three dpi onwards until all larvae had died or pupated. Larvae that died for other reasons than virus infection were excluded from the analysis. The assays were performed three times. The program R v3.0.0 (R Core Team, 2013) was used for analyzing the data using a logistic regression model as described before (Ros et al., 2015). Treatment was used as a fixed effect and the model followed a binomial distribution.

To compare the time to death for WT and $\Delta p t p 2$ SeMNPV in 3rd instar S. exigua larvae, 48 larvae were infected with an concentration of $10^{6} \mathrm{OBs} / \mathrm{mL}$, known to kill at least $90 \%$ of infected larvae as described above. Mock-infected larvae were included as controls. Larvae were checked for mortality twice per day as described above and three independent replicates were performed. The effects of treatment and experiment on time to death were analyzed using Cox's proportional model in the program $\mathrm{R}$ as described before (Ros et al., 2015). Since most larvae died as 3 rd instars, larval stage was excluded as a factor in the model. LT $_{50}$ values were calculated in $\mathrm{R}$.

\section{Determination of OB yields}

Five larvae were randomly selected from each $\mathrm{LT}_{50}$ assay to determine the OB yield/larva. In total, 15 larvae were used for each virus (WT and $\Delta p t p 2$ SeMNPV). The cadavers were individually homogenized in $0.5 \mathrm{~mL}$ sterile water and then filtered through a double layer of cheese cloth. The filtrate was centrifuged at $6000 \mathrm{rpm}$ for $5 \mathrm{~min}$. The supernatant was discarded and the pellet was resuspended in $0.5 \mathrm{~mL}$ sterile water. OB yield/larva was calculated by counting the number of OBs in $10 \mu \mathrm{L}$ virus solutions using a Bürker-Türk hemocytometer. The counting was performed three times and the average was used for calculating the OB yield per larva. The OB yield was analyzed (virus treatment was used as a fixed factor) by a $t$ test in GraphPad Prism 5 using a 95\% confidence interval. 


\section{Results}

Transient expression of SeMNPV ptp2 in Sf21 cells induces mild apoptosis

Since the protein tyrosine phosphatase gene ( $p t p-H 2)$ from the polydnavirus MdBV showed pro-apoptotic effects on host cells, we hypothesized that SeMNPV ptp2 might function in a similar way. To investigate the pro-apoptotic effects of the SeMNPV ptp2 gene, we performed transient expression assays in Sf21 cells, using plasmids from which ptp2 was expressed together with EGFP (see Figure 1A). Based on the number of EGFP-expressing cells, transfection efficiencies were estimated to be $40-50 \%$ for each tested plasmid. The negative controls included mock-transfected cells, cells transfected with the modified 'empty' expression vector pIB-DESTmod (to check whether expression vector itself induces apoptosis in transfected cells), and cells transfected with pIB-EGFP expressing only EGFP, and in these treatments the cells did not show signs of apoptosis (Figure 1B; Figure S1). As a positive control, cells transfected with pIB-EGFP were treated with the strong apoptosis inducer ActD and morphological changes characteristic for apoptotic cells (cell blebbing and formation of apoptotic bodies) were observed (Figure 1B; Figure S1). Similarly, cell blebbing and apoptotic bodies were observed in cells transfected with pIB-SePTP2, but to a lesser extent compared with cells treated with ActD (Figure 1B; Figure S1). In contrast, cells transfected with pIBSePTP2mut, expressing a catalytic mutant of PTP2, did not show the formation of apoptosis and appeared healthy (Figure 1B; Figure S1). Signs of apoptosis were not observed in cells transfected with pIB-AcPTP, coding for the distantly related AcMNPV PTP gene (Figure S1). These results indicate that transient expression of SeMNPV PTP2 caused apoptosis in Sf21 cells. Since transfection with the catalytic mutant did not result in apoptotic cells, we conclude that the phosphatase activity of SePTP2 is needed to induce apoptosis. 
A

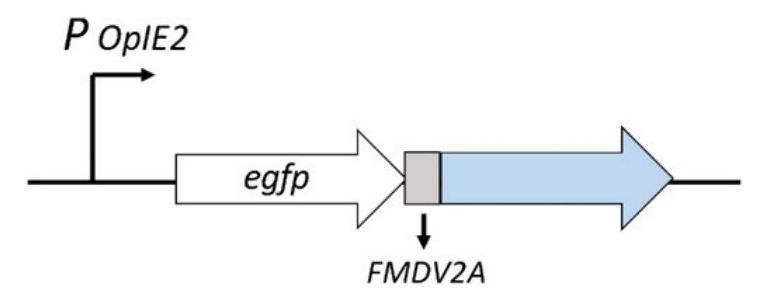

\section{B}
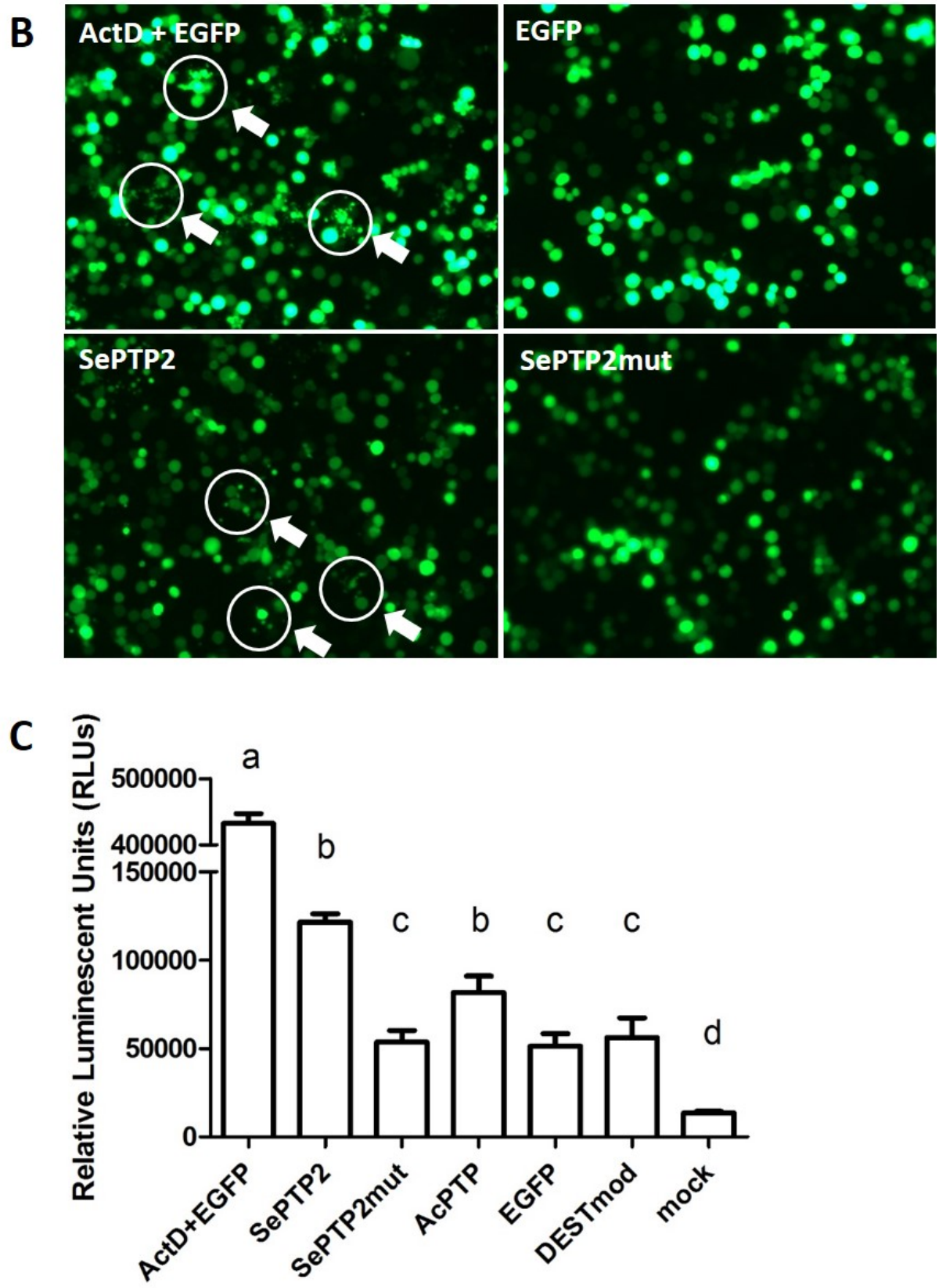

Figure 1. Transient expression of the Spodoptera exiguaprotein tyrosine phosphatase 2 (SePTP2) induced mild apoptosis in Sf21 cells. (A) Overview of the expression cassette in the pIB-DEST vector: $e g f p$ and the gene of interest were separated by the foot and mouth disease virus (FMDV) $2 \mathrm{~A}$ region and expression of the cassette was driven by the OpIE2 promoter; (B) EGFP expression in Sf21 cells at $48 \mathrm{~h}$ post-transfection (hpt) with pIB-EGFP + ActD (ActD + EGFP), pIB-EGFP (EGFP), pIB- 
SePTP2 (SePTP2), or pIB-SePTP2mut (SePTP2mut), respectively. Apoptotic bodies are indicated by white circles and arrows, scale bar $=200 \mu \mathrm{m}$; (C) caspase activity levels in Sf21 cells transfected with pIB-EGFP + ActD (ActD + EGFP), pIB-SePTP2 (SePTP2), pIB-SePTP2mut (SePTP2mut), pIB-AcPTP (AcPTP), pIB-EGFP (EGFP), pIB-DESTmod (DESTmod) and mock. Caspase 3/7 activity in transfected Sf2 1 cells was measured as relative luminescent units (RLUs) at $48 \mathrm{hpt}$, relative to a blank containing cell medium. Error bars represent the standard error of the mean. Treatment groups marked with a different letter ( $a, b, c$ or d) are significantly different (Table S2).

\section{SePTP2 induces apoptosis by caspase activation}

Caspases are a family of cysteine proteases that play an essential role during the induction of apoptosis. To study whether caspases are activated in the presence of PTP2, we measured the activity of the effector caspases 3 and 7 at $48 \mathrm{hpt}$. This activity was quantified by interaction of the effector caspases 3 and 7 with caspase-glo 3/7 substrates, which generates a luminescent signal (expressed in RLUs). The positive control expressing EGFP in the presence of ActD $($ ActD + EGFP) showed the highest caspase activity, corresponding to approximately 430,000

RLUs (Figure 1C). This level of activity was significantly higher compared to all other treatments $(P<0.001$ for all comparisons; Table S2). Cells expressing SePTP2 showed caspase activity levels of approximately 120,000 RLUs (Figure 1C), which was significantly lower than for the ActD + EGFP treatment $(P<0.001)$, but significantly higher than for the other treatments $(P<0.005$ for all comparisons; Table S2), except for AcPTP $(P=0.149)$. Mutation of the SePTP2 catalytic domain decreased the caspase activity level significantly to approximately 54,000 RLUs $(P<0.001)$. Cells transfected with pIB-EGFP and pIB-DESTmod, showed similar low levels of caspase activity as cells transfected with pIB-SePTP2mut (Figure $1 C ; P=1.000$ for both comparisons). For both replicates, cells transfected with a pIB-DEST vector (ActD + EGFP, SePTP2, SePTP2mut, AcPTP, EGFP, or DESTmod) showed higher levels of caspase activity than the mock transfection (Figure $1 \mathrm{C} ; P<0.001$ for all comparisons; Table S2). The results indicate that caspase activities were significantly higher in cells transfected with pIB-SePTP2 than cells transfected with other plasmids (except for cells transfected with pIB-AcPTP), supporting the conclusion that SePTP2, but also AcPTP, induces mild apoptosis upon transient expression in Sf21 cells. However, for AcPTP, no visual signs of apoptosis (apoptotic bodies or cell blebbing) were observed (Figure S1).

To confirm the involvement of caspases in SePTP2-induced apoptosis, we repeated the transfection experiment with pIB-SePTP2 in the presence or absence of the caspase inhibitor Z-VAD-FMK. In the absence of caspase inhibitor, cells transfected with pIB-SePTP2 again 
showed induction of apoptosis (Figure 2A). However, the presence of the caspase inhibitor blocked the induction of apoptosis: cells appeared to be healthy and cell blebbing and apoptotic bodies were not observed (Figure 2B). These data provide further evidence that SePTP2 induces apoptosis in Sf21 cells via caspase activation.
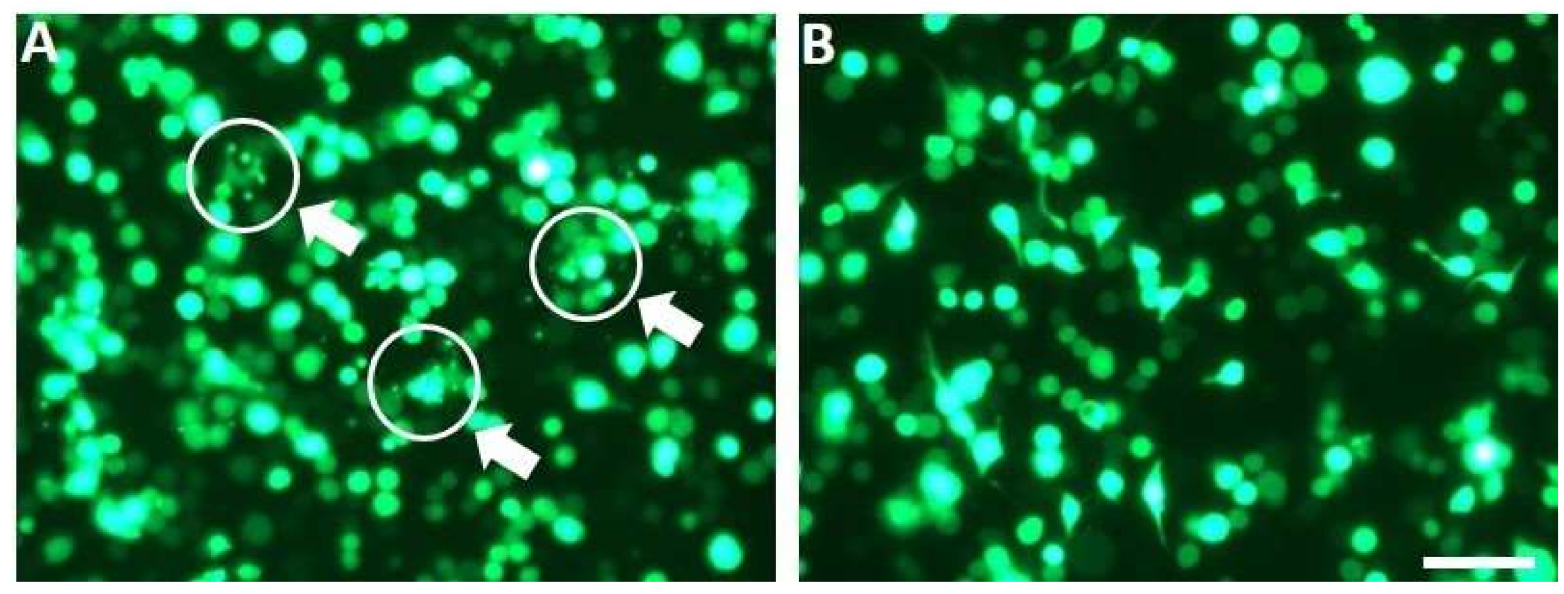

Figure 2. Caspase inhibitor Z-VAD-FMK blocked induction of apoptosis in $\mathrm{Sf} 21$ cells expressing SePTP2. EGFP expression in Sf21 cells at $48 \mathrm{~h}$ post transfection with pIB-SePTP2 (A) or with pIBSePTP2 + Z-VAD-FMK (B). Apoptotic bodies are indicated by white circles and arrows, scale bar $=$ $200 \mu \mathrm{m}$.

Deletion of the ptp2 gene reduces SeMNPV-induced apoptosis in $\mathrm{S}$. exigua hemocytes

To investigate whether SePTP2 also has a pro-apoptotic effect in hemocytes of S. exigua larvae, a mutant SeMNPV virus lacking the $p t p 2$ gene ( $\triangle p t p 2$ SeMNPV) was created. Hemocytes were collected from WT and $\Delta p t p 2$ SeMNPV-infected larvae at 48 hpi and then stained with Annexin V-EGFP (to stain apoptotic cells) and Hoechst (to stain the nuclei of all collected cells). Fluorescence microscopy analysis showed that WT SeMNPV also induced apoptosis in $S$. exigua hemocytes: The green Annexin V-EGFP signal was observed in many cells, indicating that these cells went into apoptosis (Figure 3). In contrast, hemocytes isolated from $\Delta p t p 2$ SeMNPV-infected and mock-infected larvae showed a much lower number of green cells, indicating apoptosis was induced at a much lower level by these treatments (Figure 3). 
Hoechst channel

GFP channel

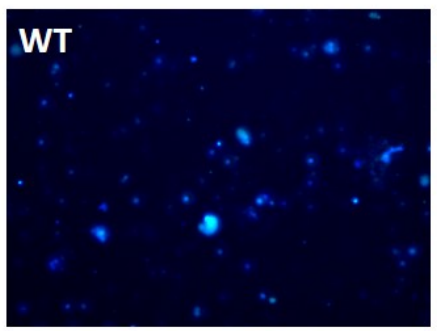

$\Delta p t p 2$

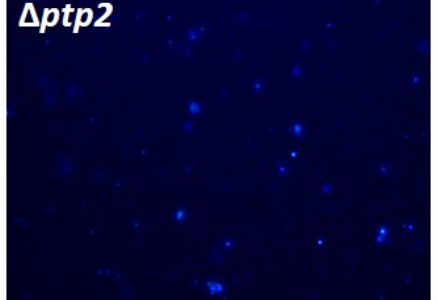

mock

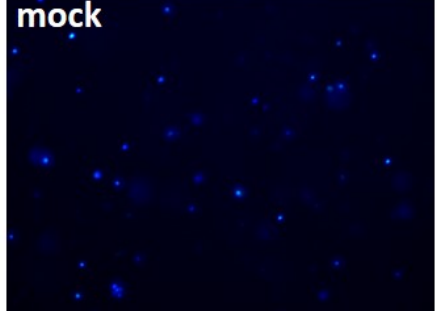

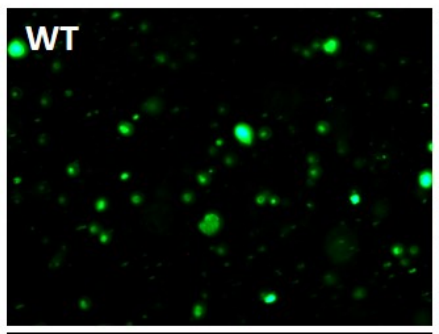

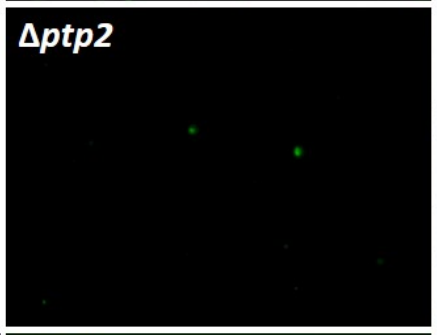

mock

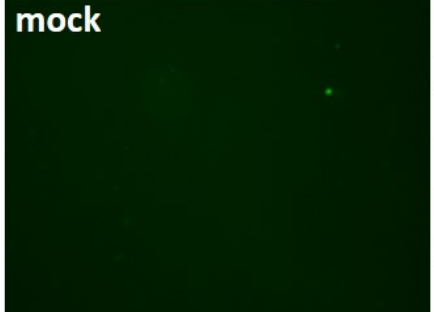

\section{Brightfield}

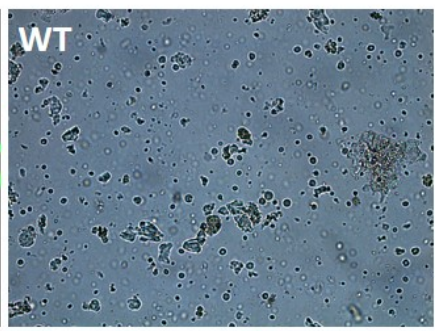

$\Delta \dot{p} t \dot{p} 2$

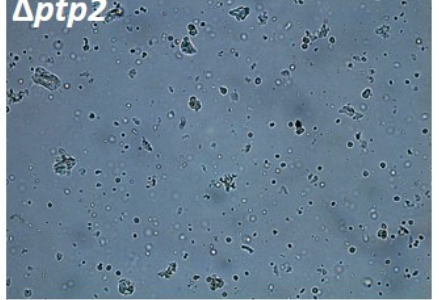

mock

Figure 3. Fluorescence microscopy analysis of cells in $S$. exigua hemocytes at $48 \mathrm{~h}$ post transfection. Cells were stained with Hoechst (left panel) and Annexin V-EGFP (middle panel). Cells were obtained from larvae infected with wild-type (WT) Spodoptera exigua multiple nucleopolyhedrovirus (SeMNPV), $\Delta p t p 2$ SeMNPV or no virus (mock), scale bar $=200 \mu \mathrm{m}$.

To confirm the expression of ptp2 in WT SeMNPV-infected larvae and to verify the absence of a full-length $p t p 2$ transcript in $\Delta p t p 2 \mathrm{SeMNPV}$-infected larvae, an RT-PCR was performed on total RNA extracted from mock- and virus-infected individual larvae at two dpi. As expected, a full-length $p t p 2$ transcript was present in WT SeMNPV-infected larvae and absent in mockinfected larvae (Figure 4, right panel, lanes 1 and 2). Partial deletion of 354 bp within the ptp2 ORF in $\triangle p t p 2$ SeMNPV resulted in a smaller product (Figure 4, right panel, lane 3) The SeMNPV iel gene, included as a control to check for successful virus infection (Figure 4, middle panel), was expressed in all virus-infected larvae and absent in mock-infected larvae. An RT-PCR targeting the mRNA of the S. exigua eIF5A gene was included as a control for correct RNA extraction and cDNA synthesis, and as expected eIF5A was expressed in both virus- and mock-infected larvae (Figure 4, left panel). 


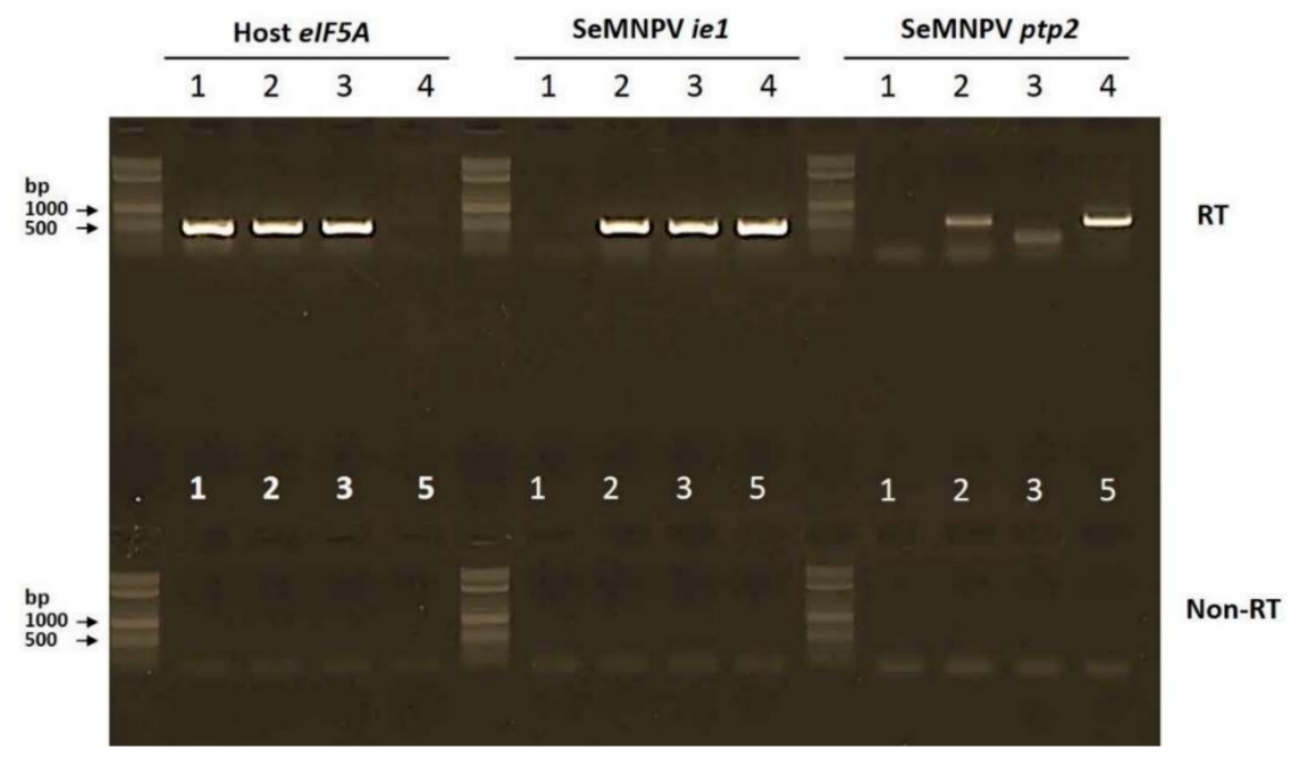

Figure 4. The full-length $p t p 2$ gene is expressed in WT SeMNPV-infected but not in $\triangle p t p 2$ SeMNPVinfected $S$. exigua larvae. RT-PCR analysis of mock-infected (1), WT SeMNPV-infected (2), or $\Delta p t p 2$ SeMNPV-infected (3), S. exigua larvae processed for RT-PCR analysis at two days post infection. For each PCR a WT SeMNPV bacmid control (4) and a water control (5) were included. Expression of the host eIF5A gene, the SeMNPV iel gene and the SeMNPV ptp2 gene were analyzed. For each RT sample, a PCR without RT step (non-RT) was performed in parallel. The 2-Log DNA Ladder (0.1-10.0 kbp, New England BioLabs Inc. Ipswich, MA, USA) was used in the agarose gel to estimate PCR fragment sizes.

\section{Virus infectivity was not affected by deleting the ptp2 gene}

To determine whether deletion of the ptp2 gene affected the production of infectious budded viruses (BVs), hemolymph of WT and $\Delta p t p 2$ SeMNPV-infected larvae was collected at $48 \mathrm{hpi}$ and the infectious BV titer was measured as the $\mathrm{TCID}_{50}$ value for $\mathrm{Se} 301$ cells for three biological replicates. The BV titers were not significantly different between the two treatments at 48 hpi $(t$ test $=1.314$; d.f. $=4 ; P=0.2592)$, indicating that deletion of the $p t p 2$ gene did not affect the production of infectious BVs (Table S3).

To study whether deletion of the viral ptp2 gene affected viral infectivity, we performed a logistic regression on the mortality data obtained after infecting S. exigua third instar larvae with WT and $\Delta p t p 2$ SeMNPV, for each replicate separately. The ratio of infectivity of $\Delta p t p 2$ SeMNPV to WT SeMNPV (odds ratio) was determined. In all three replicates, the infectivity of WT and $\Delta p t p 2$ SeMNPV were not significantly different (judged by overlap of $95 \%$ 
confidence interval of the odds ratio; Table S4). There were no significant differences in mortality between the three replicates.

We then investigated whether deletion of the viral ptp2 gene affected the time to death for infected third instars of $S$. exigua, using a survival analysis. The Cox's proportional hazards model was used to determine the mortality rate (hazard rate, rate at which larvae died) for WT and $\triangle p t p 2$ SeMNPV. Treatment and experiment were included as factors. The mortality rate for larvae infected with mutant virus was similar to that of WT SeMNPV-infected larvae (ratio of $1.05 ; \mathrm{z}=0.375 ; P=0.721)$. There were no significant differences between the three replicates.

\section{Deletion of the ptp2 gene decreases SeMNPV OB yields}

To determine the effect of deletion of the ptp2 gene on the total OB yield, we randomly selected 15 cadavers from WT and $\Delta p t p 2$ SeMNPV-deceased larvae. The OB yield of individual larvae was counted using a Bürker-Türk hemocytometer. The OB yield from larvae killed by WT SeMNPV was significantly higher that from larvae killed by $\triangle p t p 2 \mathrm{SeMNPV}$, in which the OB yield was decreased by $34 \%$ (Figure $5 ; t$ test $=2.583$; d.f. $=28 ; P<0.05$ ). Though deletion of the $p t p 2$ gene did not affect virus infectivity nor the time to death, the OB yield was lower for larvae infected with the mutant SeMNPV lacking $p t p 2$.

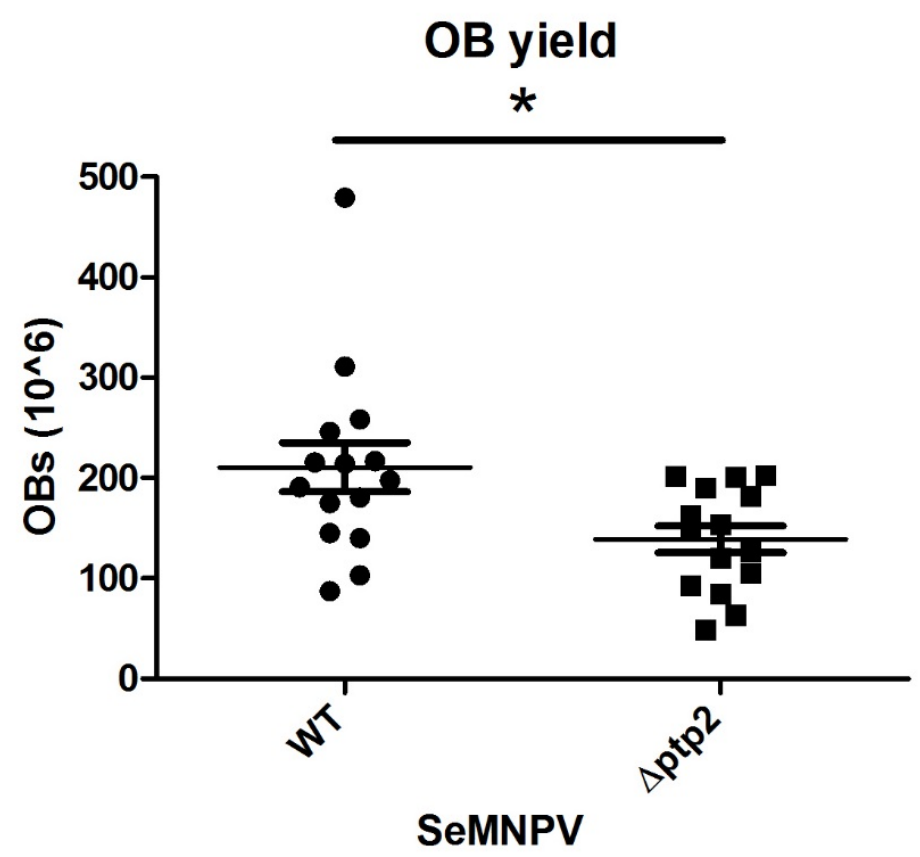

Figure 5. Occlusion body (OB) yield is significantly reduced in S. exigua larvae infected with $\Delta p t p 2$ SeMNPV compared to larvae infected with WT SeMNPV. Data points represent the OB yield of 
individual larvae. Horizontal lines show the mean value of OB yield and whiskers the standard error of the mean. Asterisk indicates significant difference ( $t$ test, $P<0.05$ ).

\section{Discussion}

Many viruses carry genes that are involved in inducing apoptosis in host-derived cell lines (Chitnis et al., 2011; Clem, 2001; Han et al., 2009; Prikhod'ko and Miller, 1996; Suderman et al., 2008), such as the ptp-h2 gene from MdBV, which induces apoptosis in Sf21 cells (Suderman et al., 2008). The baculovirus SeMNPV carries a protein tyrosine phosphatase gene (ptp2) which contains the same catalytic domain as $p t p-h 2$, therefore, we tested whether SeMNPV $p t p 2$ has a pro-apoptotic function as well. To test this hypothesis we took advantage of Sf21 cells, which are known to undergo apoptosis upon different stimuli (Clem et al., 1991). Sf21 cells transfected with a plasmid expressing SePTP2 showed morphological signs of apoptosis and increased levels of caspase activity. However, Se301 cells, Trichoplusia ni High Five cells and Drosophila S2 cells transfected with the pIB-SePTP2 plasmid did not undergo apoptosis (data not shown), indicating that the pro-apoptotic effect of SePTP2 might be restricted to certain cell types. Similar results were found for MdBV PTP-H2 as well; PTP-H2 induced apoptosis in Sf21 cells, but not in granulocytes or plasmatocytes of $S$. frugiperda larvae, T. $n i$ High Five cells or Drosophila S2 cells (Suderman et al., 2008). More interestingly, some viruses even induce apoptosis in one cell type, but block apoptosis formation in another cell type. For example, Herpes simplex virus (HSV) induces apoptosis in Jurkat cells, a T-cell leukemia line, but protected HEp-2 cells (a carcinoma cell line) from apoptosis triggered by tumor necrosis factor alpha (Han et al., 2009). To our knowledge, there is no explanation so far why a viral gene induces apoptosis in one cell type, but not in another cell type. A possible explanation is that certain regulatory pathways are not active in cell types in which apoptosis is not induced. As a result, the pro-apoptotic effect of viral genes is restricted to certain cell types or host tissues.

Our data showed a significantly increased caspase activity in transfected cells expressing SePTP2. In addition, the phosphatase activity of SePTP2 was needed to activate the effector caspases. Cells expressing the distantly related baculovirus PTP (AcPTP) also showed increased caspase activity; however, apoptosis was not observed in these cells (absence of cell blebbing and apoptotic bodies), indicating that the pro-apoptotic effect was not an overall effect of transient expression of baculovirus PTPs. Similar results were also observed for MdBV; MdBV encodes 13 different PTPs, but only PTP-H2 showed a pro-apoptotic effect in Sf21 cells 
(Pruijssers and Strand, 2007; Suderman et al., 2008). SePTP2 protein possesses a C-terminal consensus sequence that is characteristic for MAPK phosphatases, which remove the phosphoresidue from MAPKs. There are three major MAPK pathways: extracellular signal-regulated kinase (ERK), c-Jun N-terminal kinase (JNK) and p38. All three pathways have been reported to be involved in regulating apoptosis (Abdelwahid et al., 2007; Kim et al., 2008; Porras et al., 2004). Therefore, SePTP2 may induce apoptosis by regulating MAPK activity in cells. Further studies are needed to identify the substrates of SePTP2 to confirm this.

In our study, deletion of the ptp2 gene severely reduced SeMNPV-induced apoptosis in $S$. exigua hemocytes, while the SeMNPV BV titer, virus infectivity and time to death were not affected upon deletion of $p t p 2$. However, the OB yield was significantly higher from larvae infected with WT SeMNPV than from larvae infected with $\triangle p t p 2$ SeMNPV. A previous study on BmNPV showed that ERK- and JNK-dependent signaling pathways contribute to BmNPV virus yield; knocking down the Bombyx mori erk or jnk genes reduced the production of both OBs and BVs (Katsuma et al., 2007). However, whether (and which) viral genes are involved in activating the host ERK- and JNK-pathways during a BmNPV infection is not known. SePTP2 may function as a MAPK phosphatase that regulates host MAPK pathways that eventually affect $\mathrm{OB}$ yield in infected hosts. Virus-induced apoptosis is normally regarded as a defense mechanism against virus infection; however, some viruses stimulate apoptosis in immune cells to maximize viral fitness and transmission (Clarke and Tyler, 2009; Jaworska et al., 2014). For example, the influenza A virus (IAV) induces apoptosis in innate immune cells at the early stage of infection to subvert host immunity (Jaworska et al., 2014). Similar results were also found in MdBV infected S. frugiperda larvae (Suderman et al., 2008). We hypothesize that the induction of apoptosis by SeMNPV PTP2 suppresses the $S$. exigua larval immune system and by doing so the infected larvae accumulate more OBs. These higher OB yields most likely benefit virus dissemination, and hence transmission.

It seems contradictory that viruses carry both anti-apoptotic and pro-apoptotic genes. Baculoviruses are known to produce anti-apoptotic proteins (e.g., P35 and IAP) to suppress or delay apoptosis (Clem, 2001). For example, besides SePTP2, SeMNPV also encodes the antiapoptotic proteins IAP2 and IAP3 (Wu et al., 2016; Zhu et al., 2008). However, anti-apoptotic and pro-apoptotic genes may function via different pathways. While anti-apoptotic genes counteract host-induced apoptosis (which interferes with viral replication in general), allowing the virus to replicate and eventually increasing virus progeny, pro-apoptotic genes might serve 
to suppress the host immune system by targeting specific cell types. Future studies should show whether the induction of apoptosis in S. exigua larvae is restricted to hemocytes.

Overall, we conclude that the SeMNPV ptp2 gene functions as a pro-apoptotic gene in cultured Sf21 insect cells, inducing mild apoptosis, and that the phosphatase activity of SePTP2 is needed for this process. Furthermore, we showed that the SeMNPV ptp2 gene is involved in inducing apoptosis in host hemocytes. Viral suppression of host immunity eventually contributes to a higher OB yield.

\section{Acknowledgements}

We thank Susan van Aalst for her assistance with constructing the pIB-SePTP2 plasmid and with initial apoptosis assays. Gorben Pijlman is acknowledged for useful discussions throughout the experiments. Els Roode's help in cell culture is highly appreciated. 


\section{Supplementary Materials}

Table S1. Primers used in this study.

\begin{tabular}{|c|c|c|c|}
\hline $\begin{array}{l}\text { Primer } \\
\text { number }\end{array}$ & Primer name & Sequence & Aim \\
\hline 1 & $\begin{array}{l}\text { pIB- } \\
\text { DESTmod- } \\
\text { fw }\end{array}$ & 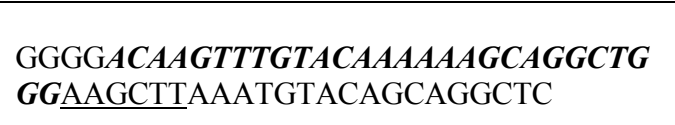 & $\begin{array}{l}\text { Amplify Acgp64 segment with attB1 and } \\
\text { HindIII site }\end{array}$ \\
\hline 2 & $\begin{array}{l}\text { pIB- } \\
\text { DESTmod- } \\
\text { rv }\end{array}$ & 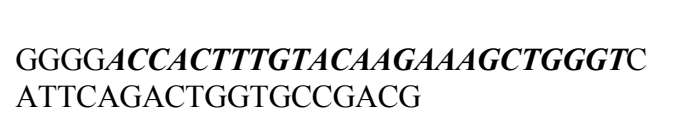 & Amplify Acgp64 segment with attB2 site \\
\hline 3 & $\begin{array}{l}\text { pIB-Acptp- } \\
\text { fw }\end{array}$ & 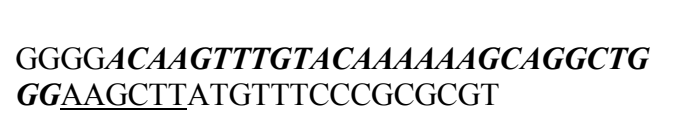 & $\begin{array}{l}\text { Amplify Acptp ORF with attB1 and } \\
\text { HindIII site }\end{array}$ \\
\hline 4 & $\begin{array}{l}\text { pIB-Acptp- } \\
\text { rv }\end{array}$ & $\begin{array}{l}\text { GGGGACCACTTTGTACAAGAAAGCTGGG } \\
\text { TTTAAATTAATAAATCTTGAACGTAATTTTG } \\
\text { TC }\end{array}$ & Amplify Acptp ORF with attB2 site \\
\hline 5 & $\begin{array}{l}\text { pIB-Septp2- } \\
\text { fw }\end{array}$ & 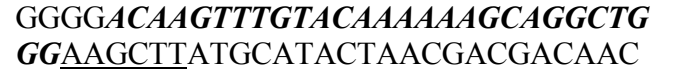 & $\begin{array}{l}\text { Amplify Septp2 and Septp } 2^{C 110 S} \text { ORF with } \\
\text { attB1 and HindIII site }\end{array}$ \\
\hline 6 & $\begin{array}{l}\text { pIB-Septp2- } \\
\text { rv }\end{array}$ & 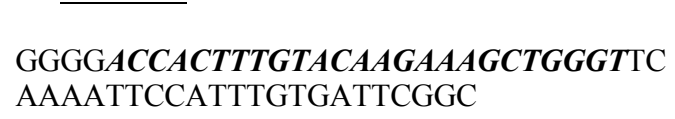 & $\begin{array}{l}\text { Amplify Septp2 and Septp } 2^{\text {ClloS }} \text { ORF with } \\
\text { attB2 site }\end{array}$ \\
\hline 7 & $\begin{array}{l}p t p 2^{\text {Cl10S }} \\
\text { fw1 }\end{array}$ & $\begin{array}{l}\text { GGCGAGGGCAAAAGGGTGTACGTTCATAG } \\
\text { CCATGCGGGCGTGTCG }\end{array}$ & $\begin{array}{l}\text { Create } \mathrm{C} 110 \mathrm{~S} \text { mutation in SePTP2 } \\
\text { catalytic site }\end{array}$ \\
\hline 8 & $p t p 2^{C l 10 S_{-r v}}$ & TCAAAATTCCATTTGTGATTC & Amplify Septp2 ORF \\
\hline 9 & $\begin{array}{l}p t p 2^{\text {CllOS }_{-}} \\
\text {fw2 }\end{array}$ & ATGCATACTAACGACGACAAC & Amplify Septp2 ORF \\
\hline 10 & $\Delta p t p 2-\mathrm{fw}$ & $\begin{array}{l}\text { ATTATAGTTTACGTCGCCGACGAAACAATC } \\
\text { ATTGATATCGTGGACACGAGCTCGGATCCA } \\
\text { CTAGTAACGG }\end{array}$ & Create $p t p 2$ ORF deletion \\
\hline 11 & $\Delta p t p 2-\mathrm{rv}$ & $\begin{array}{l}\text { ACTATGCATACTAACGACGACAACTTTACG } \\
\text { CAACTGTCCAACGGAAACTGCCTCTAGATG } \\
\text { CATGCTCGAG }\end{array}$ & Create $p t p 2$ ORF deletion \\
\hline 12 & $\begin{array}{l}\text { Aptp2- } \\
\text { check-fw }\end{array}$ & ATGTGTGCTCTTCGTCAGATG & $\begin{array}{l}\text { Check } p t p 2 \text { ORF deletion and RT-PCR on } \\
\text { SeMNPV } p t p 2\end{array}$ \\
\hline 13 & $\begin{array}{l}\triangle p t p 2- \\
\text { check-rv }\end{array}$ & $\begin{array}{l}\text { TTCAATATAATAAGAAGAACACTATGCATA } \\
\text { CT }\end{array}$ & $\begin{array}{l}\text { Check ptp } 2 \text { ORF deletion and RT-PCR on } \\
\text { SeMNPV ptp2 }\end{array}$ \\
\hline 14 & $\mathrm{Se}-\mathrm{eIF} 5 A$-fw & GCCATGGCTGACATCGAGGATAC & RT-PCR on $S$. exigua eIF $5 \mathrm{~A}$ \\
\hline 15 & Se-eIF5A-rv & GCGGTACCGGTTTATTTGTCGAGAGC & RT-PCR on S. exigua eIF5A \\
\hline 16 & Se-iel-fw & GACAAGAATGACGATGATATCGG & RT-PCR on SeMNPV iel \\
\hline 17 & Se-iel-rv & GGACAATTGCTTTTCCGAAAAC & RT-PCR on SeMNPV iel \\
\hline
\end{tabular}


Table S2: TCID50 value in the hemolymph of WT- and $\Delta p t p 2$ - infected larvae at $48 \mathrm{hpi}$

\begin{tabular}{llll}
\hline & $\begin{array}{l}\text { Replicate } 1 \\
\text { (TCID50/ml) }\end{array}$ & $\begin{array}{l}\text { Replicate 2 } \\
\text { (TCID50/ml) }\end{array}$ & $\begin{array}{l}\text { Replicate 3 } \\
\text { (TCID50/ml) }\end{array}$ \\
\hline WT SeMNPV & $8.91 * 10^{6}$ & $1.20^{*} 10^{7}$ & $2.11^{*} 10^{7}$ \\
$\Delta p t p 2$ SeMNPV & $1.99 * 10^{7}$ & $1.58 * 10^{7}$ & $4.47^{7} 10^{7}$ \\
\hline
\end{tabular}

Table S3: Outcome of the logistic regression analysis of the infectivity assays. Per replicate, an odds ratio (relative potency) was determined: the ratio of the infectivity of $\Delta p t p 2$ SeMNPV relative to the infectivity of the WT virus. The upper and lower limits of the $95 \%$ confidence interval are also given, as well as the $\mathrm{P}$ value.

\begin{tabular}{llllll}
\hline Replicate & Viruses & Odds ration & \multicolumn{2}{l}{ 95\% Confidence Interval } & P value \\
\hline 1 & & Low & High & \\
\hline \multirow{2}{*}{2} & WT SeMNPV & 1.000 & - & - & - \\
& $\Delta p t p 2$ SeMNPV & 1.616 & 0.946 & 1.419 & 0.081 \\
3 & WT SeMNPV & 1.000 & - & - & - \\
& Dptp2 SeMNPV & 1.393 & 0.876 & 2.225 & 0.163 \\
& WT SeMNPV & 1.000 & - & - & - \\
& $\Delta p t p 2$ SeMNPV & 1.120 & 0.687 & 1.831 & 0.649 \\
\hline
\end{tabular}

Table S4: Outcome of the logistic regression analysis of the infectivity assays. Per replicate, an odds ratio (relative potency) was determined: the ratio of the infectivity of $\Delta \mathrm{ptp} 2 \mathrm{SeMNPV}$ relative to the infectivity of WT SeMNPV. The upper and lower limits of the $95 \%$ confidence interval are also given, as well as the $\mathrm{P}$ value.

\begin{tabular}{llllll}
\hline Replicate & Viruses & Odds ratio & \multicolumn{2}{l}{ 95\% Confidence Interval } & P value \\
\hline 1 & & Low & High & \\
\hline \multirow{2}{*}{2} & WT SeMNPV & 1.000 & - & - & - \\
& Aptp2 SeMNPV & 1.616 & 0.946 & 1.419 & 0.081 \\
3 & WT SeMNPV & 1.000 & - & - & - \\
& Aptp2 SeMNPV & 1.393 & 0.876 & 2.225 & 0.163 \\
& WT SeMNPV & 1.000 & - & - & - \\
& $\Delta p t p 2$ SeMNPV & 1.120 & 0.687 & 1.831 & 0.649 \\
\hline
\end{tabular}




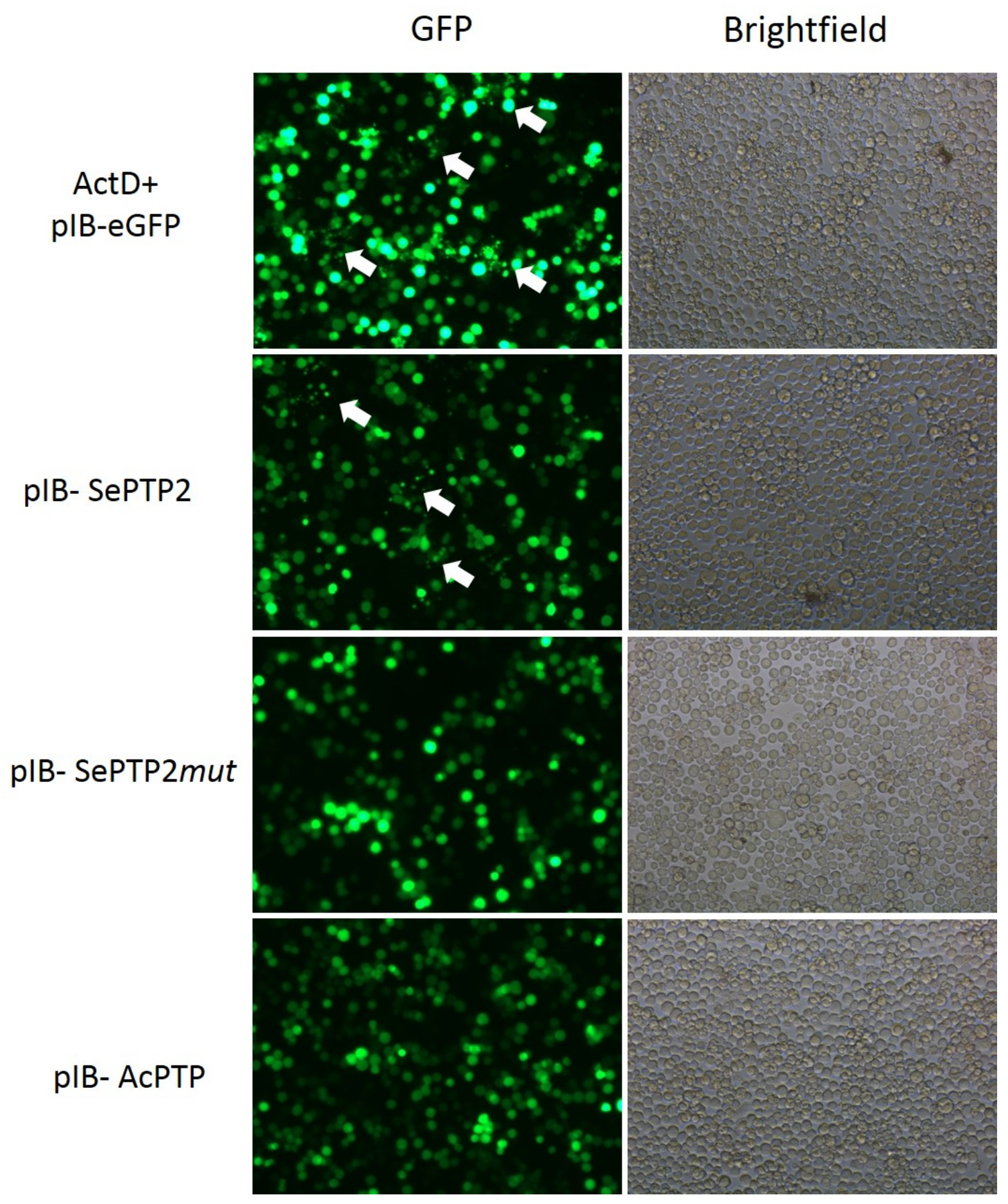




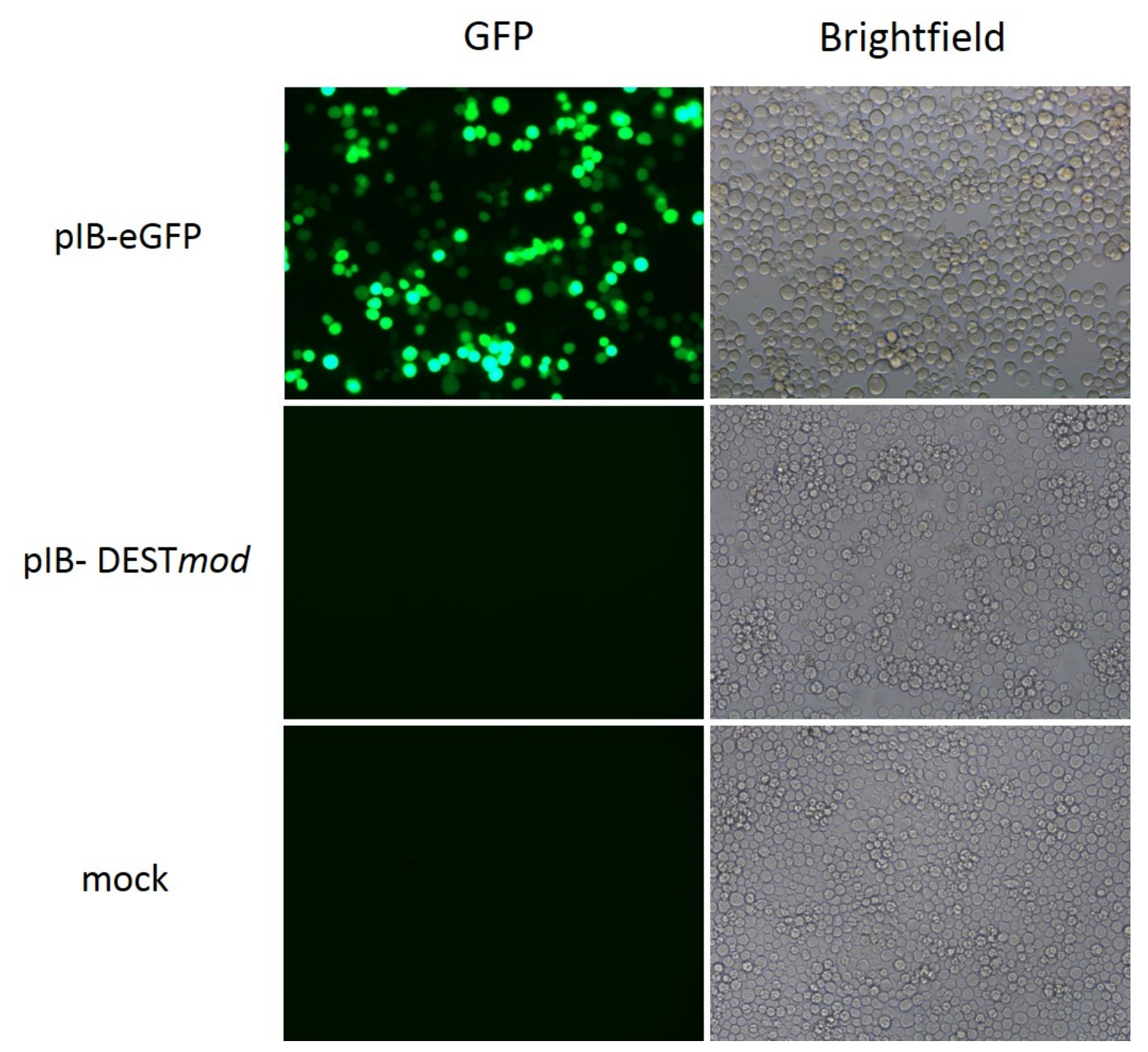

Figure 1. Microscopy analysis of transfected Sf21 cells at 48 hours post transfection (hpt). Left panel was the GFP channel, right panel was the brightfield channel. Treatments were ActD + pIBeGFP, pIBSePTP2, pIB-SePTP2mut, pIB-AcPTP, pIB-eGFP, pIB-DESTmod, mock. Apoptosis bodies were indicated by white arrows. 


\section{Chapter}

Substrate identification of baculovirus protein tyrosine phosphatase

Han Y, Boeren S, Ros VID and van Oers MM. Manuscript in preparation. 


\begin{abstract}
Baculoviruses contain both protein kinase and phosphatase genes in their genome and protein phosphorylation and dephosphorylation plays a significant role during baculovirus replication. Autographa californica multiple nucleopolyhedrovirus (AcMNPV) encodes a $19 \mathrm{kDa}$ protein tyrosine phosphatase (PTP) enzyme, which in vitro removes phosphate groups from tyrosine, serine and threonine residues and which shows a high affinity for RNA substrates. Deletion of the $p t p$ gene from the viral genome did not affect virus replication in general, nor virus virulence or time to death of infected hosts. On the other hand, previous work has demonstrated that AcMNPV PTP induces hyperactivity in infected Spodoptera exigua larvae. To investigate which viral and/or host proteins might be targeted by PTP, we performed a coimmunoprecipitation assay using anti-GFP antibodies to pull down GFP-tagged PTP proteins from infected larvae. In total, thirteen viral proteins and five host proteins that co-purified with PTP were identified with liquid chromatography-mass spectrometry proteomic analysis. Several of these proteins are known to be important for nucleocapsid assembly, nucleocapsid transport, ODV envelopment and/or virus dissemination and some of these may potentially be involved in PTP-mediated hyperactivity of infected larvae. The results suggest that AcPTP functions both as a structural protein and as a phosphatase enzyme. We hypothesize that AcPTP may control virus entry into certain host tissues, for example the host brain, ultimately leading to the behavioural change in the infected insects.
\end{abstract}




\section{Introduction}

Baculoviruses are a diverse group of viruses that mainly infect insects in the orders Lepidoptera, Diptera and Hymenoptera (Jehle et al., 2006; Rohrmann, 2013; Slack and Arif, 2007). Baculoviruses contain large, double-stranded, circular, supercoiled genomes. The genomes are 80 to $180 \mathrm{~kb}$ in size and may contain 90 to 180 genes (Slack and Arif, 2007). During an infection cycle, two types of virions are formed: budded viruses (BVs) and occlusion-derived viruses (ODVs). The BVs are responsible for cell to cell infection and ODVs are responsible for infection from individual to individual, as reviewed by Rohrmann (2013). Baculovirus genes are categorized into immediate-early, early, late and very late genes. Expression of immediateearly and early genes prepares for the viral DNA replication initiation and genes for structural proteins are mainly expressed in the late stage of infection (Cohen et al., 2009; Slack and Arif, 2007).

Protein phosphorylation and dephosphorylation plays a significant role in baculovirus replication (Li and Guarino, 2008; Reilly and Guarino, 1994). The most well-studied baculovirus, Autographa californica multiple nucleopolyhedrovirus (AcMNPV), encodes two protein kinases, PK1 and PK2, with homology to eukaryotic protein kinases (Li and Miller, 1995). AcMNPV also contains a protein tyrosine phosphatase ( $p t p)$ gene in its genome. The $p t p$ gene encodes the $19 \mathrm{kDa}$ protein tyrosine phosphatase (PTP) enzyme that contains a $\mathrm{HC}$ signature motif (Li and Guarino, 2008). The AcPTP enzyme dephosphorylates protein substrates at tyrosine, serine and threonine residues in vitro (Sheng and Charbonneau, 1993). Moreover, AcMNPV PTP was shown to function as an 5'-RNA phosphatase and has a higher affinity for RNA than for protein substrates (Takagi et al., 1998). AcPTP sequentially removes $\gamma$ and $\beta$ phosphates from the 5'-end of triphosphate-terminated RNA, leaving a 5'monophosphate end ( $\mathrm{Li}$ and Guarino, 2008; Takagi et al., 1998). Ptp genes are present in a subset of the alphabaculoviruses, named group I nucleopolyhedroviruses (NPVs).

Another important function of PTP relates to host behavioural manipulation. Two types of behavioural changes are induced in lepidopteran hosts upon baculovirus infection: hyperactivity (enhanced locomotion behaviour) and pre-death climbing behaviour (tree-top disease), as reviewed by Han et al. (2015b) and van Houte et al. (2013). These behavioural changes are thought to optimize virus dissemination. Previous studies have demonstrated the involvement of the baculovirus ptp gene in inducing hyperactivity in infected caterpillars (Kamita et al. 2005, van Houte et al. 2012). The ptp gene from Bombyx mori 
nucleopolyhedrovirus (BmNPV) is responsible for inducing hyperactivity in infected B. mori larvae (Kamita et al., 2005). These authors demonstrated that the phosphatase activity of the encoded BmPTP protein was not needed for this process (Kamita et al., 2005; Katsuma et al., 2012). The ptp gene from the baculovirus AcMNPV triggers hyperactivity in Spodoptera exigua larvae (van Houte et al., 2012). However, in this system, the phosphatase activity of the AcPTP protein is required for inducing the altered behaviour, as demonstrated using a PTP catalytic-site mutant. Hosts infected with this catalytic mutant did not become hyperactive (van Houte et al., 2012). The fact that the phosphatase activity of AcPTP is required for inducing hyperactivity in S. exigua larvae suggests that host or viral proteins serve as substrate(s) for the AcMNPV PTP enzyme and that dephosphorylation of the substrate(s) eventually leads to altered host behaviour.

In the current study, we aim to identify host and viral proteins that interact with AcMNPV PTP. To identify potential substrates, a so-called "substrate trapping" approach was used (Jia et al., 1995). In this approach, a mutant PTP enzyme is used in which the cysteine residue in the PTPsignature motif (HC) is mutated into an alanine. The mutation ensures proper binding of substrates, while the subsequent catalysis does not happen, thus trapping the substrate in the binding pocket of the mutant PTP protein (Jia et al., 1995). Green fluorescent protein (GFP) was added to the PTP protein as a tag for protein purification. Co-immunoprecipitation (Co-IP) using anti-GFP antibodies was performed on infected larvae, and subsequently the co-purified proteins were identified by tandem mass spectrometry (MS/MS). Both viral and host proteins were identified by this approach. We discuss the function of these proteins and speculate on their potential role in the baculovirus infection cycle and baculovirus-induced hyperactivity.

\section{Material and Methods}

\section{Insects and insect cell culture}

Spodoptera exigua larvae were reared on artificial diet as described before (Han et al., 2015a). Spodoptera frugiperda 9 (Sf9) cells (Sigma-Aldrich) were maintained as monolayers in Sf900II serum-free medium (Invitrogen) supplied with 5\% fetal bovine serum (FBS) (Invitrogen) and $0.1 \%$ gentamycin $(50 \mu \mathrm{g} / \mathrm{mL}$, Invitrogen $)$. 


\section{Generation of recombinant bacmids}

An AcMNPV bacmid with a deletion of the $p t p$ gene ( $\Delta p t p)$, derived from the wildtype (WT) bacmid, was previously provided by Linda Guarino of Texas A\&M University, USA (Li and Guarino, 2008), and was used as a backbone to produce the various PTP constructs. To enable purification of the (mutated) AcMNPV PTP protein from infected larvae, recombinant bacmids were created that would produce the PTP protein as a fusion to GFP (Figure 1A). To this aim, four recombinant bacmids were created: two bacmids producing PTP proteins with either a $\mathrm{C}$ or an N-terminal GFP-tag, and the other two bacmids producing a PTP catalytic mutant (PTPM) protein, again with either a $\mathrm{C}$ - or an N-terminal GFP-tag. In the PTPM mutant the cysteine (C) in the PTP HC signature motif was exchanged for an alanine (A) triplet ( $\left.p t p^{C 119 A}\right)$. An additional recombinant bacmid to make non-fused GFP was also constructed and served as a negative control (Figure 1A).

Two-step PCR amplifications were performed to make constructs encoding PTP or the PTPM protein with an N-terminal GFP tag. In the first PCR step, the $g f p$ ORF was amplified from the plasmid CHIKrep-pac2AGFP (Fros et al., 2010) using primers 1 and 2 (Supplementary Table S1). Simultaneously, the $p t p$ and ptpm ORFs were amplified, respectively, from the WT AcMNPV E2 bacmid (Smith and Summers, 1979) and the plasmid pFBDpolh $P_{\mathrm{gp} 64} p t p^{C 119 A}$ (van Houte et al., 2012), using primers 3 and 4 (Supplementary Table S1). Primers 2 and 4 both had $40 \mathrm{nt}$ overhangs, so that the last $40 \mathrm{bp}$ of the resulting $g f p$ product was complementary to the first $40 \mathrm{bp}$ of the resulting $p t p$ and $p t p m$ products. In the second round of PCR amplification, the products from the first PCR step were used as templates (1:1 molar ratio) and combined with primers 1 and 4 (Supplementary Table S1) to generate the $g f p$-ptp and gfp-ptpm fragments.

To make constructs encoding PTP or PTPM protein with a C-terminal GFP tag a similar approach was used. Two-step fusion PCRs were conducted to create the $p t p-g f p$ or $p t p m-g f p$ fusion products. In the first PCR step primers 5 and 6 (with 42 nt overhang; Supplementary Table S1) were used to amplify the $p t p$ and ptpm ORFs. Primers 7 (with 42 nt overhang) and 8 (Supplementary Table S1) were used to amplify the $g f p$ ORF. The last 42 bp of the resulting $p t p$ or $p t p m$ products was complementary to the first $42 \mathrm{bp}$ of the resulting $g f p$ products. In the second PCR, the $p t p-g f p$ or $p t p m-g f p$ fragments were generated by using the first step PCR products as templates (1:1 molar ratio) combined with primers 5 and 8 (Supplementary Table $\mathrm{S} 1)$. The $g f p$ fragment for the negative control bacmid was amplified from the plasmid CHIKrep-pac2AGFP (Fros et al., 2010) using primers 1 and 8. 
Subsequently, all five fragments ( $g f p-p t p$, $g f p-p t p m$, $p t p-g f p$, ptpm-gfp and $g f p$ ) were cloned into the pFBDpolh $P_{\text {gp64 }}$ plasmid between the NheI and NsiI restriction sites. By using this plasmid, the expression of the fusion-constructs will be controlled by the AcMNPV gp64 promoter $\left(P_{\mathrm{gp} 64}\right)$, which is active early and late during the infection (Whitford et al., 1989). The pFBDpolh $P_{\text {gp64 }}$ vector was derived from pFBDpolh $\Delta$ p10 (Peng et al., 2010) in which the AcMNPV polyhedrin (polh) ORF had been inserted downstream of the polh promoter and the p10 promoter was removed. The AcMNPV $P_{\text {gp64 }}$ promoter was cloned between the NcoI and NheI restriction sites remaining from the original p10 MCS to make the pFBDpolh $P_{\text {gp } 64}$ vector. To create the five recombinant bacmids, bac-to-bac transposition (Luckow et al., 1993) was performed using the AcMNPV $\Delta p t p$ backbone described above.

\section{Proliferation and purification of virus}

To obtain recombinant viruses, Sf9 cells were transfected with the recombinant bacmids using Expres2TR reagent (Expres2ion Biotechnologies). The BVs produced were amplified once in Sf9 cells. Viral occlusion bodies (OBs) were produced in Sf9 cells by infecting cells with an MOI of 5 TCID $_{50}$ units per cell. An OB stock was generated by feeding OB pellets collected from cell debris to $S$. exigua fourth instars using the droplet feeding method (van Houte et al. 2012). OB purification was performed as described before (Han et al., 2015a).

\section{Sample preparation and LC-MS/MS measurements}

Newly moulted S. exigua third instars were infected with a concentration of $10^{8} \mathrm{OBs} / \mathrm{ml}$ using droplet feeding (van Houte et al., 2012). All assays consisted of three biological replicates. Forty-eight hours post infection (hpi), five infected larvae were collected, pooled and homogenized using a plastic pestle in $500 \mu 1$ lysis buffer (10 mM Tris pH 7.5, 1\% NP40, 150 $\mathrm{mM} \mathrm{Nacl,} \mathrm{20 \%} \mathrm{glycerol,} 1 \mathrm{mM}$ DTT, proteinase inhibitor). GFP-tagged proteins were purified using the $\mu \mathrm{MACS}^{\mathrm{TM}}$ Epitope Tag Protein Isolation Kit (Miltenyi Biotech) as described in Wendrich et al. (2017). Proteins were eluted in pre-heated elution buffer from the kit. The precipitation experiments were conducted three times from the same starting materials. Equal volumes of eluted samples were loaded onto 12\% SDS-PAGE gels and stained with Coomassie Brilliant Blue. All samples were run for approximately $3 \mathrm{~cm}$ into the gel. The entire running gel lane was excised from top to bottom and divided into three equal fractions.

Sample preparation before liquid chromatography-mass spectrometry (LC-MS/MS) measurement was performed as described in Shevchenko et al. (2006). Briefly, each gel fraction was cut into small pieces $\left(\sim 1 \mathrm{~mm}^{3}\right)$ that were washed with ammonium bicarbonate (ABC) 
buffer and then with $\mathrm{ABC}$ buffer $/ 50 \%$ acetonitrile (ACN). Subsequently, protein reduction and alkylation were performed using dithiothreitol (20 mM check) and iodoacetamide $(20 \mathrm{mM}$ check). Before in-gel digestion, gels were washed with $\mathrm{ABC}$ and $\mathrm{ACN}$ again. In-gel digestion and LC-MS/MS measurement were conducted as described before (Shevchenko et al., 2006).

\section{Protein identification, quantification and normalization}

For MaxQuant identification, an AcMNPV and an S. exigua database were downloaded from www.uniprot.org. A common contaminants database containing e.g. Trypsins (P00760, bovin and P00761, porcin) and human keratins [Keratin K22E (P35908), Keratin K1C9 (P35527), Keratin K2C1 (P04264) and Keratin K1CI (P35527)] as well as an S. exigua protein database based on Illumina HiSeq created data were included in the searches. The normal logarithm was taken from protein Label-free quantification (LFQ) MS1 intensities as obtained from MaxQuant (Bielow et al., 2016). Zero "Log LFQ" values were replaced by a value of 4.0 (somewhat lower than the lowest measured value) to make sensible ratio calculations possible. Relative protein quantitation of sample to control was done with Perseus module (available at www.MacQuant.org) by applying a two sample T-test using the "LFQ" columns obtained with false discovery rate set to 0.05 and S0 set to 1 . The nano LC-MS/MS system quality was checked with A Quality Control pipeline for Proteomics (PTXQC) using the MaxQuant result files (Bielow et al., 2016).

\section{Results}

\section{GFP-tagged PTP proteins made in larvae}

To enable GFP-tag purification of the AcMNPV PTP protein from infected larvae, four different recombinant bacmids were created, two producing the PTP protein fused to GFP either at the N- or the C-terminus (GFP-PTP and PTP-GFP), and the other two producing a catalytic mutant of the PTP protein (C119A) fused to GFP either at the N- or the C-terminus (GFPPTPM and PTPM-GFP) (Figure 1A). As a negative control an AcMNPV bacmid that lacked the $p t p$ gene and encoded non-fused GFP was used (GFP; Figure 1A). Subsequently, S. exigua larvae were infected with recombinant viruses generated with these bacmids, and proteins were purified from infected larvae at 48 hpi via a Co-IP using anti-GFP antibodies. SDS-PAGE followed by Western blot analysis using anti-GFP antibodies confirmed the presence of GFPtagged proteins in the eluted protein fractions (Figure 1B). For the GFP control sample, a clear 
band at app. $31 \mathrm{kDa}$ corresponding to the expected size of GFP appeared on the blot (Figure 1B, GFP) indicating that free GFP was produced as expected. Sharp bands at app. $50 \mathrm{kDa}$ corresponding to the various GFP-tagged PTPs were detected for the other four samples (Figure 1B, GFP-PTP, GFP-PTPM, PTP-GFP and PTPM-GFP), indicating GFP-tagged PTPs were also produced. Additionally, a band of slightly larger size than the free GFP band was observed in these samples (Figure 1B, GFP-PTP, GFP-PTPM, PTP-GFP and PTPM-GFP), most likely representing degradation products from the GFP-tagged PTP proteins. The position of GFP (Nor C-terminally of PTP) did not affect the level of production of the fusion protein. Since the samples PTP-GFP and PTPM-GFP showed less degradation products when the same amount of starting material was used, only the samples PTP-GFP and PTPM-GFP were used for the subsequent LC-MS/MS measurements.

\section{Proteomic analysis}

In the results of LC-MS/MS, unique peptides derived from GFP were detected in all samples (PTP-GFP, PTPM-GFP and GFP), and their abundance was not significantly different between the samples (Figure 2A and 2B). Peptides derived from PTP were detected in the samples PTPGFP and PTPM-GFP and their abundance was significantly higher than in the non-fused GFP control (in which PTP was absent due to the use of a ptp deletion bacmid) (Figures 2A and 2B). When comparing the sample PTP-GFP with the GFP control, eleven AcMNPV proteins and four host proteins were co-purified specifically with PTP (Table 1 and Figure 2A). When comparing the sample PTPM-GFP with the GFP control, ten other AcMNPV virus proteins and three host proteins were co-purified specifically with PTPM (Table 1 and Figure 2B). Eight AcMNPV proteins and one S. exigua protein were identified in both comparisons (Table 1). Unexpectedly, the abundance of proteins co-purified with PTP was not significantly different between the samples PTP-GFP and PTPM-GFP, despite the putative trapping abilities of the mutant (Supplementary Figure S1). Below, we describe what is known on the functions of each of the identified viral and host proteins and discuss potential links with the function of AcMNPV PTP and its role in the baculovirus infection cycle and the induction of altered host behaviour. 
A

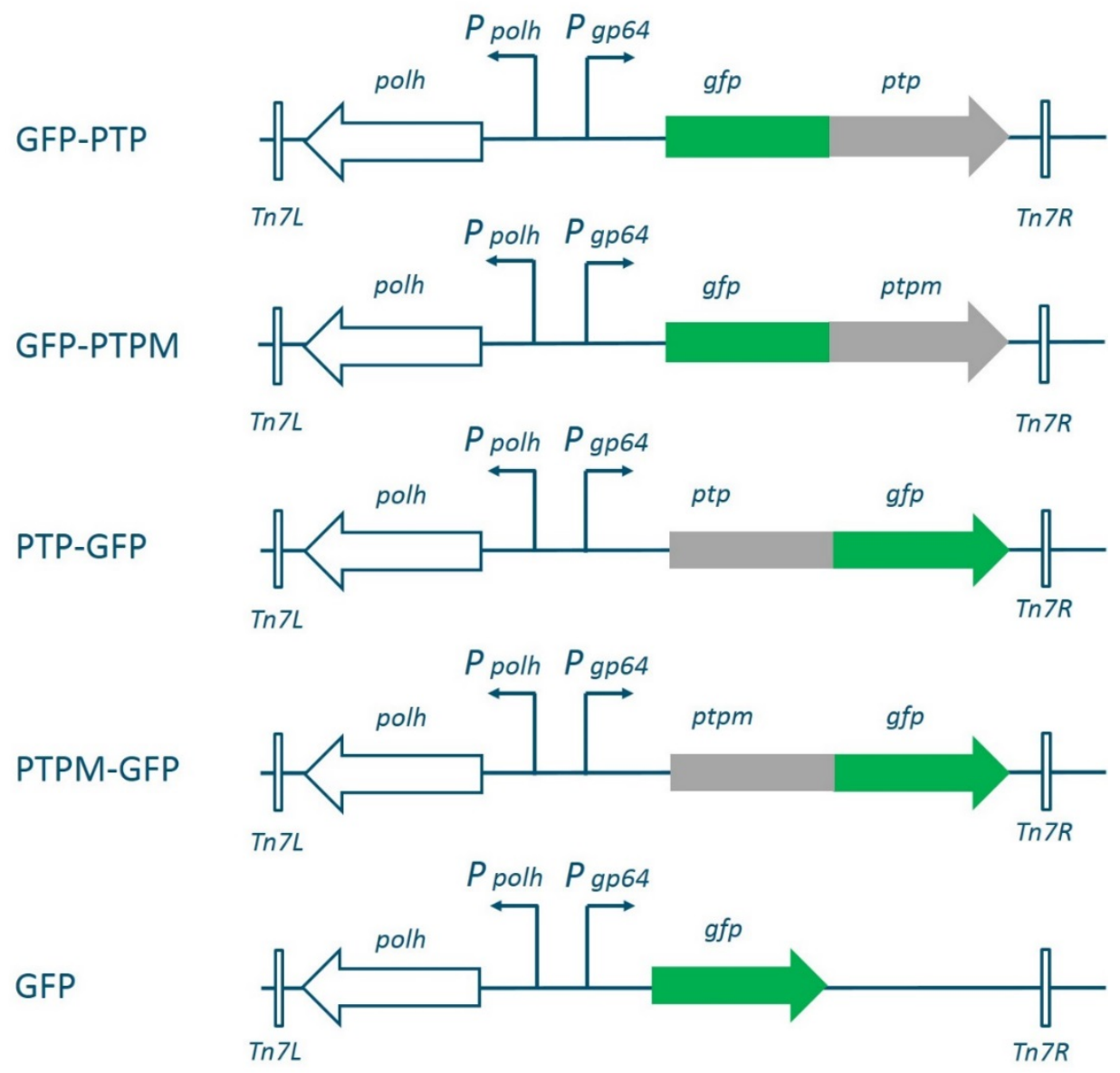

B

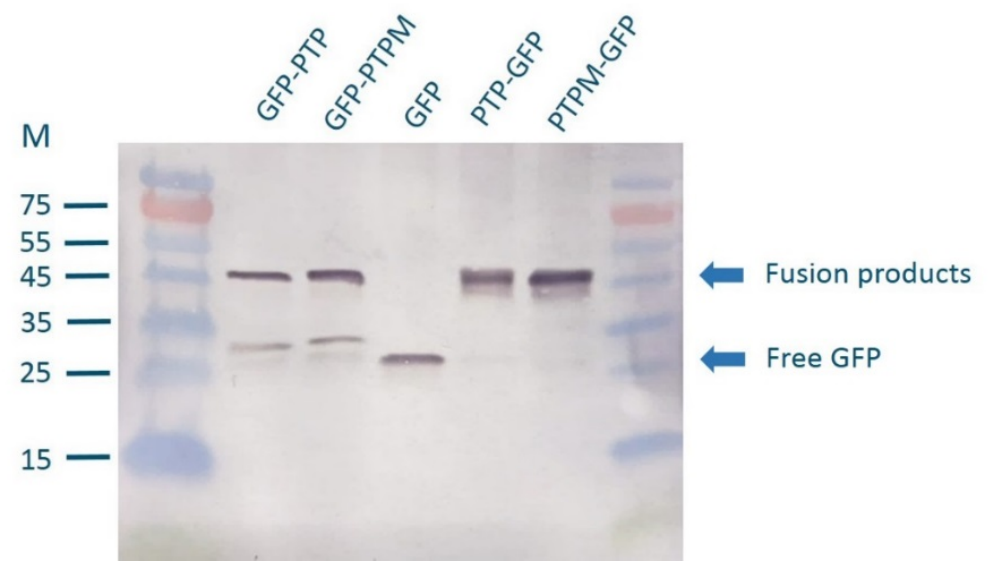

Figure 1. (A) Overview of the recombinant AcMNPV bacmids used for the substrate analysis. GFP was added to either the N- or the C-terminus of PTP and PTPM. A negative control expressing non-fused GFP was included. PTP fusion proteins and non-fused GFP were driven by the GP64 promoter $\left(\mathrm{P}_{\mathrm{gp} 64}\right)$. The polyhedrin gene (polh) and polyhedrin promoter $\left(\mathrm{P}_{\text {polh }}\right)$ were reinserted into the bacmid to enable oral infection of S. exigua larvae. (B) Western blot detection on eluted protein fractions from GFP-tag purifications of virus infected larvae. Larvae were infected with the five different viruses (see A), M = marker. 
Viral proteins involved in nucleocapsid assembly and transport that co-purified with PTP and PTPM

Several viral proteins identified in this study as interaction partners (and possible substrates) of PTP have known functions in nucleocapsid assembly (VP39, VP1054 and BV/ODV-C42) and/or progeny nucleocapsid transport (AC66, VP39, VP80, and ODV-EC27). Many of these proteins interact with infected-cell structures that play an important role in baculovirus nucleocapsid assembly, such as the virogenic stroma and nuclear filamentous-actin (F-actin). The virogenic stroma is a virus-induced nuclear structure in which the viral transcription, DNA replication and nucleocapsid assembly occurs (Kool et al., 1995; Lu and Miller, 1995; Volkman, 1988). Upon baculovirus infection actin is dramatically rearranged and forms a threedimensional nuclear network of F-actin connecting the virogenic stroma with the nuclear envelope (Volkman, 1988, 2007; Xeros, 1956). This F-actin is needed for proper nucleocapsid assembly and later on for transport of progeny nucleocapsids (Li et al., 2010; Marek et al., 2011; Volkman, 2007). Below, the order in which the identified proteins are described is based on the corresponding ORF number (lowest to highest) in the AcMNPV genome (Ayres et al., 1994). When multiple identified proteins might be involved in the same process, the potential link of AcPTP with these proteins is discussed together.

VP1054 (ORF54) is encoded by a baculovirus core gene and is a minor capsid protein present in both BVs and ODVs (Wu et al., 2008). VP1054 is required for nucleocapsid assembly. More precisely, VP1054 plays an important role in transporting the capsid protein VP39 to the nucleocapsid assembly site. Deletion of VP1054 leads to the formation of abnormally elongated capsid structures and to reallocation of VP39 to the periphery of the nucleus instead of to the virogenic stroma (Guan et al., 2016). Additionally, VP1054 functions as a virally encoded PUR $\alpha$-like protein (a DNA- and RNA-binding protein with specificity for GGN repeats) and is required for proper viral DNA encapsidation (Marek et al., 2013).

BV/ODV-C42 (ORF101) is a protein of $42 \mathrm{kDa}$ in size and encoded by a gene transcribed in the late stage of infection, see review by Cohen et al. (2009). Homologues of $b v / o d v-c 42$ gene are present in all sequenced baculoviruses except for CuniNPV (Slack and Arif, 2007). The encoded protein is a component of the nucleocapsids of both BVs and ODVs (Braunagel et al., 2001). Deletion of this gene (ac101) from the AcMNPV bacmid does not affect viral genome replication, but infectious particles are no longer produced. Only immature nucleocapsids are formed (Vanarsdall et al., 2007), suggesting a crucial role for BV/ODV-C42 in nucleocapsid 
assembly. BV/ODV-C42 is known to play a crucial role in the re-organisation of F-actin in infected cells. Previous studies have shown that nuclear F-actin polymerization is triggered by the viral capsid protein P78/83 (Charlton and Volkman, 1993; Volkman, 2007). The actinrelated protein 2/3 (ARP2/3) complex is required to activate P78/83. BV/ODV-C42 has a putative nuclear localisation signal (NLS) and has been shown to recruit and transport p78/83 from the cytoplasm to the nucleus, where then actin polymerization is initiated (Braunagel et al., 2001; Li et al., 2010). Besides recruiting P78/83 to the nucleus, BV/ODV-C42 also appears to play an active role in nuclear F-actin formation itself. The putative pocket protein binding sequence (PPBS) motif of BV/ODV-C42 is essential for exerting its function in nuclear actin polymerization (Li et al., 2010).

The fact that VP1054 and BV/ODV-42, which have both important roles in nucleocapsid assembly, are co-purified with AcPTP implies that AcPTP may play a role in nucleocapsid assembly.

AC66 (ORF 66) is a structural protein of the ODV and BV nucleocapsids (Ke et al., 2008; Wang et al., 2010). Ac66 is conserved among all sequenced lepidopteran baculovirus genomes and encodes a $94 \mathrm{kDa}$ protein. AC66 contains an N-terminal desmoplakin domain and a CorAlike $\mathrm{Mg}^{2+}$-transporter region. Desmoplakin is one of the major components of desmosomes, which are cell structures specialized in cell-to-cell adhesion. The CorA-like Mg2+-transporter region indicates that AC66 may also be involved in transportation of metal ions (Kool et al., 1995). Deletion of ac66 reduced BV yields by $99 \%$ due to insufficient transport of nucleocapsids from the nucleus to the cytoplasm. More specifically, there was a strongly reduced release of nucleocapsids from the virogenic stroma (Ke et al., 2008). On the other hand, deletion of ac66 did not affect viral DNA replication and nucleocapsid assembly. Ac66 is also involved in ODV and OB formation: OB formation and ODV virion synthesis were interrupted in a deletion mutant (Ke et al., 2008).

The major capsid protein VP39 (ORF89) is conserved among all sequenced baculoviruses and the most abundant structural protein of the nucleocapsids (Charlton and Volkman, 1993). During virus assembly, VP39 monomers are arranged in stacked rings around the nucleoprotein core. VP39 also participates in the rearrangement and polymerization of host nuclear actin (Charlton and Volkman, 1993). During the later stage of infection, VP39 co-localizes with peripheral microtubules in the nucleus of infected cells as shown in a confocal microscopy study of AcMNPV-infected Trichoplusia ni cells (Danquah et al., 2012). Moreover, when 
microtubule formation was inhibited with colchicine- BV production was reduced. Furthermore, VP39 interacts with the tetratricopeptide repeat domain of kinesin (a protein moving along the microtubules) and probably this interaction enforces the transportation of nucleocapsids (Danquah et al., 2012). These data indicate that microtubules and potentially also kinesin are involved in anterograde trafficking of baculoviruses.

The vp80 gene (ORF 104) encodes a $80 \mathrm{kDa}$ protein. Homologues of the $v p 80$ gene are only found in alphabaculoviruses. The encoded VP80 is an essential component of both BV and ODV particles. VP80 is an end-linked nucleocapsid protein, which is normally associated with one end of the nucleocapsids. Furthermore, VP80 interacts with the viral genomic DNA via a basic helix-loop-helix domain at the C-terminus (Marek et al., 2012). Deletion of $v p 80$ prevented migration of nucleocapsids from the virogenic stroma to the nuclear periphery (Marek et al., 2011). As indicated above, host F-actin is dramatically rearranged during infection, forming a three-dimensional network connecting the virogenic stroma and nuclear envelope (Li et al., 2010; Marek et al., 2011; Volkman, 2007). VP80 physically interacts with this nuclear F-actin and plays an important role in progeny nucleocapsid egress from the virogenic stroma to the nuclear periphery (Marek et al., 2011).

Interestingly, it is predicted that VP80 functions as a paramyosin protein, which is a major structural component of muscular thick filaments in insects (Marek et al., 2011). Paramyosins need to interact with actin-based (thin) filaments to convert chemical energy (ATP) into mechanical force (movement), which would be needed to transport nucleocapsids from virogenic stroma to the nuclear periphery. Furthermore, the above mentioned AC66 possesses a functional domain homologous to the actin-binding domain of myosin VI (Volkman, 2007). It is therefore hypothesized that VP80 and AC66 may work in concert with myosin to transport nucleocapsids to the nuclear periphery.

ODV/EC27 (ORF144) is another structural protein (33.5 $\mathrm{kDa}$ in size and encoded late in the infection) encoded by a baculovirus core gene. It has been found that ODV/EC27 localized to the ODV envelope and capsid (Braunagel et al., 1996). ODV/EC27 contains a cyclin-like domain, suggesting a function for ODV/EC27 in cell cycle control (Belyavskyi et al., 1998). Experimental data showed that ODV/EC27 associates with either cellular cyclin CDC2 or CDK6 to form a complex (Belyavskyi et al., 1998). The cdc2-EC27 complex exhibits a cyclin B-like function, which arrests the host cell cycle at the G2/M phase to optimize viral gene transcription. The complex accumulates in the nucleus and phosphorylates nuclear lamins 
(Belyavskyi et al., 1998). Phosphorylation of nuclear lamins results in breakdown of the nuclear lamin, thus increasing the envelope fluidity and this might eventually enhance progeny virus release from the nucleus. The alternative cdk6-EC27 complex presents a cyclin D-like function that elongates the cell replication cycle. Cdk6-EC27 also interacts with viral proliferating cell nuclear antigen (PCNA) and forms a complex that phosphorylates host histone H1, which is hypothesized to support viral replication (Belyavskyi et al., 1998).

During transport of baculovirus nucleocapsids from nucleus to cytoplasm, a wide range of molecular pathways are involved. Phosphorylation of nuclear lamins is important in this process, as detailed above. However, whether this process is balanced by dephosphorylating enzymes such as PTP, is still largely unknown. The co-purification of AcPTP with AC66, VP39, VP80, and ODV-EC27 suggest that PTP may have an important function in several steps in the transport of progeny virus.

\section{Interaction of PTP $(M)$ with host proteins with potential functions in nucleocapsid transport}

Two host proteins with potentially important roles in nucleocapsid transport were also copurified with $\operatorname{AcPTP(M):~Lamins~} \mathbf{C}$ and myosin regulatory light chain 2 . The known information about these two proteins in nucleocapsid transport is listed below.

Lamins are important structural proteins of eukaryotic cells. Lamins line the inside of the nuclear membrane and provide mechanical stability. They are also involved in DNA replication, transcription and chromatin organization (Dechat et al., 2010). Lamins are type V intermediate filament proteins and are categorized into two groups: Type-A lamins include lamin A and C. B-type lamins include lamin B1, lamin B2 and lamin B3 (Zhang et al., 2017). To bud out from the nuclear membrane of infected cells in order to travel to the cytoplasm to become BVs, baculovirus nucleocapsids have to bypass the physical barrier formed by the nuclear lamins underlying the nuclear membrane. Previous research showed that the phosphorylation level of lamin B increased upon AcMNPV infection and that part of the nuclear lamins were redistributed. Co-purification of AcPTP with Lamin-C further supports a role of PTP in progeny nucleocapsid export from the nucleus.

Myosin regulatory light chain 2 (RLC) is also known as the regulatory light chain of myosin (RLC). Basically, RLC regulates myosin motility and functions via interacting with the neck/tail region of myosin (Rayment et al., 1993). A study in mammalian cells suggested that RLC is required to maintain the stability of myosin II, which is needed for cellular integrity (Park et al., 2011). In insects, the exact function of RLC is not reported yet, however, it is likely 
that it exerts a similar function as in mammalian cells. Like F-actin, myosin has been suggested to be involved in mediating the transport of nucleocapsids from the virogenic stroma towards the nuclear periphery, or even further to the cell periphery (Volkman, 2007). Therefore, RLC might have a function in this process as well.

The apparent association between AcMNPV PTP with structural viral proteins and host proteins involved in nucleocapsid transportation implies that PTP plays an, as yet unknown, function in transporting nucleocapsids from the virogenic stroma to the nuclear periphery or even further to the cytoplasm.

\section{Viral proteins involved in $B V$ and $O D V$ envelopment that co-purified with $P T P(M)$}

Three viral proteins involved in BV and ODV envelopment co-purified specifically with PTPM. Below the known information about these viral proteins is described.

Ac109 (ORF109) is a baculovirus core gene, conserved among all sequenced baculovirus genomes, and is expressed during the late phase of the infection cycle. AC109 is present within or associated with ODVs (Lehiy et al., 2013). Deletion of ac109 did not affect viral genome replication, late gene synthesis or polyhedrin production in virus infected cells, however, cell to cell virus transmission was absent, indicating that no BVs, or at least no infectious BVs, were produced. Deletion of ac 109 also blocked membrane envelope formation of nucleocapsids prior to occlusion, although nucleocapsids localized along the ring zone of the nucleus. Therefore, ac109 may play a role in the production of infectious BVs and in the envelopment of nucleocapsids into ODV particles in the nucleus (Lehiy et al., 2013).

Ac132 (ORF132) homologues are present in the genomes of all group I NPVs. AC132 associates with nucleocapsids of both BVs and ODVs (Yang et al., 2014). During infection, $\mathrm{AC} 132$ forms a ring zone at the periphery of the nucleus at $24 \mathrm{hpi}$. Deletion of ac132 did not affect viral DNA replication, however, the infection was limited to a single cell. In ac132 mutant virus-infected cells, the synthesis of nucleocapsids, ODVs and OBs still occurred, however, virogenic stroma and $\mathrm{OB}$ formation were delayed. These data indicate that $\mathrm{AC} 132$ plays a role in nucleocapsid envelopment (Yang et al., 2014).

Ac142 (49K, ORF142) is also a core gene and predicted to encode a 477-aa protein with a transmembrane domain. AC142 is associated with the nucleocapsids of both BVs and ODVs. Deletion of $a c 142$ restricted the infection to single cells as no infectious BVs were produced. Ac142 was not needed for viral DNA replication. During ODV production, AC142 was not 
needed for nucleocapsid formation, but was required for subsequent ODV envelopment and embedding of virions into OBs. Therefore, AC142 is required for both BV and ODV production (McCarthy et al., 2008).

The above-mentioned viral proteins all are involved in BV and/or ODV budding/envelopment. The fact that PTP is co-purified with these proteins indicates that PTP may have function in these processes.

\section{Viral and host proteins potentially involved in inducing apoptosis}

Though AcPTP has not been reported in inducing apoptosis in infected host cell, in our study, two proteins that play a role in inducing apoptosis were also co-purified with AcPTP. The known information about these two proteins were listed here.

Immediately early gene-1 (ORF147): The iel gene is conserved among all sequenced lepidopteran NPVs. The 582-aa long protein contains an acidic activation domain, a DNA binding domain and an oligomerization domain (Schultz et al., 2009). IE1 is a potent transcriptional transactivator for baculovirus early genes, and as such it is required for virus DNA replication, and for BV and ODV production (Leisy et al., 1995; Nagamine et al., 2005). IE1 is synthesized very early during infection and accumulates in the nucleus. Its expression is maintained during the course of infection (Pullen and Friesen, 1995). IE1 expression is a trigger for apoptosis, but this is normally counteracted by the AcMNPV P35 protein, which is a strong apoptosis inhibitor (Schultz et al., 2009). An AcMNPV p35 deletion mutant caused widespread apoptosis in Sf21 cells. Apoptosis induction was abolished when iel transcripts were silenced during infection with the AcMNPV p35 deletion mutant. It was shown that AcMNPV IE1 is indirectly involved in the formation of apoptotic bodies in virus-infected Sf21 cells by initiating virus DNA replication events that subsequently trigger cell death (Schultz et al., 2009).

Histone H2B.3: Histones are highly conserved structural proteins in eukaryotic nucleosomes. Histone H2B.3 is a component of the nucleosome subunit H2B. Histones have important functions in packaging and maintaining of chromosomes, regulating transcription and DNA replication and repair (McGinty et al., 2008; Vanfleteren et al., 1986). During baculovirus replication, host DNA replication is arrested, while viral DNA is supported (Nguyen et al., 2013). Moreover, virus infection causes activation of several phosphoinositide 3- kinases, which subsequently phosphorylate Histone 2A, eventually leading to cell cycle arrest or apoptosis. As a subunit of Histone 2B, the phosphorylation status of Histone H2B.3 may also have a crucial role in regulating apoptosis. Co-purification of AcPTP and Histone 2B suggests 
that PTP may have a role in balancing phosphorylation levels of histone proteins, which may eventually contribute to induction of apoptosis in infected cells (Nguyen et al., 2013).

IE1, and possibly the phosphorylation status of Histone H2B.3, are involved in inducing apoptosis during baculovirus infection. Therefore, AcPTP may play a role in regulating apoptosis in infected hosts.

\section{Copurification of host ribosomal proteins}

$40 S$ ribosomal protein $S 27$ a and $40 S$ ribosomal protein $S 9$ are both structural components of the small (40S) ribosomal subunit. These two proteins, like other ribosomal proteins, are highly conserved and abundantly expressed. Ribosomal protein S27a is derived from a fusion protein consisting of ubiquitin at the $\mathrm{N}$-terminus and ribosomal protein S27 at the C-terminus (Lindström and Nistér, 2010; Sun et al., 2011). Co-purification of PTP with these two ribosomal proteins suggests that PTP may play an unknown function in viral protein translation. However, the co-purification of PTP and ribosomal proteins may also simply result from a-specific binding between these proteins due to the high abundance of ribosomal proteins (even though the amount was significantly lower in the control sample) (Redman and Rechsteiner, 1989). Ribosomal proteins have been reported as one of the most abundant co-purified protein in many studies, including proteomic studies of baculoviruses. In the proteomic study of Sf9 cells infected with AcMNPV, ribosomal protein S9 was the most abundant host protein co-purified with AcMNPV (Carinhas et al., 2011). The same results were also found in a proteomic study of baculovirus Anticarsia gemmatalis MNPV ODVs (Braconi et al., 2014). Future experiments are needed to prove this.

\section{Proteins with unknown functions}

Several proteins were co-purified specifically with AcPTP, but for most of these proteins information on their function is lacking.

Ac18 (DA41/ORF18) is conserved among alpha- and betabaculoviruses (Cohen et al., 2009). A previous study showed that AC18 was not needed for nucleocapsid formation, nor for BV and ODV production, as cells infected with an ac18 deletion virus showed similar phenotypes as cells infected with the WT virus (Wang et al., 2007). Spontaneous deletion of ac18 occured in serial passages of a virus stock in cell culture. Deletion of ac18 did not reduce virus infectivity for $T$. $n i$ larvae in a dose-dependent assay, but it took longer for the mutant virus to kill the host than for the WT virus (Wang et al., 2007). Therefore, it is hypothesized that AC18 
is important for an efficient infection, which could indirectly contribute to a higher virus transmission rate.

Ac51 (Baculovirus $\mathbf{J}$ domain protein) is found in all alphabaculoviruses. The encoded protein contains a coiled-coil domain and an RNA recognition motif and is present in both BVs and ODVs (Wang et al., 2002). The orthologue from Spodoptera litura NPV is a late gene and encodes a protein described as baculovirus $\mathrm{J}$ domain protein (BJDP). BJDP in E. coli has chaperone activity and is involved in folding of other proteins (Langer et al., 1992). The function of BJDP in baculoviruses is not fully understood.

Ac58/59 (ORF58/59) is actually a single ORF that encodes a protein of 172 amino acids. AC58/59 associates with both BVs and ODVs. However, ac58/59 seems not essential for virus replication, since deletion of the ac58/59 homologues in BmNPV does not affect virus production and virus infectivity (Ono et al., 2012). AC58/59 has a ChaB domain, indicative for a role in cation transportation. However, the exact function of AC58/59 is not known (Cohen et al., 2009).

Though the functions of AC18, BJDP and AC58/59 of AcMNPV are not clear, the copurification of these proteins with PTP suggests that PTP might be involved in other, currently unknown processes.

\section{Co-purified proteins from other viruses}

Interestingly, proteins from two other viruses were also co-purified with AcPTP, including the DNA metabolism protein from Heliothis virescens ascovirus $3 g$ (HvAV-3g) and the helicase and RNA-dependent RNA-polymerase (RdRp) from Spodoptera exigua iflavirus 1 (SeIV1).

HvAV-3g is a double stranded DNA virus classified in the family Ascoviridae, that has been detected in S. exigua before (Li et al., 2016). Ascoviruses in general have a low infection rate and ascovirus transmission is dependent on parasitoids. Infected hosts lack apparent infection symptoms (Li et al., 2016). The function of the HvAV-3g DNA metabolism protein is unknown, however, its homologue in eukaryotes seems to be involved in chromosome condensation, segregation, cohesion and DNA recombination repair (Graumann, 2001).

Peptides derived from the helicase and RdRp of SeIV1 (family Iflaviridae) were also copurified with PTP. SeIV1 has a single stranded RNA genome. The genome is translated into a polyprotein, which is subsequently cleaved into structural and non-structural proteins [see review by van Oers (2010)]. Like HvAV-3g, SeIV1 can infect $S$. exigua, but in general has low 
virulence (Carballo et al., 2017). Artificial co-infection of S. exigua larvae with SeIV1 and the baculovirus SeMNPV led to a decrease in SeMNPV OB yield (Carballo et al., 2017).

A

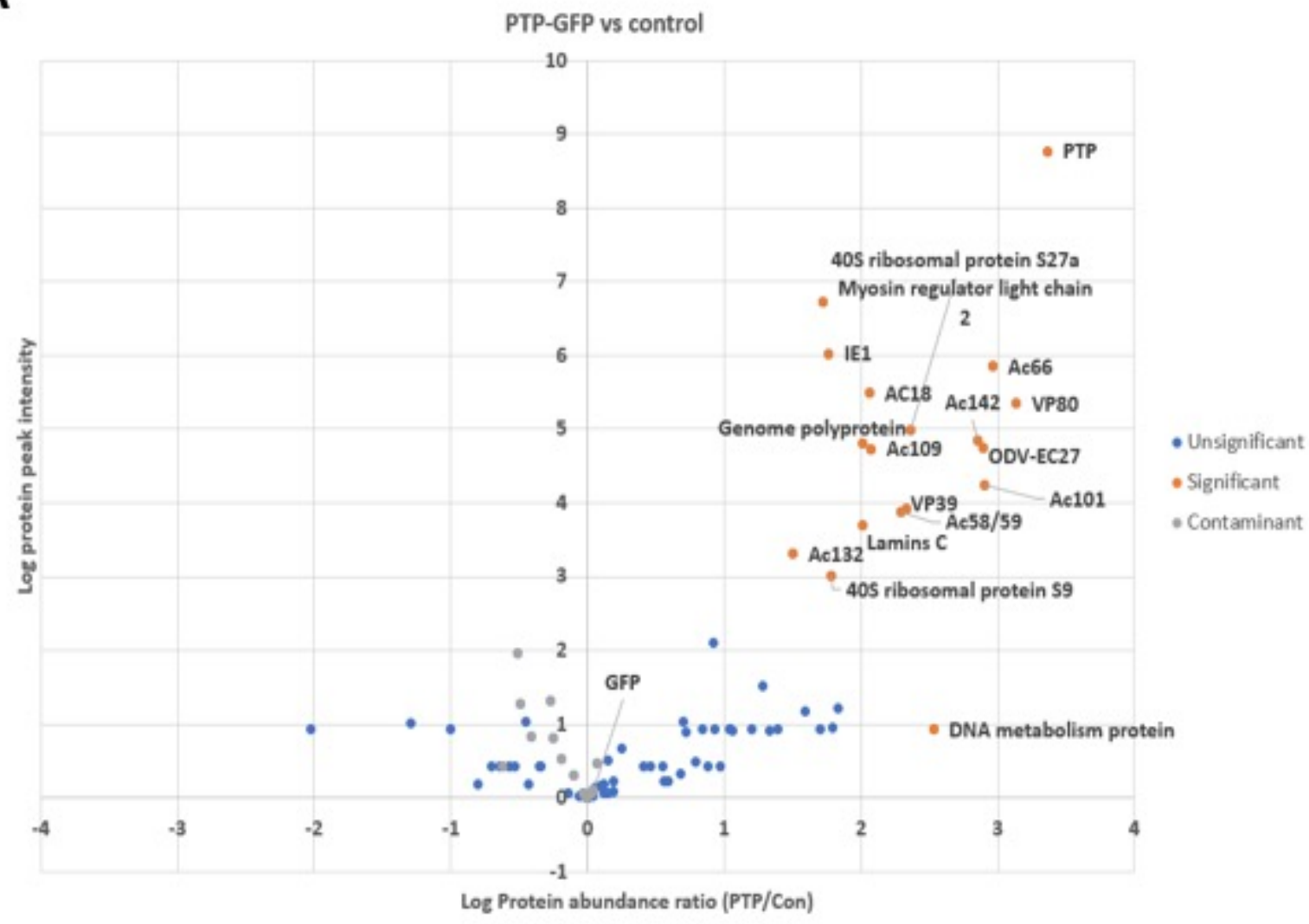

B

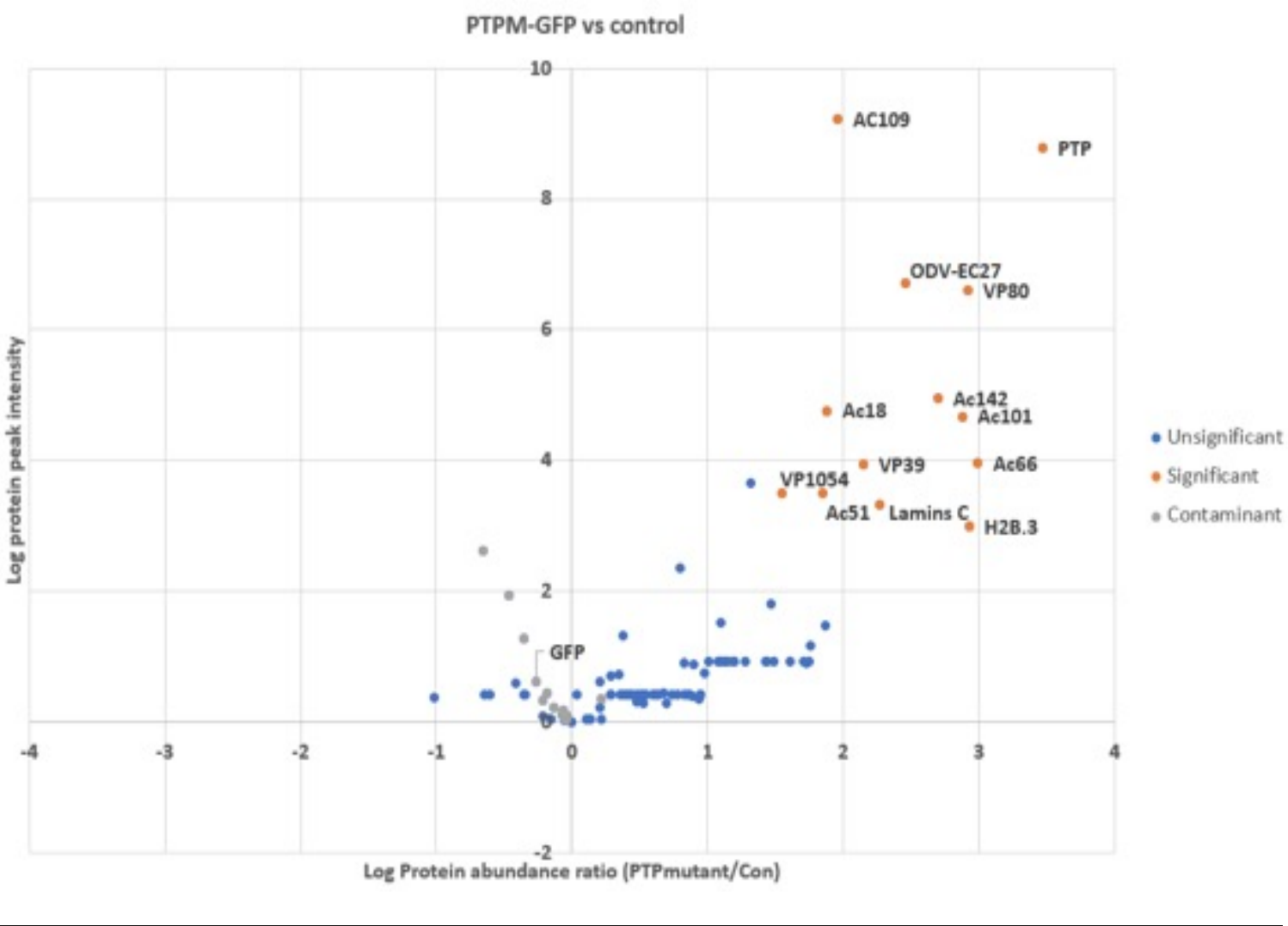


Figure 2. Mass spectrometric proteomic analysis of purified PTP-GFP proteins (A) and PTPM-GFP proteins (B). PTP-GFP and PTPM-GFP proteins were prepared from AcMNPV infected larvae and precipitated by GFP antiserum. The precipitation experiments were conducted three times from the same starting material, precipitated proteins were analysed with LC-MS/MS analysis using MaxQuant software and the statistical analysis was performed with the Perseus module. The Y-axis shows the relative abundance (peak height) as $\log (10)$ values, and X-axis shows the $\log (10)$ of the abundance ratio of proteins precipitated in the PTP-GFP group over proteins precipitated in the GFP control group. Proteins that were significantly enriched in the PTP(M)-GFP sample over the GFP control are shown in orange $(\mathrm{P}<0.01)$. Proteins that are not significantly enriched are shown in blue. Contaminants such as keratins and trypsin are shown in grey.

\section{Discussion}

Phosphorylation and dephosphorylation of proteins are common regulation mechanisms that control the function of proteins and allow proteins to rapidly switch between different activity states. There is no doubt that phosphorylation and dephosphorylation play important roles during baculovirus infection (Cheley et al., 1992; Funk and Consigli, 1993; Vialard and Richardson, 1993). Our analysis of possible target proteins that co-purified with AcMNPV PTP revealed several host and viral proteins that may serve as a substrate to the AcMNPV PTP enzyme. Previous studies showed that baculovirus PTP is associated with both BVs and ODVs and is able to remove phosphate residues from both protein and RNA substrates (Li and Guarino, 2008).

In our study, the identified proteins that co-purified with AcPTP are involved in different steps of the baculovirus replication cycle, including, but probably not limited to nucleocapsid assembly, nucleocapsid transportation, ODV envelopment and release of mature virus particles to enhance virus transmission.

The co-purified proteins VP39, VP1054 and BV/ODV-42 have important functions in baculovirus assembly. Site-specific dephosphorylation of baculovirus proteins is known to play a significant role during baculovirus assembly. For example, AcMNPV P6.9 (encoded by the core gene Ac100) is needed for proper virus genome encapsidation and virus assembly. Multiple versions of phosphorylated P6.9 are detected in infected cells, however, only the unphosphorylated version is detected in BVs (Lai et al., 2018). When the dephosphorylated sites of P6.9 are mutated to glutamic acids (phosphorylation-mimic mutant), nucleocapsid assembly is interrupted. Therefore, dephosphorylation of specific sites of P6.9 is essential for virus genome encapsidation and virus assembly (Lai et al., 2018). Similar results were also 
found for the P6.9 homologue VP12 from Plodia interpunctella granulosis virus (PiGV): the VP12 protein is needed for the release of viral DNA from nucleocapsids at the beginning of the infection cycle and dephosphorylation of VP12 allows packaging of progeny DNA molecules

Table 1: Baculovirus (AcMNPV) proteins and host proteins that were co-purified with the AcMNPV PTP protein. MS/MS spectra were searched against the Uniprot database and the $S$. exigua RNA-seq database.

\begin{tabular}{|c|c|c|c|}
\hline Protein name & Origin & Description & Co-purified with \\
\hline $\mathrm{BV} / \mathrm{ODV}-\mathrm{C} 42$ & AcMNPV & $\begin{array}{l}\text { ORF 101, involved in F-actin polymerization and } \\
\text { nucleocapsid assembly }\end{array}$ & PTP-GFP and PTPM-GFP \\
\hline VP39 & AcMNPV & $\begin{array}{l}\text { ORF } 89 \text {, involved in nucleocapsid assembly and } \\
\text { transportation from nucleus to cytoplasm }\end{array}$ & PTP-GFP and PTPM-GFP \\
\hline VP1054 & AcMNPV & ORF 54, involved in nucleocapsid assembly and maturation & PTPM-GFP \\
\hline Ac66 & AcMNPV & $\begin{array}{l}\text { ORF } 66 \text {, involved in transporting nucleocapsids from } \\
\text { nucleus to cytoplasm }\end{array}$ & PTP-GFP and PTPM-GFP \\
\hline VP80 & AcMNPV & $\begin{array}{l}\text { ORF } 104 \text {, involved in transporting nucleocapsids from } \\
\text { nucleus to cytoplasm }\end{array}$ & PTP-GFP and PTPM-GFP \\
\hline AC109 & AcMNPV & $\begin{array}{l}\text { ORF 109, involved in BV production and ODV } \\
\text { envelopment }\end{array}$ & PTP-GFP and PTPM-GFP \\
\hline AC142 & AcMNPV & $\begin{array}{l}\text { ORF 142, involved in BV production and ODV } \\
\text { envelopment }\end{array}$ & PTP-GFP and PTPM-GFP \\
\hline AC132 & AcMNPV & $\begin{array}{l}\text { ORF 132, involved in BV production and nucleocapsid } \\
\text { envelopment }\end{array}$ & PTP-GFP \\
\hline DA41/Ac18 & AcMNPV & ORF 18 , needed for efficient virus infection in host & PTP-GFP and PTPM-GFP \\
\hline IE1 & AcMNPV & $\begin{array}{l}\text { ORF 147, activation for other transcripts and inducing } \\
\text { apoptosis }\end{array}$ & PTP-GFP \\
\hline ODV/EC27 & AcMNPV & $\begin{array}{l}\text { ORF144, involved in regulate host cell cycle and possibly } \\
\text { involved in enhancing virus dissemination }\end{array}$ & PTP-GFP and PTPM-GFP \\
\hline AC58/59 & AcMNPV & ORF 58 , function unknown & PTP-GFP \\
\hline BJDP & AcMNPV & ORF 51, function unknown & PTPM-GFP \\
\hline $\begin{array}{l}\text { DNA metabolism } \\
\text { protein }\end{array}$ & HvAV-3g & Potentially play an important role in virus DNA replication & PTP-GFP \\
\hline Helicase & SeIV1 & Unwind replication template & PTP-GFP \\
\hline $\operatorname{RdRp}$ & SeIV1 & Catalysis synthesis of new viral genome & PTP-GFP \\
\hline Lamin-C & S. exigua & $\begin{array}{l}\text { Nucleus structure protein, involved in protein and chromatin } \\
\text { binding and providing mechanical stability }\end{array}$ & PTP-GFP and PTPM-GFP \\
\hline $\begin{array}{l}\text { Myosin regulatory } \\
\text { light chain } 2\end{array}$ & S. exigua & $\begin{array}{l}\text { Regulating myosin mobility, might be involved in transport } \\
\text { of nucleocapsids from the virogenic stroma to the cytoplasm }\end{array}$ & PTPM-GFP \\
\hline Histone H2B.3 & S. exigua & $\begin{array}{l}\text { Structural component of nucleosomes, might play a role in } \\
\text { enhancing virus transmission }\end{array}$ & PTPM-GFP \\
\hline
\end{tabular}




$\begin{array}{llll}\text { 40S ribosomal } & \text { S. exigua } & \text { Structural component of host ribosome } & \text { PTPM-GFP } \\ \text { protein S27a } & & \\ \text { 40S ribosomal } & \text { S. exigua } & \text { Structural component of host ribosome } & \text { PTPM-GFP } \\ \text { protein S9 } & & & \end{array}$

into virions at the end of infection cycle (Funk and Consigli, 1993). Dephosphorylation of P6.9 is mediated by another baculovirus protein, $38 \mathrm{~K}$ (encoded by ORF98). $38 \mathrm{~K}$ functions as a haloacid dehologenase, which is involved in phosphoryl transfer (Lai et al., 2018). VP1054 is an interacting partner of $38 \mathrm{~K}$ (in a yeast two hybrid studies) and also involved in DNA encapsidation. Therefore, AcPTP might affect baculovirus nucleocapsid assembly by dephosphorylating VP1054 and other structural viral proteins.

As mentioned above, BmNPV PTP is involved in inducing hyperactivity in B. mori larvae, however the phosphatase activity of PTP was not needed for this process in this insect-virus combination (Kamita et al., 2005; Katsuma, 2015; Katsuma et al., 2012), which may suggest that BmPTP, at least in the induction of hyperactivity, functions as a structural protein. For AcMNPV, the phosphatase activity of PTP is, however, required to induce hyperactivity in $S$. exigua larvae (van Houte et al., 2012). Interestingly, BmPTP interacted with the baculovirus phosphoprotein 78/83 (PP78/83) in a yeast-2-hybrid assay (Katsuma et al., 2012). The interaction between these two proteins supports the conclusion that BmPTP functions as a BVassociated structural protein rather than an enzyme to induce hyperactivity. In our study, AcPTP did not co-purify with PP78/83, however, the co-purified proteins BV/ODV-C42 and VP1054 have been reported to interact with PP78/83 (Guan et al., 2016; Li et al., 2010). Whether the (indirect) interaction of PTP with PP78/83 has a role in PTP-induced caterpillar hyperactivity in general is currently unclear. Though we did not investigate the interaction between AcPTP and RNA in this study, it is possible that AcPTP induces hyperactivity by affecting host RNA substrates.

IE1 and Histone H2B.3 may be involved in triggering apoptosis in the infected host. Commonly, apoptosis induction is an effective host response to limit virus infection in the initial phase (Clem, 2001, 2005). As a countermeasure, baculoviruses encode various proteins which have anti-apoptotic abilities (Clem, 2001, 2005). Moreover, some baculoviruses act as inducers of apoptosis in specific host cells, e.g. in hemocytes to down regulate immune responses (Han et al., 2018; Chapter 5). Our previous research showed that the ptp2 gene from SeMNPV, encoding a protein that belongs to the same PTP protein family as AcMNPV PTP, is involved 
in inducing apoptosis. Although the similarity between PTP and PTP2 is very low (34\% aa similarity), co-purification of PTP with IE1 and Histone H2B.3 may indicate that PTP is involved in regulating apoptosis during baculovirus infection.

Both the DNA metabolism protein of the ascovirus HvAV-3g and the helicase and RdRp of the iflavirus SeIV1 potentially play an important role in genome replication of the respective viruses. A phosphatase gene is absent in both viral genomes. Potentially, interactions between viruses may exist during co-infection and the genome replication of one virus might be supported by proteins from another virus. However, to what extend AcMNPV PTP affects HvAV-3g and SeIV1 replication needs further investigation.

Three limitations of our study should be considered when interpreting the data. Firstly, the proteomic data are only generated from one time point after infection and cannot reflect the dynamic changes of AcPTP substrates over time. To understand how the substrates of AcPTP are changing over time, samples at different time points after infection should be collected and compared. Secondly, the whole larva was used for sample preparation. Therefore, substrates of AcPTP originating from different tissues might be mixed and substrates with low abundancy could be missed. Searching for PTP-interacting (host) proteins in specific tissues would be an exciting next step to take. Thirdly, in our study, only five host proteins that co-purified with AcPTP were identified from the protein database. Since the genome of S. exigua has not been sequenced, a de novo transcriptome assembly derived from RNA-seq data of S. exigua larvae was used as the database for protein identification. However, other important interacting proteins may not be discovered due to the lack of a properly annotated genome.

Parasites have been reported to achieve host behavioural manipulation via invading the host's central nervous system (CNS) (Lefevre et al., 2009). In BmPTP-induced hyperactivity in $B$. mori larvae, a mutant virus lacking the $p t p$ gene showed a significantly reduced infectivity of larval brain tissues (Katsuma et al., 2012). It is hypothesized by these authors that virus infection in the brain leads to an excitatory state which is needed for inducing hyperactivity. The same may apply for AcPTP-induced hyperactivity in S. exigua larvae: AcMNPV may induce hyperactivity in the host by invading the host CNS and ptp may play an important role in this process. The fact that we co-purified lamins may point in this direction. Apart from being important for remodeling the nuclear architecture to facilitate egress of nucleocapsids from the nucleus, as described in the results, here we hypothesize that this interaction with lamins may allow the virus to invade the larval brain. Lamins play curcial roles in brain development and 
defects in lamins cause an array of (mental) disorders in humans and rats (Schulze et al., 2009; Young et al., 2012). Lamins play essential roles in neuronal migration in the developing brain and in neuronal survival (Jung et al., 2013; Schulze et al., 2009; Young et al., 2012).

In conclusion, we performed a proteomic study to identify viral/host proteins that interact with AcPTP. Several of the identified proteins are known to be important for nucleocapsid assembly, nucleocapsid transport, ODV envelopment and/or virus dissemination, suggesting that AcPTP functions both as a structural protein and as a phosphatase enzyme. Some of these proteins may potentially be involved in PTP-mediated hyperactivity of infected larvae. We hypothesize that AcPTP may control virus entry into certain host tissues, for example the host brain, ultimately leading to the behavioural changes observed in the infected hosts.

\section{Acknowledgements}

The authors thank Stineke van Houte for initiating this study. Els Roode and Hanke Bloksma are acknowledged for rearing Spodoptera exigua larvae. Mark Roosjen is sincerely thanked for the instruction of the GFP-tag protein purification. Gerben Drees, Amy Clarijs and Ioana Nicorescu are thanked for their assistance in making recombinant bacmids. 


\section{Supplementary Material}

PTP-GFP vs PTPM-GFP

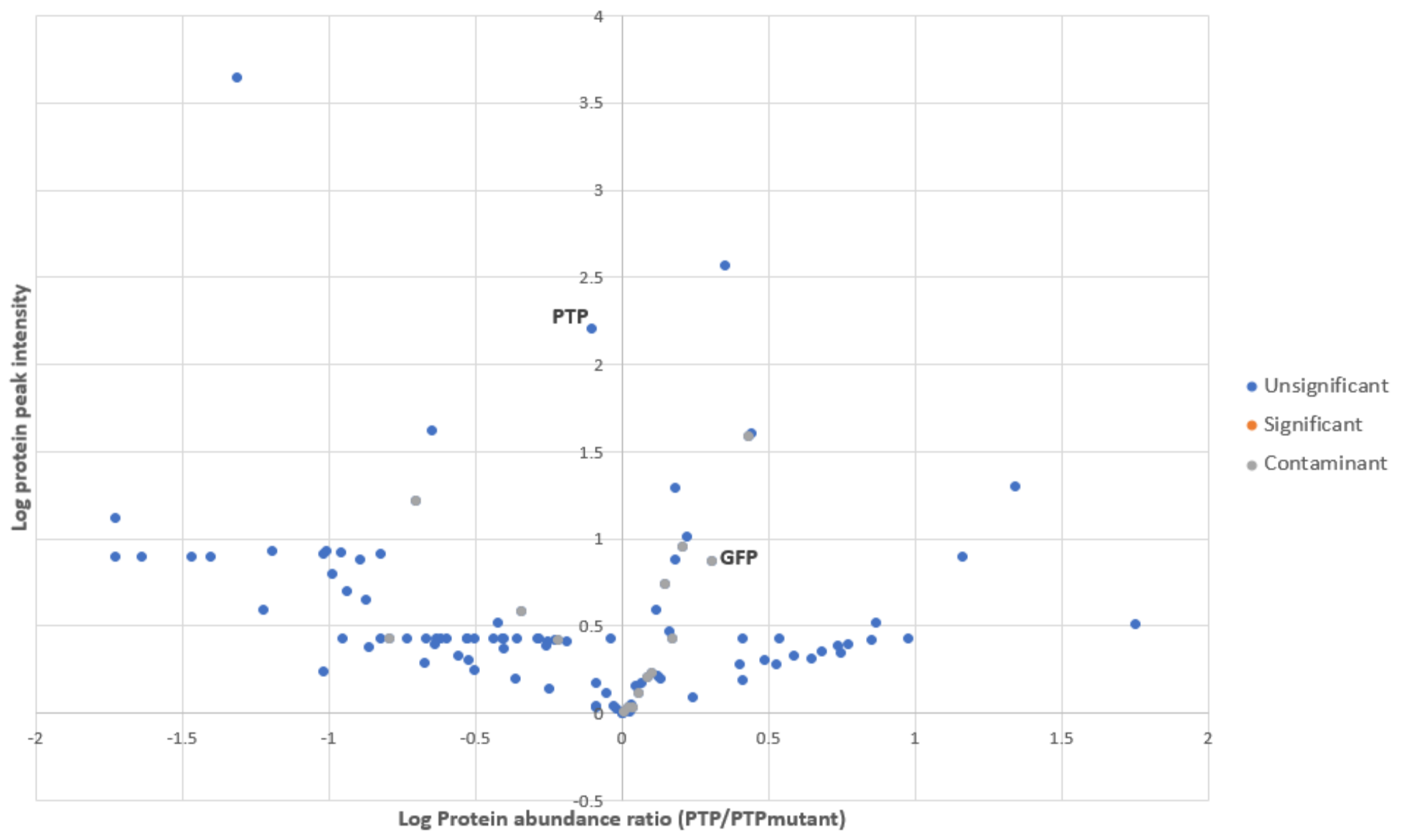

Supplementary Figure S1. Mass spectrometric proteomic analysis of purified PTP-GFP proteins vs PTPM-GFP proteins. PTP-GFP and PTPM-GFP proteins were prepared from AcMNPV infected larvae and precipitated by GFP antiserum. The precipitation experiments were conducted three times from the same starting material, precipitated proteins were analyzed with LC-MS/MS analysis using MaxQuant software and the statistical analysis was performed with 
the Perseus module. The Y-axis shows the relative abundance (peak height) as log (10) values, and X-axis shows the log (10) of the abundance ratio of proteins precipitated in the PTP-GFP group over proteins precipitated in the PTPM-GFP group. Proteins that are significantly enriched in the PTP-GFP sample over the PTPM-GFP control are shown in orange $(\mathrm{P}<0.01)$. Proteins that are not significantly enriched are shown in blue. Contaminants such as keratins and trypsin are shown in grey.

\section{Supplementary Table S1: Primers used in this study}

\begin{tabular}{|c|c|c|c|}
\hline $\begin{array}{l}\text { Primer } \\
\text { number }\end{array}$ & $\begin{array}{l}\text { Primer } \\
\text { name }\end{array}$ & Sequence (5' to 3') & Function \\
\hline 1 & $\begin{array}{l}\text { GFP-PTP } \\
\text { Fw } 1\end{array}$ & CCCCCGCTAGCATGGTGAGCAAGGGCGAGGAG & Amplify GFP ORF for GFP-PTP \\
\hline 2 & $\begin{array}{l}\text { GFP-PTP } \\
\text { Rv } 1\end{array}$ & $\begin{array}{l}\text { TGCCAACGCGCGGGAAACATCTTGTACAGCTCGTCCATG } \\
\text { C }\end{array}$ & $\begin{array}{l}\text { Amplify GFP ORF for GFP-PTP, create overhang } \\
\text { with PTP ORF }\end{array}$ \\
\hline 3 & $\begin{array}{l}\text { GFP-PTP } \\
\text { Fw } 2\end{array}$ & $\begin{array}{l}\text { GCATGGACGAGCTGTACAAGATGTTTCCCGCGCGTTGGC } \\
\text { A }\end{array}$ & $\begin{array}{l}\text { Amplify PTP ORF for GFP-PTP, create overhang } \\
\text { with GFP ORF }\end{array}$ \\
\hline 4 & $\begin{array}{l}\text { GFP-PTP } \\
\text { Rv } 2\end{array}$ & GGGGATGCATTTAAATTAATAAATCTTGAACGTA & Amplify PTP ORF for GFP-PTP \\
\hline 5 & $\begin{array}{l}\text { PTP-GFP } \\
\text { Fw } 1\end{array}$ & CCCCCGCTAGCATGTTTCCCGCGCGTTGGCA & Amplify PTP ORF for PTP-GFP \\
\hline 6 & $\begin{array}{l}\text { PTP-GFP } \\
\text { Rv } 1\end{array}$ & $\begin{array}{l}\text { CTCCTCGCCCTTGCTCACCATAATTAATAAATCTTGAACG } \\
\text { TA }\end{array}$ & $\begin{array}{l}\text { Amplify PTP ORF for GFP-PTP, create overhang } \\
\text { with GFP ORF }\end{array}$ \\
\hline 7 & $\begin{array}{l}\text { PTP-GFP } \\
\text { Fw } 2\end{array}$ & $\begin{array}{l}\text { TACGTTCAAGATTTATTAATTATGGTGAGCAAGGGCGAG } \\
\text { GAG }\end{array}$ & $\begin{array}{l}\text { Amplify GFP ORF for GFP-PTP, create overhang } \\
\text { with PTP ORF }\end{array}$ \\
\hline 8 & $\begin{array}{l}\text { PTP-GFP } \\
\text { Rv } 2\end{array}$ & GGGGGATGCATTTACTTGTACAGCTCGTCCATGC & Amplify GFP ORF for PTP-GFP \\
\hline
\end{tabular}




\section{Chapter}

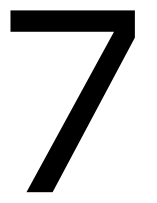

Baculovirus invasion of the lepidopteran central nervous system

Han Y, Groothuis J, Suijkerbuijk H, Wang Y, van Lent JMW, Smid HM, Ros VID. Manuscript in preparation. 


\begin{abstract}
Baculoviruses induce hyperactivity and pre-death climbing (tree-top disease) in their host, processes which are thought to enhance virus transmission. Though some baculovirus genes have been studied for their role in inducing behavioural changes, host pathways involved are largely unknown. Insect behaviour is primarily regulated via the host central nervous system (CNS), therefore, baculoviruses possibly affect host behaviour via invading or affecting the host CNS. Since serotonin has been reported to play a role in insect locomotion, we studied the distribution of serotonergic neurons in the larval brain in more details. We first made a 3D model for the third instar Spodoptera exigua larval brain, which shows the shape of different neuropils and their relative position in the brain. This was done by immunolabelling of serotonin in the larval brain. Next, we described the distribution of serotonergic neurons in the larval brain, including the location and the number of serotonergic neurons in each serotonergic cell cluster. As a proof of concept, we also investigated whether AcMNPV infects the S. exigua larval brain by using a recombinant virus expressing green fluorescent protein. Confocal laser scanning microscopy and electron microscopy results confirmed that AcMNPV is present and replicates in the S. exigua brain. Moreover, it seems that AcMNPV only invades specific regions of the brain, following a temporal pattern. The results provide further information on the mechanism of baculovirus-induced behavioural manipulation.
\end{abstract}




\section{Introduction}

Baculoviruses are arthropod-infecting viruses characterized by rod-shaped virions. Baculoviruses contains double-stranded, circular supercoiled DNA genomes (Rohrmann, 2013; Slack and Arif, 2007). Two types of virions are produced during an infection cycle: budded virions (BVs) and occlusion derived virions (ODVs). BVs are responsible for triggering systemic infection within the host while ODVs are responsible for initiating infection in the host's epithelial midgut cells and for the horizontal transmission of baculoviruses (van Oers et al., 2015; Williams et al., 2017). Baculoviruses are known to induce behavioural changes in their caterpillar hosts, including hyperactivity and pre-death climbing behaviour (tree-top disease) (Hoover et al., 2011; Kamita et al., 2005; van Houte et al., 2012). These changes are thought to spread the viruses over a larger surface of plant foliage, thus enhancing virus transmission (Hoover et al., 2011; Kamita et al., 2005; van Houte et al., 2012). Previous studies found that the protein tyrosine phosphatase (ptp) gene from the baculoviruses Autographa californica multiple nucleopolyhedrovirus (AcMNPV) and Bombyx mori (Bm)NPV were involved in inducing hyperactivity in Spodoptera exigua and B. mori larvae, respectively. Therefore, $p t p$-induced hyperactivity might be an evolutionarily conserved strategy of a subclade of baculoviruses (Group I NPVs) to enhance virus transmission (Kamita et al., 2005; Katsuma et al., 2012; van Houte et al., 2012).

Parasites may achieve host manipulation by invading or affecting the central nervous system (CNS) of the host (Adamo, 2002; Libersat et al., 2009; Ohkawara and Aonuma, 2016; PerrotMinnot and Cezilly, 2013). For example, the emerald jewel wasp, Ampulex compressa, injects venom precisely into the brain of the cockroach Periplaneta americana and as a result reduces the spontaneous movements of the cockroach, making it a better food supply for the wasp's offspring (Rosenberg et al., 2007). A major target of the venom appeared to be the octopaminergic network in the brain of the cockroach (Rosenberg et al., 2007). A role for octopamine was also observed for the caterpillar Manduca sexta parasitized by the parasitoid wasp Cotesia congregata. The parasitized caterpillar functions as a bodyguard for the wasp's offspring after the wasp larvae have egressed from the caterpillar and form cocoons. In addition, the caterpillar shows declined feeding and locomotion behaviour. These behavioural alterations are accompanied by a sharp increase in octopamine content in the larval CNS (Adamo, 2002). Likewise, baculoviruses may induce behavioural changes in their host by invading the host's CNS. This hypothesis is supported by the evidence that baculoviruses have been detected in the cerebral, thoracic and abdominal ganglia cells of infected hosts (Herz et al., 2003; Torquato et 
al., 2006) and expression of baculoviral genes has been detected in host neural glial cells (Knebel-Morsdorf et al., 1996) and brains (Katsuma et al., 2012; Wang et al., 2015).

Serotonin, dopamine and octopamine are examples of biogenic amines. Biogenic amines are called neurotransmitters in vertebrates. However, they have broader functions in invertebrates, serving as neurotransmitters, neuromodulators and/or neurohormones (reviewed by Fieber, 2017). Biogenic amines control a wide range of physiological processes in invertebrates and are also involved in host behavioural manipulation (Adamo, 2002; Rosenberg et al., 2007; Tain et al., 2006). For example, third instar Drosophila melanogaster larvae present light-controlled locomotion behaviour. Reducing serotonin signalling within serotonergic neurons intensified this behaviour, suggesting that serotonin plays an important role in controlling this behaviour (Rodriguez Moncalvo and Campos, 2009). The gammarid Gammarus lacustris infected by polymorphid cystacanths shows increased movement towards light compared with nonparalyzed individuals. Injected with serotonin into G. lacustris mimicked this behaviour, suggesting serotonin also plays an important role in this process (Maynard et al., 1996).

The CNS of lepidopteran larvae consists of a brain and a ventral nerve cord. The brain, or cerebral ganglion (CRG), has two hemispheres and the ventral nerve cord consists of the subesophageal ganglion (SEG) or gnathal ganglion (GNG), three thoracic ganglia and seven abdominal ganglia (Cantera et al., 1995; Huetteroth et al., 2010; Ito et al., 2014). The morphology of brain neuropils and the layout of aminergic neurons are different in different larval stages (Huetteroth et al., 2010). Although studies have been conducted on the lepidopteran CNS (Audsley et al., 2000; Huetteroth et al., 2010), their focus on the pupal or adult brain make them an unsuitable reference for a study on manipulation of larval brains. Therefore, in this study, we first made a 3D model for the brain of third instar S. exigua larvae, showing the general brain morphology and the relative position of the different neuropils. Next, we investigated serotonin in the larval brain in more details. We described the distribution of serotonergic neurons in the larval brain, including the location and the number of serotonergic neurons in each serotonin cell cluster. As a proof of concept, we also investigated whether AcMNPV infects the $S$. exigua larval brain. Our results showed that AcMNPV infects the brain starting from 2 days post infection. Furthermore, AcMNPV forms OBs in the larval brain, a measure for viral replication in the host brain. Thus, we hypothesize that AcMNPV may achieve induction of hyperactivity in S. exigua larvae by invading the host CNS. 


\section{Materials and Methods}

\section{Insect and virus}

Spodoptera exigua larvae were reared on artificial diet as described before (Han et al., 2015). A recombinant baculovirus AcMNPV able to make OBs and with green fluorescent protein (GFP) fused to the nucleocapsid protein VP39 and functional OBs were able to form(Mu et al., 2014) was used to visualize virus infection in the larval brain and GNG.

\section{Immunolabeling of brains for localisation of serotonergic neurons and neuropil reconstruction}

Newly moulted third instar larvae were used for labelling of brains and GNGs using a commercially available antiserum raised against serotonin (Millipore Cat\# AB938). In addition, propidium iodide was used to visualize the nuclei. Cold-sedated larvae were decapitated in icecold PBS. The cuticle was removed and brains were dissected using a pair of fine sharpened tweezers (Dumont \#5, Sigma). Dissected brains and GNGs were flat aligned on a microscope glass and fixed in that orientation by adding a droplet of freshly prepared ice-cold $4 \%$ formaldehyde for $10 \mathrm{~min}$. This way the brain was fixed in line with the neural axis of the ventral nerve cord, including the GNG. After 10 min the preparation remained stable in this aligned position when transferred to the same fixative solution at room temperature (RT) and fixed overnight. Further procedures for immunolabelling and antiserum specificity controls were as described by van der Woude and Smid (2017). Finally, dehydrated brains and GNGs were mounted in DPX (Sigma).

\section{Image acquisition and processing}

Preparations were scanned with a Zeiss LSM510 microscope equipped with a $25 \times$ or $40 \times$ oil immersion objective (Plan-Neofluar $25 \times / 0.8$ ). AlexaFluor ${ }^{\circledR} 488$ staining was imaged using the 488-nm line of an Argon laser with a 505- to 530-nm BP filter; the propidium iodide nuclear counterstain was imaged using the same excitation wavelength but with a 585-nm LP filter. Preparations were scanned at $1,024 \times 1,024$ or $2,048 \times 2,048$ pixels with a digital magnification of 0.8 and a step size of $2 \mu \mathrm{m}$. Since the refractive index of the mounting medium matched that of the immersion oil, we did not use a Z-distance correction.

The 3D model of brain neuropils was constructed using the Amira 5.4.2 (Visage Imaging) Segmentation Editor. Background staining of 5HT-like-immunofluorescence (5HT-L-IR) and propidium iodide was sufficient to recognize and assign unique materials to voxels several neuropils. Preparations were assigned every 1-5 slices and completed with the Interpolation 
option of Amira. The SurfaceGen and Animation modules were used to create 3D images based on the segmentation.

The number of cell bodies was only analysed in brains in which 5HT-L-IR clusters were clearly visible and the best-stained hemisphere was selected for analysis of individual clusters. Due to tissue damage, not all identified clusters could be counted in every sample. In Amira, each cluster was assigned to a separate LabelField and each cell was segmented with an individual Material. During this process, the propidium iodide counterstain was used for orientation, but not for other purposes. After segmentation, results were exported with the MaterialStatistics module and collected in an MS Excel spreadsheet for further analysis. Although volumetric information on the labelled 5HT-L cell bodies was created by this procedure, the extensive flattening of the preparations during mounting is likely to confound these data; therefore, these were not used for further analysis.

\section{Infection of larvae and virus detection in host CNS}

Early third instar S. exigua larvae were infected with AcMNPV-GFP as described in Han et al. (2015). Larvae were infected with a viral dose of $10^{8} \mathrm{OBs} / \mathrm{ml}$ using droplet feeding. Mockinfected larvae were used as uninfected controls and were fed with a virus-free sucrose solution as described before (Han et al., 2015). After infection, larvae were placed individually in 12well plates, provided with a piece of artificial diet, and reared at $27^{\circ} \mathrm{C}$.

At one, two and three days post infection (dpi), the brain and GNG of infected and mockinfected larvae were dissected as described above and checked for virus infection by confocal laser scanning microscopy (CLSM). For CLSM, a similar procedure was used as for immunolabelling but only propidium iodide staining was included and the samples were not dehydrated but mounted in Vectashield (VECTOR Laboratories) and sealed with transparent nail polish. Preparations were scanned with a Zeiss LSM510 microscope equipped with a 40× oil immersion objective (Plan-Neofluar 40×/1.3). Image acquisition was performed as described above.

To investigate AcMNPV infection in the $S$. exigua larvae CNS at higher resolution, brains and GNGs of AcMNPV-GFP-infected larvae were checked by transmission electron microscopy (TEM). Briefly, the brains and GNGs of virus-infected and mock-infected larvae were dissected at 3 dpi and submersed in the fixative solution (2\% glutaraldehyde, $4 \%$ paraformaldehyde, $1 \mathrm{mM} \mathrm{CaCl} 2$ in $50 \mathrm{mM}$ PIPES). The fixation was performed at $4{ }^{\circ} \mathrm{C}$ overnight. After rinsing three times in the washing buffer $\left(0.3 \mathrm{M}\right.$ PIPES, $\left.1 \mathrm{M} \mathrm{CaCl}_{2}\right)$, the samples were dehydrated by 
$50 \%, 70 \%$ and $90 \%$ ethanol each for $15 \mathrm{~min}$. Subsequently, the samples were infiltrated by LR White (London Resin Company) with different ratios of ethanol (LR White/90\% ethanol 1:1, 2:1, 3:1 for $45 \mathrm{~min}$ each; pure LR White, overnight at $4{ }^{\circ} \mathrm{C}$ ). Lastly, the brains and GNGs were placed in a gelatine capsule and filled with pure LR White embedding medium. The resin was polymerized at $50{ }^{\circ} \mathrm{C}$ for $24 \mathrm{~h}$ and the end products were stored at RT. Thin slices with a thickness of $70 \mathrm{~nm}$ were obtained by sectioning the capsule of the plastic-embedded preparation. The sections were observed under a JEOL JEM1011 TEM after staining with uranyl acetate and lead citrate.

\section{Results}

\section{Orientation of the larval CNS and nomenclature}

The S. exigua larval brain is located dorsal to the esophagus, while the GNG is located ventral to the esophagus. A previous study used the body axis as the reference to describe the position of the brain and the GNG (Huser et al., 2012). Since the brain is positioned at an angle of almost $180^{\circ}$ to the GNG in lepidopteran larvae (Figure 1A), when the CNS is described as a whole, it is necessary to switch between axes at the border of the brain and GNG (Huser et al., 2012). Ito et al. (2014) proposed to use the neuroaxis to describe the insect nervous system. The neuroaxis (Figure 1B) describes the relative position along the axis of the ventral nerve cord, as obtained for the brain when it is flipped forward over $180^{\circ}$ and thereby aligned with the GNG and ventral nerve cord (VNC), such as we achieved by our alignment at the beginning of the tissue fixation. Therefore, when describing the CNS as a whole, it is not needed to switch between axes anymore. As a result, the dorsal side of the brain along the body axis is the ventral side along the neuraxis. Most importantly, the neuroaxis complies with the single systematic nomenclature for the insect adult brain proposed by Ito et al. (2014). In our study, the naming of neuropils was adopted to this nomenclature. When naming was impossible due to the differences between the adult and larval brain, names were given based on a reconstruction of neuropils in fifth instar M. sexta larvae (Huetteroth et al., 2010). 

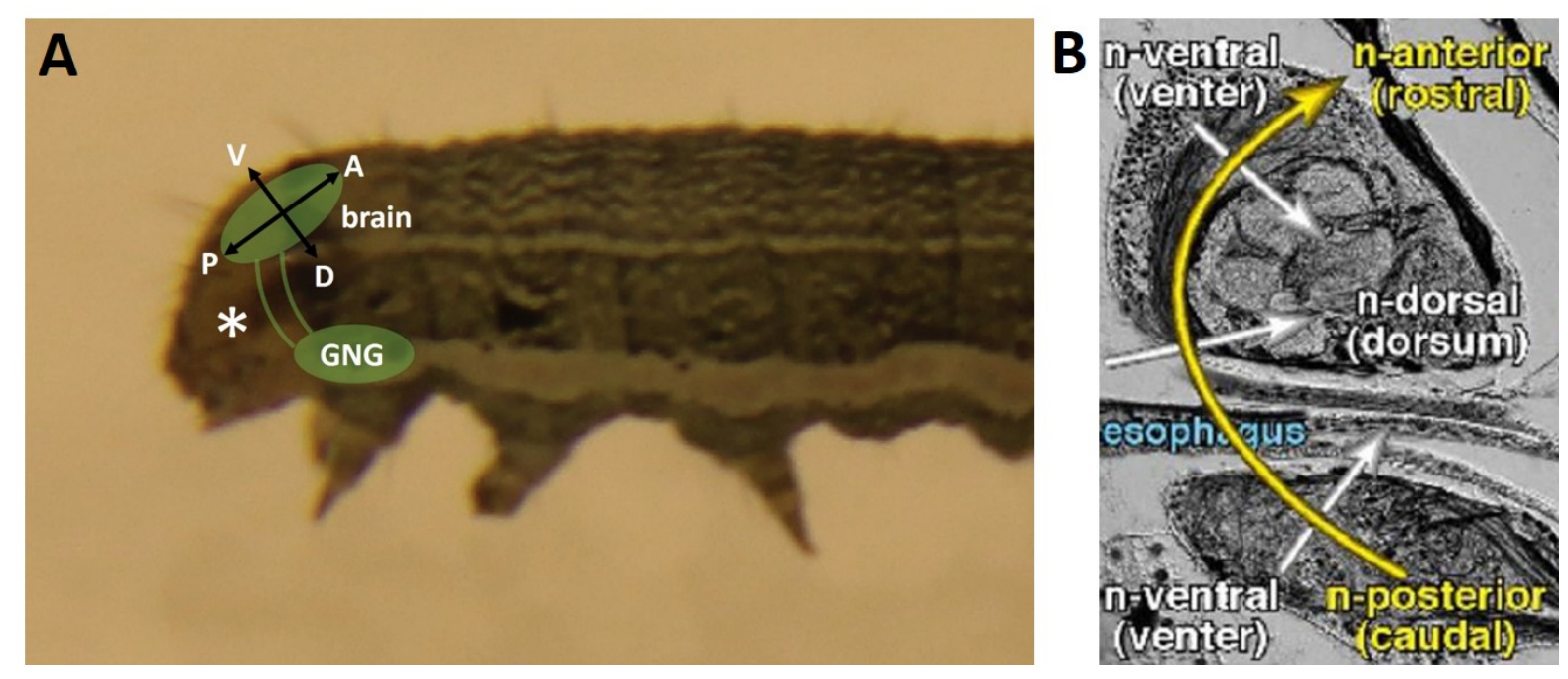

Figure 1. Orientation of the Spodoptera exigua larval brain A) Orientation of axes. V = ventral; D = dorsal; $\mathrm{P}=$ posterior; $\mathrm{A}=$ anterior; $\mathrm{GNG}=$ gnathal ganglia. The * indicates the position of the esophagus. B) The neuraxis as proposed by Ito et al. (2014) is shown as a yellow arrow. The area left of the yellow arrow is ventral, the area right of the yellow arrow is dorsal. The n- prefix is used to indicate that the direction are along the neuraxis instead of the body axis (taken from Ito et al. (2014).

\section{$3 D$ reconstruction of neuropils in the brain}

To study S. exigua brain in more details, we labelled the serotonin in the larval brain. Neuropils are defined as a synaptically dense region that composed of mostly unmyelinated axons, dendrites and glial cell processes and with a relatively low number of cell bodies. Neuropils were visible in the serotonin labelling preparations, therefore, a 3D reconstruction of neuropils in the larval brain was made based on one representative serotonin preparation. Neuropils of five supercategories could be identified in the third instar S. exigua larval brain: the larval optical centre (LOC); the central complex (CC); the superior neuropils (SNP); the larval antennal centre (LAC) and the mushroom body (MB). The MB is further subdivided into four neuropils: the calyx (CA); the pedunculus (PED); the medial lobe (ML); and the vertical lobe (VL). The whole brain is covered by the cell body rind. More details on the position and morphology of S. exigua neuropils are shown in Figure 2. 
A


Figure 2. Model of the Spodoptera exigua larval brain at the third instar. A) Ventral view, B) Dorsal view. Orientation is as indicated in the panel, $\mathrm{A}=$ anterior; $\mathrm{L}=$ lateral $(\mathrm{L})$. Transparent grey colour indicates the outline of the brain and contain the cell body rind. Light blue indicates the area of superior neuropils $(\mathrm{SNP}) . \mathrm{CA}=$ calyx; $\mathrm{LOC}=$ larval optical centre; $\mathrm{VL}=$ ventral lobe; $\mathrm{ML}=$ medial lobe; $\mathrm{PED}$ $=$ pedunculus; $\mathrm{CB}=$ central body; $\mathrm{LAC}=$ larval antennal centre. Scale bars represent $50 \mu \mathrm{m}$. 


\section{Serotonergic cell bodies in the brain}

Previous studies on the serotonergic nervous system of larval stages of insects mainly focused on Drosophila melanogaster (Huser et al., 2012; Schoofs et al., 2014). However, in our study, the locations of $S$. exigua 5HT-L-IR cells were found to differ too much from those in $D$. melanogaster to use the same nomenclature. Therefore, a nomenclature based on the location of the 5HT-L-IR cell bodies was used. On average, 18 5HT-L-IR cell bodies were identified per hemisphere in the third instar $S$. exigua larval brain. These cells were divided into eight clusters, each present in both hemispheres. The naming of each cluster was based on its location in the brain. For the nomenclature of cell clusters, the first letter represents dorsal (D) or ventral (V). When the cluster was positioned in the middle of the brain (between the dorsal and ventral side), the corresponding letter is left out. The second letter represents anterior (A) or posterior (P) and the last letter represents medial (M) or lateral (L). An overview of all 5HT-L-IR clusters is provided in Table 1. Identification of serotonergic cell clusters was based on four factors: the location of the cells in the brain, the relative position of cells with the cluster, the brightness of the immunolabelling and the shape and/or size of the cells.

The number of 5HT-L-IR neurons in each cell cluster was also counted. The range of the number of cells in each cluster (over the different preparations), the average number of cells per cluster and the number of cells that was most often counted per cluster (mode) are shown in Table 1. Only cells that were identified at least in two preparations were used. Ventral cell clusters are shown on a ventral map of the brain (Figure 3). Dorsal cell clusters are shown on the dorsal map of the brain (Figure 4). Medial cell clusters are shown on both maps.

The ventral anterior medial (VAM) cluster was on the ventral side of the brain, in a medial anterior position (Figure 3A). On average, the cluster contained 2.06 cells $( \pm 0.24$ (standard deviation, $\mathrm{SD}), \mathrm{n}=17$ ). The cluster mostly consisted of two cells, which were in the same plane. The two cells in this cluster were named VAM medial and VAM lateral cell.

The ventral lateral (VL) cluster was located on the ventral lateral side of the brain (Figure 3B). The VL cluster contained two cells in all 13 preparations in which they were identified. The two cells were located in an anterior and posterior direction. The two cells were named VL anterior and VL posterior cell.

The ventral posterior medial (VPM) cluster was positioned medial to the LAC and flanking the esophagus (Figure $3 C$ ). On average, the cluster consisted of 1.50 cells $( \pm 0.53, n=10)$. The 
cluster most often contained two cells, one VPM medial and one VPM lateral cell. The two cells were most often positioned at a medial/lateral position.

The anterior lateral (AL) cell body was located in the anterior protocerebrum, lateral and dorsal to the VAM cluster (Figure 3D). The cluster contained only one cell in all counted preparations $(n=14)$. Some weakly immunoreactive cells were observed in the AL region, however, they were not consistently observed in all preparations We considered this to be due to autofluorescence or aspecific antibody binding and left such cells out from the analysis.

The dorsal lateral (DL) cluster was flanking the lateral protocerebrum halfway along the anterior-posterior axis. In all counted samples, the cluster contained two cells $(n=14$; Figure 4A). These two cells were directly next to each other and labelled as DL medial and DL lateral cell.

The dorsal anterior lateral (DAL) cluster was located anterior to the DL cluster, and ventrallateral to the LOC (Figure 4B). It contains on average $1.13( \pm 0.34, \mathrm{n}=16)$ cells, where mostly only one cell was observed.

The dorsal anterior medial (DAM) cluster was located along the anterior midline, dorsal to the VAM cluster (Figure 4C). On average, the cluster contained 6.21 cells $( \pm 0.58, n=14)$. The cluster most often consisted of 6 cells, which were not in the same plane. The six cells were labelled as DAM dorsal anterior (DA), DAM dorsal posterior (DP), DAM medial anterior (MA), DAM medial posterior (MP), DAM ventral anterior (VA) and DAM dorsal lateral (DL) cell.

The posterior lateral (PL) cluster was located anterior to the LAC (Figure 4D). On average, the cluster contained 1.87 cells $( \pm 0.35, n=15)$. Mostly, two cells were identified in this cluster positioned horizontally to each other. The two cells were named PL medial and PL lateral cell 

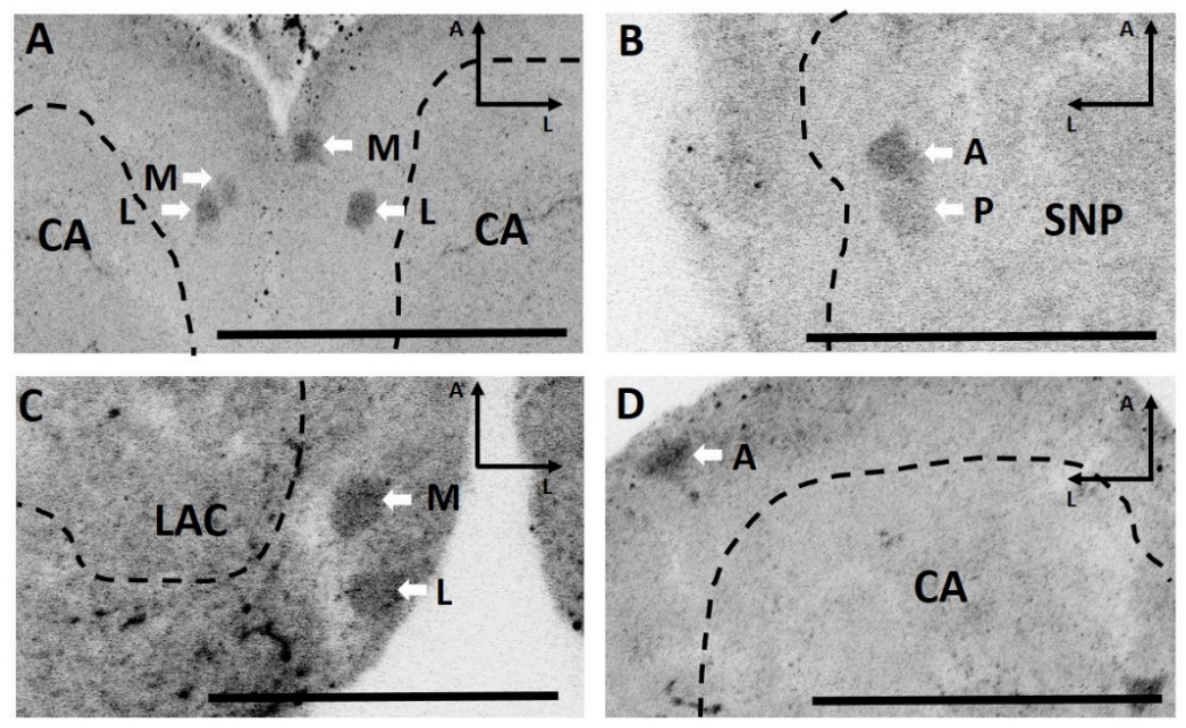

$\mathbf{E}$

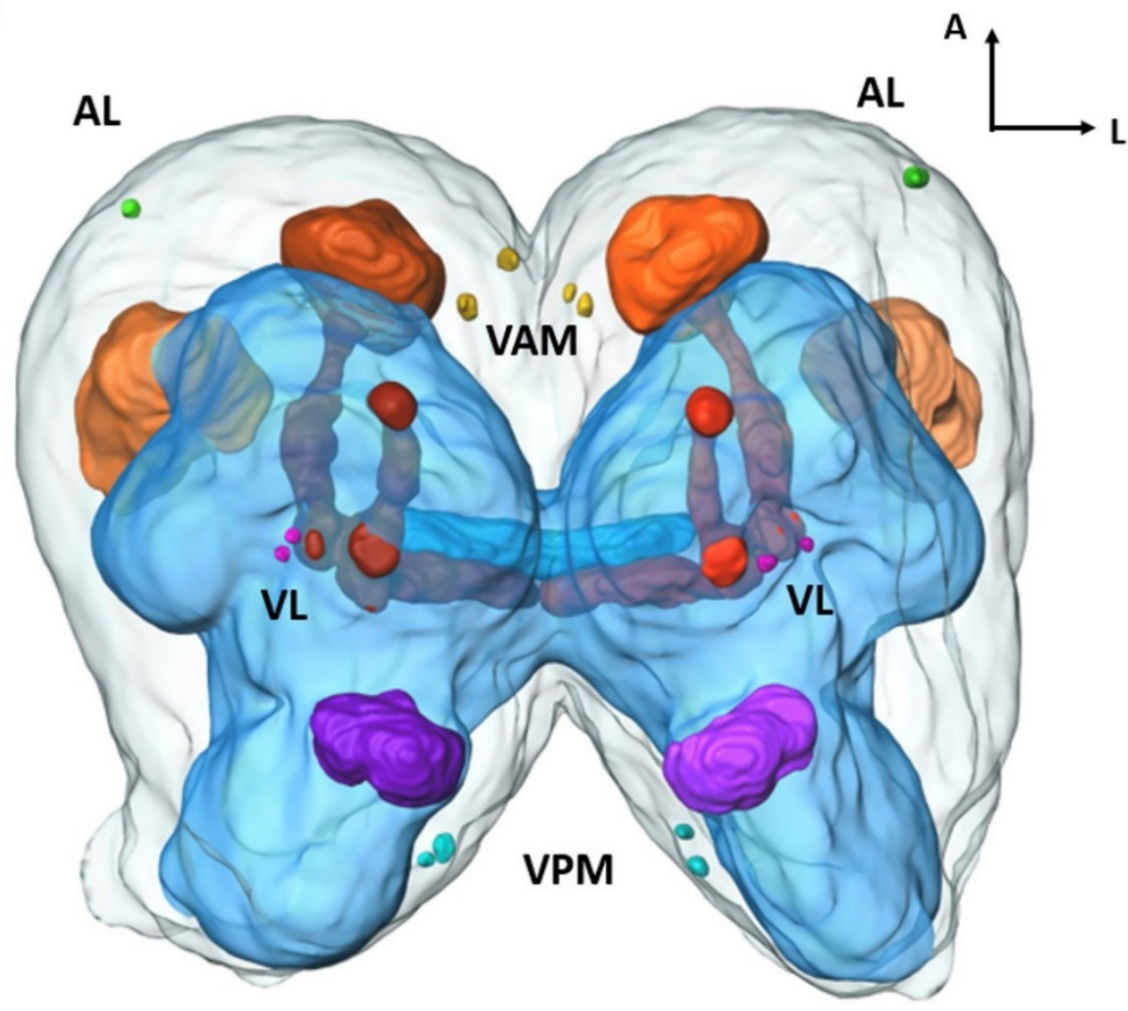

Figure 3. Serotonergic cell clusters in the $S$. exigua third instar brain. Ventral cell clusters and the AL cell are shown. A-D are projections from confocal images of the serotonergic cell clusters in the larval brain. A: VAM cluster (light yellow); B: VL cluster (purple); C: VPM cluster (light blue); D: AL cell (green). E shows the positions of the ventral cell clusters and the AL cell in the third instar S. exigua larval brain. Orientation is as indicated in the panel, $\mathrm{A}=$ anterior; $\mathrm{L}=$ lateral $(\mathrm{L}) \mathrm{VAM}=$ ventral anterior medial cluster; $\mathrm{VL}=$ ventral lateral cluster; $\mathrm{VPM}=$ ventral posterior medial cluster; $\mathrm{AL}=$ anterior lateral; $\mathrm{CA}=$ calyx; SNP = superior neuropils; LAC $=$ larval antennal centre. Scale bars represent $50 \mu \mathrm{m}$. 

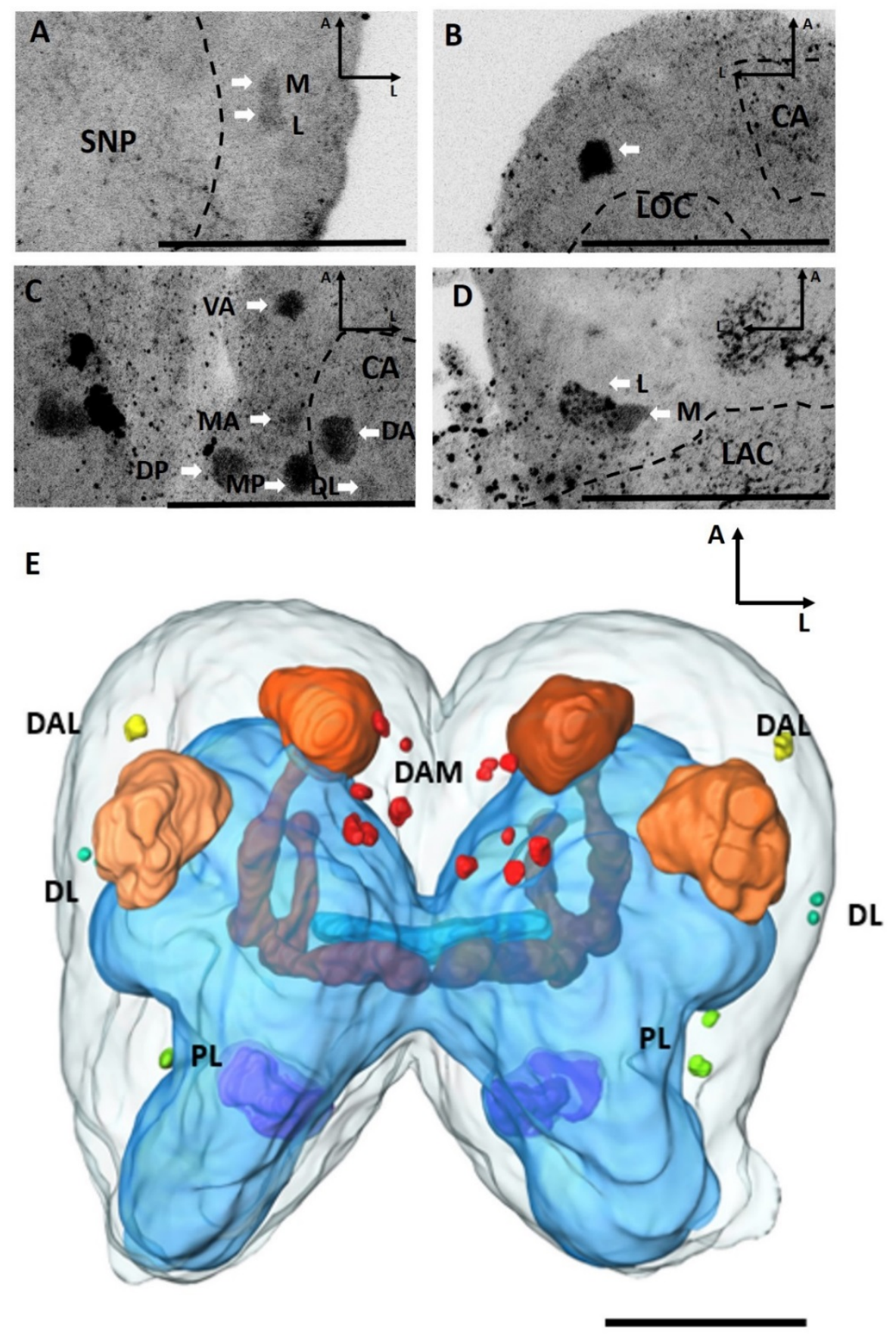

Figure 4: Dorsal cell clusters and the PL cell cluster. A-D are projections from confocal images of the serotonergic cell clusters in the larval brain. A: the DL cluster (dark green); B: the DAL cluster (yellow); C: the DAM cluster (red); D: the PL cluster (dark green). E shows the positions of dorsal cell clusters and PL cell cluster in S. exigua larval brain. Orientation is as indicated in the panel, A = anterior; $\mathrm{L}=$ lateral $(\mathrm{L}) . \mathrm{DL}=$ dorsal lateral cluster; $\mathrm{DAL}=$ dorsal anterior lateral cluster; $\mathrm{VPM}=$ ventral posterior medial cluster; $\mathrm{PL}=$ posterior lateral; $\mathrm{CA}=$ calyx; $\mathrm{SNP}=$ superior neuropils; $\mathrm{LAC}=$ larval antennal centre; $\mathrm{A}=$ anterior; $\mathrm{L}=$ lateral. Scale bars represent $50 \mu \mathrm{m}$. 
Although the abovementioned cell clusters were identified in the larval brain, the characteristics used for their identification might need adaptation in future studies. The DAM, DAL and PL clusters were identified with the highest certainty, since they were identified in most of the preparations and since the identified cells showed the strongest signals during immunolabelling. Furthermore, the unique position of the 5HT-L-IR cells within DAM cluster, the strong labelling of DAL cells and the star-shape of the PL lateral cell make them easier to be identified. The AL, DL and VAM clusters were identified with less certainty. This is because cells and the strength of the signal after immunolabelling in these clusters was very similar to the surrounding background. The VL and VPM clusters were identified with the lowest certainty. Both clusters were located at the border of the brain close to the esophagus, which makes them to be damaged easily during dissection. Consequently, both clusters were not identified in all preparations. Moreover, the VPM cluster was located close to an area with a very strong serotonin innervation, which makes this cluster more difficult to be identified due to overexposure of the background. However, in the preparations where the VL and VPM clusters were identified, they were clearly visible.

Table 1: Overview of cell clusters identified per hemisphere in the third instar S. exigua larval brain. The abbreviation and the full name of each cell cluster is listed. The average number of cells per cluster (Mean \pm standard deviation), the number of cells that was most often counted per cluster (Mode), the range of the number of cells per cluster (Range) and the number of preparations counted are listed as well.

\begin{tabular}{|c|c|c|c|c|c|}
\hline $\begin{array}{l}\text { Short } \\
\text { name }\end{array}$ & Full name & Mean \pm SD & Mode & Range & $\begin{array}{l}\text { Preparation } \\
\text { counted }\end{array}$ \\
\hline VAM & $\begin{array}{l}\text { Ventral anterior medial } \\
\text { cluster }\end{array}$ & $2.06 \pm 0.24$ & 2 & 2 to 3 & 17 \\
\hline VL & Ventral lateral cluster & $2.00 \pm 0.00$ & 2 & 2 to 2 & 13 \\
\hline VPM & $\begin{array}{l}\text { Ventral posterior medial } \\
\text { cluster }\end{array}$ & $1.50 \pm 0.53$ & 2 & 1 to 2 & 10 \\
\hline $\mathrm{AL}$ & Anterior lateral & $1.00 \pm 0.00$ & 1 & 1 to 1 & 14 \\
\hline PL & Posterior lateral & $1.87 \pm 0.35$ & 2 & 1 to 2 & 15 \\
\hline DAM & $\begin{array}{l}\text { Dorsal anterior medial } \\
\text { cluster }\end{array}$ & $6.21 \pm 0.58$ & 6 & 5 to 7 & 14 \\
\hline DAL & $\begin{array}{l}\text { Dorsal anterior lateral } \\
\text { cluster }\end{array}$ & $1.13 \pm 0.34$ & 1 & 1 to 2 & 16 \\
\hline DL & Dorsal lateral cluster & $2.00 \pm 0.00$ & 2 & 2 to 2 & 14 \\
\hline
\end{tabular}




\section{Virus detection in the larval brain by CLSM and EM}

To check whether the baculovirus AcMNPV infects the $S$. exigua larval brain and GNG, CLSM was used to visualize GFP-labelled nucleocapsids inside the brain and GNG. At 1 dpi, no GFP signal was first detected in the brains or GNGs from mock- and AcMNPV-infected larvae. The GFP signal started to appear at the lateral part of the brain at 2 dpi. The intensity of the GFP signal differed for independent samples, indicating that the exact course of the infection varies between individuals. At 2 dpi, the GFP signal was mainly detected in the surrounding tissues of the brain and GNG. At 3 dpi, the GFP signal was observed in all brain and GNG preparations and the signal was much brighter than that at 2 dpi. Intriguingly, GFP was not observed all over the brain and GNG, but only in specific cell bodies. However, at this stage it is difficult to pinpoint the exact cell body type and future research is needed to find this out (Figure 5). It is noteworthy that the initiation of the infection in the CNS was always symmetrical and that the infection seemed to be initiated from the lateral sides of the brain and GNG, where tracheae branched inside the brain and GNG tissues.

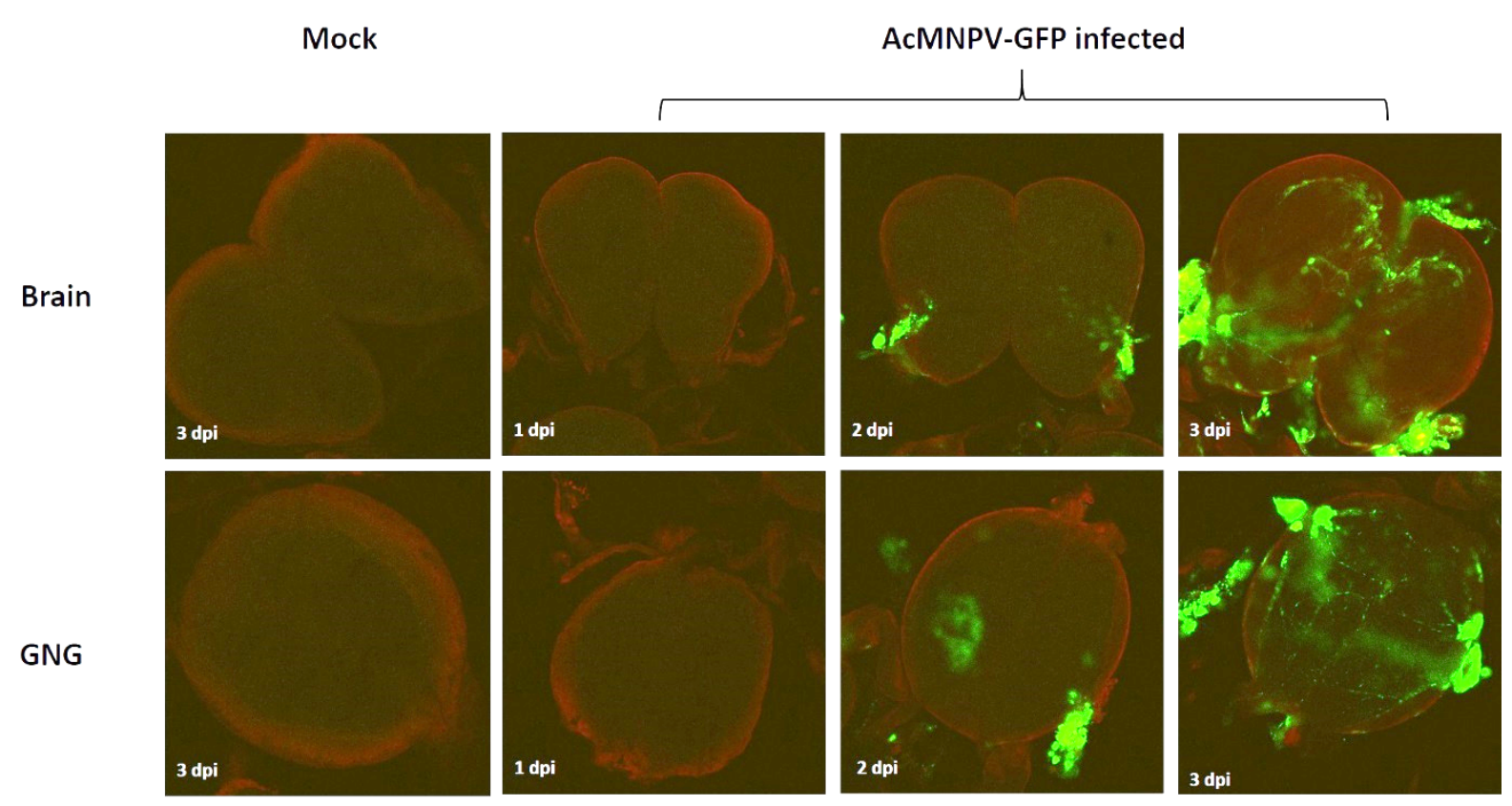

Figure 5. Confocal laser scanning microscopy (CLSM) images of the mock- and AcMNPV-GFPinfected larval brain (upper panel) and GNG (lower panel). The image was the middle CLSM optical section. Only images from mock-infected larvae at 3 dpi are presented. Samples from AcMNPV-GFPinfected larvae were analysed at 1, 2, 3 dpi. Green colour was the GFP signal, Red colour was the propidium iodide stated nucleus. 
Electron microscopy was used to confirm the presence of the virus in the brain and GNG at 3 dpi. As shown in Figure 6, the virus was indeed present in the larval brain. Interestingly, viruses were only observed in a certain type of large cells surrounding neuropils (Figure 6A and 6B, indicated by arrows). Though we did not yet perform co-localization studies on AcMNPV-GFP and biogenic amine secreting neurons to investigate in detail where the virus localized, it seems that, based on the 3D model described above, AcMNPV infects cells in the LOC and LAC region specifically. OBs (polyhedrin protein), fibrillar structures (P10 protein) and virogenic stroma were detected in the nucleus of larval brain cells, indicating that AcMNPV replicates in the brain of infected host (Figure 6C and 6D). Likewise, AcMNPV infects and replicates in the S. exigua larvae GNG (data were not shown).
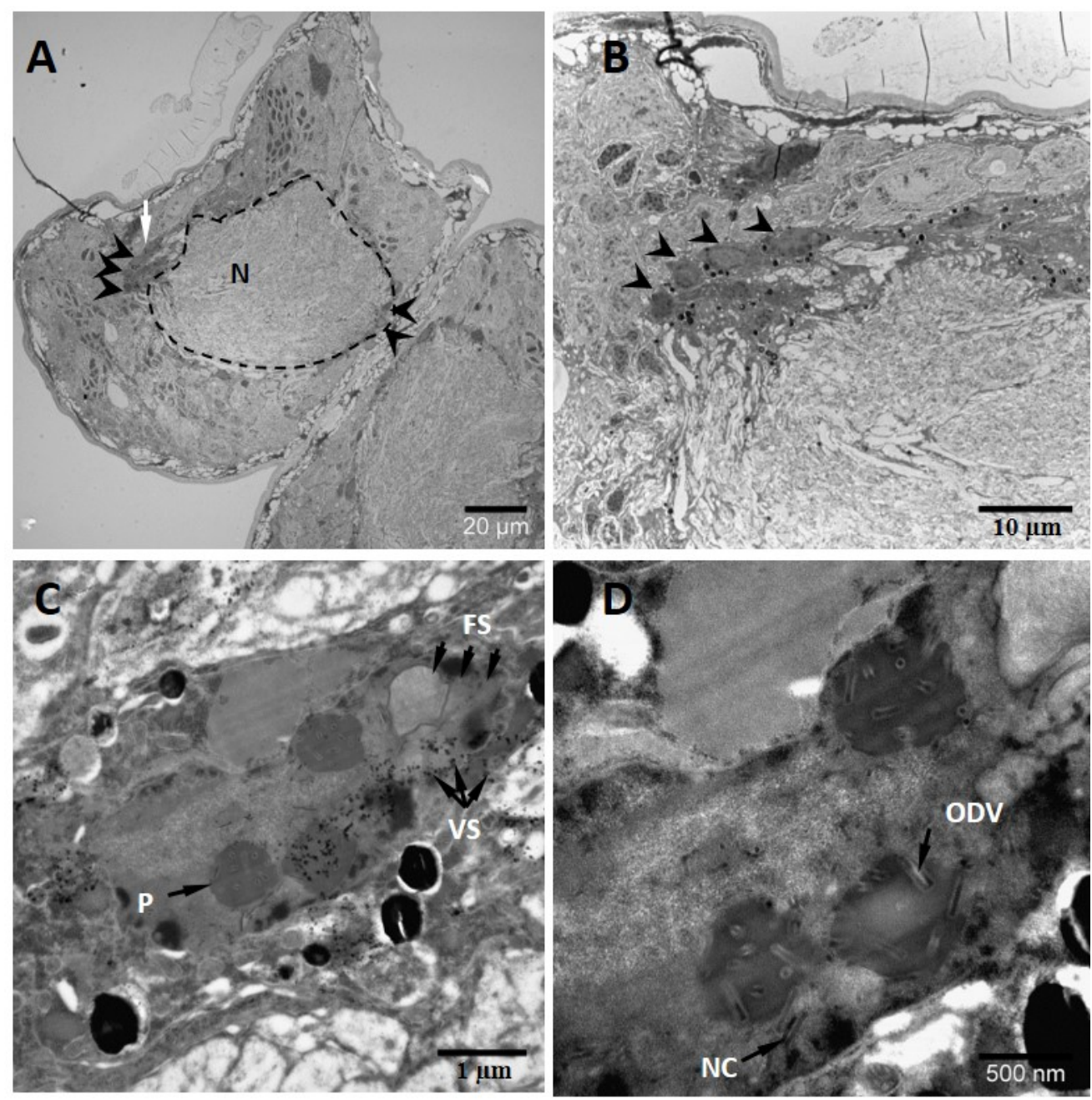

Figure 6. Representative electron microscopy images of the brain from an AcMNPV-infected larva at 3 dpi. A. Overview of a single hemisphere of an infected larval brain. The area within the dashed circle is the neuropil $(\mathrm{N})$. Black arrowheads indicate cells that were infected by viruses (not all cells were scanned for virus infection). B. A close-up of the area indicated by the white arrow in Figure A. Black arrowheads indicate infected cells. C. The nucleus of an infected cell in the brain. OBs (polyhedra, P), 
virogenic stroma (VS) and typical fibrillar structures (FS, formed by P10 protein) were observed. D. Detection of ODVs and nucleocapsids (NC) in the nucleus of an infected cell.

\section{Discussion}

Many parasites, including baculoviruses, manipulate their host's behaviour to enhance their transmission or survival. Since behaviour is primarily regulated via the host's CNS, parasites may affect host behaviour by affecting or invading the host's CNS (Adamo, 2002; Ohkawara and Aonuma, 2016). In order to unravel the underlying mechanisms of AcMNPV-induced hyperactivity in $S$. exigua larvae, this study aimed to provide more insight into the S. exigua larval CNS. Identification of the structure and location of different S. exigua brain areas will enable further research into the role of the brain in AcMNPV-induced hyperactivity in S. exigua. First, we constructed a 3D model of a third instar $S$. exigua larval brain based on immunolabelling of serotonin. Five supercategories of neuropils were identified in the larval brain: LOC, MB, CC, SNP and LAC. In MB, four neuropils were identified, CA, PED, ML and VL. The third instar S. exigua larval brain shares a high similarity with the best and only studied lepidopteran brain so far, the M. sexta larval brain (fifth instar). Four of the five neuropil supercategories (LOC, MB, CC and LAC) and several neuropils (such as CA, PED, ML and VL) identified in the $S$. exigua brain were also identified in the M. sexta larval brain (El Jundi et al., 2009; Huetteroth et al., 2010). There were also differences between these two larval brains, which are likely due to the developmental stage assessed in this study. For example, the protocerebral bridge was only found in the larval M. sexta brain (El Jundi et al., 2009).

Though the 3D model we constructed provides a trustworthy reference for the general shape, location and orientation of the neuropil, it does not represent the in vivo larval brain. The main reason is that the model was only based on a single larval brain. Additionally, in certain steps of the sample preparation (e.g. dehydration) artefacts might have been introduced. Therefore, additional samples need to be investigated to compose a complete model.

Serotonin has been reported to play a role in parasitic manipulation. Therefore, we also investigated the serotonin distribution in the larval brain by immunolabelling. On average, 18 serotonergic cell bodies were identified per hemisphere in the brain. Based on their location in the brain, the cells were divided into eight clusters: the VAM, VL, VPM, AL, PL, DAM, DAL and DL cluster. To our knowledge, no studies have been conducted on the serotonergic neurons in the brain and GNG of lepidopteran larvae. However, similar studies have been conducted in other insect larvae, such as larval fruit flies (D. melanogaster; (Huser et al., 2012; Lundell and 
Hirsh, 1994) and larval honey bees (Apis mellifera; (Boleli et al., 1995). In the larval brain of third instars (the final instar) of D. melanogaster, four cell clusters and 10-11 serotonergic cells were identified per brain hemisphere (Huser et al., 2012). In our study, more cell clusters and serotonergic cells were identified in the brain of S. exigua third instars (this insect has five larval stages). In addition, the relative location of most clusters was different in the $S$. exigua larval brain compared to the D. melanogaster larval brain, which makes it difficult to compare the function of these cell clusters purely based on their location. We did not include the projection and innervation of serotonin neurons in this study due to the variation among the preparations. However, future studies should also focus on this aspect since these will provide more information on the function of serotonin neurons and also on their potential role in baculovirusinduced hyperactivity.

Finally, we investigated whether AcMNPV infects and replicates in the larval CNS. We explored the localization and temporal invasion patterns of AcMNPV in the S. exigua larval brain and GNG. The results showed that AcMNPV not only infected the larval brain and GNG, but also replicated in cells within these tissues. At 2 dpi, viruses started to enter the larval CNS, seemingly via the tracheae. At $3 \mathrm{dpi}$, viruses were detected in all brain and GNG preparations. However, the virus was not detected all over the tissue, but seemed constrained to specific cells. Previously, BmNPV and Helicoverpa armigera (Hear)NPV have been detected in the CNS of infected B. mori (by qPCR) and H. armigera (by fluorescence microscopy) hosts, respectively (Herz et al., 2003; Katsuma et al., 2012; Torquato et al., 2006), however, in these studies, the exact localisation was not investigated. Baculovirus first infect the midgut and from there spreads to other tissues. The brain was infected later than tracheal cells, fat body and malpighian tubes (Passarelli, 2011; Rohrmann, 2013; Soares and Ribeiro, 2005), suggesting that brain was one of the tissues to be infected lastly. Intriguingly, our results obtained by CLSM and EM suggest localization of the virus in specific cells within the host brain, probably in neurons localized within the LOC and LAC areas. However, whether this has a link with virus-induced hyperactivity is unknown at this moment. Wang et al. (2015) compared the brain transcriptomes of BmNPV-infected larvae with that of a healthy control group. The results showed that transcripts involved in circadian rhythms, synaptic transmission and serotonin signalling pathways were significantly upregulated in the virus-infected group, indicating that the host CNS may play an important function in BmNPV-induced hypermobility. However, these changes might be caused by virus infection in general, and whether they play a specific role in inducing hyperactivity still needs further investigation. 
This study is only the first step to explore the role of the host CNS in baculovirus induced hyperactivity. Future studies should focus on how virus infection affects biogenic amines expression and more precisely determine virus localization. Co-localization studies of AcMNPV and biogenic amines (such as serotonin, dopamine and octopamine) in the larval brain and GNG will provide information on whether AcMNPV interacts with biogenic amine secreting cells directly. Future research will also focus on how the virus enters the brain and which role the ptp gene has in this process. Katsuma et al. (2012) showed (using qPCR) that deletion of the $p t p$ gene reduced BmNPV virus titers in the B. mori larval brain. Furthermore, the phosphatase activity of the encoded PTP protein may play a role in virus entry. While Kasuma et al. (2005) showed that the phosphatase activity of BmPTP was not needed for inducing hyperactivity in B. mori larvae, our previous work revealed that the phosphatase activity of AcPTP was needed for inducing hyperactivity in S. exigua larvea (van Houte et al., 2012). Therefore, in AcMNPV infecting S. exigua, the phosphatase activity of AcPTP may be important for virus entry into the larval brain.

Although the abovementioned aspects are certainly worth being investigated, a genome- or proteome-wide approach would give valuable additional information on host gene and/or proteins involved in this process. This could be achieved by comparing host transcriptomes and/or proteomes of WT and $\Delta p t p$ AcMNPV-infected larvae. Comparing transcripts level of key genes that are involved in biogenic amine synthesis between WT and $\Delta p t p$ AcMNPVinfected larvae is of particular interest.

In conclusion, based on immunolabelling of serotonin in the $S$. exigua brain, we constructed a 3D model for the $S$. exigua brain. We also studied the distribution of serotonergic cell bodies in the larval brain: eight cell clusters and 18 serotonergic cell bodies were identified. Finally, we further showed that baculoviruses enter the larval CNS in a spatio-temporal manner and also replicate within the host CNS. The invasion into the CNS started from 2 dpi at the lateral side of the brain and at 3 dpi the virus was detected in the CNS of all investigated larvae. AcMNPV seemed to infect neurons in the LOC and LAC region of $S$. exigua larval brain. The results provide useful information for future studies on the mechanisms of baculovirus-induced behavioural manipulation. 


\section{Acknowledgements}

We thank Els Roode and Hanke Bloksma for their assistance in insect rearing. Iris van der Ham is acknowledged for her help in starting the experiments. Monique van Oers is acknowledged for scientific discussions on the topic and critical reading of the manuscript. 


\section{Chapter}

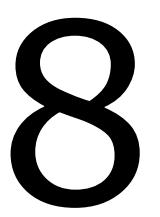

General discussion 


\section{Introduction}

During millions of years of co-evolution, baculoviruses have developed multiple strategies to benefit optimal from their caterpillar hosts (Herniou et al., 2001; Herniou et al., 2003). These strategies include, but are not limited to, behavioural manipulation (induction of hyperactivity and pre-death climbing behaviour) and host immune system modulation. However, how such host modulations are regulated by these viruses is largely unknown. The research described in this thesis aims to answer several key questions on the underlying mechanism of baculovirus induced modulation of host behaviour and the host immune system. As described in the general introduction, this thesis focuses on three main topics: i) the role of the baculovirus egt gene and the timing of light exposure in the induction of pre-death climbing behaviour (tree-top disease); ii) the role of the baculovirus ptp2 gene in modulation of the host immune system; and iii) the mechanism behind ptp-induced hyperactivity, including PTP substrate analysis and virusinvasion of the host central nervous system (CNS).

\section{Mechanisms underlying baculovirus-induced tree-top disease}

The phenomenon that baculovirus-infected caterpillars die at elevated positions (tree-top disease) has first been described more than a century ago (Hofmann, 1891), however, only since the last decade researchers have attempted to unravel the underlying mechanisms. The viral ecdysteroid UDP-glucosyl transferase (egt) gene is the first and only viral gene that has been studied extensively in this process. The egt gene is present in almost all lepidopteran baculoviruses except in one clade of granuloviruses (Ros et al., 2015). The baculovirus Spodoptera exigua multiple nucleopolyhedrovirus (SeMNPV) induces a light-dependent climbing behaviour in third instar S. exigua larvae. An earlier study showed that when infected larvae were not exposed to light after infection, they died at low positions. However, when light was provided from above, infected larvae moved towards the light source and died at elevated positions (van Houte et al., 2014). It had been reported that the EGT enzyme has an effect on how long hosts survive after baculovirus infection (Flipsen et al., 1995; O'Reilly, 1995) and we hypothesized that EGT may exert its effect on tree-top disease by affecting the time to death of the host. In Chapter 3, we showed that the SeMNPV EGT enzyme facilitates pre-death climbing behaviour by extending the survival time of the host (measured as time to death). Larvae infected with a mutant virus lacking a functional egt gene died at lower positions and had a much shorter life span than larvae infected with the wildtype (WT) virus. Mutant virus-infected 
larvae appeared to have died before the normal onset of the climbing behaviour in WT-infected larvae. This observation suggests that death at lower positions of the mutant virus-infected larvae is a side effect of the egt deletion. Moreover, the EGT enzyme inactivates the insect moulting hormone 20E, thereby inhibiting insect moulting (Flipsen et al., 1995; O'Reilly, 1995). Larval moulting is often accompanied by changes in behaviour, and therefore EGT might inhibit moulting related behaviour. When insect moulting and climbing behaviour occur at the same time and just before the insect dies, it may give the impression that EGT induces tree-top disease. Hoover et al. (2011) showed that deletion of the egt gene from Lymantria dispar MNPV (LdMNPV) blocked the induction of tree-top disease in L. dispar larvae (fourth instar) and concluded that the egt gene was required for triggering tree-top disease in L. dispar larvae. However, healthy L. dispar larvae fed in the tree canopy and moved downwards to moult. After infection, the moulting of WT LdMNPV-infected larvae might be inhibited (by EGT), as well as the moulting-related behaviour. Therefore, WT-infected larvae likely stayed at high positions and died there. On the contrary, the moulting of larvae infected with the mutant virus lacking the egt gene was not blocked and these larvae still moved downwards and died at lower positions. When moulting and climbing behaviour occur at different time points, EGT likely does not play a role. Ros et al. (2015) showed that deletion of the egt gene from Autographa californica (Ac)MNPV did not affect the position at which third instars of S. exigua and Trichoplusia ni died. Moulting (and moulting-related climbing) was indeed inhibited in these infected larvae, however, tree-top disease (climbing prior to death) occurred at a much later time point. Therefore, both larvae infected with the WT virus and with the mutant virus lacking the egt gene moved upwards and died at high positions, demonstrating that here egt did not affect tree-top disease. It appears that the effect of the egt gene on tree-top disease depends on many factors, including the developmental stage of the larvae, the host-virus combination, and even the time between the last moult and infection. It can be concluded from the three above mentioned examples that the egt gene does not have a conserved role in baculovirus-induced tree-top disease. Baculoviruses, apparently have evolved different mechanisms to induce treetop disease in different hosts.

Another important factor in baculovirus-induced tree-top disease (at least for SeMNPV) is light. A previous study showed that the location of the light source (above or below the insects) is very important in inducing tree-top disease (van Houte et al., 2014). The results in Chapter 4 showed that the timing of light is also trivial in this process: larvae infected with WT SeMNPV climbed to elevated position between 57 and 67 hours post infection (hpi), however, exposure 
of infected larvae to light (from above) between 43 to $50 \mathrm{hpi}$ was of vital importance to trigger this behaviour. If infected larvae were not exposed to light during this period, or only exposed to light from below, they died at low positions. Whether this phenomenon is conserved among baculoviruses or in a sub-clade of baculoviruses is currently unknown. It seems that light is not needed for LdMNPV-induced tree-top disease in L. dispar: infected larvae still died at high positions even when they were kept in completely dark conditions throughout the experiment (Kelly Hoover, personal communication). Our preliminary data showed that in AcMNPVinfected T. $n i$ larvae, light was not needed to induce tree-top disease, but light was needed in AcMNPV-infected S. exigua larvae to induce pre-death climbing behaviour. In the experiments performed with SeMNPV in the current thesis, light exposure was not needed during the period when the actual climbing took place. Infected larvae 'remembered' where the light came from and still moved to the original light source, even when in the dark. In nature, S. exigua larvae stay mostly at lower parts of plant foliage during day time (also when exposed to light from above) and climb up to feed during the night (Smits et al., 1987; Stapel et al., 1998). SeMNPV might make use of this already existing pattern in S. exigua larvae to induce tree-top disease. It is noticeable that light also plays a role in Bombyx mori nucleopolyhedrovirus (BmNPV)induced hyperactivity in the silkworm B. mori: the presence of light enhanced virus-induced hyperactivity. However, in this case light did not induce positive phototaxis in infected silkworms, since both virus- and mock-infected larvae showed similar levels of phototaxis (Kamita et al., 2005).

Though we observed that the viral egt gene and the timing of light played important roles in SeMNPV-induced tree-top disease, the question remains whether host pathway(s) are hijacked by SeMNPV to induce this behavioural change. Studies on how other parasites- induce phototaxis or geotaxis-related behaviour show a strong link with changes in the host CNS. For example, positive phototaxis is also observed in amphipods (Gammarus insensibilis) infected with trematodes (Microphallus papillorobustus). The expression of aromatic-L-amino acid decarboxylase, which is an enzyme involved in serotonin synthesis, was found to be significantly higher in infected G. insensibilis than in non-infected amphipods. Crickets infected with Gordian worms also present strong phototaxis behaviour compared to healthy crickets (Ponton et al., 2011). In manipulated crickets, the levels of proteins involved in CNS development, neurogenesis, circadian rhythm and neurotransmitter production were significantly higher than in healthy crickets (Biron et al., 2006). 
To identify which host molecules (in the CNS) are involved in SeMNPV-induced tree-top disease/phototaxis, we performed a transcriptomic study and compared the transcriptome of phototactic and non-phototactic larvae. Since light between 43-50 hpi was needed to induce positive phototaxis in SeMNPV-infected S. exigua larvae, samples were taken at different time points to include samples of infected larvae with and without phototactic behaviour. Larvae exposed to six different treatments were processed and included in an RNA-seq study. As shown in Figure 1, larvae of samples 1, 3, and 5 were kept under the normal $14 \mathrm{~h}$ light: $10 \mathrm{~h}$ dark photoperiod regime until 28, 46, and 54 hpi, respectively (samples 1, 3, 5, Figure 1); larvae of samples 2, 4, and 6 were kept under the normal $14 \mathrm{~L}$ : 10 D regime until 9, 35, and 35 hpi, respectively, after which they were kept in complete darkness (0 L: 24 D) until 28, 46, and 54 hpi, respectively (sample 2, 4, 6, Figure 1). Since mock-infected larvae developed faster than virus-infected larvae, samples were collected earlier, at $28 \mathrm{hpi}$, to ensure that the larvae used in the analysis were in the same physiological stage (late second instar). After these incubation periods, heads from infected larvae were collected for RNA extraction. The experiments were performed in three different weeks to obtain three biological replicates. Extracted RNA samples were sequenced by Illumina Hiseq 2500 and de novo assembly was performed to construct a reference genome. However, during downstream principal component analysis (PCA), which is used to check similarity and reproducibility among biological replicates, the results showed significant variation among biological replicates. Though we still performed a differential gene expression (DGE) analysis, no transcripts were found to be significantly different between phototactic and non-phototatic larvae. Future studies should use biological replicates collected at the same time point to avoid batch effects or an alternative analysis should be used to correct for the batch effect. 

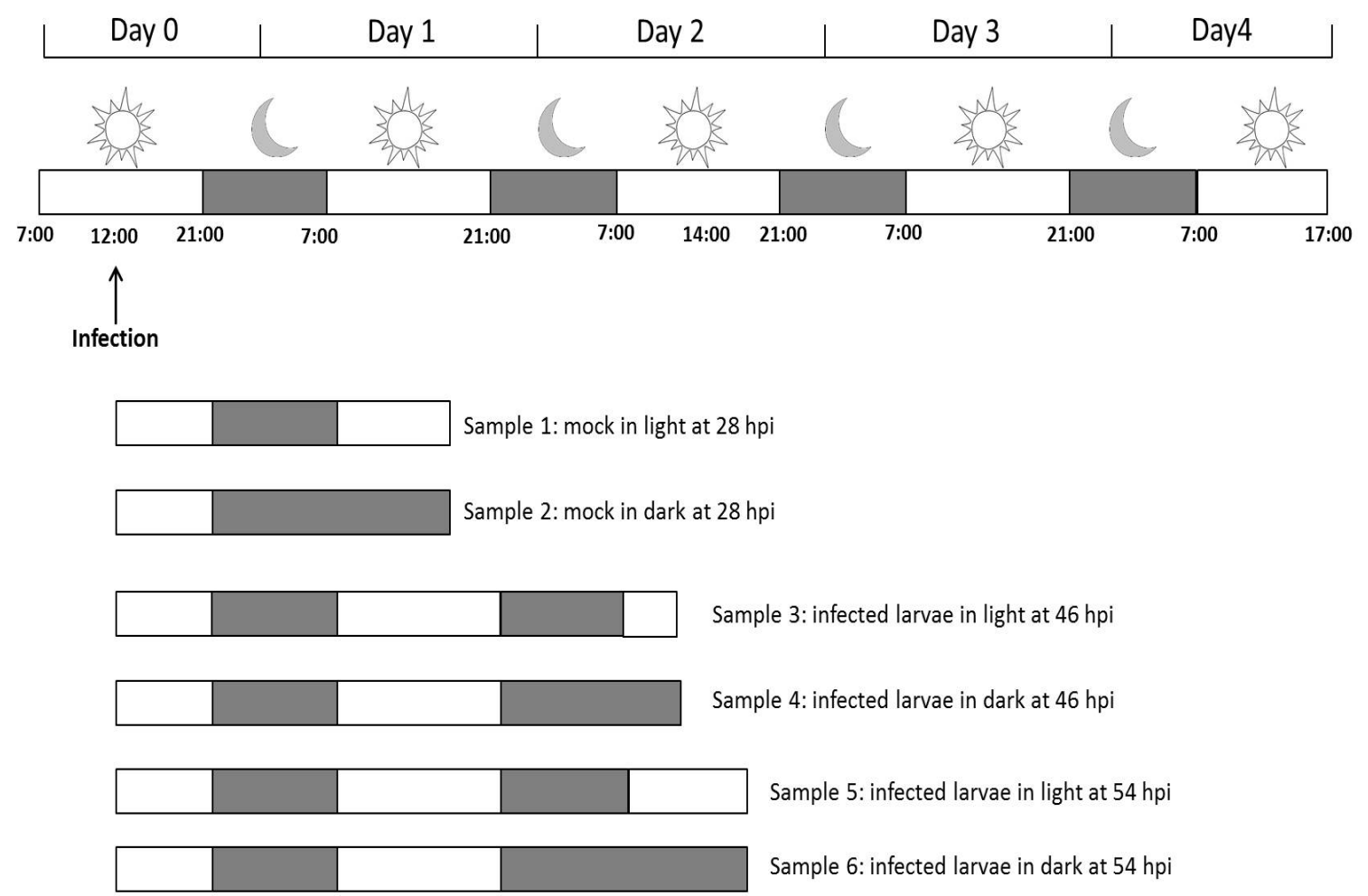

Figure 1. Scheme of the experimental set-up for RNA-seq sample preparation. Spodoptera exigua larvae were infected with wild type SeMNPV. The grey block represents a dark interval and the white block represents a light interval.

\section{The role of the baculovirus ptp2 gene in modulation of the host immune system}

Besides inducing behavioural manipulation, many viruses have developed alternative ways to enhance their transmission: host immune system modulation is one of these strategies (Anasir et al., 2017; Clem and Passarelli, 2013; Suderman et al., 2008). Apoptosis, or programmed cell death, normally is part of the insect immune response upon virus infection to suppress virus replication. However, many viruses have developed the ability to suppress host-induced apoptosis, by expressing anti-apoptotic genes (Clem and Passarelli, 2013; Perez-Chacon et al., 2014). On the other hand, some viruses have evolved to actively induce apoptosis to use the resulting apoptotic bodies as a mean of transport to enter other cells or to down regulate cellular immune responses by inducing apoptosis in specific cell types (Chitnis et al., 2011; SchultzCherry et al., 2001; Suderman et al., 2008). For example, the protein tyrosine phsphatase-H2 (ptp-h2) from the polydnavirus Microplitis demolitor bracovirus (MdBV) is responsible for induction of apoptosis in immune cells of Spodoptera frugiperda caterpillars, which contributes to host immunosuppression (Suderman et al., 2008). The iridovirus serine/threonine kinase (ISTK) from Chilo iridescent virus (CIV) induced apoptosis in cell lines derived from the spruce 
budworm Choristoneura fumiferana and the boll weevil Anthonomus grandis (Chitnis et al., 2011). In Chapter 4, the function of the baculovirus SeMNPV ptp2 gene (a homologue of the MdBV ptp-h2 gene) was investigated. The results showed that SeMNPV PTP2 induced mild apoptosis in Sf21 cells upon transient expression. The phosphatase activity of PTP2 was needed for this process. Moreover, deletion of the ptp2 gene from the SeMNPV genome prevented the induction of apoptosis in S. exigua hemocytes. Though deletion of the ptp2 gene did not affect virus titer and virulence indices (such as viral infectivity and infected host time to death), the occlusion body (OB) yield was significantly lower in $S$. exigua larvae infected by the mutant virus lacking the ptp2 gene than the yield in larvae infected by the WT virus. Therefore, we hypothesize that ptp2-induced apoptosis in hemocytes leads to suppression of the host immune system and eventually contributes to higher OB yields. The pro-apoptotic effect of SeMNPV PTP2 seems to be restricted to certain cell types and might be tissue specific. This is because expression of PTP2 did not induce apoptosis in other cell lines upon transient expression, including T. $n i$ High Five cells, Drosophila melanogaster S2 cells, and S. exigua Se301 cells. Whether SePTP2 induces apoptosis only in S. exigua larvae hemocytes, but not in other tissues/cell types needs further investigation. There is no explanation for why a viral gene induces apoptosis in one cell type, but not in another cell type. One possible reason is that certain regulatory pathways that are needed to trigger apoptosis are only active in specific cells. The situation becomes even more complex if one realizes that baculovirus genomes may contain both pro-apoptotic genes as well as anti-apoptotic genes (p35 or inhibitor of apoptosis (iap) genes).

Besides functioning as a typical PTP, SePTP2 possesses a C-terminal consensus sequence which is characteristic for mitogen-activated protein kinase (MAPK) phosphatases. MAPK phosphatases remove the phospho-residue from MAPKs. There are three major MAPKs active in various pathways: extracellular signal-regulated kinase (ERK), c-Jun N-terminal kinase (JNK) and p38 (Abdelwahid et al., 2007; Kim et al., 2008). All three MAPKs have been reported to be involved in inducing apoptosis (Abdelwahid et al., 2007; Kim et al., 2008). Moreover, the ERK- and JNK- dependent pathways contribute to higher BV and OB yields in BmNPV-infected larvae (Katsuma et al., 2007). Therefore, SePTP2 may contribute to higher OB yields in S. exigua larvae by functioning as a MAPK phosphatase that affects host MAPK pathways. To test our hypothesis, a similar proteomic approach as described in Chapter 6 was used to identify host proteins that interact with SePTP2. The SeMNPV ptp2 gene was first cloned fused to $g f p$ into a $\triangle p t p$ AcMNPV backbone to generate a recombinant virus. A co- 
immunoprecipitation assay using anti-GFP antibodies was performed to pull down GFP-tagged PTP2 proteins from virus-infected S. exigua larvae. Proteins that co-purified with PTP2 were identified with LC-MS/MS analysis. However, no host proteins with significantly higher levels in the PTP2-GFP sample group compared to the control group (which only expressed GFP) were found. This might be because SePTP2 was cloned into an AcMNPV background instead of its natural genetic background SeMNPV. Though SePTP2 was produced (data were not shown), it maybe not be able to execute its function. Future experiments should be repeated by expressing PTP2 in an SeMNPV background.

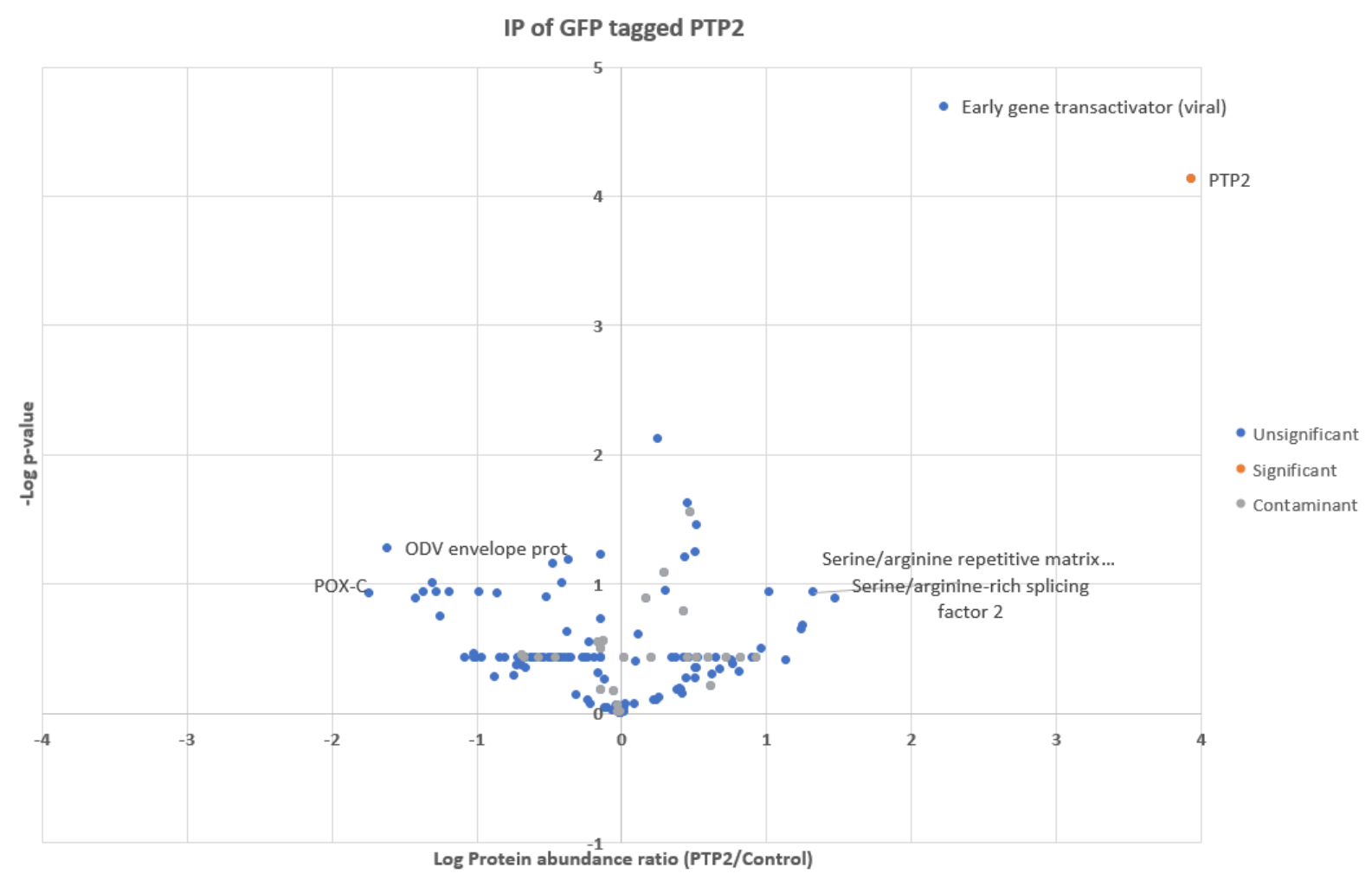

Figure 2: Mass spectrometric proteomic analysis of purified PTP2-GFP proteins. PTP2-GFP proteins were prepared from AcMNPV infected larvae and precipitated by GFP antiserum. The precipitation experiments were conducted three times from the same starting material and precipitated proteins were analysed with LC-MS/MS analysis using MaxQuant software. The statistical analysis was performed with the Perseus module. The Y-axis shows the relative abundance (peak height) as log (10) values, and $\mathrm{X}$-axis shows the $\log (10)$ of the abundance ratio of proteins precipitated in the PTP2-GFP group over proteins precipitated in the GFP control group. Proteins that were significantly enriched in the PTP2GFP sample over the GFP control are shown in orange $(\mathrm{P}<0.01)$. Except for PTP2, no proteins were significantly enriched in the PTP2-GFP sample. Proteins that are not significantly enriched are shown in blue. Contaminants such as keratins and trypsin are shown in grey. 
Homologues of the SeMNPV ptp2 gene are present in all subclades of baculoviruses, including the Group I NPV, Group II NPV and GVs (van Houte et al., 2012), but ptp2 is not present in all baculovirus genomes, and AcMNPV is an example of a virus that lacks ptp2 (Ayres et al., 1994). Whether the $p t p 2$ gene in other baculoviruses has a conserved role in inducing apoptosis needs further investigation. Besides a virus encoded enzyme, the PTP2 is a structural protein of Chrysodeixis chalcites NPV BV (Xu et al., 2011). Mamestra brassicae infected with $M$. brassicae MNPV dispersed over larger areas than uninfected individuals (Goulson, 1997; Vasconcelos, 1996). However, MbMNPV (although a Group I NPV) does not contain a ptp gene, such as AcMNPV and BmNPV (see below). In contrast, MbNPV has a ptp2 homologue in its genome, but the possible function of $p t p 2$ in behavioural manipulation remains to be determined. In conclusion, PTP2 may have more functions than we currently know, as was also found for baculovirus-encoded PTP (Chapter 6).

\section{Further insights into baculovirus ptp-induced hyperactivity}

The second type of behavioural manipulation induced by baculoviruses is hyperactivity. Infected hosts wander over larger areas, which is thought to spread virus progeny over a further distance (Goulson, 1997; Kamita et al., 2005). Kamita et al. (2005) first identified that the ptp gene from BmNPV is involved in triggering hyperactivity in B. mori larvae. Previous research in the laboratory also identified that the $p t p$ gene from AcMNPV is involved in inducing hypermobility in S. exigua larvae (van Houte et al., 2012). However, the exact mechanism exerted by the $p t p$ gene seems to be different between these two systems. In BmNPV-infected B. mori larvae, the phosphatase activity of encoded PTP is not needed for behavioural manipulation (Kamita et al., 2005). On the contrary, in AcMNPV-infected S. exigua larvae, the phosphatase activity was required to trigger hyperactivity, because mutation of the catalytic site of AcPTP blocked induction of hyperactivity (van Houte et al., 2012).

The involvement of AcPTP phosphatase activity in inducing hyperactivity suggests that host or viral proteins serve as substrate(s) for the AcMNPV PTP enzyme and that dephosphorylation of the substrate(s) eventually leads to altered host behaviour. In Chapter 6, we used a proteomic approach to identify viral and host proteins that interact with AcPTP. The results showed that AcPTP may have more functions than we currently know: AcPTP potentially is involved in nucleocapsid assembly, nucleocapsid transport, ODV envelopment and virus dissemination. There is no doubt that phosphorylation and dephosphorylation play important roles during 
baculovirus infection (Cheley et al., 1992; Funk and Consigli, 1993). Therefore, it is reasonable that AcPTP have multiples roles in the baculovirus replication cycle.

Since locomotion behaviour is primarily controlled by the CNS (Strauss, 2002), induction of hyperactivity is likely to have its basis in the CNS. Whether AcPTP affects the host CNS was not studied in this thesis. In BmNPV-infected B. mori, BmPTP appears to have a role as a structural protein, that allows higher virus titer in the brain. This conclusion was drawn mainly because Western blot results showed that BmPTP was primarily localized in the BV envelope (Katsuma et al., 2012). Moreover, BmPTP showed strong interaction with the viral phosphoprotein 78/83 (PP78/83) in a yeast-2-hybrid assay. PP78/83 encodes a WASP-like protein that localizes at one end of the nucleocapsid structure. The C-terminal end of PP78/83 showed a strong interaction with BmPTP. Moreover, deletion of the $p t p$ gene from the BmNPV genome affected BV production in many tissues including the larval brain. Reduced BV titers in the larval brain might be the reason why hyperactivity was blocked in $\Delta p t p$ BmNPV-infected B. mori larvae. Previous research has reported that AcPTP is associated with both BVs and ODVs (Li and Miller, 1995). In our study, AcPTP did not co-purify with PP78/83 directly, however, the co-purified proteins BV/ODV-C42 and VP1054 have been reported to interact with PP78/83 (Li et al., 2010), suggesting that AcPTP also functions as a structural protein. Our previous study showed that deletion of the ptp gene did not affect AcMNPV virulence indices, including the viral infectivity and infected host time to death (van Houte et al., 2012), however, the effect on virus titer in different tissues might have been overlooked. A future study is needed to find out whether deletion of the ptp gene affects the AcMNPV virus titer in the S. exigua larval brain, which might lead to a reduction in hyperactivity. One host protein that co-purified with AcPTP was lamin C. Lamins have been reported to play an important function in remodeling the nuclear architecture (Schulze et al., 2009; Young et al., 2012), which may help to facilitate egress of nucleocapsids from the nucleus. Lamins play crucial roles in brain development and defects in lamins cause an array of (mental) disorders in humans and rats (Schulze et al., 2009; Young et al., 2012) Lamins in the insect brain may have similar functions. The interaction of AcPTP with lamins may therefore also allow the virus to invade the larval brain..

It is hypothesized that AcMNPV induces behavioural changes via affecting the S. exigua CNS, however, little is known on the morphology and function of different parts of the larval brain. Therefore, in Chapter 7 a 3D model of the S. exigua larval brain was constructed based on immunolabelling of serotonin. The model describes the brain morphology and the position of 
identified neuropil supercategories in the brain. The distribution of serotonergic neurons in the larval brain was also analysed, including the location and the number of serotonergic neuron cells in each serotonergic neuron cell cluster. Confocal and electron microscopy analysis confirmed that AcMNPV is present and replicates in the larval brain: AcMNPV started to infect the host brain at 2 days post infection (dpi) and the brains of most caterpillars were infected at 3 dpi. Moreover, it seems that the viruses only invade specific regions of the CNS. Chapter 7 is only the start of revealing how baculovirus infection in the brain may lead to behavioural manipulation. More research is definitely needed to find out i) whether AcMNPV infects (or affects) biogenic amines-secreting cells in the CNS; ii) whether the ptp gene and the phosphatase activity of the encoded protein controlls virus entry into the brain; iii) which (molecular) pathways are involved in the brain to induce hyperactivity.

\section{Where do we stand now?}

Host manipulation by baculoviruses is the outcome of a highly complex interaction between the virus and the host and depends on a series of events in a spatial and temporal order. Knowledge gained from this thesis is not enough to understand the whole process of (behavioural) manipulation but provides fundamental insight into the underlying mechanisms.

Two baculovirus genes (the $p t p$ gene and the egt gene) have been studied extensively for their role in host behavioural manipulation. It is clear that there is no general mechanism that baculoviruses use to induce behavioural manipulation. EGT does not have a conserved role in inducing tree-top disease: it is needed in SeMNPV- and LdMNPV-induced tree-top disease, but not involved in AcMNPV-induced tree-top disease. The importance of environmental conditions in behavioural manipulation might be overlooked in many cases. In SeMNPVinduced tree-top disease, the direction and the timing of light both play vital important roles. Therefore, small alterations in environment conditions might change the outcome of behavioural manipulation completely.

Even though the role of $p t p$ is conserved in AcMNPV- and BmNPV-induced hyperactivity, differences seem to exist in the downstream pathways. Proteomic analysis of PTP substrates revealed that PTP may have more functions that we currently know. The involvement of a viral gene in behavioural manipulation does not necessarily mean that the alteration is directly induced by this gene. Besides the behavioural manipulation of the egt gene, the $p t p 2$ gene from SeMNPV is involved in inducing apoptosis to manipulate the host immune system. Both types 
of manipulation are regarded as actions taken by the virus to enhance transmission to new host: either by spreading virus progeny over larger areas or by producing more progeny OBs.

Host molecules that are involved in baculovirus-induced behavioural manipulation are still largely unknown. Comparing the transcriptomes or proteomes of manipulated and unmanipulated hosts will provide more insight into this process. Currently, only few lepidopteran genomes have been sequenced. Therefore, sequencing more host genomes (including $S$. exigua) is highly desired. Baculoviruses most likely induce behavioural manipulation by invading and subsequently affecting the host CNS. We have described the brain morphology and serotonergic neuron distribution in the larval brain. We also demonstrated the presence of AcMNPV in the larval brain. The knowledge obtained in this thesis on baculovirus-induced behavioural manipulation provides useful information to start more in-depth studies in this field. 


\section{References}

Abdelwahid E, Yokokura T, Krieser RJ, Balasundaram S, Fowle WH, White K, 2007. Mitochondrial Disruption in Drosophila Apoptosis. Developmental Cell 12, 793-806.

Adamo SA, 2002. Modulating the modulators: parasites, neuromodulators and host behavioral change. Brain, behavior and evolution 60, 370-377.

Adamo SA, 2008. Bidirectional connections between the immune system and the nervous system in insects. In Beckage NE (ed) Insect immunology. Academic, San Diego, 129-149.

Adamo SA, 2014. Parasitic aphrodisiacs: manipulation of the hosts' behavioral defenses by sexually transmitted parasites. Integrative and Comparative Biology 54, 159-165.

Adamo SA, Bartlett A, Le J, Spencer N, Sullivan K, 2010. Illness-induced anorexia may reduce trade-offs between digestion and immune function. Animal Behaviour 79, 3-10.

Adamo SA, Kovalko I, Easy RH, Stoltz D, 2014. A viral aphrodisiac in the cricket Gryllus texensis. Journal of Experimental Biology 217, 1970-1976.

Airenne KJ, Peltomaa E, HytoÈnen VP, Laitinen OH, Ylä-Herttuala S, 2003. Improved generation of recombinant baculovirus genomes in Escherichia coli. Nucleic Acids Research 31, 101.

Anasir MI, Baxter AA, Poon IKH, Hulett MD, Kvansakul M, 2017. Structural and functional insight into canarypox virus CNP058 mediated regulation of apoptosis. Viruses 9, 305.

Ando H, Kuwasawa K, 2004. Neuronal and neurohormonal control of the heart in the stomatopod crustacean, Squilla oratoria. Journal of Experimental Biology 207, 4663-4677.

Aubin-Horth N, Renn SC, 2009. Genomic reaction norms: using integrative biology to understand molecular mechanisms of phenotypic plasticity. Molecular Ecology 18, 3763-3780.

Audsley N, Duve H, Thorpe A, Weaver RJ, 2000. Morphological and physiological comparisons of two types of allatostatin in the brain and retrocerebral complex of the tomato moth, Lacanobia oleracea (Lepidoptera: Noctuidae). Journal of Comparative Neurology 424, 37-46.

Ayres MD, Howard SC, Kuzio J, Lopez-Ferber M, Possee RD, 1994. The complete DNA sequence of Autographa californica nuclear polyhedrosis virus. Virology 202, 586-605.

Azidah AA, Sofian-Azirun M, 2006. Life history of Spodoptera exigua (Lepidoptera: Noctuidae) on various host plants. Bulletin of Entomological Research 96, 613-618.

Ball BV, 1989. Varroa jacobsoni as a virus vector. In Cavalloro R (ed) Present status of varroatosis in Europe and progress in the Varroa mite control. Office Official Publications EC, Luxembourg, 241-244.

Ban L, Ahmed E, Ninkovic V, Delp G, Glinwood R, 2008. Infection with an insect virus affects olfactory behaviour and interactions with host plant and natural enemies in an aphid. Entomologia Experimentalis Et Applicata 127,108-117

Baracchi D, Fadda A, Turillazzi S, 2012. Evidence for antiseptic behaviour towards sick adult bees in honey bee colonies. Journal of Insect Physiology 58, 1589-1596.

Bartel DP, 2004. MicroRNAs: genomics, biogenesis, mechanism, and function. Cell 116, 281-297.

Belyavskyi M, Braunagel SC, Summers MD, 1998. The structural protein ODV-EC27 of Autographa californica nucleopolyhedrovirus is a multifunctional viral cyclin. Proceedings of the National Academy of Sciences of the United States of America 95, 11205-11210.

Benesh DP, Duclos LM, Nickol BB, 2005. The behavioral response of amphipods harboring Corynosoma constrictum (Acanthocephala) to various components of light. Journal of Parasitiology 91, 731-736.

Bennett KE, Hopper JE, Stuart MA, West M, Drolet BS, 2008. Blood-feeding behavior of vesicular stomatitis virus infected Culicoides sonorensis (Diptera: Ceratopogonidae). Journal of Medical Entomology 45, 921-926. 
Bianchi FJ, Snoeijing I, van der Werf W, Mans RM, Smits PH, Vlak JM, 2000. Biological activity of SeMNPV, AcMNPV, and three AcMNPV deletion mutants against Spodoptera exigua larvae (Lepidoptera: Noctuidae). Journal of Invertebrate Pathology 75, 28-35.

Bielow C, Mastrobuoni G, Kempa S, 2016. Proteomics quality control: quality control software for MaxQuant results. Journal of Proteome Research 15, 777-787.

Biron D, Ponton F, MarcheL, Galeotti N, Renault L, Demey-Thomas E, Poncet J, Brown S, Jouin P, Thomas F, 2006. 'Suicide' of crickets harbouring hairworms: a proteomics investigation. Insect Molecular Biology 15, 731-742.

Biron DG, Loxdale HD, 2013. Host-parasite molecular cross-talk during the manipulative process of a host by its parasite. Journal of Experimental Biology 216, 148-160.

Biron DG, Marche L, Ponton F, Loxdale HD, Galeotti N, Renault L, Joly C, Thomas F, 2005. Behavioural manipulation in a grasshopper harbouring hairworm: a proteomics approach. Proceedings of the Royal Society of London B: Biological Sciences 272, 2117-2126.

Biron DG, Ponton F, Marche L, Galeotti N, Renault L, Demey-Thomas E, Poncet J, Brown SP, Jouin P, Thomas F, 2006. 'Suicide' of crickets harboring hairworms: a proteomics investigation. Insect Molecular Biology 15, 731-742.

Boleli I, Hartfelder K, Simoes Z, 1995. Serotonin-like immunoreactivity in the central nervous and neuroendocrine system of honey-bee (Apis mellifera) larvae and pupae. Zoology Analysis of Complex Systems 99, 58-67.

Bos N, Lefèvre T, Jensen AB, d'Ettorre P, 2012. Sick ants become unsociable. Journal of Evolutionary Biology 25, 342-351.

Braconi CT, Ardisson-Araújo DMP, Leme AFP, Oliveira JVdC, Pauletti BA, Garcia-Maruniak A, Ribeiro BM, Maruniak JE, Zanotto PMdA, 2014. Proteomic analyses of baculovirus Anticarsia gemmatalis multiple nucleopolyhedrovirus budded and occluded virus. Journal of General Virology 95, 980-989.

Braunagel SC, Guidry PA, Rosas-Acosta G, Engelking L, Summers MD, 2001. Identification of BV/ODV-C42, an Autographa californica nucleopolyhedrovirus orf101-encoded structural protein detected in infected-cell complexes with ODVEC27 and p78/83. Journal of Virology 75, 12331-12338.

Braunagel SC, He H, Ramamurthy P, Summers MD, 1996. Transcription, translation, and cellular localization of three Autographa californicanuclear polyhedrosis virus structural proteins: ODV-E18, ODV-E35, and ODV-EC27: ODVE18, ODV-E35, and ODV-EC27. Virology 222,100-114.

Burand JP, 2009. The sexually transmitted insect virus, Hz-2 V. Virologica Sinica 24, 428-435.

Burand JP, Rallis CP, Tan W, 2004. Horizontal transmission of Hz-2 V by virus infected Helicoverpa zea moths. Journal of Invertebrate Pathology 85, 128-131.

Burand JP, Tan W, 2006. Mate preference and mating behavior of male Helicoverpa zea (Lepidoptera: Noctuidae) infected with the sexually transmitted insect virus Hz-2 V. Annals of the Entomological Society of America 99, 969-973.

Burand JP, Tan W, Kim W, Nojima S, Roelofs W, 2005. Infection with the insect virus Hz-2v alters mating behavior and pheromone production in female Helicoverpa zea moths. Journal of Insect Science 5, 1-6.

Cai Y, Cheng Z, Li C, Wang F, Li G, Pang Y, 2010. Biological activity of recombinant Spodoptera exigua multicapsid nucleopolyhedrovirus against Spodoptera exigua larvae. Biological Control 55,178-185.

Cantera R, Thompson KS, Hallberg E, Nassel DR, Bacon JP, 1995. Migration of neurons between ganglia in the metamorphosing insect nervous system. Roux's Archives of Developmental Biology 205, 10-20.

Carballo A, Murillo R, Jakubowska A, Herrero S, Williams T, Caballero P, 2017. Co-infection with iflaviruses influences the insecticidal properties of Spodoptera exigua multiple nucleopolyhedrovirus occlusion bodies: Implications for the production and biosecurity of baculovirus insecticides. PLoS One 12:e177301.

Carinhas N, Robitaille AM, Moes S, Carrondo MJT, Jenoe P, Oliveira R, Alves PM, 2011. Quantitative proteomics of Spodoptera frugiperda cells during growth and baculovirus infection. PLoS One 6:e26444.

Castrejon F and Rojas JC, 2010. Behavioral responses of larvae and adults of Estigmene acrea (Lepidoptera: Arctiidae) to light of different wavelengths. Florida Entomologist 93, 505-509. 
Cézilly F, Thomas F, Médoc V, Perrot-Minnot M-J, 2010. Host-manipulation by parasites with complex life cycles: adaptive or not? Trends in Parasitology 26, 311-317

Chapman AD, 2006. Numbers of living species in Australia and the World. Australian Biological Resources Study, Canberra.

Charlton CA, Volkman LE, 1993. Penetration of Autographa californica nuclear polyhedrosis virus nucleocapsids into IPLB Sf 21 cells induces actin cable formation. Virology 197, 245-254.

Cheley S, Kosik KS, Paskevich P, Bakalis S, Bayley H, 1992. Phosphorylated baculovirus p10 is a heat-stable microtubuleassociated protein associated with process formation in Sf9 cells. Journal of Cell Science 102, 739-752.

Chitnis NS, Paul ER, Lawrence PK, Henderson CW, Ganapathy S, Taylor PV, Virdi KS, D'Costa SM, May AR, Bilimoria SL, 2011. A virion-associated protein kinase induces apoptosis. Journal of Virology, 85, 13144-13152.

Chung TY, Sun PF, Kuo JI, Lee YI, Lin CC, Chou JY, 2017. Zombie ant heads are oriented relative to solar cues. Fungal Ecology 25, 22-28.

Clarke P, Tyler KL, 2009. Apoptosis in animal models of virus-induced disease. Nature Reviews Microbiology 7, 144-155.

Clem RJ, 2001. Baculoviruses and apoptosis: the good, the bad, and the ugly. Cell Death \& Differentiation 8,137-143.

Clem RJ, 2005. The Role of apoptosis in defence against baculovirus infection in insects. In Griffin DE, editor. Role of Apoptosis in Infection Berlin, Heidelberg, Springer Berlin Heidelberg, Germany. p. 113-129.

Clem RJ, Fechheimer M, Miller LK, 1991. Prevention of apoptosis by a baculovirus gene during infection of insect cells. Science 254,1388-1390.

Clem RJ, Passarelli AL, 2013. Baculoviruses: sophisticated pathogens of insects. PLoS Pathogens 9, e1003729.

Clément N, Grieger JC, 2016. Manufacturing of recombinant adeno-associated viral vectors for clinical trials. Molecular Therapy-Methods \& Clinical Development 3, 16002.

Cohen DPA, Marek M, Davies BG, Vlak JM, van Oers MM, 2009. Encyclopedia of Autographa californica nucleopolyhedrovirus genes. Virologica Sinica 24, 359-414.

Condreay JP, Kost TA, 2007. Baculovirus expression vectors for insect and mammalian cells. Current Drug Targets 8, 11261131.

Cory J, Clarke E, Brown M, Hails R, O'Reilly D, 2004. Microparasite manipulation of an insect: the influence of the egt gene on the interaction between a baculovirus and its lepidopteran host. Functional Ecology 18, 443-450.

Cory JS, 2003. Ecological impacts of virus insecticides: host range and non-target organisms. In Hokkanen HMT, Hajek AE, editors. Environmental Impacts of Microbial Insecticides: Need and Methods for Risk Assessment Dordrecht. Springer Netherlands. p. 73-91.

Cory JS, Hoover K, 2006. Plant-mediated effects in insect-pathogen interactions. Trends in Ecology \& Evolution 21, 278-286

Cranston PS, 2010. Insect biodiversity and conservation in Australasia. Annual Review of Entomology 55, 55-75.

Danquah JO, Botchway S, Jeshtadi A, King LA, 2012. Direct interaction of baculovirus capsid proteins VP39 and EXON0 with kinesin- 1 in insect cells determined by fluorescence resonance energy transfer-fluorescence lifetime imaging microscopy. Journal of Virology 86, 844-853.

Dawkins R, 1976. The Selfish Gene. Oxford University Press. New York, USA.

Dawkins R, 1982. The Extended Phenotype: The Long Reach of the Gene. Oxford University Press. New York, USA.

de Bekker C, Merrow M, Hughes DP, 2014. From behavior to mechanisms: an integrative approach to the manipulation by a parasitic fungus (Ophiocordyceps unilateralis s.l.) of its host ants (Camponotus spp.). Integrative and Comparative Biology 54, 166-176.

de Bekker C, Ohm RA, Loreto RG, Sebastian A, Albert I, Merrow M, Brachmann A, Hughes DP, 2015. Gene expression during zombie ant biting behavior reflects the complexity underlying fungal parasitic behavioral manipulation. $B M C$ Genomics 16, 620 .

de Bekker C, Will I, Hughes DP, Brachmann A, Merrow M, 2017. Daily rhythms and enrichment patterns in the transcriptome of the behavior-manipulating parasite Ophiocordyceps kimflemingiae. PLoS ONE 12:e187170. 
Dechat T, Adam SA, Taimen P, Shimi T, Goldman RD, 2010. Nuclear lamins. Cold Spring Harbor Perspectives in Biology 2:a000547.

Dheilly NM, Maure F, Ravallec M, Galinier R, Doyon J, Duval D, Leger L, Volkoff AN, Misse D, Nidelet S, Demolombe V, Brodeur J, Gourbal B, Thomas F, Mitta G, 2015. Who is the puppet master? Replication of a parasitic wasp-associated virus correlates with host behaviour manipulation. Proceedings of the Royal Society B: Biological Sciences 282, 20142773.

Diz AP, Martinez-Fernandez M, Rolan-Alvarez E, 2012. Proteomics in evolutionary ecology: linking the genotype with the phenotype. Molecular Ecology 21, 1060-1080.

El Jundi B, Huetteroth W, Kurylas AE, Schachtner J, 2009. Anisometric brain dimorphism revisited: implementation of a volumetric 3D standard brain in Manduca sexta. Journal of Comparative Neurology 517, 210-225.

Elmore S, 2007. Apoptosis: a review of programmed cell death. Toxicologic Pathology 35, 495-516.

Elvira S, Gorria N, Munoz D, Williams T, Caballero P, 2010. A simplified low-cost diet for rearing Spodoptera exigua (Lepidoptera: Noctuidae) and its effect on S. exigua nucleopolyhedrovirus production. Journal of Economic Entomology 103, 17-24.

Entwistle PF, Forkner AC, Green BM, Cory JS, 1993. Avian dispersal of nuclear polyhedrosis viruses after induced epizootics in the pine beauty moth, Panolis flammea (Lepidoptera: Noctuidae). Biological Control 3,61-69.

Evans HF, Allaway GP, 1983. Dynamics of baculovirus growth and dispersal in Mamestra brassicae L. (Lepidoptera: Noctuidae) larval populations introduced into small cabbage plots. Applied and Environmental Microbiology 45, 493-501.

Fedorka KM, Mousseau TA, 2007. Immune system activation affects male sexual signal and reproductive potential in crickets. Behaviour Ecology 18, 231-235.

Feng G, Yu Q, Hu C, Wang Y, Yuan G, Chen Q, Yang K, Pang Y, 2007. Apoptosis is induced in the haemolymph and fat body of Spodoptera exigua larvae upon oral inoculation with Spodoptera litura nucleopolyhedrovirus. Journal of General Virology 88, 2185-2193.

Fereres A, Shukle RH, Araya JE, Foster JE, 1990. Probing and feeding behavior of Sitobion avenae (F.) (Horn., Aphididae) on three wheat cultivars infected with barley yellow dwarf virus. Journal of Applied Entomology 109, 29-36

Fieber LA, 2017. Neurotransmitters and neuropeptides of invertebrates. Byrne JH, Editor. The Oxford Handbook of Invertebrate Neurobiology.

Fischbach KF, 1979. Simultaneous and successive color contrast expressed in "slow" phototactic behavior of walking Drosophila melanogaster. Journal of Comparative Physiology B: Biochemical, Systemic, and Environmental Physiology 130, 161-171.

Fitzpatrick MJ, Ben-Shahar Y, Smid HM, Vet LE, Robinson GE, Sokolowski MB, 2005. Candidate genes for behavioural ecology. Trends in Ecology \& Evolution 20, 96-104.

Flipsen J, Mans R, Kleefsman A, Knebel-Mörsdorf D, Vlak JM, 1995. Deletion of the baculovirus ecdysteroid UDPglucosyltransferase gene induces early degeneration of Malpighian tubules in infected insects. Journal of Virology 69, 4529-4532.

Fros JJ, Liu WJ, Prow NA, Geertsema C, Ligtenberg M, Vanlandingham DL, Schnettler E, Vlak JM, Suhrbier A, Khromykh AA, Pijlman GP, 2010. Chikungunya virus nonstructural protein 2 inhibits type I/II interferon-stimulated JAK-STAT signaling. Journal of Virology 84, 10877-10887.

Fujiyuki T, Takeuchi H, Ono M, Ohka S, Sasaki T, Nomoto A, Kubo T, 2004. Novel insect Picorna-like virus identified in the brains of aggressive worker honeybees. Journal of Virology 78, 1093-1100.

Fujiyuki T, Takeuchi H, Ono M, Ohka S, Sasaki T, Nomoto A, Kubo T, 2005. Kakugo virus from brains of aggressive worker honeybees. Advanced Virus Research 65, 1-27.

Funk CJ, Consigli RA, 1993. Phosphate cycling on the basic protein of Plodia interpunctella granulosis virus. Virology 193 , 396-402. 
Gabitzsch ES, Blair CD, Beaty BJ, 2006. Effect of La Crosse Virus infection on insemination rates in female Aedes triseriatus (Diptera: Culicidae). Journal of Medical Entomology 43, 850-852.

Gandon S, Rivero A, Varaldi J, 2006. Superparasitism evolution: adaptation or manipulation? American Naturalist 167, 1-22 Gandon S, Varaldi J, Fleury F, Rivero A, 2009. Evolution and manipulation of parasitoid egg load. Evolution 63, 2974-2984.

Gisder S, Aumeier P, Genersch E, 2009. Deformed wing virus: replication and viral load in mites (Varroa destructor). Journal of General Virology 90, 463-467.

Goertz GP, Fros JJ, Miesen P, Vogels CB, van der Bent ML, Geertsema C, Koenraadt CJ, van Rij RP, van Oers MM, Pijlman GP, 2016. Noncoding subgenomic flavivirus RNA is processed by the mosquito RNA interference machinery and determines West Nile Virus transmission by Culex pipiens mosquitoes. Journal of Virology 90, 10145-10159.

Golikhajeh N, Naseri B, Razmjou J, 2017. Geographic Origin and Host Cultivar Influence on Digestive Physiology of Spodoptera exigua (Lepidoptera: Noctuidae) Larvae. Journal of Insect Science 17, 12.

Gouin A, Bretaudeau A, Nam K., Gimenez S., Aury J M, Duvic B, ... \& Kuwar S, 2017. Two genomes of highly polyphagous lepidopteran pests (Spodoptera frugiperda, Noctuidae) with different host-plant ranges. Scientific Reports 7, 11816.

Goulson D, 1997. Wipfelkrankheit: modification of host behaviour during baculoviral infection. Oecologia 109, 219-228.

Graumann PL, 2001. SMC proteins in bacteria: condensation motors for chromosome segregation? Biochimie 83, 53-59.

Grimstad PR, Ross QE, Craig GB Jr, 1980. Aedes triseriatus (Diptera: Culicidae) and La Crosse virus. II. Modification of mosquito feeding behavior by virus infection. Journal Medical Entomology 17,1-7.

Guan Z, Zhong L, Li C, Wu W, Yuan M, Yang K, 2016. The Autographa californica multiple nucleopolyhedrovirus ac54 gene is crucial for localization of the major capsid protein VP39 at the site of nucleocapsid assembly. Journal of Virology 90, 4115-4126.

Hamblin S, Tanaka M, 2013. Behavioural manipulation of insect hosts by Baculoviridaeas a process of niche construction. BMC Evolution Biology 13, 170.

Hamm JJ, Carpenter JE, Styer EL, 1996. Oviposition day effect on incidence of agonadal progeny of Helicoverpa zea (Lepidoptera: Noctuidae) infected with a virus. Annals of the Entomological Society of America 89, 266-275

Han JY, Miller SA, Wolfe TM, Pourhassan H, Jerome KR, 2009. Cell type-specific induction and inhibition of apoptosis by Herpes Simplex virus type 2 ICP10. Journal of Virology 83, 2765-2769.

Han Y, van Houte S, Drees GF, van Oers MM, Ros VID, 2015. Parasitic manipulation of host behaviour: baculovirus SeMNPV EGT facilitates tree-top disease in Spodoptera exigua larvae by extending the time to death. Insects 6, 716-731.

Han Y, van Houte S, van Oers MM, Ros VID, 2018. Baculovirus PTP2 functions as a pro-apoptotic protein. Viruses 10, 181. Han Y, van Oers MM, van Houte S, Ros VID, 2015b. Virus-induced behavioural changes in insects. In Mehlhorn H, editor. Host Manipulations by Parasites and Viruses. Cham: Springer International Publishing. p. 149-174.

Hara K, Funakoshi M, Kawarabata T, 1995. A cloned cell line of Spodopteta exigua has a highly increased susceptibility to the Spodoptera exigua nuclear polyhedrosis virus. Canadian Journal of Microbiology 41, 1111-1116.

Harris JW, 2007. Bees with Varroa Sensitive Hygiene preferentially remove mite infested pupae aged $\leq$ five days post capping. Journal of Apicultural Research 46, 134-139.

Hegedus D, Erlandson M, Gillott C, Toprak U, 2009. New insights into peritrophic matrix synthesis, architecture, and function. Annual Review of Entomology 54, 285-302.

Herniou EA, Luque T, Chen X, Vlak JM, Winstanley D, Cory JS, O'Reilly DR, 2001. Use of whole genome sequence data to infer baculovirus phylogeny. Journal of Virology 75, 8117-8126.

Herniou EA, Olszewski JA, Cory JS, O'Reilly DR, 2003. The genome sequence and evolution of baculoviruses. Annual Review of Entomology 48, 211-234.

Herz A, Kleespies RG, Huber J, Chen X, Vlak JM, 2003. Comparative pathogenesis of the Helicoverpa armigera singlenucleocapsid nucleopolyhedrovirus in noctuid hosts of different susceptibility. Journal of Invertebrate Pathology $83: 31-36$. 
Hofmann, 1891. Die Schlaffsucht (Flacherie) der Nonne (Liparis monacha): Nebst e. Anh. Vortr. über Insektentötende Pilze: Weber, Frankfurt, Germany.

Hoover K, Grove M, Gardner M, Hughes DP, McNeil J, Slavicek J, 2011. A gene for an extended phenotype. Science 333, 1401.

Hu Z, Zhao H, Thieme T, 2013. Modification of non-vector aphid feeding behavior on virus-infected host plant. Journal of Insect Science 13, 1-11.

Huetteroth W, Jundi B, Jundi S, Schachtner J, 2010. 3D-reconstructions and virtual 4D-visualization to study metamorphic brain development in the Sphinx moth Manduca Sexta. Frontiers in Systems Neuroscience 4, 7.

Hughes D, 2013. Pathways to understanding the extended phenotype of parasites in their hosts. Journal of Experimental Biology 216, 142-147.

Hughes DP, Andersen SB, Hywel-Jones NL, Himaman W, Billen J, Boomsma JJ, 2011. Behavioral mechanisms and morphological symptoms of zombie ants dying from fungal infection. BMC Ecology 11, 173.

Huser A, Rohwedder A, Apostolopoulou AA, Widmann A, Pfitzenmaier JE, Maiolo EM, Selcho M, Pauls D, von Essen A, Gupta T, Sprecher SG, Birman S, Riemensperger T, Stocker RF, Thum AS, 2012. The serotonergic central nervous system of the Drosophila larva: Anatomy and behavioral function. PLOS ONE 7, e47518.

IBM SPSS Statistics for Windows, Version 22.0. Armonk, NY, IBM Corp.

Igney FH, Krammer PH, 2002. Death and anti-death: tumour resistance to apoptosis. Nature Reviews Cancer 2, 277-288.

IJkel WFJ, van Strien EA, Heldens JGM, Broer R, Zuidema D, Goldbach RW, Vlak JM, 1999. Sequence and organization of the Spodoptera exigua multicapsid nucleopolyhedrovirus genome. Journal of General Virology 80, 3289-3304.

Ingwell LL, Eigenbrode SD, Bosque-Perez NA, 2012. Plant viruses alter insect behavior to enhance their spread. Scientific Reports 2, 578.

Iqbal J, Mueller U, 2007. Virus infection causes specific learning deficits in honeybee foragers. Proceedings of the Royal Society B: Biological Sciences 274, 1517-1521.

Ito K, Shinomiya K, Ito M, Armstrong JD, Boyan G, Hartenstein V, Harzsch S, Heisenberg M, Homberg U, Jenett A, 2014. A systematic nomenclature for the insect brain. Neuron 81, 755-765.

Jackson BT, Brewster CC, Paulson SL, 2012. La Crosse virus infection alters blood feeding behavior in Aedes triseriatus and Aedes albopictus (Diptera: Culicidae). Journal of Medical Entomology 49, 1424-1429.

Jacot A, Scheuber H, Brinkhof MW, 2004. Costs of an induced immune response on sexual display and longevity in field crickets. Evolution 58, 2280-2286.

Jakob NJ, Kleespies RG, Tidona CA, Müller K, Gelderblom HR, Darai G, 2002. Comparative analysis of the genome and host range characteristics of two insect iridoviruses: Chilo iridescent virus and a cricket iridovirus isolate. Journal of General Virology 83, 463-470.

Jaworska J, Coulombe F, Downey J, Tzelepis F, Shalaby K, Tattoli I, Berube J, Rousseau S, Martin JG, Girardin SE, McCullers JA, Divangahi M, 2014. NLRX1 prevents mitochondrial induced apoptosis and enhances macrophage antiviral immunity by interacting with influenza virus PB1-F2 protein. Proceedings of the National Academy of Sciences of the United States of America 111, 2110-2119.

Jehle JA, Blissard GW, Bonning BC, Cory JS, Herniou EA, Rohrmann GF, Theilmann DA, Thiem SM, Vlak JM, 2006. On the classification and nomenclature of baculoviruses: a proposal for revision. Archives of Virology 151,1257-1266.

Jenkins DA, Hunter WB, Goenaga R, 2011. Effects of invertebrate iridescent virus 6 in Phyllophaga vandinei and its potential as a biocontrol delivery system. Journal of Insect Science 11, 44.

Jia Z, Barford D, Flint AJ, Tonks NK, 1995. Structural basis for phosphotyrosine peptide recognition by protein tyrosine phosphatase 1B. Science 268,1754-1758.

Jung H-J, Lee JM, Yang SH, Young SG, Fong LG, 2013. Nuclear lamins in the brain: new insights into function and regulation. Molecular Neurobiology 47, 290-301. 
Kadener S, Menet JS, Sugino K, Horwich MD, Weissbein U, Nawathean P, Vagin VV, Zamore PD, Nelson SB, Rosbash M, 2009. A role for microRNAs in the Drosophila circadian clock. Genes \& Development 23, 2179-2191.

Kaiser M, Libersat F, 2015. The role of the cerebral ganglia in the venom-induced behavioral manipulation of cockroaches stung by the parasitoid jewel wasp. Journal of Experimental Biology 218, 1022-1027.

Kamita SG, Nagasaka K, Chua JW, Shimada T, Mita K, Kobayashi M, Maeda S, Hammock BD, 2005. A baculovirus-encoded protein tyrosine phosphatase gene induces enhanced locomotory activity in a lepidopteran host. Proceedings of the National Academy of Sciences of the United States of America 102, 2584-2589.

Katsuma S, 2015. Baculovirus controls host catapillars by manipulating host physiology and behavior. Agri-Bioscience Monographs 5, 1-27.

Katsuma S, Koyano Y, Kang W, Kokusho R, Kamita SG, Shimada T, 2012. The baculovirus uses a captured host phosphatase to induce enhanced locomotory activity in host caterpillars. PLoS Pathogens 8, e1002644.

Katsuma S, Mita K, Shimada T, 2007. ERK- and JNK-dependent signaling pathways contribute to Bombyx mori nucleopolyhedrovirus infection. Journal of Virology 81, 13700-13709.

Katsuma S, Shimada T, 2015. The killing speed of egt-inactivated Bombyx mori nucleopolyhedrovirus depends on the developmental stage of B. mori larvae. Journal of Invertebrate Pathology 126, 64-70.

Ke J, Wang J, Deng R, Wang X, 2008. Autographa californica multiple nucleopolyhedrovirus ac66 is required for the efficient egress of nucleocapsids from the nucleus, general synthesis of preoccluded virions and occlusion body formation. Virology 374, 421-431.

Keene AC and Sprecher SG, 2012. Seeing the light: photobehavior in fruit fly larvae. Trends in Neurosciences 35, 104-110.

Kim YH, Lee DH, Jeong JH, Guo ZS, Lee YJ, 2008. Quercetin augments TRAIL-induced apoptotic death: involvement of the ERK signal transduction pathway. Biochemistry Pharmacology 75, 1946-1958.

Kleespies RG, Tidona CA, Darai G, 1999. Characterization of a new iridovirus isolated from crickets and investigations on the host range. Journal of Invertebrate Pathology 73, 84-90.

Knebel-Morsdorf D, Flipsen JT, Roncarati R, Jahnel F, Kleefsman AW, Vlak JM, 1996. Baculovirus infection of Spodoptera exigua larvae: lacZ expression driven by promoters of early genes pe38 and me53 in larval tissue. Journal of General Virology 77, 815-824.

Kool M, Ahrens CH, Vlak JM, Rohrmann GF, 1995. Replication of baculovirus DNA. Journal of General Virology 76, 21032118 .

Kulinčević JM, Stairs GR, Rothenbuhler WC, 1969. A disease of the honey bee causing behavioral changes and mortality. Journal of Invertebrate Pathology 14, 13-17.

Kvansakul M, 2017. Viral infection and apoptosis. Viruses 9, 356.

Lacey LA, Grzywacz D, Shapiro-Ilan DI, Frutos R, Brownbridge M, Goettel MS, 2015. Insect pathogens as biological control agents: Back to the future. Journal of Invertebrate Pathology 132, 1-41.

Lafferty KD, Kruis AM, 2012. Ecological consequences of manipulative parasites. In Hughes DP, Brodeur J, Thomas F (eds) Host manipulation by parasites. Oxford University Press, Oxford, 158-171.

Lafferty KD, Shaw JC, 2013. Comparing mechanisms of host manipulation across host and parasite taxa. Journal of Experimental Biology 216, 56-66.

Lai Q, Wu W, Li A, Wang W, Yuan M, Yang K, 2018. The 38K-mediated specific dephosphorylation of the viral core protein P6.9 plays an important role in the nucleocapsid assembly of Autographa californica multiple nucleopolyhedrovirus. Journal of Virology 92:e01989-17.

Langer T, Lu C, Echols H, Flanagan J, Hayer MK, Hartl FU, 1992. Successive action of DnaK, DnaJ and GroEL along the pathway of chaperone-mediated protein folding. Nature 356, 683-689.

Lawniczak MKN, Barnes AI, Linklater JR, Boone JM, Wigby S, Chapman T, 2007. Mating and immunity in invertebrates. Trends in Ecology \& Evolution 22, 48-55. 
Lee JH, Rowley WA, Platt KB, 2000. Longevity and spontaneous flight activity of Culex tarsalis (Diptera: Culicidae) infected with western equine encephalomyelitis virus. Journal of Medical Entomology 37, 187-193.

Lefevre T, Adamo SA, Biron DG, Misse D, Hughes D, Thomas F, 2009. invasion of the body snatchers: the diversity and evolution of manipulative strategies in host-parasite interactions. Advances in Parasitology 68, 45-83.

Lefèvre T, Thomas F, 2008. Behind the scene, something else is pulling the strings: emphasizing parasitic manipulation in vector-borne diseases. Infection, Genetics and Evolution 8, 504-519.

Lehiy CJ, Wu W, Berretta MF, Passarelli AL, 2013. Autographa californica M nucleopolyhedrovirus open reading frame 109 affects infectious budded virus production and nucleocapsid envelopment in the nucleus of cells. Virology 435, 442452.

Leisy DJ, Rasmussen C, Kim HT, Rohrmann GF, 1995. The Autographa californica nuclear polyhedrosis virus homologous region 1a: identical sequences are essential for DNA replication activity and transcriptional enhancer function. Virology 208:742-752.

Leman JC, Weddle CB, Gershman SN, Kerr AM, Ower GD, St John JM, Vogel LA, Sakaluk SK, 2009. Lovesick: immunological costs of mating to male sagebrush crickets. Journal of Evolution Biology 22, 163-171.

Li K, Wang Y, Bai H, Wang Q, Song J, Zhou Y, Wu C, Chen X, 2010. The putative pocket protein binding site of Autographa californica nucleopolyhedrovirus BV/ODV-C42 is required for virus-induced nuclear actin polymerization. Journal of Virology 84, 7857-7868.

Li SC, Shiau CK, Lin WC, 2007. Vir-Mir db: prediction of viral microRNA candidate hairpins. Nucleic Acids Research 36. 184-189.

Li SJ, Hopkins RJ, Zhao YP, Zhang YX, Hu J, Chen XY, Xu Z, Huang GH, 2016. Imperfection works: Survival, transmission and persistence in the system of Heliothis virescens ascovirus 3h (HvAV-3h), Microplitis similis and Spodoptera exigua. Scientific Reports 6, 21296.

Li Y, Guarino LA, 2008. Roles of LEF-4 and PTP/BVP RNA triphosphatases in processing of baculovirus late mRNAs. Journal of Virology 82, 5573-5583.

Li Y, Miller LK, 1995. Properties of a baculovirus mutant defective in the protein phosphatase gene. Journal of Virology 69 , 4533-4537.

Li Z, Chen Y, Zhang S, Chen S, Li W, Yan L, Shi L, Wu L, Sohr A, Su S, 2013. Viral infection affects sucrose responsiveness and moming ability of forager honey bees, Apis mellifera L. PLoS One 8, e77354.

Libersat F, Delago A, Gal R, 2009. Manipulation of host behaviour by parasitic insects and insect parasites. Annual Review of Entomology 54, 189-207.

Lima-Camara TN, Bruno RV, Luz PM, Castro MG, Lourenco-de-Oliveira R, Sorgine MH, Peixoto AA, 2011. Dengue infection increases the locomotor activity of Aedes aegyptifemales. PLoS One 6, 0017690.

Lindström MS, Nistér M, 2010. Silencing of ribosomal protein S9 elicits a multitude of cellular responses inhibiting the growth of cancer cells subsequent to p53 activation. PLoS One 5:e9578.

Long SR, Staskawicz BJ, 1993. Prokaryotic plant parasites. Cell 73, 921-935.

Lu A, Miller LK, 1995. The roles of eighteen baculovirus late expression factor genes in transcription and DNA replication Journal of Virology 69, 975-982.

Lucarotti C J, Whittome-Waygood B H, Lapointe R, et al., 2012. Pathology of a Gammabaculovirus in its natural balsam fir sawfly (Neodiprion abietis) host. Psyche: A Journal of Entomology 2012, 13.

Luckow VA, Lee SC, Barry GF, Olins PO, 1993. Efficient generation of infectious recombinant baculoviruses by site-specific transposon-mediated insertion of foreign genes into a baculovirus genome propagated in Escherichia coli. Journal of Virology 67, 4566-4579.

Lundell MJ, Hirsh J, 1994. Temporal and spatial development of serotonin and dopamine neurons in the Drosophila CNS. Developmental Biology 165, 385-396. 
Maizels RM, McSorley HJ, 2016. Regulation of the host immune system by helminth parasites. The Journal of Allergy and Clinical Immunology 138, 666-675.

Marek M, Merten OW, Francis-Devaraj F, Oers MM, 2012. Essential C-terminal region of the baculovirus minor capsid protein VP80 binds DNA. Journal of Virology 86, 1728-1738.

Marek M, Merten O-W, Galibert L, Vlak JM, van Oers MM, 2011. Baculovirus VP80 protein and the F-actin cytoskeleton interact and connect the viral replication factory with the nuclear periphery. Journal of Virology 85, 5350-5362.

Marek M, Romier C, Galibert L, Merten O-W, van Oers MM, 2013. Baculovirus VP1054 is an acquired cellular PUR $\alpha$, a nucleic acid-binding protein specific for GGN repeats. Journal of Virology 87, 8465-8480.

Martinez J, Duplouy A, Woolfit M, Vavre F, O’Neill SL, Varaldi J, 2012. Influence of the virus LbFV and of Wolbachia in a host-parasitoid interaction. PLoS One 7, e35081.

Mauck K, Bosque-Pérez NA, Eigenbrode SD, de Moraes CM, Mescher MC, 2012. Transmission mechanisms shape pathogen effects on host-vector interactions: evidence from plant viruses. Functional Ecology 26, 1162-1175.

Maure F, Daoust SP, Brodeur J, Mitta G, Thomas F, 2013. Diversity and evolution of bodyguard manipulation. Journal of Experimental Biology 216, 36-42.

Maynard BJ, DeMartini L, Wright WG, 1996. Gammarus lacustris harboring Polymorphus paradoxus show altered patterns of serotonin-like immunoreactivity. Journal of Parasitology 82, 663-666.

McCarthy CB, Dai X, Donly C, Theilmann DA, 2008. Autographa californica multiple nucleopolyhedrovirus ac142, a core gene that is essential for BV production and ODV envelopment. Virology 372, 325-339.

McGinty RK, Kim J, Chatterjee C, Roeder RG, Muir TW, 2008. Chemically ubiquitylated histone H2B stimulates hDot1Lmediated intranucleosomal methylation. Nature 453, 812-816.

Medina-Ortega KJ, Bosque-Perez NA, Ngumbi E, Jimenez-Martinez ES, Eigenbrode SD, 2009. Rhopalosiphum padi (Hemiptera: Aphididae) responses to volatile cues from Barley yellow dwarf virus-infected wheat. Environmental Entomology 38, 836-845.

Mehlhorn H, 2015. Parasites: an own world of cross reactions with their hosts. In Mehlhorn H, editor. Host Manipulations by Parasites and Viruses Cham: Springer International Publishing. p. 3-27.

Mehrkhou F, Talebi AA, Moharramipour S, Naveh VH, 2012. Demographic parameters of Spodoptera exigua (Lepidoptera: Noctuidae) on different soybean cultivars. Environmental Entomology 41, 326-332.

Miller LK, Ball LA, 1998. The insect viruses. Plenum Press, New York.

Moreno-Delafuente A, Garzo E, Moreno A, Fereres A, 2013. A plant virus manipulates the behavior of its whitefly vector to enhance its transmission efficiency and spread. PLoS One 8, e61543.

Moscardi F, 1999. Assessment of the application of baculoviruses for control of Lepidoptera. Annual Review of Entomology 44, 257-289.

Mu J, van Lent JW, Smagghe G, Wang Y, Chen X, Vlak JM, van Oers MM, 2014. Live imaging of baculovirus infection of midgut epithelium cells: a functional assay of per os infectivity factors. Journal of General Virology 95, 2531-2539.

Murillo R, Elvira S, Munoz D, Williams T, Caballero P, 2006. Genetic and phenotypic variability in Spodoptera exigua nucleopolyhedrovirus isolates from greenhouse soils in southern Spain. Biological Control 38, 157-165.

Nagamine T, Kawasaki Y, lizuka T, Matsumoto S, 2005. Focal distribution of baculovirus IE1 triggered by its binding to the hr DNA elements. Journal of Virology 79, 39-46.

Nalçacıoğlu R, Ince I, Demirbağ Z, 2009. The biology of Chilo iridescent virus. Virology Sinica 24, 285-294.

Nguyen Q, Nielsen LK, Reid S, 2013. Genome scale transcriptomics of baculovirus-insect interactions. Viruses 5, $2721-2747$.

Ni S, Yan Y, Cui H, Yu Y, Huang Y, Qin Q, 2017. Fish miR-146a promotes Singapore grouper iridovirus infection by regulating cell apoptosis and NF-kappaB activation. The Journal of General Virology 98, 1489-1499.

Ohkawara K, Aonuma H, 2016. Changes in the levels of biogenic amines associated with aggressive behavior of queen in the social parasite ant Vollenhovia nipponica. Insectes Sociaux 63, 257-264.

Online Etymology Dictionary. https://www.etymonline.com/, 2017. 
Ono C, Kamagata T, Taka H, Sahara K, Asano S, Bando H, 2012. Phenotypic grouping of 141 BmNPVs lacking viral gene sequences. Virus Research 165, 197-206.

O'Reilly DR, 1995. Baculovirus-encoded ecdysteroid UDP-glucosyltransferases. Insect Biochemistry and Molecular Biology $25,541-550$.

O'Reilly DR, Miller LK, 1989. A baculovirus blocks insect molting by producing ecdysteroid UDP-glucosyl transferase. Science 245, 1110-1112.

O'Reilly DR, Miller LK, 1991. Improvement of a baculovirus pesticide by deletion of the egt gene. Nature Biotechnology 9 , 1086.

Otsuna H, Shinomiya K, Ito K, 2014. Parallel neural pathways in higher visual centers of the Drosophila brain that mediate wavelength-specific behavior. Frontiers in Neural Circuits 8, 1-8.

Passarelli AL, 2011. Barriers to success: How baculoviruses establish efficient systemic infections. Virology 411, $383-392$.

Patot S, Lepetit D, Charif D, Varaldi J, Fleury F, 2009. Molecular detection, penetrance, and transmission of an inherited virus responsible for behavioral manipulation of an insect parasitoid. Applied Environmental Microbiology 75, 703-710.

Patot S, Martinez J, Allemand R, Gandon S, Varaldi J, Fleury F, 2010. Prevalence of a virus inducing behavioural manipulation near species range border. Molecular Ecology 19, 2995-3007.

Patterson KI, Brummer T, O'Brien PM, Daly RJ, 2009. Dual-specificity phosphatases: critical regulators with diverse cellular targets. Journal of Biochemistry 418, 475-489.

Peng K, van Oers MM, Hu Z, van Lent JW, Vlak JM, 2010. Baculovirus per os infectivity factors form a complex on the surface of occlusion-derived virus. Journal of Virology 84, 9497-9504.

Perez-Chacon G, de Los Rios C, Zapata JM, 2014. Indole-3-carbinol induces cMYC and IAP-family downmodulation and promotes apoptosis of Epstein-Barr virus (EBV)-positive but not of EBV-negative Burkitt's lymphoma cell lines. Pharmacological Research 89, 46-56.

Perrot-Minnot MJ, Cezilly F, 2013. Investigating candidate neuromodulatory systems underlying parasitic manipulation: concepts, limitations and prospects. Journal of Experimental Biology 216, 134-141.

Pijlman GP, Dortmans JC, Vermeesch AM, Yang K, Martens DE, Goldbach RW, Vlak JM, 2002. Pivotal role of the non-hr origin of DNA replication in the genesis of defective interfering baculoviruses. Journal of Virology 76, 5605-5611.

Platt K, Linthicum K, Myint K, Innis B, Lerdthusnee K, Vaughn D, 1997. Impact of dengue virus infection on feeding behavior of Aedes aegypti. The American Journal of Tropical Medicine and Hygiene 57, 119-125.

Ponton F, Lefèvre T, Lebarbenchon C, Thomas F, Loxdale HD, Marché L, Renault L, Perrot-Minnot MJ, Biron DG, 2006. Do distantly related parasites rely on the same proximate factors to alter the behavior of their hosts? Proceedings of the Royal Society of London B: Biological Sciences 273, 2869-2877.

Ponton F, Otálora-Luna F, Lefèvre T, Guerin PM, Lebarbenchon C, Duneau D, Biron DG, Thomas F, 2011. Water-seeking behavior in worm-infected crickets and reversibility of parasitic manipulation. Behaviour Ecology 22, 392-400.

Porras A, Zuluaga S, Black E, Valladares A, Alvarez AM, Ambrosino C, Benito M, Nebreda AR, 2004. P38 alpha mitogenactivated protein kinase sensitizes cells to apoptosis induced by different stimuli. Molecular Biology of the Cell 15, 922-933.

Poulin R, 2000. Manipulation of host behaviour by parasites: a weakening paradigm? Proceedings of the Royal Society of London Series B 267.

Prikhod'ko EA, Miller LK, 1996. Induction of apoptosis by baculovirus transactivator IE1. Journal of Virology 70, 7116-7124.

Pruijssers AJ, Strand MR, 2007. PTP-H2 and PTP-H3 from Microplitis demolitor bracovirus localize to focal adhesions and are antiphagocytic in insect immune cells. Journal of Virology 81, 1209-1219.

Pullen SS, Friesen PD, 1995. Early transcription of the ie- 1 transregulator gene of Autographa californica nuclear polyhedrosis virus is regulated by DNA sequences within its 5' noncoding leader region. Journal of Virology 69, 156-165.

Putnam JL, Scott TW, 1995. Blood-feeding behavior of dengue-2 virus-infected Aedes aegypti. The American Journal of Tropical Medicine and Hygiene 52, 225-227. 
Qualls WA, Day JF, Xue RD, Bowers DF, 2012. Sindbis virus infection alters blood feeding responses and DEET repellency in Aedes aegypti (Diptera: Culicidae). Journal of Medical Entomology 49, 418-423.

R: A language and environment for statistical computing. Version 3.0.0. 2013.Vienna, Austria: R Foundation for Statistical Computing.

Raina AK, Adams JR, 1995. Gonad-specific virus of corn earworm. Nature 374, 770.

Raina AK, Kingan TG, Giebltowicz JM, 1994. Mating-induced loss of sex pheromone and sexual receptivity in insects with emphasis on Helicoverpa zea and Lymantria dispar. Archives of insect biochemistry and physiology 25, 317-327.

Rajabaskar D, Bosque-Pérez NA, Eigenbrode SD, 2014. Preference by a virus vector for infected plants is reversed after virus acquisition. Virus Research 186, 32-37.

Raymond B, Hartley SE, Cory JS, Hails RS, 2005. The role of food plant and pathogen-induced behaviour in the persistence of a nucleopolyhedrovirus. Journal of Invertebrate Pathology 88, 49-57.

Redman KL, Rechsteiner M, 1989. Identification of the long ubiquitin extension as ribosomal protein S27a. Nature 338, 438440.

Reese SM, Beaty MK, Gabitzsch ES, Blair CD, Beaty BJ, 2009. Aedes triseriatus females transovarially infected with La Crosse virus mate more efficiently than uninfected mosquitoes. Journal of Medical Entomology 46, 1152-1158.

Reichert H, Boyan G, 1997. Building a brain: developmental insights in insects. Trends in Neurosciences 20, 258-264.

Reilly LM, Guarino LA, 1994. The pk-1 gene of Autographa californica multinucleocapsid nuclear polyhedrosis virus encodes a protein kinase. Journal of General Virology 75, 2999-3006.

Richard FJ, Aubert A, Grozinger CM, 2008. Modulation of social interactions by immune stimulation in honey bee, Apis mellifera, workers. BMC Biology 6, 50.

Richard FJ, Holt HL, Grozinger CM, 2012. Effects of immunostimulation on social behaviour, chemical communication and genome-wide gene expression in honey bee workers (Apis mellifera). BMC Genomics 13, 558.

Robertson J, Preisler H, Russell R, 2007. PoloPlus: Probit and logit analysis user's guide. LeOra Software, Petaluna, CA, USA.

Rodriguez-Moncalvo VG, Campos AR, 2009. Role of serotonergic neurons in the Drosophila larval response to light. BMC Neuroscience 10, 66.

Rohrmann GF, 2013. The baculovirus replication cycle: effects on cells and insects. In: Baculovirus Molecular Biology. National Center for Biotechnology, Bethesda, MD, USA.

Rohrmann GF, 2013b. Structural proteins of baculovirus occlusion bodies and virions. In: Baculovirus Molecular Biology. National Center for Biotechnology, Bethesda, MD, USA.

Rortais A, Tentcheva D, Papachristoforou A, Gauthier L, Arnold G, Colin ME, Bergoin M, 2006. Deformed wing virus is not related to honey bees' aggressiveness. Journal of Virology 3, 61.

Ros VID, van Houte S, Hemerik L, van Oers MM, 2015. Baculovirus-induced tree-top disease: how extended is the role of egt as a gene for the extended phenotype? Molecular Ecology 24,249-258.

Rosenberg LA, Glusman JG, Libersat F, 2007. Octopamine partially restores walking in hypokinetic cockroaches stung by the parasitoid wasp Ampulex compressa. Journal of Experimental Biology 210, 4411-4417.

Salvy M, Martin C, Bagnères AG, Provost É, Roux M, Le Conte Y, Clément JL, 2001. Modifications of the cuticular hydrocarbon profile of Apis mellifera worker bees in the presence of the ectoparasitic mite Varroa jacobsoni in brood cells. Parasitology 122, 145-159.

Sato T, Watanabe K, Kanaiwa M, Niizuma Y, Harada Y, Lafferty KD, 2011. Nematomorph parasites drive energy flow through a riparian ecosystem. Ecology 92, 201-207.

Schöning C, Gisder S, Geiselhardt S, Kretschmann I, Bienefeld K, Hilker M, Genersch E, 2012. Evidence for damagedependent hygienic behaviour towards Varroa destructor-parasitised brood in the western honey bee, Apis mellifera. Journal of Experimental Biology 215, 264-271.

Schoofs A, Huckesfeld S, Surendran S, Pankratz MJ, 2014. Serotonergic pathways in the Drosophila larval enteric nervous system. Journal of Insect Physiology 69, 118-125. 
Schultz KLW, Friesen PD, 2009. Baculovirus DNA replication-specific expression factors trigger apoptosis and shutoff of host protein synthesis during infection. Journal of Virology 83, 11123-11132.

Schultz KLW, Wetter JA, Fiore DC, Friesen PD, 2009. Transactivator IE1 is required for baculovirus early replication events that trigger apoptosis in permissive and nonpermissive cells. Journal of Virology 83, 262-272.

Schultz-Cherry S, Dybdahl-Sissoko N, Neumann G, Kawaoka Y, Hinshaw VS, 2001. Influenza virus NS1 protein induces apoptosis in cultured cells. Journal of Virology 75, 7875-7881.

Schulze SR, Curio-Penny B, Speese S, Dialynas G, Cryderman DE, McDonough CW, Nalbant D, Petersen M, Budnik V, Geyer PK, Wallrath LL, 2009. A comparative study of Drosophila and human A-type lamins. PLOS One 4, e7564.

Shah KS, Evans EC, Pizzorno MC, 2009. Localization of deformed wing virus (DWV) in the brains of the honeybee, Apis mellifera Linnaeus. Journal of Virology 6, 182.

Sheng Z, Charbonneau H, 1993. The baculovirus Autographa californica encodes a protein tyrosine phosphatase. Journal of Biological Chemistry 268, 4728-4733.

Shevchenko A, Tomas H, Havlis J, Olsen JV, Mann M, 2006. In-gel digestion for mass spectrometric characterization of proteins and proteomes. Nature Protocols 1, 2856-2860.

Simón O, Williams T, Asensio AC, Ros S, Gaya A, Caballero P, Possee RD, 2008. Sf29 gene of Spodoptera frugiperda multiple nucleopolyhedrovirus is a viral factor that determines the number of virions in occlusion bodies. Journal of Virology $82,7897-7904$.

Slack J, Arif BM, 2007. The baculoviruses occlusion-derived virus: virion structure and function. Advanced Virus Research 69, 99-165.

Smirnoff WA, 1965. Observations on the effect of virus infection on insect behavior. Journal of Invertebrate Pathology 7 , $387-388$.

Smits PH, Van Velden M, Van de Vrie M, Vlak JM, 1987. Feeding and dispersion of Spodoptera exigua larvae and its relevance for control with a nuclear polyhedrosis virus. Entomologia Experimentalis et Applicata 43, 67-72.

Smits PH, van De Vrie M, Vlak JM, 1986. Oviposition of beet armyworm (Lepidoptera: Noctuidae) on greenhouse crops. Environmental Entomology 15, 1189-1191.

Smits PH, Vlak JM, 1988. Biological activity of Spodoptera exigua nuclear polyhedrosis virus against S. exigua larvae. Journal of Invertebrate Pathology 51, 107-114.

Soares JS, Ribeiro BM, 2005. Pathology of Anticarsia gemmatalis larvae infected by two recombinant A. gemmatalis multicapsid nucleopolyhedroviruses. Research in Microbiology 156, 263-269.

Stafford CA, Walker GP, Ullman DE, 2011. Infection with a plant virus modifies vector feeding behavior. Proceedings of the National Academy of Sciences of the United States of America 108, 9350-9355.

Stapel JO, Waters DJ, Ruberson JR, Lewis WJ, 1998. Development and behavior of Spodoptera exigua (Lepidoptera: Noctuidae) Larvae in choice tests with food substrates containing toxins of Bacillus thuringiensis. Biological Control 11, 29-37.

Strauss R, 2002. The central complex and the genetic dissection of locomotor behaviour. Current Opinion in Neurobiology 12 , 633-638.

Suderman RJ, Pruijssers AJ, Strand MR, 2008. Protein tyrosine phosphatase-H2 from a polydnavirus induces apoptosis of insect cells. Journal of General Virology 89, 1411-1420.

Sumpter DJT, Martin SJ, 2004. The dynamics of virus epidemics in Varroa-infested honey bee colonies. Journal of Animal Ecology 73, 51-63.

Sun X, van der Werf W, Bianchi FJJA, Hu Z, Vlak JM, 2006. Modelling biological control with wild-type and genetically modified baculoviruses in the Helicoverpa armigera-cotton system. Ecological Modelling 198, 387-398.

Sun X-X, DeVine T, Challagundla KB, Dai M-S, 2011. Interplay between ribosomal protein S27a and MDM2 protein in p53 activation in response to ribosomal stress. Journal of Biological Chemistry 286, 22730-22741. 
Sun YX, Tian A, Zhang XB, Zhao ZG, Zhang ZW, Ma RY, 2014. Phototaxis of Grapholitha molesta (Lepidoptera: Olethreutidae) to different light sources. Journal of Economic Entomology 107, 1792-1799.

Suzuki N, Nonaka H, Tsuge Y, Inui M, Yukawa H, 2005. New multiple-deletion method for the Corynebacterium glutamicum genome, using a mutant lox sequence. Applied and Environmental Microbiology 71, 8472-8480.

Tain L, Perrot-Minnot MJ, and Cézille F, 2006. Altered host behavior and brain serotonergic activity caused by acanthocephalans: evidence for specificity. Proceedings of the Royal Society of London B: Biological Sciences 273, 3039-3045.

Tain L, Perrot-Minnot M-J, Cézilly F, 2006. Altered host behaviour and brain serotonergic activity caused by acanthocephalans: evidence for specificity. Proceedings of the Royal Society B: Biological Sciences 273, 3039-3045.

Takagi T, Taylor GS, Kusakabe T, Charbonneau H, Buratowski S, 1998. A protein tyrosine phosphatase-like protein from baculovirus has RNA 5'-triphosphatase and diphosphatase activities. . Proceedings of the National Academy of Sciences of the United States of America 95:9808-9812.

Thomas F, Adamo S, Moore J, 2005. Parasitic manipulation: where are we and where should we go? Behaviour Processes 68, 185-199.

Thomas F, Schmidt-Rhaesa A, Martin G, Manu C, Durand P, Renaud F, 2002. Do hairworms (Nematomorpha) manipulate the water seeking behaviour of their terrestrial hosts? Journal of Evolution Biology 15,356-361.

Torquato EF, Neto M, Brancalhão R, 2006. Nucleopolyhedrovirus infected central nervous system cells of Bombyx mori (Lepidoptera: Bombycidae). Neotropical Entomology 35, 70-74.

Tully O, Nolan DT, 2002. A review of the population biology and host-parasite interactions of the sea louse Lepeophtheirus salmonis (Copepoda: Caligidae). Parasitology 124, 165-182.

Turell MJ, Gargan TP, Bailey CL, 1985. Culex pipiens (Diptera: Culicidae) morbidity and mortality associated with Rift Valley fever virus infection. Journal of Medical Entomology 22, 332-337.

Urabe M, Ding C, Kotin RM., 2002. Insect cells as a factory to produce adeno-associated virus type 2 vectors. Human Gene Therapy 13, 1935-1943.

van Alphen JJ, Visser ME, 1990. Superparasitism as an adaptive strategy for insect parasitoids. Annual Review of Entomology 351, 59-79.

van de Wetering F, Hulshof J, Posthuma K, Harrewijn P, Goldbach R, Peters D, 1998. Distinct feeding behavior between sexes of Frankliniella occidentalis results in higher scar production and lower tospovirus transmission by females. Entomologia Experimentalis et Applicata. 88, 9-15.

van Houte S, Ros VID, Mastenbroek TG, Vendrig NJ, Hoover K, Spitzen J, van Oers MM, 2012. Protein tyrosine phosphataseinduced hyperactivity is a conserved strategy of a subset of baculoviruses to manipulate lepidopteran host behavior. PLoS One 7:e46933.

van Houte S, Ros VID, van Oers MM, 2013. Walking with insects: molecular mechanisms behind parasitic manipulation of host behaviour. Molecular Ecology 22, 3458-3475.

van Houte S, Ros VID, van Oers MM, 2014a. Hyperactivity and tree-top disease induced by the baculovirus AcMNPV in Spodoptera exigua larvae are governed by independent mechanisms. Naturwissenschaften 101, 347-350.

van Houte S, van Oers MM, Han Y, Vlak JM, Ros VID, 2014b. Baculovirus infection triggers a positive phototactic response in caterpillars to induce 'tree-top' disease. Biology Letters 10, 20140680.

van Houte S, van Oers MM, Han Y, Vlak JM, Ros VID, 2015. Baculovirus infection triggers a positive phototactic response in caterpillars: a response to Dobson et al. (2015). Biology Letters 11, 20150633.

van Oers MM, 2010. Genomics and biology of Iflaviruses. Insect Virology Caister Academic Press, Norfolk, 231-250.

van Oers MM, Pijlman GP, Vlak JM, 2015. Thirty years of baculovirus-insect cell protein expression: from dark horse to mainstream technology. Journal of General Virology 96, 6-23.

van Oers MM, van Marwijk M, Kwa MS, Vlak JM, Thomas AA, 1999. Cloning and analysis of cDNAs encoding the hypusinecontaining protein eIF5A of two lepidopteran insect species. Insect Molecular Biology 8, 531-538. 
van Oers MM, Vlak JM, 2007. Baculovirus genomics. Current Drug Targets 8, 1051-1068.

Vanarsdall AL, Pearson MN, Rohrmann GF, 2007. Characterization of baculovirus constructs lacking either the Ac 101, Ac 142, or the Ac 144 open reading frame. Virology 367, 187-195.

Vanfleteren JR, Van Bun SM, Delcambe LL, Van Beeumen JJ, 1986. Multiple forms of histone H2B from the nematode Caenorhabditis elegans. Biochemical Journal 235, 769-773.

Varaldi J, Bouletreau M, Fleury F, 2005. Cost induced by viral particles manipulating superparasitism behaviour in the parasitoid Leptopilina boulardi. Parasitology 131, 161-168.

Varaldi J, Fouillet P, Ravallec M, Lopez-Ferber M, Bouletreau M, Fleury F, 2003. Infectious behaviour in a parasitoid. Science 302, 1930.

Varaldi J, Martinez J, Patot S, Lepetit D, Fleury F, Gandon S, Drezen JM, Beckage NE, 2012/ An inherited virus manipulating the behaviour of its parasitoid host: epidemiology and evolutionary consequences. In: Beckage NE, Drezen JM (eds) Parasitoid viruses: symbionts and pathogens. Academic, San Diego, 203-214.

Varaldi J, Patot S, Nardin M, Gandon S, 2009. A virus-shaping reproductive strategy in a Drosophila parasitoid. Advanced Parasitology 70, 333-363.

Varaldi J, Petit S, Bouletreau M, Fleury F, 2006. The virus infecting the parasitoid Leptopilina boulardi exerts a specific action on superparasitism behaviour. Parasitology 132, 747-756.

Vasconcelos SD, 1996. Alternative routes for the horizontal transmission of a nucleopolyhedrovirus. Journal of Invertebrate Pathology 68, 269-274.

Vasconcelos SD, Cory JS, Wilson KR, Sait SM, Hails RS, 1996. Modified behaviour in baculovirus-infected lepidopteran larvae and its impact on the spatial distribution of inoculum. Biological Control 7, 299-306.

Vialard JE, Richardson CD, 1993. The 1,629-nucleotide open reading frame located downstream of the Autographa californica nuclear polyhedrosis virus polyhedrin gene encodes a nucleocapsid-associated phosphoprotein. Journal of Virology $67,5859-5866$.

Vijay N, Poelstra JW, Künstner A, Wolf JB, 2013. Challenges and strategies in transcriptome assembly and differential gene expression quantification. A comprehensive in silico assessment of RNA-seq experiments. Molecular Ecology 22, 620-634.

Volkman LE, 1988. Autographa californica MNPV nucleocapsid assembly: inhibition by cytochalasin D. Virology 163, 547553.

Volkman LE, 2007. Baculovirus infectivity and the actin cytoskeleton. Current Drug Targets 8, 1075-1083.

Wan N-F, Ji X-Y, Zhang H, Yang J-H, Jiang J-X, 2015. Nucleopolyhedrovirus infection and/or parasitism by Microplitis pallidipes Szepligeti affect hemocyte apoptosis of Spodoptera exigua (Hübner) larvae. Journal of Invertebrate Pathology 132, 165-170.

Wang G, Zhang J, Shen Y, Zheng Q, Feng M, Xiang X, Wu X, 2015. Transcriptome analysis of the brain of the silkworm Bombyx mori infected with Bombyx mori nucleopolyhedrovirus: A new insight into the molecular mechanism of enhanced locomotor activity induced by viral infection. Journal of Invertebrate Pathology 128, 37-43.

Wang L, Yu J, Yin C, Li Z, Hu X, Pang Y, 2002. Characterization of a J domain gene of Spodoptera litura multicapsid nucleopolyhedrovirus. Virus Genes 25, 291-297.

Wang R, Deng F, Hou D, Zhao Y, Guo L, Wang H, Hu Z, 2010. Proteomics of the Autographa californica nucleopolyhedrovirus budded virions. Journal of Virology 84, 7233-7242.

Wang Y, Wu W, Li Z, Yuan M, Feng G, Yu Q, Yang K, Pang Y, 2007. Ac18 is not essential for the propagation of Autographa californica multiple nucleopolyhedrovirus. Virology 367, 71-81.

Wesołowska W, Wesołowski T, 2014. Do Leucochloridium sporocysts manipulate the behavior of their snail hosts? Journal of Zoology 292, 151-155. 
Whitford M, Stewart S, Kuzio J, Faulkner P, 1989. Identification and sequence analysis of a gene encoding GP67, an abundant envelope glycoprotein of the baculovirus Autographa californica nuclear polyhedrosis virus. Journal of Virology 63 , 1393-1399.

Williams T, Bergoin M, van Oers MM, 2017. Diversity of large DNA viruses of invertebrates. Journal of Invertebrate Pathology 147, 4-22.

Williamson C, Rybicki EP, Kasdorf GGF, Von Wechmar MB, 1988. Characterization of a new picorna-like virus isolated from aphids. Journal of General Virology 69, 787-795.

Wilson-Rich N, Spivak M, Fefferman NH, Starks PT, 2009. Genetic, individual, and group facilitation of disease resistance in insect societies. Annual Review of Entomology 54, 405-423.

Wu C, Deng Z, Long Z, Cai Y, Ying Z, Yin H, Yuan M, Clem RJ, Yang K, Pang Y, 2016. Generating a host range-expanded recombinant baculovirus. Scientific Reports 6, 28072.

Wu W, Liang H, Kan J, Liu C, Yuan M, Liang C, Yang K, Pang Y, 2008. Autographa californica multiple nucleopolyhedrovirus $38 \mathrm{~K}$ is a novel nucleocapsid protein that interacts with VP1054, VP39, VP80, and itself. Journal of Virology 82, 12356-12364.

Xeros N, 1956. The virogenic stroma in nuclear and cytoplasmic polyhedroses. Nature 178, 412.

Xu F, Ince IA, Boeren S, Vlak JM, van Oers MM, 2011. Protein composition of the occlusion derived virus of Chrysodeixis chalcites nucleopolyhedrovirus. Virus Research 158, 1-7.

Yamaguchi S, Heisenberg M, 2011. Photoreceptors and neural circuitry underlying phototaxis in insects. Fly 5, 333-336.

Yang M, Wang S, Yue XL, Li LL, 2014. Autographa californica multiple nucleopolyhedrovirus orf132 encodes a nucleocapsid-associated protein required for budded-virus and multiply enveloped occlusion-derived virus production. Journal of Virology 88, 12586-12598.

Yang X, Cox-Foster DL, 2005. Impact of an ectoparasite on the immunity and pathology of an invertebrate: evidence for host immunosuppression and viral amplification. Proceedings of the National Academy of Sciences of the United States of America 102, 7470-7475.

Young SG, Jung H-J, Coffinier C, Fong LG, 2012. Understanding the roles of nuclear A- and B-type Lamins in brain development. Journal of Biological Chemistry 287, 16103-16110.

Zanotto PM, Kessing BD, Maruniak JE, 1993. Phylogenetic interrelationships among baculoviruses: evolutionary rates and host associations. Journal of Invertebrate Pathology 62, 147-164.

Zhang X, Xu K, Wei D, Wu W, Yang K, Yuan M, 2017. Baculovirus infection induces disruption of the nuclear lamina. Scientific Reports 7, 7823.

Zhu L-N, Mei C-L, Zhang Z-X, 2008. The function of SeMNPV IAP3 in mammalian cells. Virology Sinica 23, 183-188. 


\section{Summary}

An infection by parasites affects many aspects of the invaded hosts, such as morphology, physiology, development and behaviour. While some of these changes are adaptive for the host, some of these changes appear to be adaptive to the parasites. Host behavioural manipulation has been reported for many parasite-host combinations. An intriguing example and the main topic of study in this thesis, is host behavioural manipulation by baculoviruses, in which host insects serve as a tool to enhance the transmission rate, and hence fitness, of these viruses.

Chapter 2 of this thesis reviews the documented changes of insect behaviour upon virus infection and summarizes the limited knowledge available so far about the underlying mechanisms. Special attention is given to baculovirus-induced behavioural changes in caterpillars. Extensive information on baculovirus genomics is available and the role of several viral genes in this process has been studied. The ecological and evolutionary consequences of virus-induced insect behavioural changes are also discussed.

Baculoviruses induce pre-death climbing behaviour in their lepidopteran hosts. The phenomenon is also known as "tree-top disease" or "Wipfelkrankheit". Death at elevated positions is thought to disperse progeny virus over larger areas of plant foliage. Hoover et al. (Science, 2011) found that the egt gene from the baculovirus Lymantria dispar multiple nucleopolyhedrovirus (LdMNPV) was involved in inducing tree-top disease in L. dispar larvae. Our previous work revealed that Spodoptera exigua (Se)MNPV induced tree-top disease in $S$. exigua larvae, which is light-dependent. Chapter 3 aimed to determine whether the egt gene from SeMNPV is also involved in SeMNPV-induced tree-top disease. The results in Chapter 3 showed that $S$. exigua larvae infected with SeMNPV lacking the egt gene died at low positions. The EGT enzyme is known to inactivate the insect moulting hormone and often has an impact on the time to death of the infected host. Spodoptera exigua larvae infected with a mutant virus lacking the egt gene died earlier than larvae infected with wild type (WT) virus. Our data show that larvae infected with the mutant virus died before the onset of the climbing behaviour seen in wild-type infected larvae. Therefore, we concluded that SeMNPV EGT enzyme facilitates tree-top disease in infected S. exigua larvae by extending the survival time of its host.

Besides viral and host genes, environmental conditions may also affect the outcomes of behavioural manipulation. Light has been reported before to play an important function in SeMNPV-induced tree-top disease in S. exigua. In Chapter 4, the importance of the time point at which light is provided (relative to the moment of infection) in the induction of tree-top 
disease was investigated. The results showed that light from above was needed between 43 and 50 hours post infection (hpi) to induce tree-top disease. This time interval is prior to the actual climbing, which occurred in the dark between 57 and 67 hpi. When infected larvae were not exposed to light between 43 and 50 hpi, they eventually died at low positions.

The second type of behavioural manipulation induced by baculoviruses in their hosts is enhanced locomotion behaviour (or hyperactivity). The induced hyperactivity contributes to virus spread over a larger area. Previous work demonstrated the involvement of the $p t p$ gene from Autographa californica (Ac)MNPV in triggering hyperactivity in S. exigua larvae. The phosphatase activity of the encoded PTP protein was also needed for this process, since mutating the catalytic site of PTP blocked the induction of hyperactivity in infected hosts. SeMNPV also carries a protein tyrosine phosphatase, called ptp2, which is phylogenetically unrelated to $p t p$. Ptp2 is more similar to the protein tyrosine phosphatase gene (ptph2) from Microplitis demolitor bracovirus, that induced apoptosis in host immune cells. The idea is that this induction of apoptosis reduces the number of immune cells and that as a consequence the virus can evade from immune responses, leading to increased virus propagation. In Chapter 5, we investigated whether the SeMNPV PTP2 protein also has a pro-apoptotic effect on cultured insect cells and on S. exigua immune cells. The results showed that PTP2 induced mild apoptosis in cultured insect cells (Sf21 cells) and that the catalytic site of PTP2 was needed for this process. Moreover, the SeMNPV ptp2 gene was also involved in inducing apoptosis in host hemocytes. Larvae infected with WT virus produced more viral occlusion bodies (OBs) than larvae infected with an SeMNPV mutant lacking the $p t p 2$ gene. We hypothesized that SeMNPV suppresses the host immune system by inducing apoptosis in hemocytes and that this immune suppression finally leads to higher OB yields.

Parasites may achieve behavioural manipulation by invading or affecting the host central nervous system (CNS), for instance by affecting biogenic amine levels in the host brain and we wondered whether that was also the case for baculoviruses. However, little information was available on the morphology and function of the CNS of S. exigua larvae, and of caterpillars in general. In Chapter 6, we first constructed a 3D model of the $S$. exigua larval brain and described different brain areas, by immunolabelling serotonergic cells in the larval brain and gnathal ganglion (GNG, or subesophageal ganglion,SEG). Furthermore, we described the distribution of serotonergic neuron cells in the brain and GNG. Individual cell clusters were mapped and the number of serotonergic cells in each cluster in the brain and the GNG were estimated. As a next step, we studied virus localization and temporal invasion patterns in the larval brain and 
GNG, using green fluorescent protein-labelled AcMNPV virus particles (GFP-AcMNPV). The results showed that AcMNPV started to invade the host CNS from the second day post infection (dpi) at the lateral sides, and further spread to specific cells throughout the brain at three dpi (when hyperactivity is observed). Electron microscopy results confirmed that AcMNPV replicates in the brain and GNG. The data in this chapter provided more information on the larval CNS and has pointed out several interesting directions for future research.

On the molecular level, AcMNPV PTP (AcPTP) must interact with a host or viral protein that leads to the induction of hyperactivity. To identify host and viral proteins that interact with AcPTP, a PTP substrate analysis was performed using a mass spectrometry approach. The results in Chapter 7 describe host and viral proteins that co-purified with AcPTP. The results indicate that AcPTP is potentially involved in many different steps of the virus replication cycle, including nucleocapsid assembly, nucleocapsid transport, ODV envelopment and/or virus dissemination. The results suggest that AcPTP functions both as a structural protein and as a phosphatase enzyme. Some of the interactions potentially have an important role in cellular signalling pathways and potentially in brain invasion.

Chapter 8 discusses the main findings of this thesis and gives implications regarding to future research on baculovirus-induced behavioural manipulation. Overall, the results of this thesis showed that the viral egt gene and the timing of light both play an important role in baculovirusinduced tree-top disease. Moreover, the $p t p 2$ gene plays a significant function in host immune system modulation. We studied the morphology of the S. exigua larval brain and the serotonergic neuron distribution in the host CNS and explored how baculoviruses invade the host CNS. Lastly, potential interaction partners of the PTP protein, involved in the induction of hyperactivity, were described. This work contributes to the fundamental knowledge on molecular mechanisms of parasitic manipulation of host behaviour. 


\section{List of publications}

1. Han Y , van Oers MM, van Houte S, Ros VID, 2015. Virus-induced behavioural changes in insects, in: Mehlhorn, H. (Ed.), Host Manipulations by Parasites and Viruses. Springer International Publishing, Cham, pp. 149-174.

2. Han Y , van Houte S, Drees GF, van Oers MM and Ros VID, 2015. Parasitic manipulation of host behavior: baculovirus SeMNPV EGT facilitates tree-top disease in Spodoptera exigua larvae by extending the time to death. Insects 6, 716-731.

3. Han Y , van Houte S, van Oers MM and Ros VID, 2017. Timely trigger of caterpillar zombie behaviour: Temporal requirements for light in baculovirus-induced tree-top disease. Parasitology 1-6.

4. Han Y , van Houte S, van Oers MM, Ros VID, 2018. Baculovirus PTP2 functions as a proapoptotic protein. Viruses $10,181$.

5. van Houte S, van Oers MM, $\underline{\text { Han Y }}$, Vlak JM, Ros VID, 2014. Baculovirus infection triggers a positive phototactic response in caterpillars to induce 'tree-top' disease. Biology Letters 10. $10,20140680$.

6. van Houte S, van Oers MM, $\underline{\text { Han Y }}$, Vlak JM, Ros VID, 2015. Baculovirus infection triggers a positive phototactic response in caterpillars to induce 'tree-top' disease: a response to Dobson et al. (2015). Biology Letters 11, 20150633.

7. Llopis-Gimenez A, Han Y, Kim Y, Ros VID, Herrero S, 2018. Identification and expression analysis of the Spodoptera exigua neuropeptidome under different physiological conditions. Submitted to Insect Molecular Biology, under review. 


\section{About the author}

Yue Han was born on July $31^{\text {th }}$, 1988 in Zhangjiakou, Hebei Province, China. After attending high school in Zhangjiakou, he moved to Nanjing, to start his BSc study in Aquaculture at Nanjing Agricultural University. After his BSc graduation, he performed his MSc study in Medical Biotechnology at Wageningen University. For his MSc minor thesis, he participated in the international Genetically Engineered Machine (iGEM) student competition in 2012. The team won the best oral presentation award at the European Jamboree and ranked among the top 16 (out of 191) teams at the Final Jamboree in Boston at the Massachusetts Institute of Technology (MIT). His MSc study was finalized by an MSc thesis on behavioural manipulation induced by baculoviruses at the Laboratory of Virology at Wageningen University. His MSc thesis was awarded with the Master Thesis Award from the Uyttenboogaart-Eliasen Stichting (UES). After his MSc graduation, Yue started his $\mathrm{PhD}$ at the Laboratory of Virology of Wageningen University \& Research, under supervision of Dr. Vera Ros and Prof. Dr Monique van Oers. For his dissertation, he continued the study of unravelling the molecular mechanisms of baculovirus-induced behavioural changes in insects. In August 2017 he received the Mauro Martignoni Award for the best $\mathrm{PhD}$ researcher from the Society of Invertebrate Pathology (SIP).

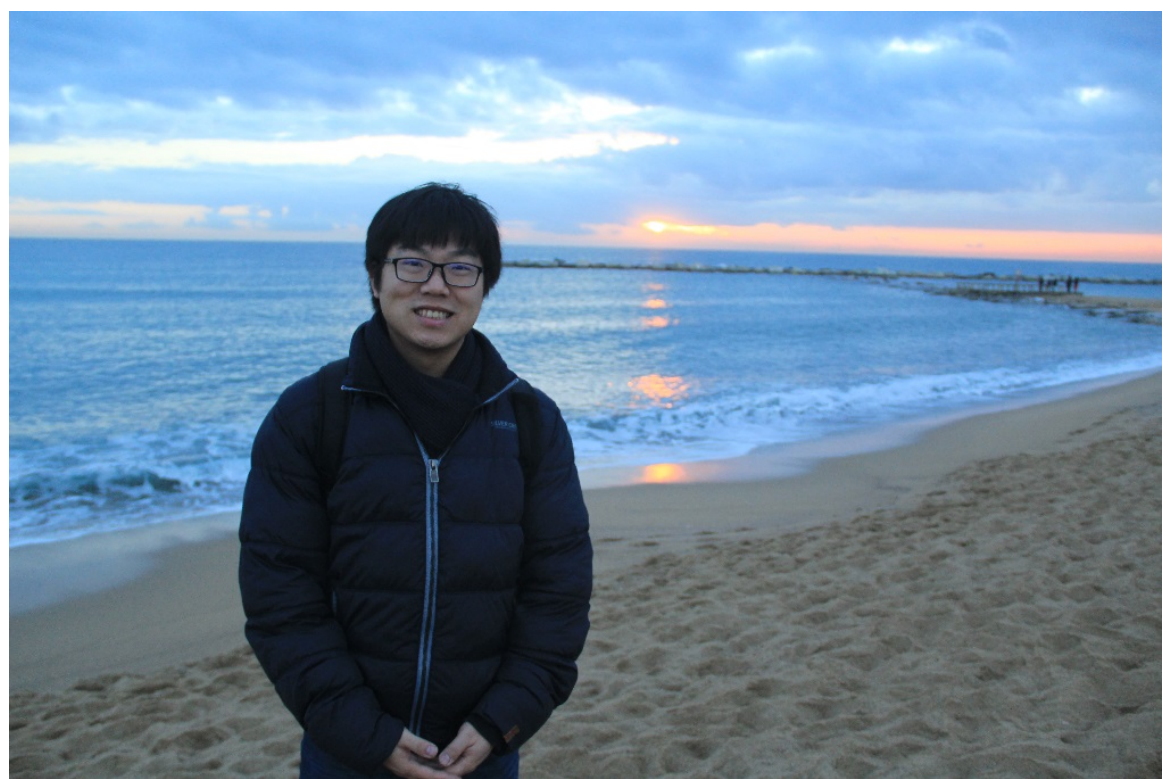




\section{Acknowledgements}

So, finally, it comes to an end. The acknowledgements turn out to be the most difficult part for me to write: not because I do not know what to say, but because I am not sure where to start. Of course it feels super good to finish everything (unfortunately not everything I planned), it also feels a bit empty when the journey comes to an end. Looking back, it feels super long, since I have been in Wageningen for seven years (fortunately it did not take me seven years to finish my $\mathrm{PhD}$ ). Meanwhile it also feels super short, since the start of my MSc just seems like yesterday. However, there is no doubt that the past seven years have been the most rewarding and fulfilling days in my life so far. I would like to take this opportunity to thank many amazing people I met during this journey.

Monique, I am not sure whether you still remember how we met for the first time. However, if I look back, I think the situation might have been quite demotivating for you. Since a student came to you to ask for a master thesis position (during the coffee break of the MSc course Immunotechnology) and the reason was that he got rejected by other groups. I still feel proud I asked anyway and I feel even more grateful that you provided me a MSc thesis position, which turned into a $\mathrm{PhD}$ position later on. This is just an example of the support you have given me since the very beginning. Your support allows me to do the things that I want to explore, and at the same time, gives me the space to become an independent thinker. Along the way, your great insights and active attitude guided the project in the right direction and we made some exciting discoveries. Also, whenever I wanted to discuss something, you always made time for me. The speed with which you reply to my emails and provided comments on my manuscripts is phenomenal: I often got an email back the same day and the manuscripts returned the second day after I'd sent it to you. This was extremely helpful in the final phase of thesis writing. It also feels special that I am the first $\mathrm{PhD}$ recruited by you when you became professor and chair of the group.

Vera, I could not have wished a better daily supervisor and co-promoter than you. Thank you for all your guidance, enthusiasm, new ideas and dedication to the project. All these made it easier for me to conduct the project. In general, I tend to get everything started ASAP and often neglect the overview and important details of the project. You work the opposite way. Gradually, I learned from you how to think ahead and how to control my pace to do a better job. I also highly appreciate the openness you have for me: we can discuss not only work-related problems but also personal issues. Not everything in this project went as we expected, however, 
discussions with you could always result in better ideas for the next experiments. I am also truly happy for you that you got the tenure track position at Virology and raised two lovely kids in the past few years.

I have been lucky enough to work closely with many colleagues and friends in the baculo-group throughout these years. First and foremost, Stineke, the supervisor of my master thesis. I cannot express how much you helped me just in a few sentences here. But the things I learned from you in the MS thesis period were extraordinarily helpful till the end of my $\mathrm{PhD}$. Also, your positive altitude was always encouraging for me during the $\mathrm{PhD}$ period when I felt down working with these evil caterpillars. I am the co-author of several papers on which you are the first-author and you are a co-author of several papers first-authored by me. Nothing can be better than this. I am glad that everything is going super well with you and Edze in Cornwall, all the best for the future. Jitte, when you started I already stopped in the lab. But your help was tremendous, especially for the chapter on caterpillar brains. I still feel very thankful for the nice pictures you made for Chapter 6 the day before I submitted the thesis. Good luck with your postdoc research and I am happy you are going to be a father soon. Just, thanks for many useful suggestions and feedback, especially when Vera and Monique both were not there. Sometimes the suggestion was just one sentence long, but sufficient to solve many problems. Jan, many thanks for the critical questions (especially during the weekly baculo-meetings) and for your help with the confocal and EM work. I also enjoyed the funny chats and nice BBQ's in your garden very much.

Leo, my Brazilian brother, I really miss the days we worked closely in the lab, helping each other and complaining about everything. And of course, the beer, the BBQ and the dishes you cooked (I am still using the recipe you taught me for making pasta and cassava). All the best for you and Brenda in Brazil and I am glad that you are going to be a father soon. Visiting you in Brazil is still on my to do list. I have served as paranymph for three colleagues so far, which bonded our friendship in a special way. Desrina, it still feels like yesterday that you came to Virology to put your worm samples into the freezer when you had just landed, but had no clue where to put them (we just had a rearrangement of the lab). It just happens naturally that I can talk about everything with you. The lovely chatting, the big laugh, the dinner time and especially the spiritual supports and encouragements from you means a lot to me. I wish all the best to you and Bambang, and a lot of success to your sons. Qiushi, you do not talk much, but every time I ask a question or a small favour, you always give me very detailed answers and help me out. The reunion in San Francisco was amazing. I wish you and Paul all the best there. 
Ali, the nice stories you told me about Pakistan were always interesting and eye-opening for me, which I highly appreciated. The time we spent in Mainz was also memorable. I am happy you finally finished you PhD after a long journey and wish you may continue the success in Pakistan. Bob, it was nice to have you as the companion guy in the lab and office, especially after 6 or 7 pm when everyone is gone. Your feedback on certain questions also helped me a lot. Your critical thinking and routine of hard working will guarantee high quality research. Good luck with finishing your PhD. I would also like to thank Els and Hanke for the consistent help in cell culturing and larvae rearing. Especially Els, who introduced me to everything the first day I came here. The bird watching excursion with you and Frank on October 31th, 2015 in Germany was also amazing (the night before that day Chunyue became my girlfriend, that is why I remember it so clearly). Special thanks also go to the Chinese visitors in the baculo-group: Jingfang and Fengqiao (my big baby), your company during the late nights and weekends made me feel less lonely. I also appreciate the time we spend outside the lab: the dinner, the fun conversation and trips to other cities which brought a lot of fun. Amaya, Mehtap, Irene Meki and Aydin, I have very good memories of you in the baculo-group, thanks for the fun time. Angel, thanks for sharing your knowledge on transcriptomics. We even managed to publish a paper together. I hope the time in Wageningen was also enjoyable for you as well.

At Virology, I felt at home throughout these years. Thanks for all the drinks, dinners, lab trips, fun parties and coffee breaks, which bring a lot of interesting topics and gossip to discuss, and laughter in return. Corinne, thanks for all the useful suggestions and help in the lab. Technically, I think I got most help from you. Dryas, Athos, Marcio, Jelke and Paulus, you guys started your $\mathrm{PhD}$ earlier than me and sparked me in one way or another on experimental design and how to handle the stress. Thank you for all the inspirations. Mia, when you were still around at Virology, it was always very cheerful to talk to you. Thanks for the fun time and I feel very happy for you and Jeroen. Giel, whenever I ask you a favour inside or outside the office, you are always willing to help. Help me to move to Javastraat, give me and my friend a drive to Enschede, help me with finding the right chemicals, antibodies and protocols for experiments. I also enjoyed very much the dinners, drinks and BBQ's at your place. Thank you for all of these. Min, André, Irene v. G. and Magda, thank you for the fun talks (especially when we make mean jokes, which usually were initiated by me, developed by you guys and ended with a big laugh) and company in the lab. Especially André, for teaching me to play guitar, sharing personal thoughts, giving very constructive feedbacks on my experimental design and assisting in developing those mean jokes. Sandra and Tessy, thank you for the nice talks in the office and 
I wish you two smart young scientists best of luck with your research. Thanks to Marleen and Dick L. for pointing me in the right direction when I needed help in the lab. When it comes to administrative issues, Marleen is always the first one to help me out. Also thanks to Richard and Gorben for the enlightening discussions about science and other things. Qiuhong, John and Fred, thank you for the nice time in the office.

The research conducted in this thesis also received great support from people from other departments or universities. Mark, thanks for teaching me how to prepare samples for the proteomics and for allowing me to use your lab bench whenever I needed it. Sjef, thanks for analysing all the proteomic samples so quickly and for replying to my emails instantly, this was of great help. Sabrina, thanks for all the help in analysing the transcriptomic data. Though these data eventually are not included in this thesis, I still learned a lot from the whole process. Thank you Hans for making some of the brain samples and most importantly for always giving constructive suggestions on the brain work. Thank you Elisabeth, Philip and Yannis for hosting me a week at your lab in Tours, France and for teaching me how to process transcriptomic data. It was a wonderful and fruitful week before Christmas in 2017. Last but not least, Angel, thanks for the nice work about transcriptomics. We even managed to publish a paper together. I hope the time in Wageningen is also enjoyable for you as well. You are very sharp in thinking and quick action will bring you success in science and beyond that.

I was lucky enough to work with the 12 best BSc and MSc students in the world. Without their contribution, the project could not have moved so smoothly. Thank you Berndjan, Lisa (Pennemann), Vera (Hesen), Gerben, Yijing, Amy, Kayleigh, Ioana, Daan, Pjotr, Iris and Justin, thank you for all your hard work and the funny moments in the lab. Special thanks to Hanneke, who made significant contributions to the brain mapping. I also learned a lot during the process of guiding you. Meanwhile, some of you have already started your own PhD study. I wish all of you the best of luck and of course a lot of success in the future.

I would like to thank my teammates from the iGEM competition, especially Kees, Hugo, Wouter and Marnix, from whom I learned a lot, in the lab and about designing a good project. The travelling to Munich and the trip to Boston for the final Jamboree were so much fun. I cannot imagine a better team than ours. I also want to thank people from the Wageningen Evolution and Ecology Seminars (WEES) series. Together, we invited quite some famous scientists from all over the world to give a seminar in Wageningen. It was exciting to talk with scientists from different fields. 
During the experiments, thousands of caterpillars were used (and killed). Maybe they also deserve a special thank you. Thanks for no matter how some of them were killed (starving, drowning, infecting, freezing, smashing, even microwaving), the survivors can still produce a lot of eggs for future experiments. I do feel very bad sometimes. Of course, the colony also collapsed, twice, which made me feel even worse.

Wageningen is a small town, life would be extremely boring without friends around. Corien, thanks for being my paranymph. I still remember you started your $\mathrm{PhD}$ on the day of the lab trip that was organized by me. I was talking to you and we got lost in the forest at a certain time point. I told you I knew the way back to Wageningen, which turned out to be not true. Well, that was only the first impression. Thanks for all the fun talks, suggestions and for not getting angry about the mean jokes. Best of luck with finishing your PhD. Yuxi, thanks for being my other paranymph. First and foremost, of course thank you for introducing me to Chunyue, which completely changed my life. 一起旅行, 聚餐, 打牌的时光, 总是感到枯燥日子里最好的 调剂。不知不觉, 七年的时间已经过去了, 很荣幸一路以来, 都有你的分享和帮助。

From my master to $\mathrm{PhD}$, I have been lucky to meet so many friends and share fun moments with them along the journey. If I listed all the names and fun things we did together, it would be another book much thicker than the thesis, so I have to keep it short here. 刘痘痘，王萌萌， 周点点, 从硕士开始, 最开心和最烦恼的事情一定都是第一时间找你们分享, 出谋划策。 在感慨时光飞逝的时候, 又庆幸在时光中我们越走越近。无论每次语音和视频, 都能 无拘无束的闲聊。每次回国, 时间再紧张也都一定会去找你们聚聚, 感谢你们一直都 是我最亲近的小伙伴。特别是老刘, 感谢的话反而不知道怎么说了, 真心的希望你在 喜欢的事情上越做越大。熊路, 总是很欣赏你乐观又坚强的态度, 你身上总能体现出 别人身上少有的责任感。感谢你在老刘他们走后, 又把我带到了另外的一个小团体里。 每次视频和回国, 也总能在深入探讨一些话题的时候获得很多新的思路。陈洋, 大哥, 大嫂, 每次大家一起吃饭或者出去玩, 总能有那么多好玩的话题或者事情来讨论。更 感谢你们在很多重要的事情上为我出谋划策。大哥和大嫂的车不但成了方便我和春月 出行的交通工具, 更是无限的延后了我学驾照的时间。大家未来一年都要陆续毕业了, 一起加油。还有王荔（不是这个力）, 冯媛, 张杰, 静宜, 张岗, 郝叔叔, 郭霄汉, 凡哥, 大师, 小西, 郝老师, 真爱（何源），丽娅姐，刘珏，邱总，眭杰，眭一星， 朱峰, 小岳岳, 亦如, 熊弟弟, 感谢大家在不同的阶段的陪伴, 那都是在异国他乡最 
美好的回忆。Lucrecia, thanks for the nice talks and fun times in and outside the lab. Wim, I still miss all the beer time and chat after work. Glad you daughter is doing well now.

还有阿楠, 能有一个无话不谈的发小是我最庆幸的事情。每当我有疑问, 困惑, 你总 是最大限度的帮助我。每次回国, 必做的事情就是好好和你长聊一次。这些年来, 看 你一步步在自己喜欢的工作上做得风声水起，更是为你感到开心。

爸爸, 妈妈, 爷爷, 奶奶, 这些年在荷兰, 回家的时间真的少得可怜。每次回国, 其 实在家里待的时间也很少, 都被我用来东奔西跑了。成长中我觉得最庆幸的事情就是 老爸老妈给我了最大的自主选择的权利, 最大限度的支持我的选择。事情做的好, 你 们会鼓励, 希望我继续保持, 事情没做好, 也从没说我什么。唯一一直叮嘱我的就是 注意身体。爷爷奶奶更是我的软肋, 每次离家前都会哭成泪人。今后的日子, 一定会 多陪在你们身边, 我爱你们。

小花花, 都说陪伴是最长情的告白, 这可能是现在最能表达我感受的一句话。感谢这 些年在生活上对我无微不至的照顾和对我选择无限的支持。有条不紊的你是对着急忙 慌的我最好的补充。我无限的期待我们接下来的生活, 和你一起去旅行, 做很多精彩 的事情, 包括成立我们自己的家庭, 然后一起慢慢变老！爱你！

Chunyue, I think only "company is the longest confession of love" can express my feeling now. Thank you for the meticulous care and unconditional support throughout these years. I look forward to the next stage of our life!! Most importantly, we can travel to other countries and do many other wonderful things together, including having our own family and getting old together. Love you!!

Yue Han/韩越 July $6^{\text {th }}, 2018$ Wageningen 
PE\&RC Training and Education Statement

With the training and education activities listed below the $\mathrm{PhD}$ candidate has complied with the requirements set by the C.T. de Wit Graduate School for Production Ecology and Resource Conservation (PE\&RC) which comprises of a minimum total of 32 ECTS $(=22$ weeks of activities)

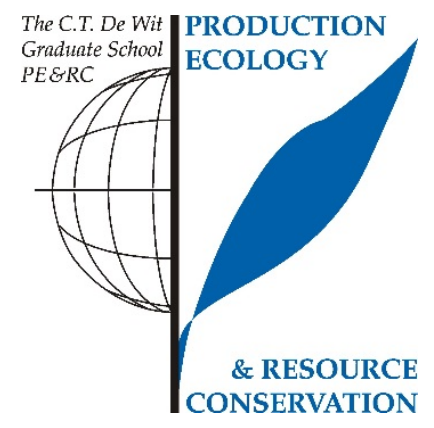

\section{Review of literature (6 ECTS)}

- Virus-induced behavioural changes in insects (2015)

\section{Writing of project proposal (4.5 ECTS)}

- Deciphering the mechanisms underlying baculovirus-induced behavioral changes in insects: using proteomics and transcriptomics as tools

\section{Post-graduate courses (6.2 ECTS)}

- The power of RNA-seq; Wageningen University (2013)

- Introduction to R for statistical analysis; Wageningen University (2014)

- $\quad 5^{\text {th }}$ International advanced course: proteomics; Wageningen University (2015)

- Bioinformatics: a user's approach; Wageningen University (2015)

- Survival analysis; Wageningen University (2016)

- Genome-free RNA-seq assembly and transcriptome analysis leveraging trinity; Physilia courses, Berlin (2017)

\section{Laboratory training and working visits (1.5 ECTS)}

- $\quad$ RNA-seq data analysis; Institut de Recherche sur la Biologie de Insecte (IRBI), Tours, France (2016)

\section{Invited review of (unpublished) journal manuscript (2 ECTS)}

- Biotechnology Progress: baculovirus expression system (2016)

- Plos Biology: behavioural manipulation (2017)

\section{Deficiency, refresh, brush-up courses (1.5 ECTS)}

- Basic statistics; Wageningen University

\section{Competence strengthening / skills courses (5.9 ECTS)}

- Competence assessment; Wageningen University (2013)

- Scientific writing; Wageningen University (2014)

- Entrepreneurship in and outside of science; Wageningen University (2014) 
- Scientific publishing; Wageningen University (2015)

- Pitch perfect; Wageningen University (2016)

- Career orientation; Wageningen University (2017)

PE\&RC Annual meetings, seminars and the PE\&RC weekend (3.3 ECTS)

- PE\&RC weekend, first year (2013)

- PE\&RC Day $(2013,2015,2017)$

- $\quad$ PE\&RC weekend, mid term (2015)

- PE\&RC weekend, last year (2017)

Discussion groups / local seminars / other scientific meetings (8.5 ECTS)

- Wageningen Evolution and Ecology Seminars; organization committee 2014-2017 (20132017)

- $\quad 2^{\text {nd }}$ Wageningen $\mathrm{PhD}$ symposium (2015)

- Insect-plant interactions lunch meeting; Laboratory of Entomology (2016-2017)

\section{International symposia, workshops and conferences (21.8 ECTS)}

- Seventh international symposium on Molecular Insect Science (2013)

- Annual meeting of the Society for Invertebrate Pathology (2013-2017)

- Annual Dutch entomology day (2013-2017)

- Dutch annual virology symposium $(2014,2015,2017)$

- Revolutionizing next-generation sequencing: tools and technologies (2015)

\section{Lecturing / Supervision of practicals / tutorials (6.9 ECTS)}

- Molecular virology (2014-2015)

- Immunotechology (2014-2017)

- Molecular aspects of biointeractions (2015)

\section{Supervision of MSc students}

- Baculovirus induces behavioural changes via invading the host central nervous system Yijing Wang

- Exploring the role of SeMNPV ptp2 in apoptosis, and substrates analysis of SePTP2 - Ioana Nicorescu

- Characterizing long transcripts and miRNA from the SeMNPV egt region- Pjotr Middendorf 
Printing: Ridderprint $B V \mid \underline{w w w . r i d d e r p r i n t . n l}$

Cover design: Sofie Bernhagen 